\title{
Towards numerical
}

simulation of components

of thermoacoustic devices

with commercial CFD software

Implementation of impedance boundary conditions and application to four different studies 



\section{Towards numerical simulation of components of thermoacoustic devices with commercial CFD software}

Implementation of impedance boundary conditions and application to four different studies

Simon Bühler 
Samenstelling van de promotiecommissie:

Voorzitter:

Prof. dr. G.P.M.R. Dewulf Universiteit Twente

Promotor:

Prof. dr. ir. T.H. van der Meer Universiteit Twente

Leden:

Prof. dr. ir. H.W.M. Hoeijmakers

Prof. dr. ir. A. Hirschberg

Universiteit Twente

Universiteit Twente

Prof. dr. ir. B.J. Boersma

Technische Universiteit Delft

Dr. Ph. Blanc- Benon

Dr. ir. J.C.H. Zeegers

LMFA CNRS - École Centrale de Lyon

D.A. Wilcox

Technische Universiteit Eindhoven

Chart Industries, Inc.

\section{UNIVERSITY OF TWENTE.}

Faculty of Engineering Technology

Laboratory of Thermal Engineering

The research in this thesis was financially supported by Agentschap NL as part of the EOS-KTO research program under Project No.KTOT03009.

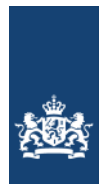

Copyright (C) 2015 Simon Bühler

Cover image: Enclosure with isothermal walls subject to a standing wave $(\lambda / 2)$ at the moment of zero crossing of the pressure perturbation.

ISBN 978-90-365-3909-8

DOI $\quad 10.3990 / 1.9789036539098$ 


\title{
TOWARDS NUMERICAL SIMULATION OF COMPONENTS OF THERMOACOUSTIC DEVICES WITH COMMERCIAL CFD SOFTWARE
}

\author{
Implementation of impedance boundary conditions \\ and application to four different studies
}

\section{PROEFSCHRIFT}

\author{
ter verkrijging van
}

de graad van doctor aan de Universiteit Twente,

op gezag van de rector magnificus,

prof. dr. H. Brinksma

volgens besluit van het College voor Promoties

in het openbaar te verdedigen

op vrijdag 10 juli 2015 om 16:45 uur

door

Simon Bühler

geboren op 26 augustus 1986

te Herrenberg, Duitsland 
Dit proefschrift is goedgekeurd door:

Prof. dr. ir. T.H. van der Meer

Copyright (C) 2015 Simon Bühler

ISBN 978-90-365-3909-8 


\section{Summary}

Thermoacoustic engines promise to be a cost effective and reliable alternative to traditional Stirling engines, as the function of the piston is fulfilled by an acoustic wave. For the design and development of thermoacoustic devices, the one-dimensional thermoacoustic equations are commonly used. However, to further improve the performance of these devices a better understanding of the flow field and the acoustic losses inside of thermoacoustic components is required. To gain further insight, commercial Computational Fluid Dynamics (CFD) software is used in this thesis, as CFD allows revealing the entire flow field with all its physical quantities in the respective component of the thermoacoustic device. Reducing the numerical study to individual components of thermoacoustic devices is only possible with dedicated acoustic boundary conditions. As these boundary conditions are not yet readily available in commercial CFD packages, their implementation into ANSYS Fluent is included within the scope of this work. The boundary conditions are validated successfully in one- and two-dimensional cases against analytical solutions from the low-reduced frequency approximation. Furthermore, the analytical solutions are used in order to derive the optimal numerical parameters for thermoacoustic simulations and to give general rules of thumb for the spatial and time discretization.

In a second step these parameters are applied to the simulations of four different thermoacoustic cases in order to show that CFD can lead to a better understanding of phenomena that are not incorporated in the one-dimensional thermoacoustic equations.

The first investigated component is the thermal buffer tube. Its aim is to provide thermal insulation between the hot heat exchanger and the secondary ambient heat exchanger while transmitting the acoustic power out of the hot zone. However, due to the interaction of the acoustic wave with the temperature gradient, a two-dimensional steady mass flux called acoustic streaming occurs, which leads to undesired thermal losses. Using the implemented ideal heat exchanger boundary condition, the two-dimensional streaming field inside the thermal buffer tube is revealed and the influence of the wall properties on the streaming pattern is estimated. The temperature field resulting from the different streaming patterns as well as the repercussions on the acoustic properties are shown.

The second investigated component is the U-bend that feeds back the acoustic wave in a traveling wave device. In the scope of reducing the size of the thermoacoustic devices, the bend becomes increasingly sharp, introducing additional losses and reflection as well as a velocity component in the cross-direction, which is expected to influence nearby components such as heat exchangers. The influence of the geometric parameters of the 
bend on the flow field are investigated in this thesis. The deviations from the analytical solution are revealed and for even sharper bends the onset of vortex generation is given. In general, this study shows the strength of numerical CFD simulations in thermoacoustics, as a large geometric parameter space could be investigated, leading to an in-depth understanding of the underlying flow phenomena.

The subsequent study in this thesis makes the link between the one-dimensional thermoacoustic equations and the full time domain CFD, as it shows how the accuracy of the results from the one-dimensional equations can be increased when data from CFD is used. In this work the thermoacoustic functions, which incorporate the three-dimensional effects in the one-dimensional thermoacoustic equations, are calculated from CFD for a reduced model of a stacked screen regenerator, leading to more realistic values of the thermoacoustic functions. It is shown that the arrangement of the screens has an effect on the heat transfer inside the regenerator, while the viscous effects stay the same. This study shows that not only large components like bends and the thermal buffer tube can be successfully simulated with CFD, but so can small scale geometries like the ones inside the regenerator.

In the last thermoacoustic study within this work, the entrance effects in a stacked screen regenerator are investigated for different geometric variations. The mean temperature profile due to the non-linear entrance effects and the heat pumped at the end of the stacked screen regenerator are calculated. Furthermore, a one-dimensional time dependent heat equation is used in order to predict the changes in mean temperature. In this onedimensional time dependent heat transfer equation the thermal thermoacoustic function is used in order to estimate the heat transfer coefficient between the regenerator and the fluid. The results compare well with the CFD results.

It can be concluded from the four studies conducted within this thesis that the simulation of components of thermoacoustic devices with commercial CFD is possible and that they will contribute to a better understanding of the flow phenomena inside of the respective components. This work paves the way towards the in-depth investigation of other components within the field of thermoacoustics using CFD. 


\section{Samenvatting}

Thermoakoestische motoren kunnen in potentie uitgroeien tot een kostenefficiënt en betrouwbaar alternatief voor de traditionele Stirlingmotoren, aangezien de functie van de zuiger wordt vervuld door een akoestische golf. Voor het ontwerp en de ontwikkeling van thermoakoestische apparaten, wordt doorgaans gebruikgemaakt van eendimensionale thermoakoestische vergelijkingen. Om de prestaties van deze apparaten verder te verbeteren is echter een beter begrip vereist van het stromingsveld en de akoestische verliezen binnen de thermoakoestische onderdelen. Om tot een beter inzicht te komen wordt voor dit onderzoek gebruikgemaakt van commerciële numerieke stromingsleer (Computational Fluid Dynamics of CFD). Hiermee kan het gehele stromingsveld met alle grootheiden in de betreffende component van het thermoakoestische apparaat in kaart worden gebracht. Het terugbrengen van de numerieke studie tot afzonderlijke componenten van thermoakoestische apparaten is alleen mogelijk door gebruik te maken van akoestische randvoorwaarden. Omdat deze randvoorwaarden niet standard beschikbaar zijn in commerciele CFD-pakketten, maakte de implementatie hiervan in ANSYS Fluent deel uit van dit onderzoek. De randvoorwaarden zijn met succes in één- en tweedimensionale gevallen gevalideerd tegen analytische oplossingen van het zogenaamde Low Reduced Frequency model. Daarnaast wordt er gebruikgemaakt van deze analytische oplossingen om te komen tot optimale numerieke parameters voor thermoakoestische simulaties en om te zorgen voor algemene vuistregels ten aanzien van de ruimte- en tijdsdiscretisatie.

Tijdens een volgende stap zijn deze parameters toegepast op de simulaties van vier verschillende thermoakoestische situaties. De bedoeling is aan te tonen dat CFD kan leiden tot een beter inzicht in verschijnselen die niet worden beschreven door de eendimensionale thermoakoestische vergelijkingen.

De eerste onderzochte component is de zogenaamde thermal buffer tube. Het doel hiervan is te zorgen voor thermische isolatie tussen de hete warmtewisselaar en de secundaire warmtewisselaar op omgevingstemperatuur, tijdens de overdracht van het akoestisch vermogen uit de warme zone. Als gevolg van de wisselwerking tussen de akoestische golf en de temperatuurgradiënt, ontstaat er een tweedimensionale continue massastroom die we acoustic streaming noemen en die leidt tot ongewenste warmteverliezen. Met behulp van de geïmplementeerde randvoorwaarde voor de warmtewisselaar wordt het tweedimensionale stromingsveld binnen de thermal buffer tube opgelost. Zo kan een schatting worden gemaakt van de invloed van de wandeigenschappen op het stromingspatroon. Het temperatuurveld als gevolg van de verschillende stromingspatronen evenals de effecten daarvan op de akoestische eigenschappen worden in beeld gebracht. 
De tweede onderzochte component is de U-bocht die de akoestische golf geleidt. Om thermoakoestische apparaten compacter te maken, moet de bocht steeds scherper worden. Hierdoor ontstaan extra verliezen en reflectie, evenals een snelheidscomponent in de radiale richting, die naar verwachting de nabije componenten zoals de warmtewisselaars beïnvloedt. In dit proefschrift wordt de invloed van de geometrische parameters van de bocht op het stromingsveld onderzocht. De afwijkingen van de analytische oplossing worden beschreven en voor nog scherpere bochten wordt het ontstaan van de gegenereerde vortex gegeven. Over het algemeen laat dit onderzoek de kracht zien van numerieke CFD-simulaties in de thermoakoestiek, aangezien een grote geometrische parameterruimte kan worden onderzocht. Dit resulteert in een gedetailleerd inzicht in de achterliggende stromingsverschijnselen.

In het daaropvolgende onderzoek in dit proefschrift wordt een verband gelegd tussen de eendimensionale thermoakoestische vergelijkingen en CFD. Dit geeft aan hoe de precisie van de resultaten van de eendimensionale vergelijkingen kan worden vergroot door gebruik te maken van CFD-gegevens. Hierbij worden de thermoakoestische functies, waarbij driedimensionale effecten in de eendimensionale thermoakoestische vergelijkingen zijn opgenomen, berekend op basis van CFD voor een gereduceerd model van een stacked-screen regenerator. Dit resulteert in realistischer waarden voor de thermoakoestische functies. Aangetoond wordt dat de rangschikking van de screens een effect heeft op de warmteoverdracht in de regenerator, terwijl de viskeuze effecten gelijk blijven. Uit het onderzoek blijkt dat niet alleen grote componenten zoals bochten en de thermal buffer tube met succes met CFD kunnen worden gesimuleerd, maar ook kleinschalige geometrische aspecten zoals die aanwezig zijn in de regenerator.

In het laatste thermoakoestische onderzoek van dit project, zijn de intree-effecten in een stacked-screen regenerator onderzocht voor verschillende geometrische variaties. Het tijdgemiddelde temperatuurprofiel als gevolg van de niet-lineaire effecten bij intrede en de warmte die wordt gepompt aan het einde van de stacked-screen regenerator zijn beschreven. Daarnaast wordt gebruikgemaakt van een eendimensionale tijdsafhankelijke warmtevergelijking om de veranderingen in de gemiddelde temperatuur te voorspellen. In deze vergelijking wordt de thermische thermoakoestische functie gebruikt om de warmteoverdrachtscoëfficiënt tussen de regenerator en het gas te schatten. De resultaten daarvan laten zich goed vergelijken met de CFD-resultaten.

Uit de vier in het kader van dit proefschrift verrichte onderzoeken kan worden geconcludeerd dat het simuleren van componenten van thermoakoestische apparaten met commerciële CFD mogelijk is en dat dit kan bijdragen aan een beter begrip van de stromingsverschijnselen binnen de desbetreffende componenten. Dit onderzoek maakt de weg vrij voor verder onderzoek naar overige thermoakoestische componenten met behulp van CFD. 


\section{Contents}

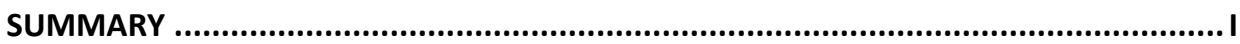

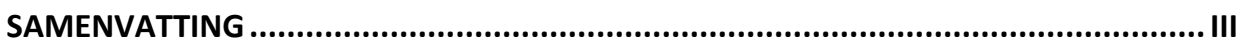

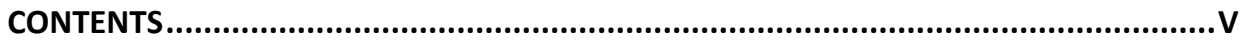

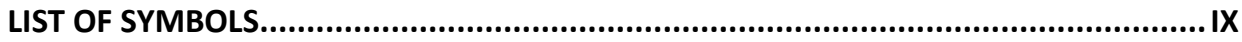

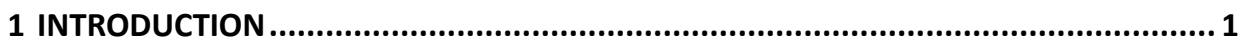

1.1 THERMOACOUSTIC DEVICES............................................................................... 2

1.2 CFD MODELING IN THERMOACOUSTICS ............................................................. 3

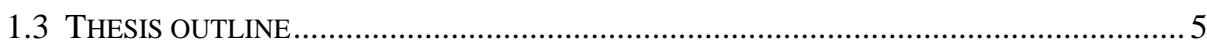

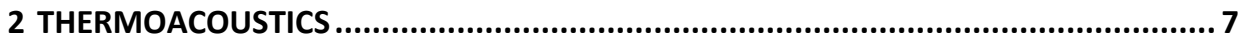

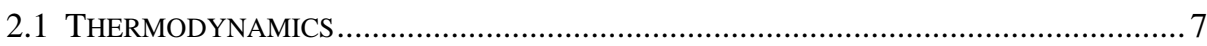

2.1.1 Laws of thermodynamics.......................................................................... 7

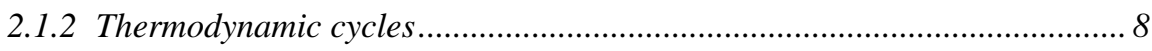

2.1.2.1 Standing wave phasing ......................................................... 8

2.1.2.2 Traveling wave phasing ......................................................... 11

2.1.2.3 Bucket-brigade effect.......................................................... 12

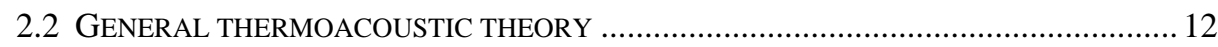

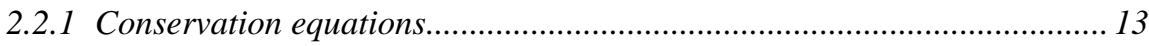

2.2.2 Linearization and Fourier transformation ................................................ 14

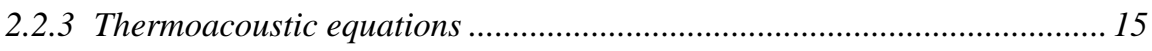

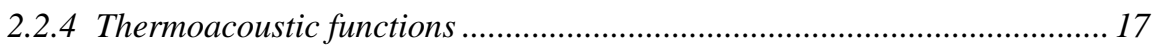

2.2.4.1 Existing analytical solutions ................................................. 18

2.2.4.2 Numerical integration ......................................................... 18

2.2.4.3 Experimental estimation of the thermoacoustic functions ............ 19

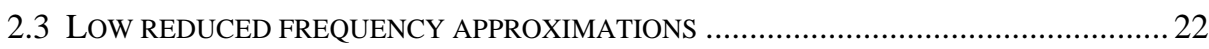

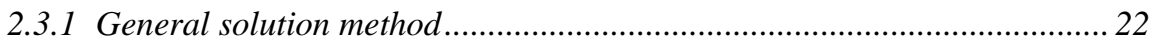

2.3.2 Straight tube with circular cross-section .................................................. 24

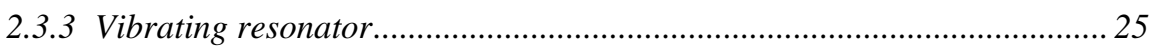

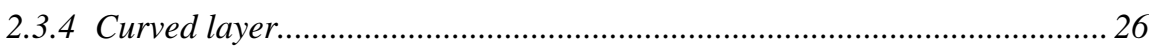

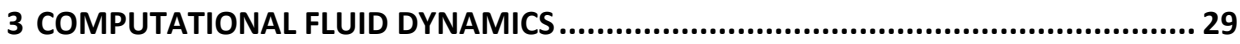

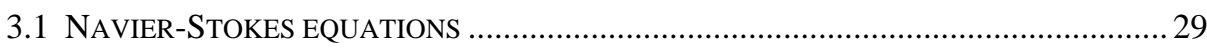

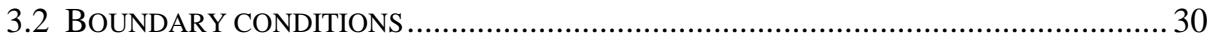

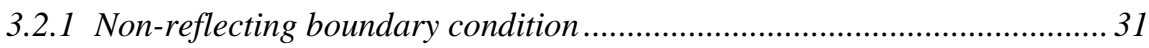

3.2.1.1 One-dimensional wave propagation ......................................... 32 
3.2.1.2 Wave propagation in a pipe.

3.2.1.3 Extension of the non-reflecting boundary condition for nonideal wave propagation

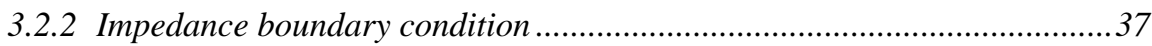

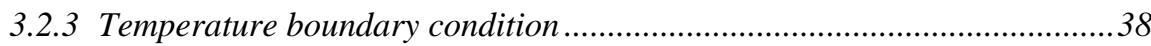

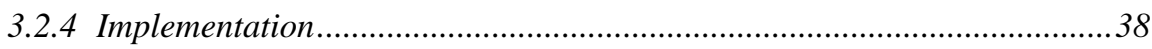

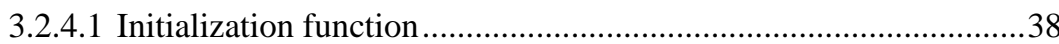

3.2.4.2 Function to calculate the new pressure values ..............................38

3.2.4.3 Function to apply the boundary condition....................................40

3.3 LIMITATION OF THERMOACOUSTIC CFD SIMULATIONS ........................................40

3.3.1 Spatial scales......................................................................................... 40

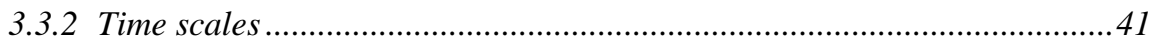

3.3.3 Coupling between time and spatial discretization.....................................41

4 VALIDATION FOR THERMOACOUSTIC SIMULATIONS ....................................... 43

4.1 ACOUSTIC BOUNDARY LAYER DISCRETIZATION .................................................43

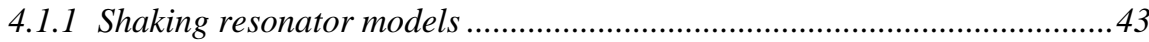

4.1.1.1 Low reduced frequency approximation.........................................44

4.1.1.2 Numerical model..............................................................................44

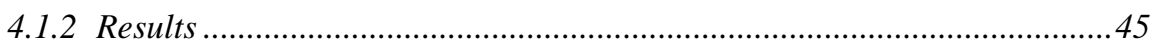

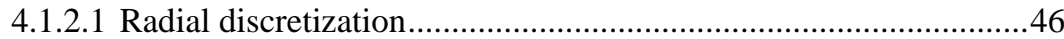

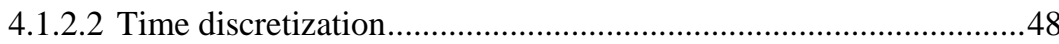

4.1.2.3 Axial discretization ....................................................................48

4.2 ONE-DIMENSIONAL NON-REFLECTING BOUNDARY CONDITION - WAVE

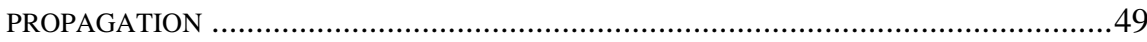

4.2.1 One-dimensional traveling wave model ......................................................50

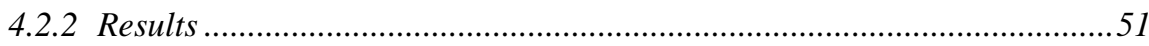

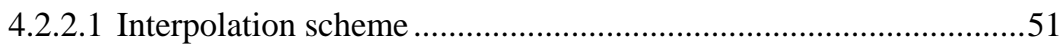

4.2.2.2 Spatial discretization schemes................................................54

4.2.2.3 Influence of the wave propagation ...........................................55

4.3 ONE-DIMENSIONAL IMPEDANCE BOUNDARY CONDITION ....................................57

4.3.1 One-dimensional standing wave resonator model ....................................57

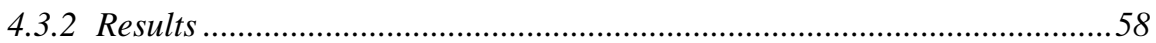

4.4 TWO-DIMENSIONAL IMPEDANCE BOUNDARY CONDITION ........................................59

4.4.1 Two-dimensional wave propagation model.................................................6 60

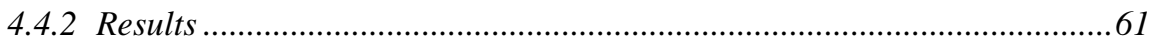

4.5 IDEAL HEAT EXCHANGER BOUNDARY CONDITION .................................................64

4.5.1 Ideal heat exchanger models ....................................................................64

4.5.1.1 Analytical solution ................................................................64

4.5.1.2 CFD model......................................................................65 


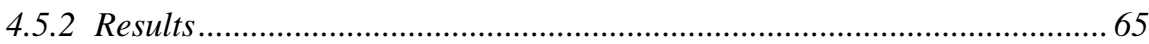

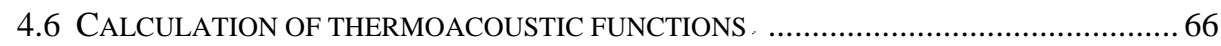

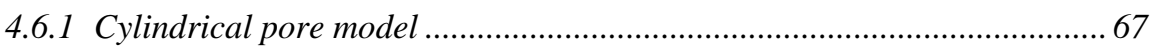

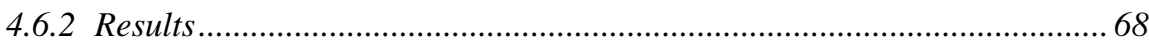

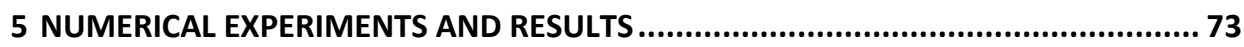

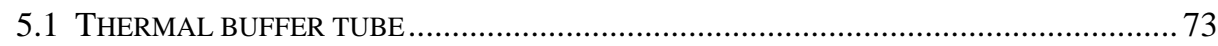

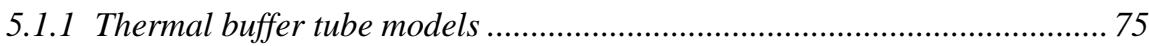

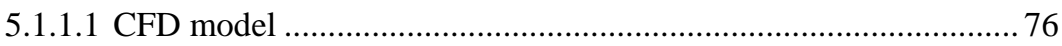

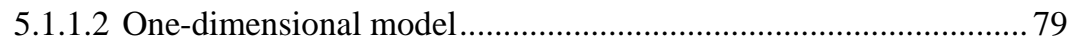

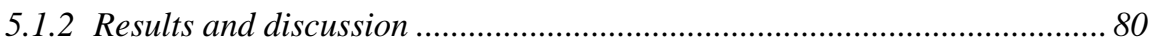

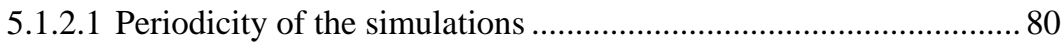

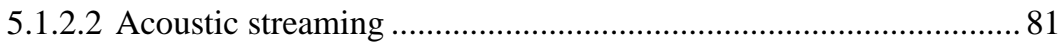

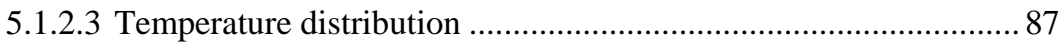

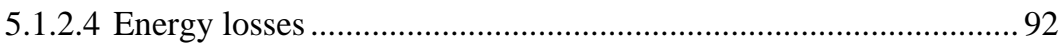

5.1.3 Conclusion and recommendations ...................................................... 97

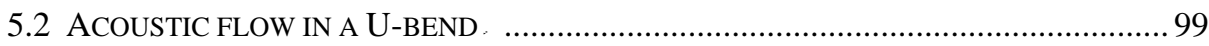

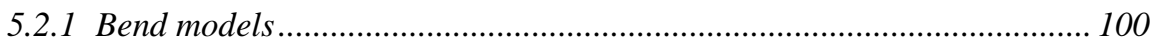

5.2.1.1 Curved layer CFD model....................................................... 101

5.2.1.2 Low reduced frequency model ............................................... 102

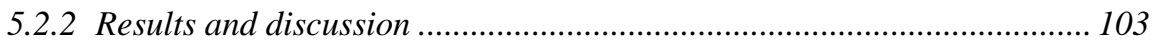

5.2.2.1 Curvature induced reflections ................................................ 105

5.2.2.2 Transitions from straight to bent............................................. 107

5.2.2.3 Limits of the analytical solution ............................................. 111

5.2.2.4 Flow field for extreme curvatures............................................ 114

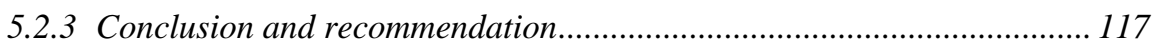

5.3 CAlCUlation OF THERMOACOUSTIC FUnCTIONS WiTH CFD ............................. 119

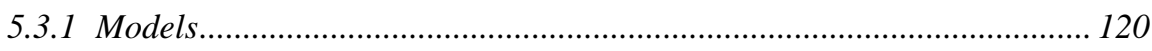

5.3.1.1 CFD Model .......................................................................... 121

5.3.1.2 One-dimensional model........................................................ 122

5.3.2 Results and discussion ..................................................................... 124

5.3.2.1 Influence of the number of screens........................................... 129

5.3.2.2 Influence of the cylinder arrangements.................................... 131

5.3.2.3 Influence of the drive ratio .................................................. 132

5.3.3 Conclusion and recommendations ...................................................... 133

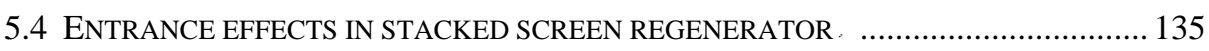

5.4.1 Entrance effect models ..................................................................... 137

5.4.1.1 CFD model .......................................................................... 137

5.4.1.2 One-dimensional model............................................................ 139

5.4.2 Results and discussion ...................................................................... 140 
5.4.2.1 Influence of the regenerator length 145

5.4.2.2 Influence of different openings of the meshed screen 147

5.4.2.3 Influence of the wave phasing. 150

5.4.3 Conclusion and recommendations. 153

6 CONCLUDING REMARKS 155

7 BIBLIOGRAPHY . 159

8 APPENDICES 169

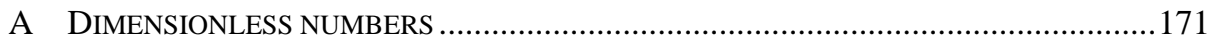

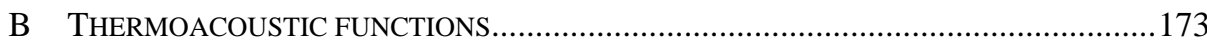

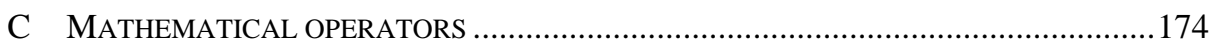

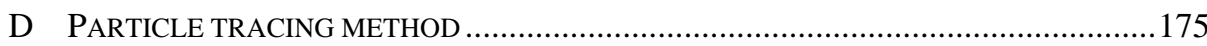

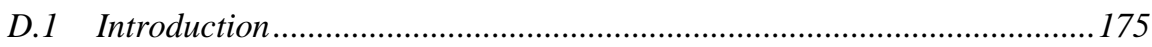

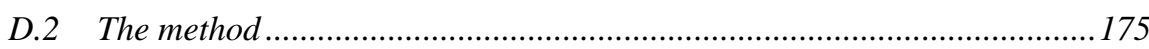

D.3 Interpolation..................................................................................... 177

D.4 Correction .................................................................................... 178

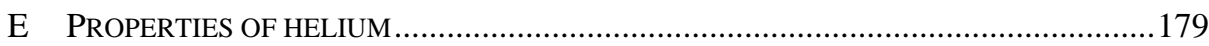

E.1 Temperature dependent properties......................................................... 180

F SHAKING RESONATOR - CHANGE IN COORDINATE SYSTEM................................... 181

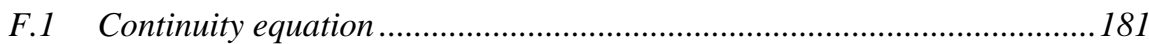

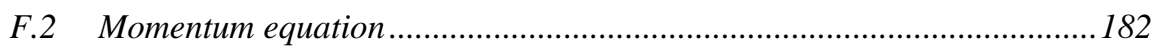

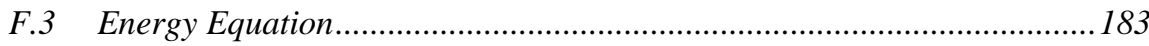

G POINT DEFINITION FOR SHAKING RESONATOR .................................................. 184

H SOLVING THE HEAT EQUATION WITH MATLAB .............................................. 185

I INFLUENCE OF ERRORS ON CALCULATION OF THERMOACOUSTIC FUNCTIONS .......187

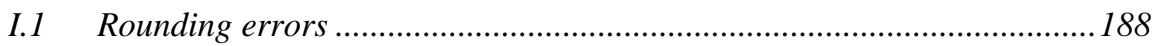

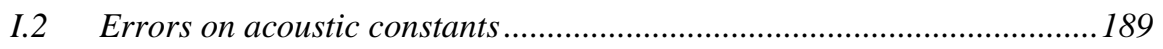

J SCALING OF THERMAL THERMOACOUSTIC FUNCTIONS ...................................... 191

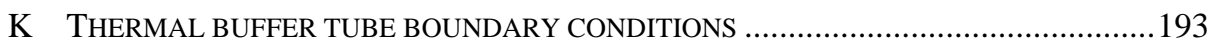

K.1 Non-reflecting boundary conditions....................................................... 193

K.2 Reflecting boundary condition ............................................................ 194

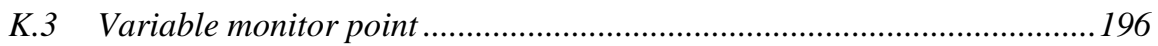

L THERMAL BUFFER TUBE MESH REFINEMENT ..................................................... 198

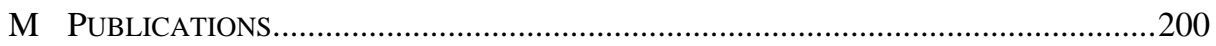

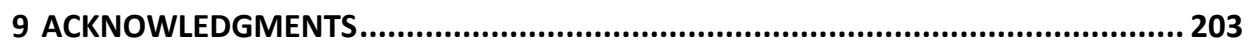




\section{List of symbols}

\section{Latin letters}

\begin{tabular}{|c|c|c|}
\hline Symbol & Unit & Signification \\
\hline$A$ & $\mathrm{~m}^{2}$ & Area \\
\hline$c$ & $\mathrm{~m}^{2} / \mathrm{Pa}$ & Compliance per unit length \\
\hline$c$ & $\mathrm{~m} / \mathrm{s}$ & Speed of sound \\
\hline$c_{0}$ & $\mathrm{~m} / \mathrm{s}$ & Undisturbed speed of sound \\
\hline$c_{p}$ & $\mathrm{~J} /(\mathrm{kg} \mathrm{K})$ & Specific heat capacity at constant pressure \\
\hline$c_{v}$ & $\mathrm{~J} /(\mathrm{kg} \mathrm{K})$ & Specific heat capacity at constant volume \\
\hline COP & - & Coefficient of performance \\
\hline$D$ & $\mathrm{~m}$ & Diameter \\
\hline$D r$ & - & Drive ratio \\
\hline$e$ & $\mathrm{~J} / \mathrm{kg}$ & Specific energy \\
\hline$E$ & $\mathrm{~J}$ & Energy \\
\hline$\dot{E}$ & $\mathrm{~W}$ & Power \\
\hline$\vec{e}_{i}$ & - & Unit vector in $i$-direction \\
\hline$\vec{F}$ & $\mathrm{~N} / \mathrm{m}^{3}$ & $\begin{array}{l}\text { Body forces in momentum conservation } \\
\text { equation }\end{array}$ \\
\hline Fo & - & Fourier number \\
\hline$f$ & $\mathrm{~Pa}$ & Forward traveling wave \\
\hline$f$ & $\mathrm{~Hz}$ & Frequency \\
\hline$f_{\kappa}$ & - & Thermal thermoacoustic function \\
\hline$f_{v}$ & - & Viscous thermoacoustic function \\
\hline$g$ & $\mathrm{~Pa}$ & Backwards traveling wave \\
\hline$g$ & $\mathrm{~m} / \mathrm{s}^{2}$ & Acceleration of gravity \\
\hline$h$ & $\mathrm{~m}$ & Half spacing \\
\hline$h$ & $\mathrm{~J} / \mathrm{kg}$ & Specific enthalpy \\
\hline$h_{\kappa}$ & - & Heat diffusion function \\
\hline$h_{v}$ & - & Momentum diffusion function \\
\hline
\end{tabular}




\begin{tabular}{|c|c|c|}
\hline Symbol & Unit & Signification \\
\hline$I$ & - & Identity matrix \\
\hline$i$ & - & $\sqrt{-1}$ \\
\hline$J$ & - & Bessel function \\
\hline K & $1 / \mathrm{s}$ & Heat transfer coefficient \\
\hline$K C$ & - & Keulegan-Carpenter \\
\hline$k$ & - & Reduced frequency \\
\hline$k$ & $\mathrm{~W} /(\mathrm{m} \mathrm{K})$ & Thermal conductivity \\
\hline$k$ & $1 / \mathrm{m}$ & Wave number \\
\hline$L$ & $\mathrm{~m}$ & Length \\
\hline$L c$ & - & Lautrec number \\
\hline$M$ & - & Mach number \\
\hline$m$ & $\mathrm{~kg}$ & Mass \\
\hline$n$ & - & Number of elements \\
\hline$n$ & - & Kind of polytropic constant \\
\hline $\operatorname{Pr}$ & - & Prandtl number \\
\hline$p$ & $\mathrm{~Pa}$ & Pressure \\
\hline$Q$ & $\mathrm{~J}$ & Heat \\
\hline$\dot{Q}$ & $\mathrm{~W}$ & Heat transfer rate \\
\hline$q$ & - & Growing factor \\
\hline$q$ & $\mathrm{~J} / \mathrm{kg}$ & Specific heat \\
\hline$R$ & $\mathrm{~m}$ & Radius \\
\hline$R$ & - & Reflection coefficient \\
\hline$R_{S}$ & $\mathrm{~J} /(\mathrm{kg} \mathrm{K})$ & Specific gas constant \\
\hline$R e$ & - & Reynolds number \\
\hline$r$ & $\mathrm{~m}$ & Radial coordinate \\
\hline$r_{h}$ & $\mathrm{~m}$ & Hydraulic radius \\
\hline$S$ & - & Safety factor \\
\hline$S_{h}$ & $\mathrm{~W} / \mathrm{m}^{3}$ & $\begin{array}{l}\text { Volumetric heat source in energy conserva- } \\
\text { tion equation }\end{array}$ \\
\hline$S_{m}$ & $\mathrm{~kg} /\left(\mathrm{m}^{3} \mathrm{~s}\right)$ & $\begin{array}{l}\text { Volumetric mass source in mass conserva- } \\
\text { tion equation }\end{array}$ \\
\hline
\end{tabular}




\begin{tabular}{|c|c|c|}
\hline Symbol & Unit & Signification \\
\hline$s$ & - & Shear wave number \\
\hline$s_{t}$ & - & Thermal shear wave number \\
\hline St & - & Strouhal \\
\hline$T$ & $\mathrm{~s}$ & Period \\
\hline$T$ & $\mathrm{~K}$ & Temperature \\
\hline$t$ & $\mathrm{~s}$ & Time \\
\hline$U$ & $\mathrm{~m}^{3} / \mathrm{s}$ & Volume flow rate \\
\hline$u$ & $\mathrm{~J} / \mathrm{kg}$ & Specific internal energy \\
\hline$u$ & $\mathrm{~m} / \mathrm{s}$ & $\mathrm{x}$ component of velocity \\
\hline$\vec{v}$ & $\mathrm{~m} / \mathrm{s}$ & Vector velocity \\
\hline$v$ & $\mathrm{~m}^{3} / \mathrm{kg}$ & Specific volume \\
\hline$v$ & $\mathrm{~m} / \mathrm{s}$ & $\mathrm{y}$ component of velocity \\
\hline$w$ & $\mathrm{~m} / \mathrm{s}$ & $\mathrm{z}$ component of velocity \\
\hline$W$ & $\mathrm{~J}$ & Work \\
\hline$x$ & $\mathrm{~m}$ & Coordinate in wave-propagation direction \\
\hline$y$ & $\mathrm{~m}$ & $\begin{array}{l}\text { Coordinate perpendicular to the wave- } \\
\text { propagation direction }\end{array}$ \\
\hline$Z$ & $\mathrm{~Pa} \mathrm{~s} / \mathrm{m}^{3}$ & Acoustic impedance \\
\hline$z$ & $\mathrm{~m}$ & $\begin{array}{l}\text { Coordinate perpendicular to the wave- } \\
\text { propagation direction/ height }\end{array}$ \\
\hline
\end{tabular}




\section{Greek letters}

\begin{tabular}{|c|c|c|}
\hline Symbol & Unit & Signification \\
\hline$\alpha$ & $\operatorname{rad}$ & Phase angle \\
\hline$\beta$ & $\operatorname{rad}$ & Phase angle \\
\hline$\beta$ & - & Coefficient of nonlinearity \\
\hline$\Gamma$ & - & Dimensionless wave propagation constant \\
\hline$\gamma$ & - & Ratio of isobaric to isochoric specific heats \\
\hline$\Delta$ & - & Big difference \\
\hline$\delta$ & - & Small difference \\
\hline$\delta$ & $\mathrm{m}$ & Penetration depth \\
\hline$\epsilon$ & - & Porosity \\
\hline$\eta$ & - & Efficiency \\
\hline$\eta$ & - & $\begin{array}{l}\text { Dimensionless coordinate perpendicular to } \\
\text { wave propagation direction }\end{array}$ \\
\hline$\theta$ & $\mathrm{rad}$ & Angular coordinate \\
\hline$\lambda$ & $\mathrm{m}$ & Wave length \\
\hline$\mu$ & $\mathrm{kg} /(\mathrm{m} \mathrm{s})$ & Dynamic viscosity \\
\hline$v$ & $\mathrm{~m}^{\wedge} 2 / \mathrm{s}$ & Kinematic viscosity \\
\hline$\xi$ & - & $\begin{array}{l}\text { Dimensionless coordinate in wave- } \\
\text { propagation direction }\end{array}$ \\
\hline$\xi_{1}$ & $\mathrm{~m}$ & Displacement amplitude \\
\hline$\rho$ & $\mathrm{kg} / \mathrm{m}^{\wedge} 3$ & Density \\
\hline$\overline{\bar{\tau}}$ & $\mathrm{Pa}$ & Stress tensor \\
\hline$\Phi$ & $1 / \mathrm{s}^{\wedge} 2$ & Viscous dissipation function \\
\hline$\omega$ & $1 / \mathrm{s}$ & Angular frequency \\
\hline$\chi$ & - & Dimensionless radius \\
\hline
\end{tabular}




\section{Subscripts}

\begin{tabular}{cl}
\hline Symbol & Signification \\
\hline$c d$ & Cross direction \\
$p d$ & Propagation direction \\
$r e f$ & Reference \\
$C$ & Cold \\
$H$ & Hot \\
$s$ & Static \\
$h$ & Hydraulic \\
0 & Independent of time \\
1 & First order quantity (usually a complex \\
2 & amplitude) \\
$\kappa$ & Second order quantity \\
$v$ & Thermal \\
+ & Fiscous \\
- & Forward traveling wave \\
\hline
\end{tabular}

\section{Special symbols}

\begin{tabular}{cl}
\hline Symbol & Signification \\
\hline $\operatorname{Im}()$ & Imaginary part \\
$\operatorname{Re}()$ & Real part \\
$*$ & Complex conjugated \\
$=$ & Matrix \\
\langle\rangle & Cross sectionally averaged \\
$\rightarrow$ & Vector \\
\hline
\end{tabular}





\section{Introduction}

This simple engine is transforming heat into a strong acoustic sound without any moving part.

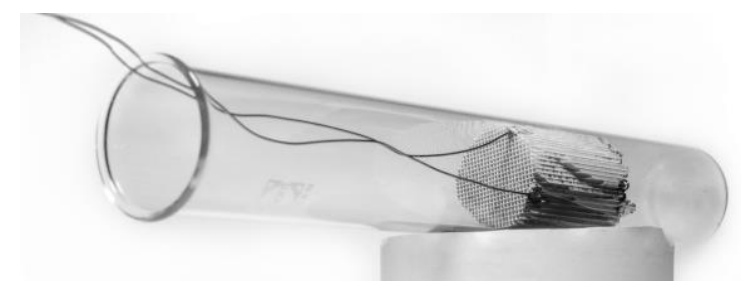

Figure 1.1: Thermoacoustic laser that converts heat from an electrically heated wire into a strong acoustic sound without any moving part.

Experiencing this sound four years ago puzzled me that much that I wanted to study this phenomenon in details. The underlying phenomenon is called thermoacoustics and according to Nikolaus Rott [1], who introduced this term in 1980, the term is "rather selfexplanatory", namely the combination of heat transfer and acoustics.

Acoustic waves are commonly described as a combination of pressure and velocity changes, but as waves propagate adiabatically in free space, the temperature has to change accordingly:

$$
\frac{T_{1}}{T_{0}}=\frac{\gamma-1}{\gamma} \frac{p_{1}}{p_{0}}
$$

While these temperature changes are hardly noticeable in open space, they can be used in thermoacoustic engines like the one given above to create a strong sound. The first qualitative explanation of this phenomenon was given by Lord Rayleigh in 1887 [2], who recognized that "if heat is given to the air at the moment of largest compression or taken from it at the moment of largest expansion, the acoustic wave is encouraged".

This is exactly what happens in the porous material of the thermoacoustic device shown in Figure 1.1. But next to the desired conversion of energy described above, thermoacoustics can also have a large harmful effect. In gas turbines, for example, the thermoacoustic effect can excite the resonance mode of the combustion chamber and destroy the latter within a short time [3]. In gas turbines the thermoacoustic resonance has to be avoided by an appropriate design and combustion control strategy [4]. These necessary design steps endorse the power that the thermoacoustic effect can have when applied in a proper way.

The work in this thesis does not focus on the part of thermoacoustics related to combustion and where thermoacoustic has a destructive nature. Instead, it focuses on applications like the thermoacoustic laser presented above, where the thermoacoustic effect is used for energy conversion. In these thermoacoustic devices the acoustic wave interacts 
with a close by solid, which is subject to a temperature gradient. Large advances in modeling this effect were achieved by Rott from 1969 on and his work led to a quantitative understanding of the phenomenon [5]. Later, Swift reviewed and summarized the thermoacoustic theory in his book [6]. The derived theory is still used for the design of thermoacoustic devices, for example in the one-dimensional code DeltaEC [7], which is largely used in the thermoacoustic community $[8,9,10]$.

\subsection{Thermoacoustic devices}

The aforementioned one-dimensional theory led to the development of numerous thermoacoustic devices. An important first step was the development of a standing wave thermoacoustic refrigerator by Hofler [11], who used a closed resonator and a loudspeaker in order to create a temperature gradient in the porous material. This means that instead of converting heat into acoustic power, like in the case of the thermoacoustic laser, the acoustic wave pumped the heat and created a temperature gradient. Numerous other devices were build using the same underlying theoretical thermodynamic cycle (for example [12]), but all suffer from the fact that an imperfect thermal contact is needed such that the phasing between the acoustic wave and the heat transfer fulfils the Rayleigh criterion. This imperfect thermal contacts leads to low theoretic efficiencies.

An important step in the development of thermoacoustic devices was the understanding of Cerperley that thermoacoustic devices based on traveling waves could reach much higher theoretical efficiencies, as in theory the underlying thermodynamic cycle is a Stirling cycle [13]. The first traveling wave thermoacoustic Stirling engines developed by Swift [14] and de Blok [15] at about the same time were a milestone in thermoacoustics. The Swift type of engine was designed with DeltaEC and reached an efficiency of 30\% from heat to acoustic power, without any moving part [9]. A schematic illustration of this type of engine is given in Figure 1.2.

Next to the promise of high theoretic efficiencies, thermoacoustic devices possess further advantages. One of these advantages is that the thermoacoustic effect occurs without any moving part, which is especially useful for cryogenic cooling, as high tolerances in the cold part can be avoided [16]. Another advantage is that most thermoacoustic devices use noble gases like helium or air, which are not harmful for the environment [6]. Furthermore, thermoacoustic devices can be supplied with any form of heat, which makes them suitable for regenerative energy sources, like solar energy [17]. Even small temperature differences can be used, such that waste heat can be upgraded [18]. This makes thermoacoustics a promising technology for energy conversion.

Using the specific advantages of thermoacoustics, numerous specific applications have been investigated in the recent years. One of these innovative applications, where the reliability of the device plays an important role, is the thermoacoustic self-powered temperature sensor and telemetry system for application in nuclear power plants investi- 
gated by Ali et al. [19]. The device uses a standing wave device which emits sound of a defined frequency which is characteristic for the temperature in the nuclear fuel rod.

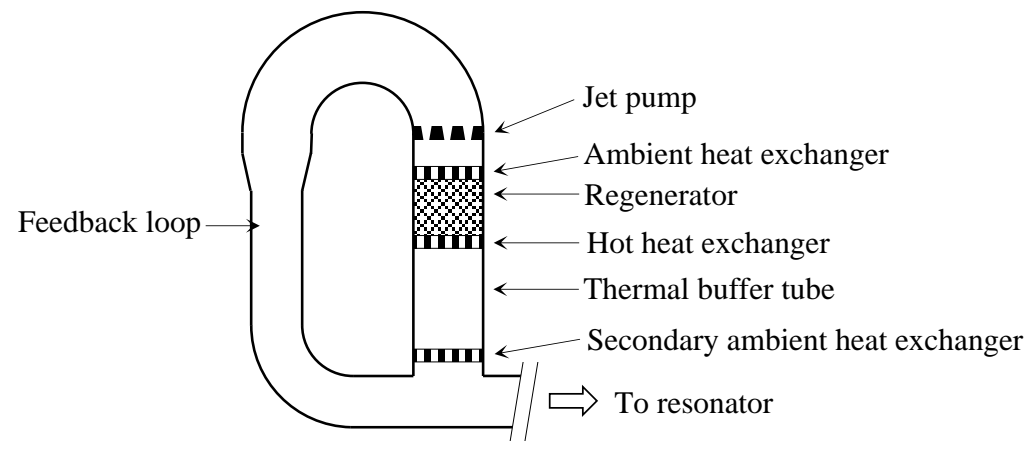

Figure 1.2: Schematic illustration of the traveling wave thermoacoustic engine [14].

Furthermore, thermoacoustics has been considered as small scale conversion technology for rural and developing areas. The reason is that while the physics behind the thermoacoustic converter is rather complex, the final implementation is simple, which allows for low production, investment and maintenance costs [20, 21]. Another field investigated in numerous studies for industrial application is waste heat recovery, as already small temperature differences can be exploited with the thermoacoustic effect [22, 23, 24].

Despite all the thermoacoustic research done, numerous questions stay open and have to be answered in order to come closer to the theoretically predicted efficiencies [8]. Profound knowledge lacks, especially when the flow field loses its one-dimensional character and the one-dimensional equations are not valid anymore. This is the case for example for complex geometries, when the size of the thermoacoustic device shall be reduced or when different components interact with each other. Revealing the flow field inside the different components can lead to a better understanding of the underlying flow phenomena and thus to an improvement of the thermoacoustic devices.

\subsection{CFD modeling in thermoacoustics}

In order to get a better understanding of the flow field and the acoustic losses inside of a thermoacoustic component, flow visualization can be a useful tool. Different approaches can be used to visualize the flow inside of a thermoacoustic device. One possible approach is to use experimental flow visualization techniques like Particle Image Velocimetry (PIV). These experimental techniques have been applied successfully to visualize the vortex generation at the extremities of a stack (for example [25, 26, 27]), but are restricted to certain geometries by the laser and are time consuming and expensive. Another approach, which is used in this thesis, is to numerically model the flow field with Computational Fluid Dynamics (CFD). CFD simulations and PIV measurements are 
found to be in close agreement [27], but CFD simulations have the advantage that they allows revealing the entire flow field with all its physical quantities.

A large number of CFD publications in thermoacoustics focuses on the so called thermoacoustic couple, which is a parallel plate stack with heat exchangers at both extremities, modeled in different degrees of complexity. Cao et al. [28] were the first to introduce the thermoacoustic couple. They modeled it as one plate with zero wall thickness and no heat exchangers. The acoustic wave was introduced by imposing the velocity at both sides of the plate. Later, Worlikar et al. extended the stack model to a finite plate thickness and implemented heat exchangers at both extremities of the stack [29, 30, 31, 32]. In a further step, Besnoin et al. investigated the placement of the heat exchangers relative to the stack plate $[33,34]$. Marx et al. applied a boundary condition which uses the method of characteristics in order to introduce an acoustic wave that propagates inside of the domain, while the waves from inside of the domain can leave without reflections. With this boundary condition they investigated the thermoacoustic couple and revealed the nonlinearities in the temperature and velocity [35, 36, 37, 38]. All the previous mentioned works were conducted with dedicated codes developed for the respective specific application. Zoontjens et al. made the step towards commercially available CFD software, namely ANSYS Fluent [39], in order to model the thermoacoustic couple [40].

Besides the simulations of the thermoacoustic couple, simulations of entire engines have also been performed. Lycklama à Nijeholt et al. performed simulation of a coaxial traveling wave thermoacoustic engine with ANSYS CFX [41], from which they concluded that CFD can be used for the prediction and optimization of thermoacoustic devices, as the agreement between the simulations and the experiments was satisfactory [42]. In their work they did not geometrically resolve the heat exchanger and the regenerator, but modeled it with help of volume sources in order to reduce the computational resources need. Scalo et al. extended this model to the third dimension and added a secondary heat exchanger in order to reach a limit cycle [43].

Another work of an entire engine but with a different geometry is investigated by Zink at al. [44]. They modeled an entire standing wave engine in two dimensions and investigated the influence of a coiled resonator. They have shown that a bent resonator, which cannot be easily investigated with one-dimensional models, reduces the efficiency of the device and shifts the resonance frequency.

This increasing number of thermoacoustic CFD simulations shows that CFD is seen as a valid and promising method to gain a better understanding of the flow phenomena that are beyond the one-dimensional thermoacoustic equations. From the earlier named works one can also see that an increasing number of numerical studies is conducted with commercial CFD codes, which is promising as these codes are readily available to a large public and allow for broad investigations. 
In general, two different approaches for the thermoacoustic modeling in CFD can be differentiated from the earlier named work. The first approach consists in modeling entire engines. This approach allows investigating the time dependent behavior of the thermoacoustic device and the interaction between the different parts, without using a dedicated acoustic boundary condition. On the other side, this is computationally expensive and geometric simplifications are needed to model all the scales comprised in the device. This makes it difficult to conduct a study over a large amount of parameters that is often necessary to get a profound knowledge of the underlying flow phenomena.

The second approach reduces the study to only one component of the thermoacoustic device, like for example the thermoacoustic couple presented above. The disadvantage of this method is that dedicated boundary conditions are needed for CFD, as the pressure and velocity are imposed at the boundary and these quantities are a superposition of an acoustic wave from outside of the domain and one from the inside of the domain. These dedicated boundary conditions are not yet readily available in the commercial CFD packages, but once they are introduced, detailed investigation of an isolated component can be done. As the computational domain is reduced compared to the entire device simulations, a large parameters space can be investigated to get in-depth knowledge of the flow field in the respective components. This is the reason that in this thesis acoustic boundary conditions are implemented into the commercially available CFD code ANSYS Fluent [39] and an in-depth validation is performed. In a second step it is shown that it is possible to study the geometric parameters of components detached from the rest of the thermoacoustic device. This will open the field to a large number of in-depth numerical thermoacoustic studies in the future.

\subsection{Thesis outline}

The aim of this research is to make a next step towards numerical simulations of thermoacoustic components with commercially available CFD software. First, in Chapter 2 the general principle of thermoacoustics and the corresponding theory are summarized. The numerical method used in this thesis is described in Chapter 3, including the implemented non-reflecting boundary conditions needed in order to reduce the computational domain to a thermoacoustic component. In the subsequent Chapter 4 the numerical method and especially its boundary conditions are validated against the low reduced frequency solutions derived in Chapter 2. The necessary discretization and the best numerical parameters for the ensuing simulations are derived. With these optimal numerical parameters known, four different components of a thermoacoustic device, where the non-linear effects cannot be neglected, are studied in Chapter 5.

The first investigated component is the thermal buffer tube. Its aim is to provide thermal insulation between the hot heat exchanger and the secondary ambient heat exchanger while transmitting the acoustic power out of the hot zone. But due to the interaction of the acoustic wave with the temperature gradient, a two-dimensional steady mass 
flux called acoustic streaming occurs, which leads to unwanted thermal losses. In Chapter 5.1 the streaming patterns inside the thermal buffer tube are revealed and the resulting temperature profiles are given.

The second investigated component is the U-bend that feeds back the acoustic wave in a traveling wave device. In the scope of reducing the size of the thermoacoustic devices, the bend gets increasingly sharp, introducing additional losses. In Chapter 5.2 the influence of the geometric parameters of the bend on the flow field are investigated. The deviations from the low reduced frequency model are revealed and for even sharper bends the limit to vortex generation is given.

In the subsequent Chapter 5.3 an experimental method to calculate the thermoacoustic functions is applied to CFD. The thermoacoustic functions represent the spatially averaged diffusion functions for heat and momentum and allow incorporating threedimensional effects into the one-dimensional thermoacoustic equations. The method is applied to a reduced model of a stacked screen regenerator and the thermoacoustic functions are given for different geometries of the regenerator model.

In Chapter 5.4 the non-linear entrance effects at a stacked screen regenerator are investigated and the resulting changes in the mean temperature profile are given for different geometric variations.

The thesis ends with concluding remarks in Chapter 6. 


\section{Thermoacoustics}

This chapter gives a short summary of the thermodynamics and fluid mechanics upon which thermoacoustic theory is based. In Chapter 2.1 the thermodynamic aspects of thermoacoustic devices are discussed to provide a general understanding. A qualitative description is then given in the following subchapters. In Chapter 2.2 the conservation equations of fluid mechanics are used to derive the general thermoacoustic equations. In Chapter 2.3 the flow field is derived for some specific cases.

\subsection{Thermodynamics}

It is possible by applying the first law of thermodynamics to thermoacoustic devices to estimate their performance. The subsequent subchapters will expose the thermodynamic cycle that thermoacoustic devices undergo.

\subsubsection{Laws of thermodynamics}

The energy flow and the performance of thermoacoustic devices can be described by looking at the first law of thermodynamics for an open system [45]:

$$
\begin{aligned}
\frac{d E_{\text {sys }}}{d t}=\sum_{i} \dot{Q}_{i} & +\sum_{j} \dot{W}_{j}+\sum_{\text {in }} \dot{m}_{\text {in }}\left(h_{\text {in }}+g z_{\text {in }}+\frac{1}{2} \vec{v}_{\text {in }}^{2}\right) \\
& -\sum_{\text {out }} \dot{m}_{\text {out }}\left(h_{\text {out }}+g z_{\text {out }}+\frac{1}{2} \vec{v}_{\text {out }}^{2}\right)
\end{aligned}
$$

Depending on the energy flow, two different types of devices can be distinguished: thermoacoustic prime movers and heat pump. In both cases, it can be assumed that the system is closed and that it has reached a time averaged steady state. For a prime mover equation (2.1) can be simplified to:

$$
0=\dot{Q}_{H}-\dot{Q}_{c}-\dot{W}
$$

Heat is taken from a heat source at a higher temperature $T_{H}$ and exhausted at a lower temperature $T_{C}$ to a heat sink while producing acoustic work (see Figure $2.1 \mathrm{a}$ )). The performance of the prime mover can be estimated by dividing the useful acoustic power $\dot{W}$ by the heating power $\dot{Q}_{H}$, which gives the efficiency of the prime mover:

$$
\eta=\frac{\dot{W}}{\dot{Q}_{H}}
$$

For refrigerators and heat pumps equation (2.1) can be simplified to:

$$
0=-\dot{Q}_{H}+\dot{Q}_{c}+\dot{W}
$$

Heat is thus absorbed at a lower temperature $T_{C}$ and rejected at a higher temperature $T_{H}$ while consuming acoustic work (see Figure $2.1 \mathrm{~b}$ )). As refrigerators and heat pump have different goals, their performances have to be quantified differently. For a refrigera- 
tor the goal is to extract heat at a lower temperature with as little work as possible. The coefficient of performance can be calculated as:

$$
\operatorname{COP}_{\text {ref }}=\frac{\dot{Q}_{C}}{\dot{W}}
$$

The useful output of a heat pump is the heat rejected at a higher temperature $T_{H}$ while it consumes acoustic power. The coefficient of performance for a heat pump can be defined as:

$$
\operatorname{COP}_{h p}=\frac{\dot{Q}_{H}}{\dot{W}}
$$

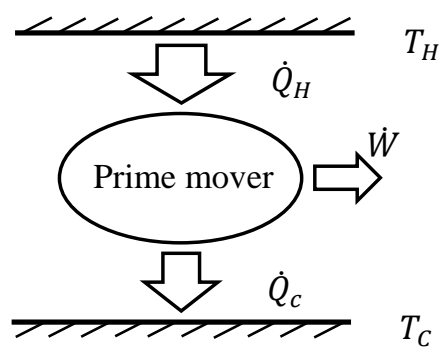

a) prime mover

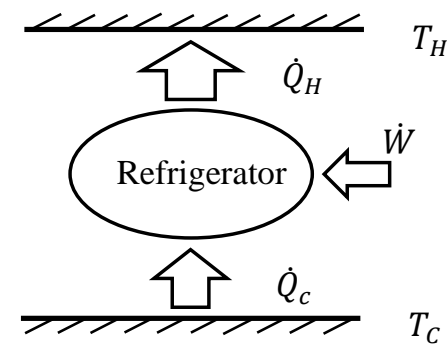

b) refrigerator / heat pump

Figure 2.1: Thermodynamic representation of the heat and work flow inside a) a prime mover and b) a refrigerator or heat pump [46].

\subsubsection{Thermodynamic cycles}

Thermoacoustic devices can also be classified by their underlying thermodynamic process. Two different types are distinguished depending upon the phasing between the oscillation in pressure and velocity. When the pressure and the velocity oscillations are out of phase, the phasing is referred to as standing wave, while when the pressure and velocity are in phase the phasing is referred to as traveling wave. The thermodynamic process for both cases is presented in the following subchapters.

\subsubsection{Standing wave phasing}

For standing wave phasing the pressure and the velocity oscillations are out of phase. A gas parcel will be compressed and at the same moment displaced in positive direction (see Figure 2.2 A-B). Half a period later, during the expansion, it will be displaced in the negative direction (see Figure 2.2 C-D). When the distance of a gas parcel to the wall is much larger than the thermal penetration depth $\left(y \gg \delta_{k}\right.$ see Figure 2.3 a) ) an adiabatic cycle will occur and no heat will be exchanged between the bulk of the fluid and the wall. 
This adiabatic cycle is summarized in the $p$-v-diagram in Figure $2.3 \mathrm{~b}$ ). The gas parcel temperature also changes during the adiabatic cycle, due to the changing pressure. When the gas parcel temperature is plotted over the particles position, the so called critical temperature gradient can be obtained, which is an important quantity for standing wave engines as will be shown.

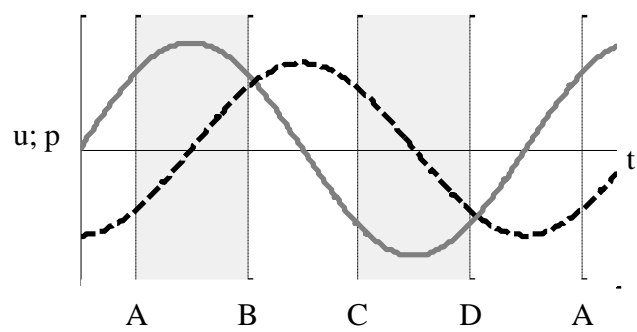

Figure 2.2: $\quad$ Pressure (black dashed line) and velocity (solid gray line) over time for a standing wave. Four stages can be distinguished: A-B, the fluid is displaced to the right and compressed; B-C, The fluid stays at its right extremity; C-D, the fluid is displaced to the left and expanded; D-A, the fluid stays at its left extremity. [46]

If the gas parcel is in close contact to a wall subject to a temperature gradient $(\mathrm{y} \ll$ $\delta_{\mathrm{k}}$ see Figure $\left.2.3 \mathrm{a}\right)$ ) the gas parcel temperature follows the wall temperature. Two cases can be distinguished, depending on the temperature gradient of the wall. If the temperature gradient is higher than the critical temperature gradient, the gas parcel will be heated during the compression (see Figure 2.2 A-B), while if the temperature gradient is smaller than the critical temperature gradient the gas parcel will be cooled. As compression and heat exchange happen simultaneously, no surface is enclosed in the $p$ - $v$-diagram in Figure $2.3 \mathrm{c}$ ) and hence no work is done or consumed.

If the distance of the gas parcel to the wall is on the same order of magnitude as the thermal penetration depth, a time delay between the compression and the heat transfer occurs. Depending on whether the temperature gradient of the wall is higher or lower than the critical temperature gradient, the device is working as a prime mover or refrigerator, respectively. In case of a prime mover, the cycle that the gas parcel undergoes can be approximated as follows:

1) compression $(\mathrm{A}-\mathrm{B})$

2) isobaric heating (B-C)

3) expansion (C-D)

4) isobaric cooling (D-A)

These four steps correspond to the idealized Brayton cycle [47], which is shown for the prime mover and refrigerator in Figure $2.3 \mathrm{~d}$ ) and e), respectively. As a surface is enclosed in the p-v diagram, work is exchanged with the environment. 
The thermoacoustic component in which the thermoacoustic effect occurs in standing wave devices is called a stack. It is a porous structure, frequently made of parallel plates [6], which has pores on the order of a few thermal penetration depths.

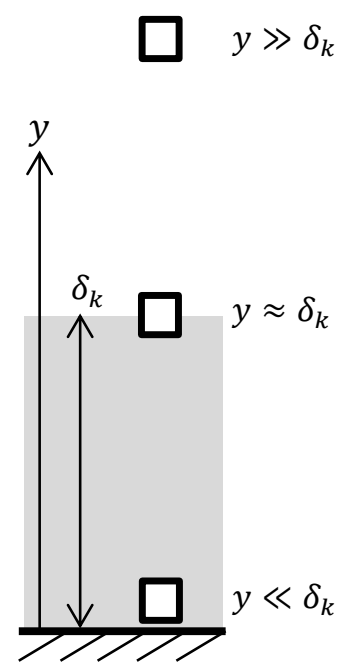

a)

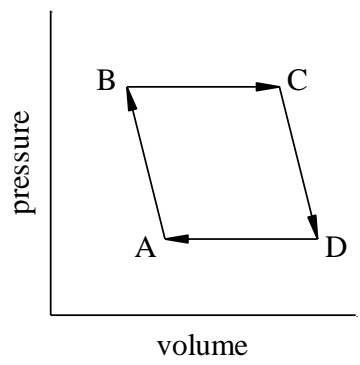

d) $y \approx \delta_{k}$ : prime mover

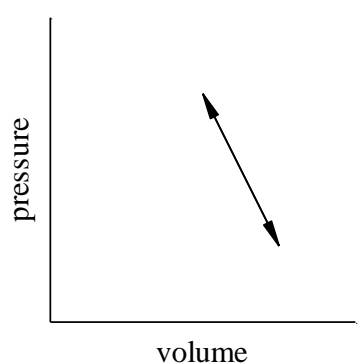

b) $y \gg \delta_{k}$

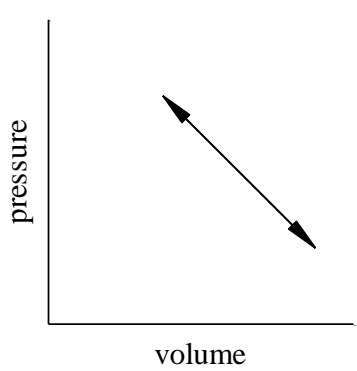

c) $y \ll \delta_{k}$

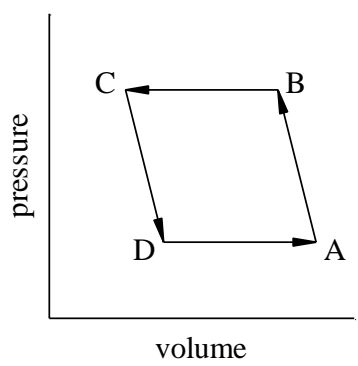

e) $y \approx \delta_{k}$ : refrigerator

Figure 2.3: $\quad$ Definition of the position y between a wall with temperature gradient and gas parcels subject to an acoustic standing wave a) and the corresponding p-v diagrams of the gas parcels b)-e) [46]. 


\subsubsection{Traveling wave phasing}

In traveling acoustic waves the pressure and velocity oscillation are in phase. A gas parcel undergoes the following cycle: compression (see Figure 2.4 A-B); displacement to the right (B-C); expansion (C-D); displacement to the left (D-A). When the gas parcel is in ideal contact with a wall subject to a positive temperature gradient, the gas parcel undergoes the following cycle:

1) isothermal compression (A-B)

2) isochoric heating (B-C)

3) isothermal expansion (C-D)

4) isochoric cooling (D-A)

This cycle is known as the idealized Stirling cycle [47] and is summarized in Figure 2.5 a). It is a clockwise cycle, thus acoustic power is produced. When the temperature gradient is negative, the heating and cooling phase are interchanged. Thus, the gas parcel undergoes a counterclockwise cycle (Figure $2.5 \mathrm{~b}$ )) and acoustic power is consumed. In this case the acoustic power is used to pump heat. Depending on the aim, the thermoacoustic device is working as a refrigerator or heat pump.

The thermoacoustic component, in which the thermoacoustic effect occurs in a traveling wave device, is called a regenerator. It is a porous part, which is frequently assembled from stacked woven or random fiber screens [6], as these provide a wide selection of useful pore sizes. Compared to the stack in standing wave devices the pores size is smaller in the regenerator. The pore size of the regenerator is an important characteristic that should be selected to assure perfect thermal contact between the gas and the solid, while balancing the associated viscous losses.

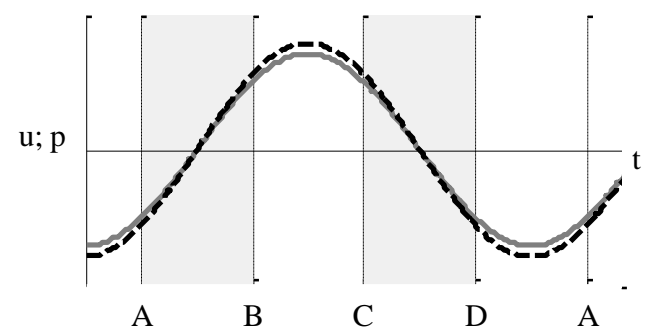

Figure 2.4: Pressure (black dashed line) and velocity (solid gray line) over time for standing wave phasing. Four stages of the gas parcel can be distinguished: $\mathrm{A}-\mathrm{B}$, compression; $\mathrm{B}-\mathrm{C}$, displacement to the right; $\mathrm{C}-\mathrm{D}$, expansion; D-A, displacement to the left. [46] 


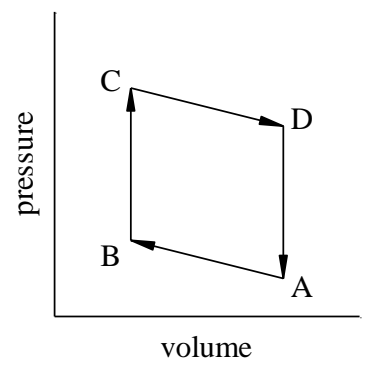

a) prime mover

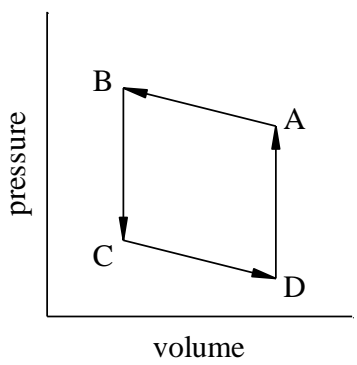

b) refrigerator

Figure 2.5: Thermodynamic cycle of gas parcel that is subject to a traveling wave phasing, close to a wall. a) Prime mover: wall has a positive temperature gradient, acoustic power is produced; b) refrigerator: wall has a negative temperature gradient, acoustic power is consumed. [46]

\subsubsection{Bucket-brigade effect}

In a regenerator or stack a large amount of gas parcels undergo the aforementioned thermodynamic cycles. As the displacement amplitude of the gas parcels is usually only a fraction of the total regenerator or stack length [6], the gas parcels absorb heat at one place and release it at another place, passing the heat from one parcel to another. Figure 2.6 shows the case of a prime mover where every gas parcel takes part in transferring the heat from the hot end to the cold end, while producing acoustic power. From the analogy with fire workers standing in a line passing the buckets of water from one to the other, this effect is called the Bucket-brigade effect.

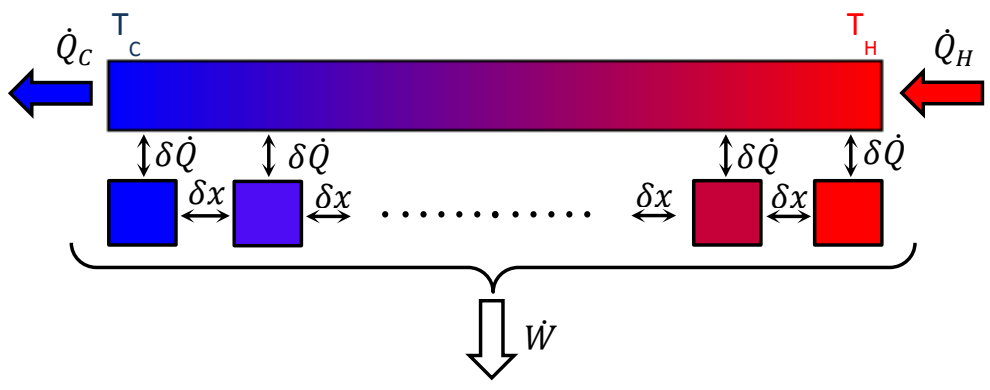

Figure 2.6: $\quad$ Bucket-brigade effect in the case of a prime mover. Heat is passed from one gas parcel to the other while producing acoustic power [12].

\subsection{General thermoacoustic theory}

The thermoacoustic effect was described in the previous chapter in a qualitative way in order to provide a general idea. In this chapter quantitative equations to describe the 
thermoacoustic effect will be derived from the conservation equations. Introducing assumptions valid for the specific case of thermoacoustics allows one to simplify the full Navier-Stokes equations to one-dimensional differential equations. These equations can be solved effectively with programs like DeltaEC [7] and Sage [48] and are a useful tool for the design of thermoacoustic engines and heat pumps $[8,9,10]$. The underlying linear theory was derived by Rott in the seventies [1] and reviewed later by Swift [6]. A short derivation of the thermoacoustic equations will be given in this chapter in order to introduce the thermoacoustic functions. In Chapter 2.2.1 the full conservation equations are given, together with the equations of state. The complete set of equations is then linearized and Fourier transformed in Chapter 2.2.2. Finally in Chapter 2.2.3 the thermoacoustic equations are obtained and the thermoacoustic functions are introduced. Further discussion about the thermoacoustic function is given in Chapter 2.2.4.

\subsubsection{Conservation equations}

The basic equations governing the thermoacoustic effect are derived using the fact that mass, momentum and energy are conserved. The conservation equations are derived by making a balance of the conserved quantities over an infinitesimal control volume. The changes of the conserved quantities can be obtained from the fluxes through the borders of the control volume. These equations are known as the Navier-Stokes equations [49]:

- Mass conservation:

$$
\frac{\delta \rho}{\delta t}+\nabla \cdot(\rho \vec{v})=0
$$

- Momentum conservation:

$$
\frac{\delta}{\delta t}(\rho \vec{v})+\nabla \cdot(\rho \vec{v} \vec{v})=-\nabla p+\nabla \cdot(\overline{\bar{\tau}})+\rho \vec{g}
$$

where $\vec{g}$ is the gravity and $\overline{\bar{\tau}}$ is the viscous stress tensor. Assuming a Newtonian fluid the stress tensor is given as:

$$
\overline{\bar{\tau}}=\mu\left(\left(\nabla \vec{v}+\nabla \vec{v}^{T}\right)-\frac{2}{3} \nabla \cdot \vec{v} I\right)
$$

- Energy conservation, assuming Fourier law for heat conduction and a Newtonian fluid:

$$
\rho \frac{\partial e}{\partial t}+\rho \vec{v} \vec{\nabla} e=\vec{\nabla} \cdot(k \vec{\nabla} T)-p \cdot \vec{\nabla} \vec{v}+\mu \Phi
$$

where $e$ is the specific internal energy and $\Phi$ the viscous dissipation function, which can be written in a Cartesian coordinate system as:

$$
\begin{aligned}
\Phi=2 \cdot\left[\left(\frac{\partial u}{\partial x}\right)^{2}+\right. & \left.\left(\frac{\partial v}{\partial y}\right)^{2}+\left(\frac{\partial w}{\partial z}\right)^{2}\right]+\left(\frac{\partial v}{\partial x}+\frac{\partial u}{\partial y}\right)^{2}+\left(\frac{\partial w}{\partial y}+\frac{\partial v}{\partial z}\right)^{2} \\
& +\left(\frac{\partial u}{\partial z}+\frac{\partial w}{\partial x}\right)^{2}-\frac{2}{3}\left(\frac{\partial u}{\partial x}+\frac{\partial v}{\partial y}+\frac{\partial w}{\partial z}\right)^{2}
\end{aligned}
$$


In order to close the system of equations, two additional equations are needed. These are the state equations. Throughout this thesis it will be assumed that the working fluid behaves like an ideal gas and follows the ideal gas law:

$$
p=\rho R_{s} T
$$

The second equation needed to close the system is the caloric equation of state for an ideal gas:

$$
d e=c_{v} d T
$$

Assuming the specific heat capacities to be constant, the energy equation can finally be written in terms of temperature as:

$$
c_{v} \rho \frac{\partial T}{\partial t}+c_{v} \rho \vec{v} \vec{\nabla} T=\vec{\nabla} \cdot(k \vec{\nabla} T)-p \cdot \vec{\nabla} \vec{v}+\mu \Phi
$$

All together equations (2.7), (2.8), (2.12) and (2.14) form a complete set of equation that can be solved for the pressure, the velocity, the temperature and the density.

\subsubsection{Linearization and Fourier transformation}

The conservation equations and the equations of state given in Chapter 2.2.1 are valid for a large variety of flows. Solving these equations is difficult and no general solution exists. Assumptions are necessary to solve the complete flow field. For this reason the flow variables are rewritten assuming the small signal approximation, valid for a large amount of acoustic cases [50]:

$$
\begin{gathered}
p=p_{0}+p^{\prime}(x, t) \\
v=\vec{v}^{\prime}(x, t) \\
T=T_{0}+T^{\prime}(x, t) \\
\rho=\rho_{0}+\rho^{\prime}(x, t)
\end{gathered}
$$

The index zero indicates a mean value, which is only dependent on the wave propagation direction, the $x$-coordinate in Cartesian coordinates. The prime indicates the small variations, in time and space. The underlying assumption for the small signal approximation can be rewritten as [50]:

$$
\left|\vec{v}^{\prime}(x, t)\right| \ll c_{0}
$$

where $c_{0}$ is the unperturbed speed of sound.

Higher order terms are products of small quantities, they are thus small and can be neglected. It follows that the convective effects and the viscous dissipation in the energy equation can be neglected. This means that viscous effects are still taken into account in the momentum equation, but do not lead to a temperature increase of the fluid. The resulting linearized equations are:

$$
\begin{gathered}
\frac{\delta \rho^{\prime}}{\delta t}+\nabla \cdot\left(\rho_{0} \vec{v}^{\prime}\right)=0 \\
\rho_{0} \frac{\delta}{\delta t}\left(\vec{v}^{\prime}\right)=-\nabla p^{\prime}+\nabla \cdot\left(\overline{\bar{\tau}}^{\prime}\right)+\rho_{0} \vec{g}
\end{gathered}
$$




$$
\begin{gathered}
c_{p} \rho_{0}\left(\frac{\partial T^{\prime}}{\partial t}+u_{1} \frac{d T_{0}}{d x}\right)=k \Delta \mathrm{T}+\frac{\partial p^{\prime}}{\partial t} \\
\frac{p^{\prime}}{p_{0}}=\frac{\rho^{\prime}}{\rho_{0}}+\frac{T^{\prime}}{T_{0}}
\end{gathered}
$$

Assuming that the system is steady periodic, equation (2.20) to (2.23) can be transformed to the frequency domain by applying the Fourier transformation for the angular frequency $\omega[51]$ :

$$
F(\omega)=\frac{1}{\sqrt{2 \pi}} \int_{-\infty}^{\infty} f^{\prime}(t) e^{-i \omega t} d t
$$

it follows:

$$
\begin{gathered}
i \omega \rho_{1}+\nabla \cdot\left(\rho_{0} \vec{v}_{1}\right)=0 \\
i \omega \rho_{0} \vec{v}_{1}=-\nabla p_{1}+\nabla \cdot\left(\overline{\bar{\tau}}_{1}\right)+\rho_{0} \vec{g} \\
c_{p} \rho_{0}\left(i \omega T_{1}+u_{1} \frac{d T_{0}}{d x}\right)=k \Delta \mathrm{T}+i \omega p_{1} \\
\frac{p_{1}}{p_{0}}=\frac{\rho_{1}}{\rho_{0}}+\frac{T_{1}}{T_{0}}
\end{gathered}
$$

This set of equations can be solved for discrete frequencies independently of each other as it consists of linear equations. The solution for each frequency can then be added. The flow variables in the Fourier domain can be transformed back to the time domain by the following transformation:

$$
\begin{gathered}
p=p_{0}+\operatorname{Re}\left(p_{1} \mathrm{e}^{i \omega t}\right) \\
v=\operatorname{Re}\left(v_{1} \mathrm{e}^{i \omega t}\right) \\
T=T_{0}+\operatorname{Re}\left(T_{1} \mathrm{e}^{i \omega t}\right) \\
\rho=\rho_{0}+\operatorname{Re}\left(\rho_{1} \mathrm{e}^{i \omega t}\right)
\end{gathered}
$$

\subsubsection{Thermoacoustic equations}

With additional assumptions, the thermoacoustic equations can be obtained. The effect of gravity is neglected. In most thermoacoustic devices the characteristic length $l_{c d}$ in the cross-direction is much smaller than the wavelength. As the ratio of these two quantities is defined as the reduced frequency $k$, this assumption can be written as:

$$
k=\frac{\omega l_{c d}}{c_{0}} \ll 1
$$

From this assumption it follows that the gradients in the cross-direction are much higher than in the wave propagation direction. Applying the low reduced frequency approximation to the momentum equation in the cross-direction, it follows that the pressure amplitude is only dependent on the $\mathrm{x}$-coordinate. With this assumption the linearized momentum equation (2.26) can be rewritten for wave propagation in the $x$-direction: 


$$
i \omega \rho_{0} u_{1}=-\frac{\partial p_{1}}{\partial x}+\mu\left(\frac{\partial^{2} u_{1}}{\partial y^{2}}+\frac{\partial^{2} u_{1}}{\partial z^{2}}\right)
$$

Integrating this equation over the cross-direction leads to the first thermoacoustic equation in terms of the volume flow rate $U_{1}$ :

$$
U_{1}=\frac{i}{\omega \rho_{0} A}\left(1-f_{v}\right) \frac{d p_{1}}{d x}
$$

where $f_{v}$ is the viscous thermoacoustic function, which is a geometric function depending on the frequency and will be discussed in more details in the following Chapter.

The second thermoacoustic equation can be derived from the two remaining conservation equations. Integrating the continuity equation (2.20) over the cross-section and applying the divergence theorem assuming no-slip at the wall yields:

$$
i \omega A\left\langle\rho_{1}\right\rangle+\frac{d}{d x}\left(\rho_{0} U_{1}\right)=0
$$

The low reduced frequency approximation is also applied to the energy equation (2.27), which leads to:

$$
c_{p} \rho_{0}\left(i \omega T_{1}+u_{1} \frac{d T_{0}}{d x}\right)=k\left(\frac{\partial^{2} T_{1}}{\partial y^{2}}+\frac{\partial^{2} T_{1}}{\partial z^{2}}\right)+i \omega p_{1}
$$

Assuming that the wall is isothermal $\left(T_{1}=0\right)$ and integrating over the cross-section leads to:

$$
\left\langle T_{1}\right\rangle=\frac{1-f_{\kappa}}{c_{p} c_{0}} p_{1}-\frac{1}{i \omega A} \frac{d T_{0}}{d x} \frac{\left(1-f_{\kappa}\right)-\operatorname{Pr}\left(1-f_{v}\right)}{\left(1-f_{v}\right)(1-\operatorname{Pr})} U_{1}
$$

where $f_{\kappa}$ is the thermal thermoacoustic function, which is a geometric function depending on the frequency and will be discussed in more details in the following Chapter. Inserting (2.38) and (2.28) into (2.36) leads to the second thermoacoustic equation:

$$
\frac{d U_{1}}{d x}=-\frac{i \omega A}{\gamma p_{0}}\left(1+(\gamma-1) f_{\kappa}\right) p_{1}+\frac{f_{\kappa}-f_{v}}{\left(1-f_{v}\right)(1-\operatorname{Pr})} \frac{1}{T_{0}} \frac{d T_{0}}{d x} U_{1}
$$

Using the first two thermoacoustic equations, the volume flow rate $U_{1}$ and the pressure $p_{1}$ can be obtained. The last unknown is the mean temperature gradient $d T_{m} / d x$. This gradient can be obtained by looking at the total energy flow $\dot{E}$, which is the sum of the enthalpy flow and heat flow:

$$
\dot{E}=\frac{1}{2} \rho_{0} \iint_{A} \operatorname{Re}\left(h_{1} u_{1}^{*}\right) d A-\left(A k+A_{\text {sol }} k_{\text {sol }}\right) \frac{d T_{0}}{d x}
$$

where $h_{1}$ is the specific enthalpy, $A_{s o l}$ the solid surface and $k_{s o l}$ the thermal conduction coefficient of the solid. Using the caloric equation of state and integrating over the cross-section yields the mean temperature gradient:

$$
\frac{d T_{0}}{d x}=\frac{\dot{E}-\frac{1}{2} \operatorname{Re}\left(p_{1} U_{1}^{*}\left(1-\frac{\left(f_{\kappa}-f_{v}^{*}\right)}{(1+P r)\left(1-f_{v}^{*}\right)}\right)\right)}{\frac{\rho_{0} c_{p}\left|U_{1}\right|^{2}}{2 \omega A(1-P r)\left|1-f_{v}\right|^{2}} \operatorname{Im}\left(f_{v}^{*}+\frac{f_{\kappa}-f_{v}^{*}}{1+P r}\right)-A k-A_{\text {sol }} k_{\text {sol }}}
$$


Altogether, equations (2.35), (2.39) and (2.41) form the set of thermoacoustic equations, which were derived using the following main assumptions:

- No mean velocity.

- $\quad$ The pressure, the velocity, the temperature and the density can be written as the sum of a mean value and a first order variation, where the variation part is much smaller than the mean value.

- The acoustic wave has only one fixed frequency and one propagation direction.

- Geometric changes in propagation direction are small.

- The system is steady periodic. This means that after one acoustic cycle the system is in the same state as at the beginning of the cycle.

\subsubsection{Thermoacoustic functions}

The thermoacoustic functions $f_{\kappa}$ and $f_{v}$ account for three-dimensional effects in the one-dimensional thermoacoustic equations. The time dependent three-dimensional flow field can be reduced to a one-dimensional frequency dependent problem. Physically speaking, the thermoacoustic functions are the spatially averaged diffusion functions for heat and momentum respectively:

$$
f_{j}=\iint_{A} h_{j} d A, \text { for } j=\kappa \text { or } v
$$

They are both dependent on the geometry, the material parameters and the frequency. The momentum diffusion function $h_{v}$ is defined by the following differential equation:

$$
h_{v}-\frac{\delta_{v}^{2} i}{2} \nabla_{c d}^{2} h_{v}=0
$$

which can be obtained from equation (2.34), assuming that the cross-directional effects are independent of the effects in the propagation direction. In this case the velocity can be written as (see also Chapter 2.3):

$$
u_{1}=\frac{i}{\omega \rho_{0}}\left(1-h_{v}(y, z)\right) \frac{d p_{1}}{d x}
$$

The boundary conditions for the momentum diffusion function $h_{v}$ are given through the boundary conditions of the velocity. The thermal thermoacoustic function $f_{\kappa}$ can be calculated from the heat diffusion function $h_{\kappa}$ according to equation (2.42). The heat diffusion function $h_{\kappa}$ has to solve a partial differential equation similar to the one given in Equation (2.43) with the viscous penetration depth $\delta_{v}$ replaced by the thermal penetration $\delta_{\kappa}$. The necessary boundary conditions can be obtained from the boundary conditions for the temperature oscillation at the wall [52]. The thermoacoustic functions can be obtained in three different ways. For some cases an analytical solution for the partial differential equations can be found. Some examples are given in Chapter 2.2.4.1. Another way to obtain the solution for complicated uniform pores is to numerically solve the differential equations (2.43). In Chapter 2.2.4.2 a numerical integration method implemented in MATLAB is presented. Finally Chapter 2.2.4.3 presents a method to experi- 
mentally estimate the thermoacoustic functions of stack or regenerator assemblies. This allows estimating the thermoacoustic functions of tortuous geometries using CFD. The obtained thermoacoustic functions can then be applied in the one-dimensional formalism.

\subsubsection{Existing analytical solutions}

For some geometries the thermoacoustic functions can be calculated analytically. The differential equation (2.43) can be solved assuming no-slip or isothermal boundaries at the walls for the viscous and the thermal part, respectively. The real and the imaginary part of the thermoacoustic functions are shown in Figure 2.7 for five geometries for which an analytical solution is known. These solutions are given in Appendix B. Figure 2.7 shows that for large ratios of $r_{h} / \delta$ the solution converges towards the boundary layer solution.

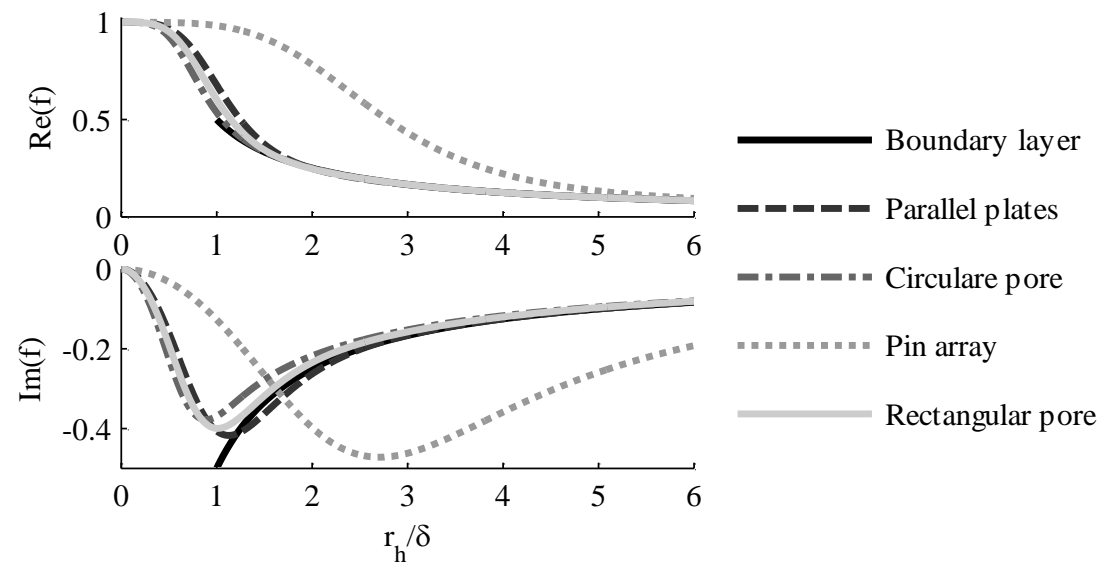

Figure 2.7: Thermoacoustic function $f$ for five geometries for which the analytical solution is known. Using $r_{h} / \delta_{v}$ on the horizontal axe yields $f_{v}$ and using $r_{h} / \delta_{\kappa}$ yields $f_{\kappa}$. With $r_{h}$ the hydraulic radius. The rectangle has an aspect ratio of 6:1. The pin array has $r_{o} / r_{i}=6[6]$.

\subsubsection{Numerical integration}

Another way to obtain the thermoacoustic functions is to numerically integrate the elliptic partial differential Equation (2.43) with its boundary conditions [53]. Solving for the different ratios of hydraulic radius to penetration depth, the thermoacoustic functions can be calculated as a function the dimensionless frequency even for complicated crosssections. An algorithm to solve Equation (2.43) was implemented in MATLAB using the boundary value solver. The algorithm was validated for different geometries for which an analytical solution exists, but it is possible to further extend the method to more complex geometries. The drawback of this method is that it cannot account for non-uniform pores. 


\subsubsection{Experimental estimation of the thermoacoustic functions}

In order to incorporate the complex flow field of real stacks and regenerators into the one-dimensional formulation, the thermoacoustic functions can be estimated experimentally. Two different experimental methods are distinguished in the following two subchapters. The first method applies for waves propagating through a constant cross-section while the second allows also for a changing cross-section and estimates the thermoacoustic functions of a whole stack or regenerator. Both methods are intended to be applied to CFD simulations.

\subsection{Thermoacoustic functions for pores with uniform cross-section}

The following method uses the temperature distribution inside the uniform pore, which is difficult to obtain in experiments, but is easy to obtain with CFD. Arnott, Bass and Raspet [52] use the relation for the cross-sectional averaged temperature for an arbitrary channel cross-section given in Equation (2.38). Assuming that there is no mean temperature gradient, Equation (2.38) simplifies to:

$$
\left\langle T_{1}\right\rangle=\frac{1}{\rho_{0} c_{p}}\left(1-f_{\kappa}\right) p_{1}
$$

This can also be written as in Hayden et al. [54]:

$$
\frac{\left\langle T_{1}\right\rangle}{T_{0}}=\frac{\gamma-1}{\gamma}\left(1-f_{\kappa}\right) \frac{p_{1}}{p_{0}}
$$

Knowing the temperature and the pressure in one cross-section from simulations or experiments, allows one to find the value of $f_{\kappa}$ for a certain frequency and geometry. To obtain the full curve of the thermoacoustic functions, the frequency in the simulations or experiments has to be varied. As in uniform pores, the viscous and the thermal thermoacoustic functions behave similar, only the behavior of the thermal thermoacoustic function has to be determined. The corresponding viscous thermoacoustic function can be deduced from the thermal one. This method can also be applied to statistically uniform materials, like random fibers, by averaging the value of the thermoacoustic functions over several cross-sections [55].

\subsection{Thermoacoustic functions for pores with non-uniform cross- section}

Another way to estimate the thermoacoustic functions of complex structures is to evaluate them globally over the whole stack or regenerator. The idea is to model the results from the numerical simulations with the one-dimensional thermoacoustic equations. This leads to a set of equations from which the thermoacoustic functions can be calculated. Equations (2.35) and (2.39) are taken as a starting point and are simplified by assuming that there is no temperature gradient. Equation (2.39) can be simplified to:

$$
d U_{1}=\frac{i \omega A d x}{\gamma p_{0}}\left[1+(\gamma-1) f_{\kappa}\right] p_{1}
$$


Using equations (2.35) and (2.47) two differential equations can be derived:

$$
\begin{aligned}
& \left(1+(\gamma-1) f_{\kappa}\right) p_{1}+\frac{c_{0}^{2}}{\omega^{2}}\left(1-f_{v}\right) \frac{d^{2} p_{1}}{d x^{2}}=0 \\
& \left(1+(\gamma-1) f_{\kappa}\right) U_{1}+\frac{c_{0}^{2}}{\omega^{2}}\left(1-f_{v}\right) \frac{d^{2} U_{1}}{d x^{2}}=0
\end{aligned}
$$

These can be solved for the pressure and the volume flow rate. Applying the boundary conditions for the pressure and the volume flow rate at position $x_{i}$, the pressure and the volume flow rate at position $x_{i+1}$ can be calculated as follows:

$$
\begin{aligned}
p_{1, i+1} & =p_{1, i} \cos \left(k \Delta x_{i, i+1}\right)-\frac{i \omega \rho_{0}}{\left(1-f_{v}\right) k} \frac{U_{1, i}}{A} \sin \left(k \Delta x_{i, i+1}\right) \\
& =p_{1, i} \cos \left(k \Delta x_{i, i+1}\right)-i Z \frac{U_{1, i}}{A} \sin \left(k \Delta x_{i, i+1}\right) \\
\frac{U_{1, i+1}}{A} & =\frac{U_{1, i}}{A} \cos \left(k \Delta x_{i, i+1}\right)-\frac{i\left(1-f_{v}\right) k}{\omega \rho_{0}} p_{1, i} \sin \left(k \Delta x_{i, i+1}\right) \\
& =\frac{U_{1, i}}{A} \cos \left(k \Delta x_{i, i+1}\right)-\frac{i}{Z} p_{1, i} \sin \left(k \Delta x_{i, i+1}\right)
\end{aligned}
$$

With the wave number $k$ defined as:

$$
k=\frac{\omega}{c_{0}} \sqrt{\frac{1+(\gamma-1) f_{\kappa}}{1-f_{v}}}
$$

and the impedance $Z$ as:

$$
Z=\frac{\rho_{0} \omega}{k\left(1-f_{v}\right)}
$$

To calculate the thermoacoustic functions, a method similar to the one from Udea et al. [56] is applied. The simulation domain is split in three parts, shown in Figure 2.8:

- $\quad$ inlet part $\left(\Delta x_{12}\right)$

- $\quad$ investigated pore $\left(\Delta x_{23}\right)$

- $\quad$ outlet part $\left(\Delta x_{34}\right)$

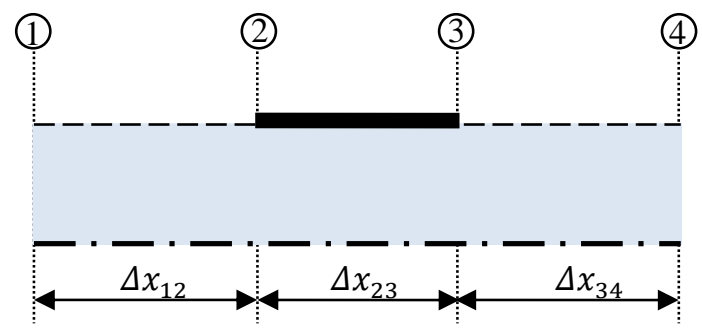

Figure 2.8: Division of the computational fluid domain into three parts to calculate the thermoacoustic functions: 1-2 inlet part; 2-3 investigated pore; 3-4 outlet part. 
Assuming that the wave propagates adiabatically in the inlet $\left(\Delta x_{12}\right)$ and outlet $\left(\Delta x_{34}\right)$ part, the corresponding thermoacoustic functions are known and the pressure and the velocity at point 2 and 3 can be calculated from Equation (2.50) and (2.51). With this, the wave number $k$ and the characteristic impedance $Z$ of the investigated pore can be calculated as follows:

$$
\begin{gathered}
k=\frac{1}{\Delta x_{23}} \arccos \left(\frac{p_{1,2} U_{1,2}+p_{1,3} U_{1,3}}{p_{1,2} U_{1,3}+p_{1,3} U_{1,2}}\right) \\
Z=A \sqrt{\frac{p_{1,3}^{2}-p_{1,2}^{2}}{U_{1,3}^{2}-U_{1,2}^{2}}}
\end{gathered}
$$

Knowing the wave number $k$ and the characteristic impedance $Z$, the thermoacoustic functions can be calculated using Equations (2.52) and (2.53).

If this method is used for short pores, the effect at the entrance of the pore becomes increasingly important as the total length becomes smaller. However, investigating short pores is of special interest to CFD, as the computational domain is smaller and thus also the computational costs. The method here corrects for the entrance effects, by comparing two simulations with different pore length. The end correction method works similar to the above described method. The only difference is that the wave number $k_{E}$ and the characteristic impedance $Z_{E}$ are not known beforehand for the entrance part $\Delta \mathrm{x}_{\mathrm{E}}$ (see Figure 2.9). Therefore, an additional simulation (B) is needed, where the inner length $\Delta x_{I}$ is varied.

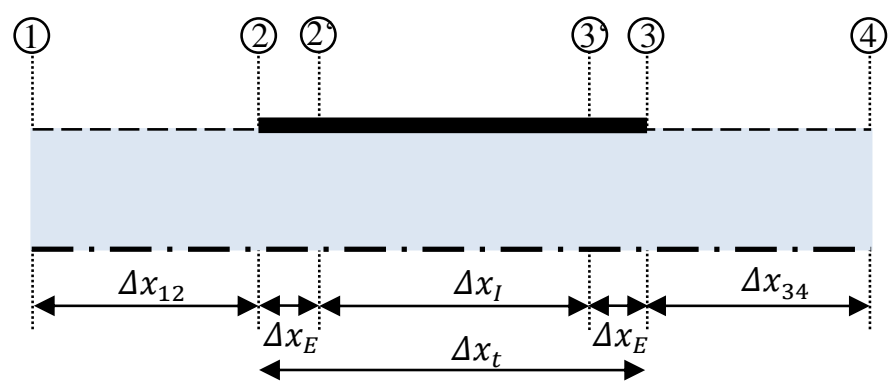

Figure 2.9: Geometric definition of the subdomains for the end-correction od: $\Delta x_{12}$ inlet length; $\Delta x_{E}$ pore end effect length; $\Delta x_{I}$ investigated inner pore length; $\Delta x_{E}$ pore end effect length; $\Delta x_{34}$ outlet length; $\Delta x_{t}$ total pore length.

The effect of the different parts adds up to the total effect of the whole pore, thus:

$$
\begin{aligned}
& 2 k_{E} \Delta x_{E}+k_{I} \Delta x_{I, A}=k_{t, A} \Delta x_{t, A} \\
& 2 k_{E} \Delta x_{E}+k_{I} \Delta x_{I, B}=k_{t, B} \Delta x_{t, B}
\end{aligned}
$$

Or: 


$$
\begin{gathered}
k_{I}=\frac{k_{t, A} \Delta x_{t, A}-k_{t, B} \Delta x_{t, 2}}{\Delta x_{t, A}-\Delta x_{t, B}} \\
k_{E}=\frac{1}{2} \frac{\Delta x_{I, B} k_{t, A} \Delta x_{t, A}-\Delta x_{I, A} k_{I} \Delta x_{I, A}}{2 \Delta x_{E}\left(\Delta x_{t, B}-\Delta x_{t, A}\right)}
\end{gathered}
$$

To calculate the characteristic impedance the same procedure can be applied and after some rearranging the impedance of the entrance area can be calculated as:

$$
Z_{E}=-\frac{1}{2} \frac{\left(-\sin \left(k_{I} \Delta x_{I, A}\right) \sin \left(k_{t, B} \Delta x_{t, B}\right) Z_{t, B}+\sin \left(k_{I} \Delta x_{I, B}\right) \sin \left(k_{t, A} \Delta x_{t, A}\right) Z_{t, A}\right)}{\cos \left(k_{E} \Delta x_{E}\right) \sin \left(k_{E} \Delta x_{E}\right)\left(\cos \left(k_{I} \Delta x_{I, B}\right) \sin \left(k_{I} \Delta x_{I, A}\right)-\cos \left(k_{I} \Delta x_{I, A}\right) \sin \left(k_{I} \Delta x_{I, B}\right)\right)}
$$

Knowing $Z_{E}$ and $k_{E}$, the same method that was described at the beginning of this chapter can be applied to get the characteristic impedance of the center part of the pore.

\subsection{Low reduced frequency approximations}

In this chapter the linearized Navier-Stokes equations are solved for three specific geometries without a temperature gradient. The flow field is derived and used in Chapter 4 to validate the CFD simulations and select the adequate discretization parameters. The following derivations closely follow those found in [57], [58] and [59]. First in Chapter 2.3.1 the general solution method is presented, then this method is applied to obtain the solution of the flow field in a straight tube with circular cross-section (Chapter 2.3.2), a vibrating resonator (Chapter 2.3.3) and finally in a bent tube (Chapter 2.3.4).

\subsubsection{General solution method}

The starting point of the derivation are the linearized Navier-Stokes equations as derived in Chapter 2.2.2. These consist of the conservation equations for mass (2.25), momentum (2.26) and energy (2.27) and are closed by the ideal gas law (2.28).

The idea for solving these equations is to decouple the governing equations such that the contributions in the cross-direction can be separated from the contributions in the wave propagation direction. In order to split the equations into the different contributions, the gradient is split into its contribution in the cross-direction $(c d)$ and the propagation direction $(p d)$ :

$$
\nabla=\nabla_{c d}+\nabla_{p d}
$$

Applying the following additional assumptions, the governing equations can be simplified [59]:

- the viscous and thermal boundary layer are small compared to the wavelength

- the hydraulic radius is small compared to the acoustic wavelength

- the hydraulic radius is small compared to the length of the waveguide

- no mean temperature gradient exists

From these assumptions it follows that the magnitude of the velocities in the crossdirection are much smaller than the magnitude of the velocities in propagation direction. Furthermore, the gradient of the velocity in the propagation direction $v_{p d}$ and the gradi- 
ent of the temperature are much larger in the cross-direction compared to the propagation direction. Finally, the conservation equations can be written as:

$$
\begin{gathered}
i \omega \rho_{1}+\nabla_{\mathrm{cd}} \cdot\left(\rho_{0} v_{c d}\right)+\nabla_{p d} \cdot\left(\rho_{0} v_{p d}\right)=0 \\
i \omega \rho_{0} v_{p d}=-\nabla_{\mathrm{pd}} p_{1}+\mu \Delta_{\mathrm{cd}} v_{p d} \\
0=\nabla_{\mathrm{cd}} p_{1} \\
c_{p} \rho_{0} i \omega T_{1}=k \Delta_{\mathrm{cd}} \mathrm{T}_{1}+i \omega p_{1} \\
\frac{p_{1}}{p_{0}}=\frac{\rho_{1}}{\rho_{0}}+\frac{T_{1}}{T_{0}}
\end{gathered}
$$

This is the set of equations from which the low reduced frequency models are derived. This set of equations can be solved for different geometries by choosing the appropriate coordinate system and the right boundary conditions. In all cases, equation (2.63) and (2.65) can be solved for the temperature and the velocity in wave propagation direction, the solutions have the general form:

$$
\begin{aligned}
T_{1} & =\frac{1}{c_{p} \rho_{0}}\left(1-h_{\kappa}\right) p_{1}+h_{\kappa, I H} \\
v_{p d} & =\frac{i}{\omega \rho_{0}}\left(1-h_{v}\right) \nabla_{p d} p_{1}+h_{v, I H}
\end{aligned}
$$

The functions $\left(1-h_{\kappa}\right)$ and $\left(1-h_{v}\right)$ are the particular solution of equations (2.63) and (2.65) with homogeneous boundary conditions, while the functions $h_{\kappa, I H}$ and $h_{v, I H}$ are the solutions that satisfy the inhomogeneous boundary conditions. They are dependent on the cross-section coordinates. In the case of a straight tube with circular crosssection (Chapter 2.3.2) and the bend tube (Chapter 2.3.4) the wall velocity and oscillating temperature are zero. Thus, $h_{\kappa, B C}$ and $h_{v, I H}$ are zero. For the vibrating resonator (Chapter 2.3.3) the walls are moving and the term $h_{v, I H}$ is non-zero, while $h_{\kappa, I H}$ is zero. The function $h_{\kappa, I H}$ does not appear in any of the cases discussed in this thesis. For this reason it will be dropped in the following derivation. For a more complete derivation see [59].

Equation (2.67) and (2.68) can be related to the thermoacoustic equations obtained in Chapter 2.2.3 by spatially averaging them, as the spatial average of the functions $h_{\kappa}$ and $h_{v}$ yield the thermoacoustic functions $f_{\kappa}$ and $f_{v}$ (see Chapter 2.2.4).

The density can be obtained by inserting the temperature (2.67) in the equation of state (2.66):

$$
\rho_{1}=\frac{p_{1}}{p_{0}} \rho_{0}\left(1-\frac{\gamma-1}{\gamma}\left(1-h_{\kappa}\right)\right)
$$

The equation to calculate the pressure is derived by integrating the velocity in the propagation direction (2.68) and the density (2.69) over the cross-section and inserting them into the cross-sectionally averaged continuity equation (2.62). Assuming that the wall does not move in the cross-direction and has a constant cross-section in the wave propagation direction, the differential equation for the pressure is obtained: 


$$
\frac{\omega^{2}}{c_{0}^{2}} \Gamma^{2} p_{1}+\Delta_{p d} p_{1}=0
$$

with the propagation constant $\Gamma$ defined as:

$$
\Gamma=\sqrt{\frac{\gamma}{n\left(1-f_{v}\right)}}
$$

and the kind of polytropic constant $n$ :

$$
n=\left(1-\frac{\gamma-1}{\gamma}\left(1-f_{\kappa}\right)\right)^{-1}
$$

In the following chapters equation (2.70), (2.67), (2.68) and (2.69) can be solved to obtain the flow field for different geometries and boundary conditions.

\subsubsection{Straight tube with circular cross-section}

The first solution presented here is the acoustic wave propagation in a circular tube. Solutions for this geometry were reviewed by [57]. A cylindrical coordinate system is chosen, in which the wave propagates in the direction of the $z$-axis. In this coordinate system the problem can be reduced to a two-dimensional problem where the gradient and Laplace operator are given by:

$$
\begin{gathered}
\nabla_{p d}=\frac{\partial}{\partial z} \vec{e}_{z} \\
\nabla_{c d}=\frac{\partial}{\partial r} \vec{e}_{r} \\
\Delta_{c d}=\frac{1}{r} \frac{\partial}{\partial r}+\frac{\partial^{2}}{\partial r^{2}}
\end{gathered}
$$

To solve the problem the following boundary conditions are needed:

$$
\begin{aligned}
& u_{1}(z, R)=0 \\
& T_{1}(z, R)=0
\end{aligned}
$$

Introducing these boundary conditions into equations (2.63) and (2.65) the solution for the functions $h_{v}$ and $h_{\kappa}$ is given by:

$$
\begin{gathered}
h_{v}=\frac{J_{0}\left((i-1) \frac{r}{\delta_{v}}\right)}{J_{0}\left((i-1) \frac{R}{\delta_{v}}\right)}=\frac{J_{0}\left(i^{\frac{3}{2}} \eta s\right)}{J_{0}\left(i^{\frac{3}{2}} s\right)} \\
h_{\kappa}=\frac{J_{0}\left((i-1) \frac{r}{\delta_{\kappa}}\right)}{J_{0}\left((i-1) \frac{R}{\delta_{\kappa}}\right)}=\frac{J_{0}\left(i^{\frac{3}{2}} \eta s P r\right)}{J_{0}\left(i^{\frac{3}{2}} s P r\right)}=\frac{J_{0}\left(i^{\frac{3}{2}} \eta s_{t}\right)}{J_{0}\left(i^{\frac{3}{2}} s_{t}\right)} \\
h_{\kappa, I H}=h_{v, I H}=0
\end{gathered}
$$

The velocity, temperature and density can be calculated according to equations (2.67), (2.68) and (2.69). The pressure is given by: 


$$
\begin{aligned}
p_{1} & =A \exp \left(\Gamma \frac{\omega}{c_{0}} z\right)+B \exp \left(-\Gamma \frac{\omega}{c_{0}} z\right) \\
& =A \exp (\Gamma \xi)+B \exp (-\Gamma \xi)
\end{aligned}
$$

with the wave propagation constant $\Gamma$ calculated according to (2.71) and (2.72). The integration constants $A$ and $B$ can be obtained by imposing boundary conditions in the axial direction.

From the dimensionless equations of the flow variables it can be seen that the shear wave number is the governing dimensionless number, pertaining to the velocity profile over the dimensionless radius. Figure 2.10 shows the dimensionless velocity profiles for different shear wave numbers over the dimensionless radius. For higher shear wave numbers the inertial effects are more important than the viscous effects. This results in nearly constant values in the center of the tube, while at the wall a clear boundary layer is visible. For lower shear wave numbers, the viscous effects dominate and the boundary layer fills the whole radius.

Another interesting flow effect is the overshooting at the boundary. This effect is caused by the interaction between the pressure and the velocity (or temperature) at a wall. Assuming that the velocity reached its maximum, a profile similar to the one with $s=20$ in Figure 2.10 can be observed. The velocity close to the wall at this moment in time is smaller than in the center of the tube. In the following half period the bulk fluid is accelerated in the negative direction. As the velocity close to the wall was already smaller than in the center, it reaches a lower negative value than in the center and a velocity overshoot occurs.

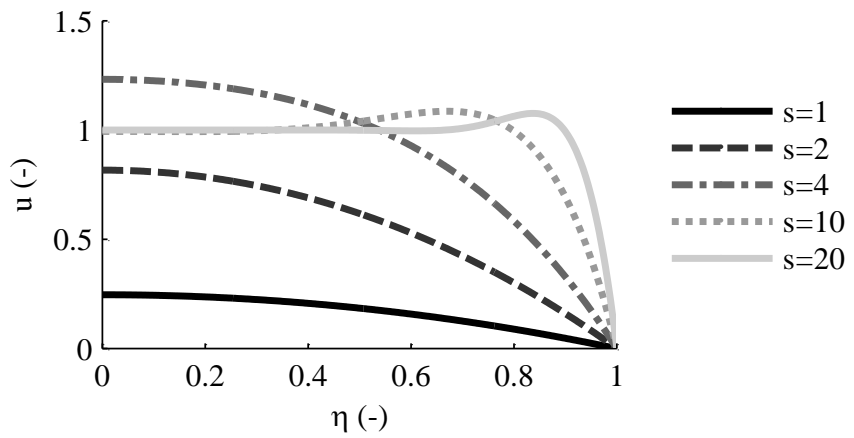

Figure 2.10: Velocity profiles for five shear wave numbers.

\subsubsection{Vibrating resonator}

In this chapter the solution for a vibrating resonator is presented. It is a twodimensional test case, which does not incorporate sophisticated boundary conditions in CFD. For this reason it will be used to choose the right discretization of the boundary layers in Chapter 4.1. The same coordinate system as for the cylindrical tube in Chapter 2.3.2 is chosen, only the boundary conditions change to: 


$$
\begin{gathered}
u_{1}(z, R)=u_{w} \cos (\omega t) \\
T_{1}(z, R)=0
\end{gathered}
$$

Introducing these boundary conditions into equations (2.63) and (2.65) the solutions to the function $h$ are given by:

$$
\begin{gathered}
h_{v}=\frac{J_{0}\left((i-1) \frac{r}{\delta_{v}}\right)}{J_{0}\left((i-1) \frac{R}{\delta_{v}}\right)}=\frac{J_{0}\left(i^{\frac{3}{2}} \eta s\right)}{J_{0}\left(i^{\frac{3}{2}} s\right)} \\
h_{\kappa}=\frac{J_{0}\left((i-1) \frac{r}{\delta_{\kappa}}\right)}{J_{0}\left((i-1) \frac{R}{\delta_{\kappa}}\right)}=\frac{J_{0}\left(i^{\frac{3}{2}} \eta s P r\right)}{J_{0}\left(i^{\frac{3}{2}} s P r\right)}=\frac{J_{0}\left(i^{\frac{3}{2}} \eta s_{t}\right)}{J_{0}\left(i^{\frac{3}{2}} s_{t}\right)} \\
h_{v, I H}=u_{w} \cdot \frac{J_{0}\left(i^{\frac{3}{2}} \eta s\right)}{J_{0}\left(i^{\frac{3}{2}} s\right)}
\end{gathered}
$$

The solution for the pressure is the same as the one presented in Chapter 2.3.2:

$$
\begin{aligned}
p_{1} & =A \exp \left(\Gamma \frac{\omega}{c_{0}} z\right)+B \exp \left(-\Gamma \frac{\omega}{c_{0}} z\right) \\
& =A \exp (\Gamma \xi)+B \exp (-\Gamma \xi)
\end{aligned}
$$

The constants $A$ and $B$ can be obtained for a closed geometry of length $L$ by imposing the velocity at the axial ends of the resonator:

$$
\begin{aligned}
& u_{1}(0, r)=0 \\
& u_{1}(L, r)=0
\end{aligned}
$$

The flow field is qualitatively the same as for a cylindrical tube. The only change is that there is an additional term for the axial velocity due to the moving wall.

\subsubsection{Curved layer}

The last low reduced frequency solution discussed here, is the solution for the flow in a curved layer. The solution presented here is a special case of the general equations derived by Nijhof [59]. The solution is used to validate the simulation of losses in a bend. It is valid for low curvatures and is only two-dimensional, which is favorable for CFD simulations. In case of large curvatures the low reduced frequency solution no longer holds and CFD simulations are necessary to reveal the flow field.

The curved layer is defined by the curvature $1 / R$ and the half spacing $h$ (see Figure 2.11). A cylindrical coordinate system is chosen, but the wave does not propagate in the axial direction, but in the angular direction, $\varphi$. The gradients in the cross-direction and propagation direction can be written as:

$$
\nabla_{p d}=\frac{1}{r} \frac{\partial}{\partial \varphi} \vec{e}_{\theta}
$$




$$
\begin{gathered}
\nabla_{c d}=\frac{\partial}{\partial r} \vec{e}_{r} \\
\Delta_{c d}=\frac{1}{r} \frac{\partial}{\partial r}+\frac{\partial^{2}}{\partial r^{2}}-\frac{1}{r^{2}}
\end{gathered}
$$

Using the following boundary conditions at the walls:

$$
\begin{aligned}
& u_{1}(R \pm h)=0 \\
& T_{1}(R \pm h)=0
\end{aligned}
$$

analytical solutions for the velocity, temperature and the density can be obtained. Although analytical solutions exist, these solutions are cumbersome. Hence, these solutions are not given here. Instead, the velocity in the wave propagation direction and the temperature profile are plotted. Figure 2.12 shows the velocity a) and temperature profile b) for a constant radius $\mathrm{R}$, at three different values of the half spacing $h$. For smaller half spacing $h$ and thus smaller shear wave numbers $s$, the boundary effects extend over the whole height of the curved layer. For larger values of the shear wave number a clear boundary layer close to the walls can be distinguished from an inner part. The inner profile of the temperature is constant over the radius $r$, while the inner part of the velocity decreases towards higher radii $r$. This is due to the fact, that the temperature is driven by the pressure (see equation (2.65)), while the velocity is driven by the angular gradient of the pressure (see equation (2.63)). As the angular gradient is larger for smaller radii $r$, the velocity is larger in this part close to the inner wall. This is illustrated in Figure 2.13 which shows the velocity a) and angular momentum profile b) for different radii $R$, but constant half spacing $h$. For sharp curved layers, the velocity is high for small radii $r$, but the angular momentum is the same for all three geometries. This is due to the assumption that the pressure is constant over the cross-section, which does not hold for very sharp curved layers. This is the motivation for using CFD simulation to reveal the flow field even for sharp curves.

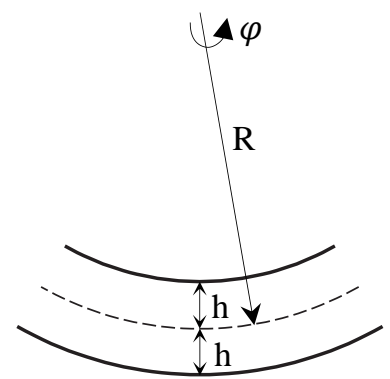

Figure 2.11: Dimensions of the curved layer; radius $R$ and half spacing $h$. 


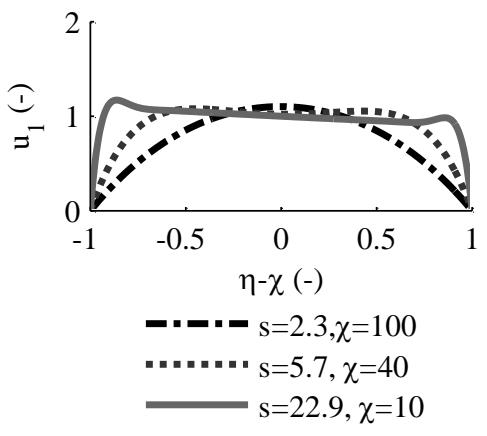

a)

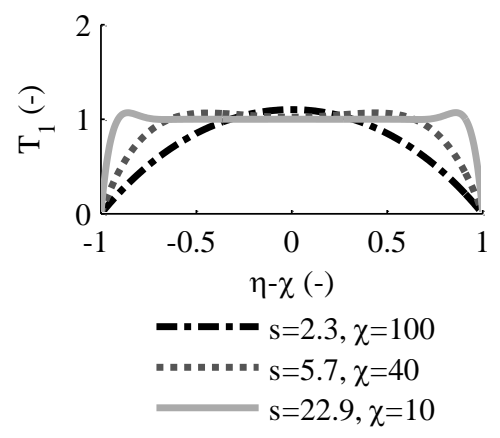

b)

Figure 2.12: a) Velocity in wave propagation direction and b) temperature profile over the dimensionless radial component $\eta$ for four different heights $h$.

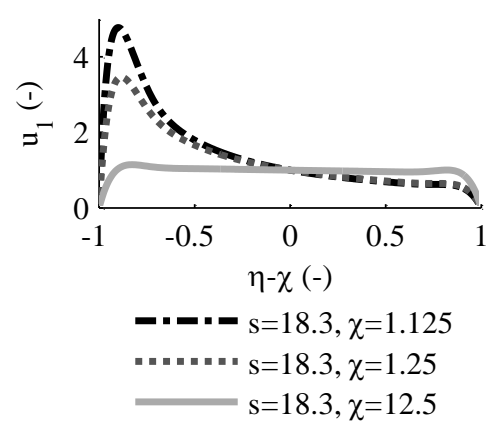

a)

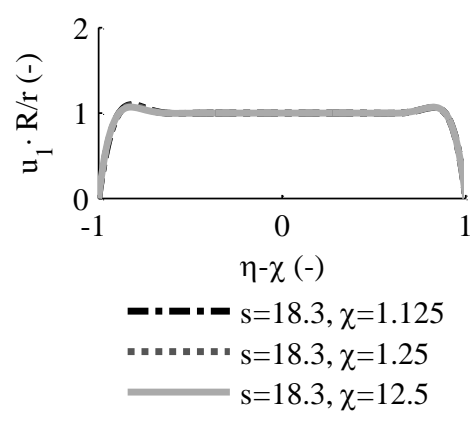

b)

Figure 2.13: Profile of the velocity in wave propagation direction a) and the angular momentum b) for three different radii $R$ and constant half spacing $h$.

In Chapter 4 the CFD simulations will be validated with the low reduced frequency approximations presented in this chapter. In Chapters 5 the CFD simulations will be applied to cases where the low reduced frequency approximations are no longer valid. 


\section{Computational fluid dynamics}

The Navier Stokes equations do not have a unique solution and only in some simple cases an analytical solution can be derived. In some other cases assumptions can be made which provide a solution to the Navier Stokes equations for a specific case, as was shown in Chapter 2 for the thermoacoustic equations. But these equations are only valid when three-dimensional effects, like for example vortex shedding, are negligible. To resolve these three-dimensional effects, the Navier Stokes equations have to be solved numerically. Well-established techniques such as the Finite Difference method, the Finite Volume method and the Finite Element method [60] can be used. For this work the commercially available CFD software ANSYS Fluent [39] was chosen and is presented in Chapter 3.1.

Even though CFD can be applied in a variety of applications, it is necessary for each of these applications to conduct a verification and validation of the code and the numerical parameters as defined by [61]. Because in this work commercially available CFD software is used, no verification of the code is possible. A validation of numerical simulations in thermoacoustics is done in Chapter 4.1 and a choice of numerical parameters is presented. The non-reflecting and impedance boundary conditions are presented in Chapter 3.2 and verified and validated in Chapters 4.2 through 4.4. This results in reliable CFD simulations of thermoacoustic components.

\subsection{Navier-Stokes equations}

The commercial CFD software used in this research is ANSYS Fluent 14.0 [39]. It solves the three-dimensional time dependent compressible conservation equation as shown below:

Mass conservation:

$$
\frac{\delta \rho}{\delta t}+\nabla \cdot(\rho \vec{v})=S_{m}
$$

Momentum conservation:

$$
\frac{\delta}{\delta t}(\rho \vec{v})+\nabla \cdot(\rho \vec{v} \vec{v})=-\nabla p+\nabla \cdot(\overline{\bar{\tau}})+\rho \vec{g}+\vec{F}
$$

Energy conservation:

$$
\frac{\delta}{\delta t}(\rho e)+\nabla \cdot(\vec{v}(\rho e+p))=\nabla \cdot(k \nabla T+(\overline{\bar{\tau}} \cdot \vec{v}))+S_{h}
$$

where $S_{m}, \vec{F}, S_{h}$ are source terms in the respective conservation equation and with $\overline{\bar{\tau}}$ the stress tensor:

$$
\overline{\bar{\tau}}=\mu\left(\left(\nabla \vec{v}+\nabla \vec{v}^{T}\right)-\frac{2}{3} \nabla \cdot \vec{v} I\right)
$$

The specific energy $e$ is defined as: 


$$
e=h-\frac{p}{\rho}+\frac{\vec{v}^{2}}{2}
$$

To close the system of equations an equation of state is needed. As the working fluid is helium, assuming that it behaves like an ideal gas is a good approximation:

$$
\rho=\frac{p}{R_{s} T}
$$

No turbulence model is used, as the implemented models in ANSYS Fluent have low pressure amplitudes.

The conservation equations are solved using the finite volume method [60]. The geometry is divided into a finite number of volumes, for which the conservation equations are solved. The flow variables are calculated at the center of each volume. In order to obtain the values on the surface of every volume an interpolation is done between the center values of neighboring cells ${ }^{1}$. Gradients at the surfaces are estimated from the neighboring cells with the discretization schemes ${ }^{2}$.

The pressure-based solver is used to solve the conservation equation, as it was developed for low-speed flows [62]. Furthermore, most of the reported thermoacoustic CFD simulations conducted with ANSYS Fluent are done with the pressure based solver. Some examples are: $[40,63,64,65,66,67]$. The solver calculates the velocity field from the momentum equation and the pressure from a pressure correction equation, which is obtained by manipulating the continuity and the momentum equation [62].

The spatial and temporal discretizations are discussed in more details in Chapter 4, where they are investigated for some test cases. In order to solve the equations given above, boundary conditions are needed. These are presented in the following chapter.

\subsection{Boundary conditions}

The Navier-Stokes equations are valid for a large amount of flows. To investigate specific flow situations and regimes, the correct boundary conditions have to be chosen. A common choice in CFD is to apply the velocity and the temperature at the inlet of the domain and the pressure at the outlet of the domain.

However in (thermo)acoustic simulations the flow is oscillating, which makes the choice of the boundary condition more challenging. Applying an oscillating boundary condition at the inlet, with a non-matching boundary condition at the outlet would lead to unwanted reflections. Non-reflecting and impedance boundary conditions are needed for (thermo)acoustic simulations in order to allow the acoustic waves to leave the domain without causing unwanted reflections. As no impedance boundary conditions are imple-

${ }^{1}$ The different interpolation schemes implemented in ANSYS Fluent are presented in Chapter 4.2.2.1.

${ }^{2}$ The different discretization schemes implemented in ANSYS Fluent are presented in Chapter 4.2.2.2. 
mented in ANSYS Fluent, both a non-reflecting and an impedance boundary condition where implemented with the use of User Defined Functions (UDF). The underlying idea of the non-reflecting boundary condition and the impedance boundary condition are introduced in Chapter 3.2.1 and 3.2.2, respectively. In Chapter 3.2.4 the implementation in ANSYS Fluent is described.

\subsubsection{Non-reflecting boundary condition}

Nonreflecting boundary conditions are of great importance for thermoacoustic simulations to reduce the computational domain and with this the computational costs. While it is easy in acoustic models to impose the wave entering the domain, this is much more complex in CFD, as the total value of the pressure and the velocity have to be imposed at the boundaries. In order to obtain a boundary condition which does not reflect the wave in an undesirable way, the incoming and outgoing waves have to be known at the boundaries.

The idea behind the non-reflecting boundary is similar to the one presented in [68] and uses the most general solution of the one-dimensional wave equation for the pressure $p$ and the velocity $u[50]$ :

$$
\begin{gathered}
p=f\left(t-\frac{x}{c_{0}}\right)+g\left(t+\frac{x}{c_{0}}\right) \\
u=\frac{1}{\rho_{0} c_{0}}\left(f\left(t-\frac{x}{c_{0}}\right)-g\left(t+\frac{x}{c_{0}}\right)\right)
\end{gathered}
$$

In equation (3.7) and (3.8), $f\left(t-x / c_{0}\right)$ represents the forward traveling wave, while $g\left(t+x / c_{0}\right)$ represents the backward traveling wave. Assuming no mean flow, the pressure and velocity can be calculated as a superposition of these two waves. At a boundary as shown in Figure 3.1 the incoming wave $f$ (blue) shall be specified, while the outgoing wave $g$ (green) is given by the fluid flow inside the domain.

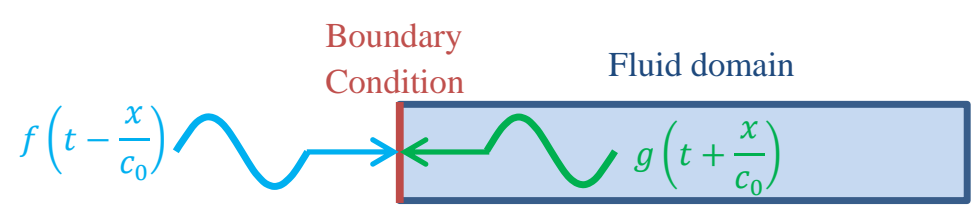

Figure 3.1: Forward and backward traveling wave at the domain boundary condition ("left boundary").

In order to apply the correct pressure at the boundary $x_{B}$, the value of $g$ has to be known. To calculate the wave leaving the domain at the time $t$, the propagation characteristics of acoustic waves are used. The following equality, which is graphically shown in Figure 3.2, is valid:

$$
g\left(t+\frac{x_{b}}{c_{0}}\right)=g\left(t-\Delta t+\frac{x_{b}}{c_{0}}+\frac{\Delta x}{c_{0}}\right)
$$


This equality is valid if the wave propagates within the time $\Delta t$ by a distance $\Delta x$ without damping:

$$
\Delta x=c_{0} \Delta \mathrm{t}
$$

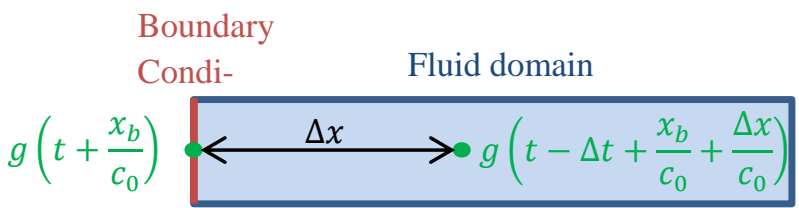

Figure 3.2: Definition of the monitor point for computing the backward traveling wave at the boundary.

In the following subsections two different boundary conditions will be presented, the first assumes ideal one-dimensional wave propagation and is presented in Chapter 3.2.1.1. The second extends this idea to general plane waves with velocity profiles in the direction orthogonal to the wave propagation direction. This boundary condition is presented in Chapter 3.2.1.2. In Chapter 3.2.1.3 it is shown how non ideal wave propagation and damping can be taken into account.

\subsubsection{One-dimensional wave propagation}

In a first approach, lossless acoustics are assumed. This means that the wave is onedimensional and no changes are to be expected in the direction orthogonal to the wave propagation direction. The procedure is derived for a boundary condition at the left side of the domain as shown in Figure 3.1. The procedure for a boundary at the right side of a domain can be derived in the same way. The wave $g$ leaving the domain at time $t$ can be calculated by sampling the pressure and velocity at the location $x_{b}+\Delta x$ at the previous time $t-\Delta t$. The pressure and the velocity is monitored at this location and with equations (3.7) and (3.8) the backward traveling wave can be calculated as:

$$
g\left(t+\frac{x_{b}}{c_{0}}\right)=\frac{1}{2}\left(p\left(t-\Delta t, x_{b}+\Delta x\right)-\rho_{0} c_{0} u\left(t-\Delta t, x_{b}+\Delta x\right)\right)
$$

Knowing the backward traveling wave at the monitor point and the fact that it travels to the boundary within one time step, the boundary condition can be imposed by superposition of the imposed forward traveling wave $f_{B}(t)$ and the calculated backward traveling wave. The whole procedure for a boundary condition at the left of a domain assuming lossless acoustics is summarized in Figure 3.3. On the horizontal axis the location of the boundary $x_{B}$ and the monitor point $x_{M}$ are shown, while the vertical axis represents a time axis with two time steps $t$ and $t-\Delta t$. The information about the backward traveling wave $g$ is calculated from the monitor point $x_{M}$ at the time step $t-\Delta t$. Then the wave travels to the boundary within the time step $\Delta t$. The backward traveling wave is thus known at the boundary $x_{B}$ at the time $\mathrm{t}$ and the pressure boundary condition $p$ can be imposed. 


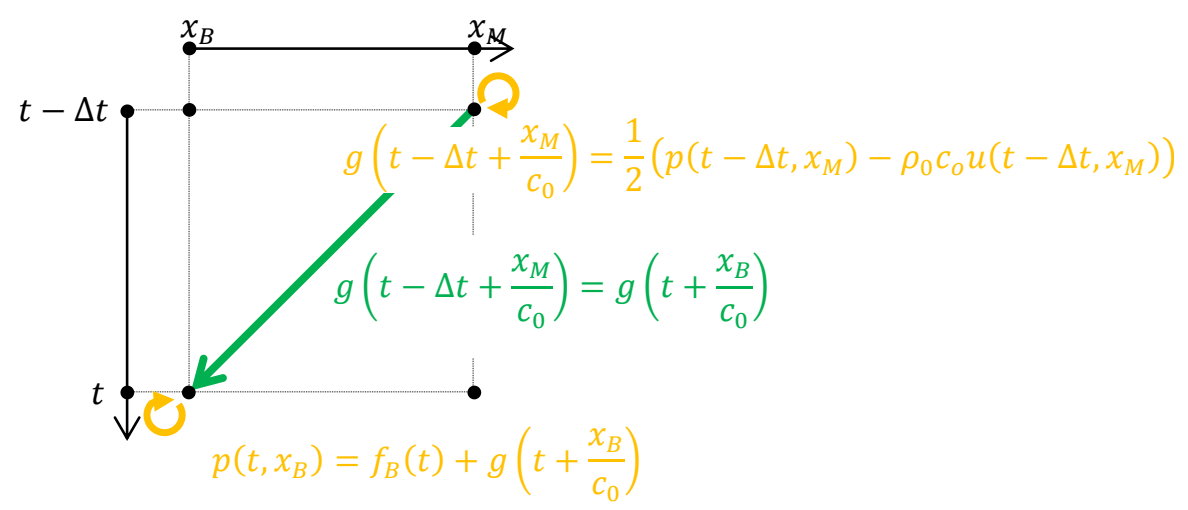

Figure 3.3: Functioning of the non-reflecting boundary condition without boundary layer effects.

\subsubsection{Wave propagation in a pipe}

In cases where a velocity boundary layer occurs it is not possible to calculate the backward traveling wave from the pressure and the velocity at the monitor point, as shown in equation (3.11). But when the radius is small compared to the wavelength, the pressure is nearly uniform in the direction orthogonal to the wave propagation, even though the velocity shows a non-uniform profile. The underlying assumption is that the reduced frequency is low $k=\omega R / c_{0} \ll 1$, which is usually the case in thermoacoustic devices. Using this assumption it is possible to derive a more general form of the nonreflecting boundary condition.

In this case we use the fact that the pressure at the monitor point is a superposition of the forward and backward traveling wave (Equation (3.7)). Applying this idea equation (3.11) can be replaced by

$$
g\left(t+\frac{x_{B}}{c_{0}}\right)=p\left(t-\Delta t, x_{b}+\Delta x\right)-f\left(t-\Delta t-\frac{x_{b}+\Delta x}{c_{0}}\right)
$$

such that no velocity information is required. The forward traveling wave is given at the boundary one time step before reaching the monitor point (using Equation (3.10)). Thus, a further time step back in time is needed to calculate the pressure boundary condition at the time $t$. Different ways to model the wave propagation and to account for dissipation and dispersion due to the viscous effects are presented in the Chapter 3.2.1.3. The procedure for general plane wave propagation is summarized in Figure 3.4. The horizontal axis shows the location of the boundary $x_{B}$ and of the monitor point $x_{M}$. The vertical axis shows the three time steps necessary to calculate the pressure at the boundary $p$ at the time $t$. 


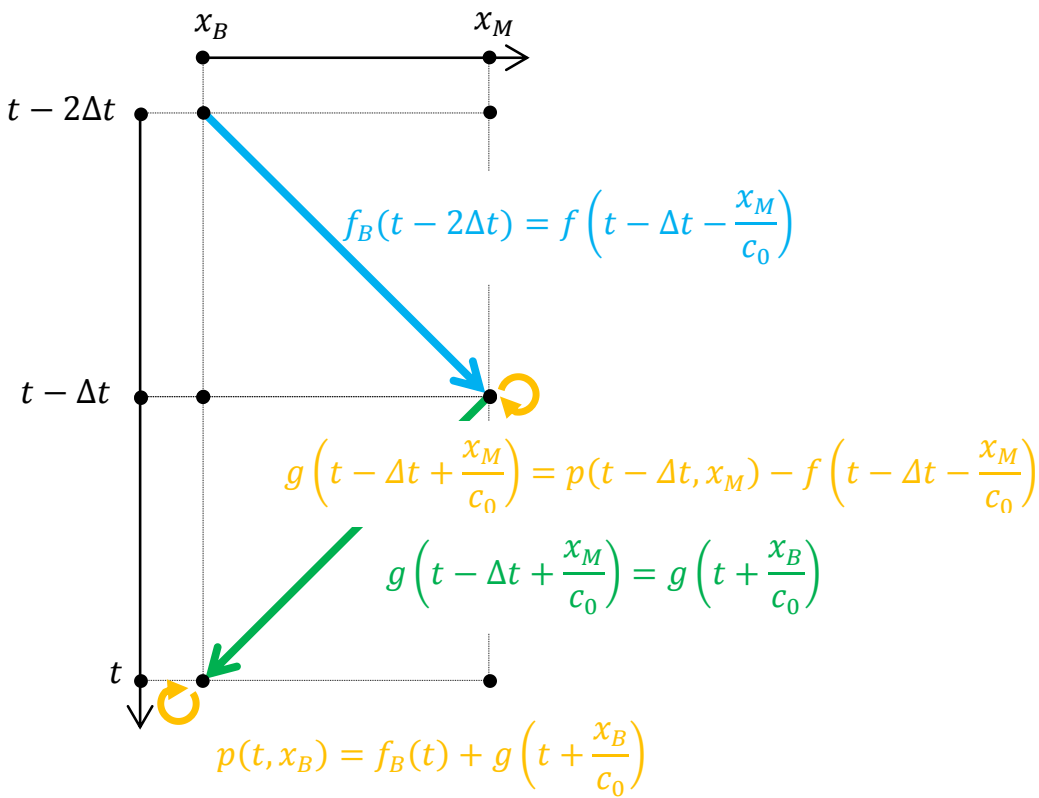

Figure 3.4: Functioning of the non-reflecting boundary condition with boundary effects.

\subsubsection{Extension of the non-reflecting boundary condition for non-ideal wave propagation}

For high pressure amplitudes or small diameters, the wave does not travel ideally as assumed in equation (3.9). Dissipation and changes in the wave propagation speed need to be taken into account. In this chapter three different ways to account for these effects are presented.

\subsection{Inclusion of dissipative effects in small channels}

If waves propagate in small tubes, the dissipative effects cannot be neglected between the boundary and the monitor point, as this would create unphysical reflections. These effects can be taken into account by means of the complex wave propagation constant $\Gamma$. This can be determined, for example, from the low reduced frequency model [57], the thermoacoustic functions [52] or from other simulations. Thus, equation (3.9) has to be adapted such that it accounts for dissipative effects as follows:

$$
g\left(t+\frac{x_{b}}{c_{0}}+\frac{\Delta x}{c_{0}}\right)=g\left(t+\Delta t+\frac{x_{b} \operatorname{Im}(\Gamma)}{c_{0}}\right) \cdot \exp (\operatorname{Re}(\Gamma) \cdot \Delta x)
$$


The real part of the propagation constant induces a damping of the amplitude while the imaginary part reduces the propagation speed of the wave and with this the position of the monitor point.

As will be mentioned later in Chapter 3.2.3, the temperature profile during the half period of inflow is computed from the pressure with the assumption of adiabatic wave propagation. This means that a constant temperature over the height is assumed. When dissipative effects due to a nearby thermally interacting wall are of importance, this no longer holds. Figure 3.5 shows the temperature profile in a case, where the wave propagates between two isothermal parallel plates with four different shear wave numbers. The temperature distribution shows a non-uniform profile over the height.

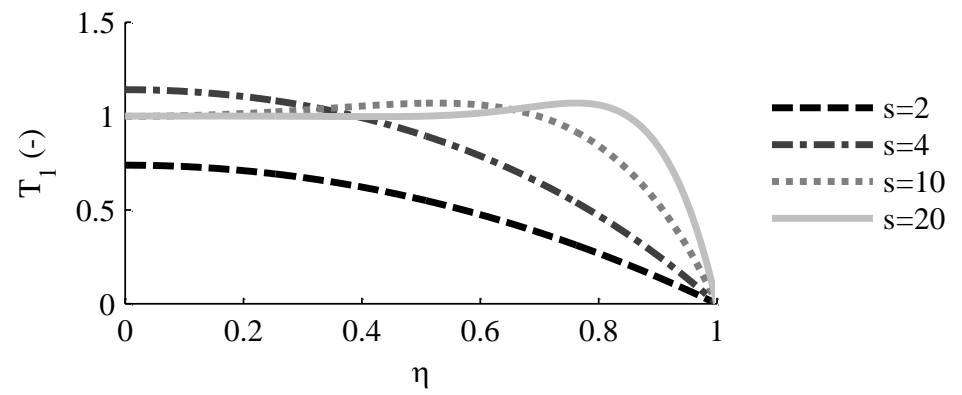

Figure 3.5: Normalized temperature profile between two isothermal parallel plates for four different shear wave number over the dimensionless half spacing $\eta$.

If an analytical solution is known, for example for parallel plates as shown in Figure 3.5, the solution can be used to impose the temperature profile from the pressure. Otherwise one has to be aware that the boundary condition introduces a thermal error during the inflow into the domain.

\subsection{Inclusion of nonlinear effects at high amplitudes}

Nonlinear effects become more and more important with increasing pressure amplitude. In Blackstock [50] the general solution for the nonlinear wave equation for a plane progressive wave traveling in the positive $x$-direction is derived as:

$$
p=f\left(t-\frac{x}{c_{0}+\beta u}\right)
$$

where $\beta$ is the coefficient of nonlinearity, defined for ideal gases as:

$$
\beta=\frac{1}{2}(\gamma+1)
$$

Comparing Equation (3.7) and (3.14) shows that the unperturbed wave propagation speed, $c_{0}$, for linear acoustics (3.7) is replaced by $c_{0}+\beta u$ in the nonlinear case (3.14). Even though the difference between these two wave propagation speeds is small, the effect is cumulative and leads to wave steepening. At the boundary an accumulating error 
could lead to a drift of the boundary values. Rewriting the wave propagation speed as [50]:

$$
c_{0}+\beta u=c+u
$$

shows that there are two effects causing nonlinear wave propagation. The first term on the right side shows that the wave propagation speed is not constant but varies with the temperature amplitude as $c \propto \sqrt{T}$. The second term on the right hand side, $u$, introduces convection of the sound wave. To account for nonlinearities at high amplitudes in the boundary condition, the wave propagation speed $c_{0}$ is replaced by $c_{0}+\beta u$ in case of a forward traveling wave and $c_{0}-\beta u$ for a backward traveling wave:

$$
\begin{aligned}
& f\left(t+\Delta t-\frac{x_{b}}{c_{0}+\beta u}\right)=f\left(t-\frac{x_{b}}{c_{0}+\beta u}-\frac{\Delta x}{c_{0}+\beta u}\right) \\
& g\left(t+\Delta t+\frac{x_{b}}{c_{0}-\beta u}\right)=g\left(t+\frac{x_{b}}{c_{0}-\beta u}+\frac{\Delta x}{c_{0}-\beta u}\right)
\end{aligned}
$$

Thus, the forward traveling wave travels

$$
\Delta x=\Delta t\left(c_{0}+\beta u\right)
$$

within one time step and the backward traveling wave

$$
\Delta x=\Delta t\left(c_{0}-\beta u\right)
$$

\subsection{Plane wave averaging}

In thermoacoustic simulations where plane wave propagation can be assumed, this property can be used to make the non-reflecting boundary conditions more stable. Oscillating behavior on the boundary can be avoided by averaging over the direction orthogonal to the wave propagation direction. Polifke et al. [69] separate the pressure into three different components, the mean pressure $p_{0}$, the pressure fluctuation $p_{1}$ and the turbulent pressure component $p^{\prime}$.Here we do not account for turbulent fluctuations and use $p^{\prime}$ for numerical noise:

$$
p(x, t)=p_{0}(x)+p_{1}(x, t)+p^{\prime}(x, t)
$$

The same separation is applied to the velocity:

$$
u(x, t)=u_{0}(x)+u_{1}(x, t)+u^{\prime}(x, t)
$$

Taking the area averaged $\langle.$.$\rangle over the plane orthogonal to the wave propagation di-$ rection and assuming that the length scale of the numerical noise is small enough, the numerical noise almost vanishes:

$$
\begin{aligned}
& \left\langle p^{\prime}\right\rangle \approx 0 \\
& \left\langle u^{\prime}\right\rangle \approx 0
\end{aligned}
$$

If no numerical noise occurs, the pressure and velocity are constant in the direction orthogonal to the wave. Thus, averaging the left and right going waves over the orthogonal direction should reduce numerical errors. Another advantage of the averaging is that only one value has to be saved for the next time step, instead of every grid point in the orthogonal direction. 


\subsubsection{Impedance boundary condition}

An acoustic domain is often reduced by imposing an acoustic impedance boundary condition, which models the domain that is not taken into account. In CFD the impedance boundary conditions are more difficult to implement as the CFD simulations take place in the time domain and not in the frequency domain. In the following, an impedance boundary condition is proposed which is applicable if the driving frequency is known beforehand, which is generally the case for thermoacoustic CFD simulations.

The pressure and velocity can be written in terms of left and right traveling waves like in Equation (3.7) and (3.8). In complex notation this can be written as:

$$
\begin{aligned}
& p=p_{+} e^{i \alpha+i \omega t}+p_{-} e^{i \beta+i \omega t} \\
& u=u_{+} e^{i \alpha+i \omega t}-u_{-} e^{i \beta+i \omega t}
\end{aligned}
$$

where the plus ("+") indicates a forward and the minus ("-") a backward traveling wave. The angles $\alpha$ and $\beta$ are phase delays. As we are only interested in the phase difference between the incoming and the outgoing wave, one of both angles can be chosen freely. Assuming that the wave is leaving at a boundary at the right of the computational domain, the outgoing, forward traveling wave is given by the flow field in the domain, while the backward traveling wave has to be calculated to impose the correct boundary condition. Therefore, $\alpha$ is chosen as a reference and set to zero. Using this reference the acoustic impedance at the right boundary can be written as:

$$
Z_{a c}=\frac{p}{u}=\frac{p_{+}+p_{-} e^{i \beta}}{u_{+}-u_{-} e^{i \beta}}=\frac{p_{+}}{u_{+}} \frac{1+\frac{p_{-}}{p_{+}} e^{i \beta}}{1-\frac{u_{-}}{u_{+}} e^{i \beta}}=\rho_{0} c_{0} \frac{1+R_{-} e^{i \beta}}{1-R_{-} e^{i \beta}}
$$

where $R_{-}$is the ratio between the backward and the forward traveling wave defined as:

$$
R_{-}=\frac{p_{-}}{p_{+}}=\frac{u_{-}}{u_{+}}
$$

Equation (3.27) can be solved for the ratio $R_{-}$and the phase delay $\beta$ as they are pure real numbers:

$$
\begin{gathered}
R_{-}=\sqrt{\frac{\left(\rho_{0} c_{0}\right)^{2}-2 \rho_{0} c_{0} \operatorname{Re}\left(Z_{a c}\right)+\left|Z_{a c}\right|^{2}}{\left(\rho_{0} c_{0}\right)^{2}+2 \rho_{0} c_{0} \operatorname{Re}\left(Z_{a c}\right)+\left|Z_{a c}\right|^{2}}} \\
\beta=\operatorname{sign}\left(\operatorname{Im}\left(\mathrm{Z}_{\mathrm{ac}}\right)\right) \arccos \left(\frac{1}{R_{-}} \frac{\left|Z_{a c}\right|^{2}-\left(\rho_{0} c_{0}\right)^{2}}{\left(\rho_{0} c_{0}\right)^{2}+2 \rho_{0} c_{0} \operatorname{Re}\left(Z_{a c}\right)+\left|Z_{a c}\right|^{2}}\right)
\end{gathered}
$$

Knowing the frequency of the wave $f$, the ratio $R_{-}$and the phase delay $\beta$, the incoming wave can be calculated from historical data as follows:

$$
g(t, x)=R_{-} f\left(t-\frac{\beta}{2 \pi f}, x\right)
$$

In cases in which $\beta<0$, the boundary condition would need information from the future, thus the angle is corrected such that:

$$
\beta^{\prime}=\beta+2 \pi
$$


With this correction only data from the past is required to impose the incoming wave such that the acoustic impedance at the boundary corresponds to the acoustic impedance imposed $Z_{a c}$.

\subsubsection{Temperature boundary condition}

In addition to the pressure or the velocity, the temperature has to be imposed in CFD if the fluid enters the domain. As wave propagation also implies an oscillation in temperature, time dependent temperature changes need to be taken into account. As wave propagation is an adiabatic process, the temperature can be computed from the pressure assuming an adiabatic process:

$$
T\left(t, x_{b}\right)=T_{0} \cdot\left(1+\frac{p\left(t, x_{b}\right)}{p_{0}}\right)^{\frac{\gamma-1}{\gamma}}
$$

The acoustic boundaries have to be placed several displacements amplitudes away from all thermally interacting parts inside the domain such that the wave is adiabatic again at the boundaries. Whether this assumption is correct can be checked during the postprocessing by looking at the temperature over time at the boundary. If a jump in temperature occurs when the flow is reversed, the wave does not propagate adiabatically.

The temperature of the incoming fluid can also be set constant if an ideal heat exchanger shall be modeled with the boundary condition. In this case the incoming fluid has the temperature of the heat exchanger.

\subsubsection{Implementation}

The boundary condition is implemented via a User Defined Function in Fluent 14.0.0 [70] which calculates the pressure at the boundary and imposes it on the existing pressure boundary condition. The full $\mathrm{C}$ code can be split into different functions which are called at different moments during the calculation algorithm of ANSYS Fluent. Three categories can be distinguished: the initialization at the beginning, the functions to apply the boundary condition and finally the function for calculation of new values for the waves leaving the domain. They are presented in the following subchapters and summarized in Figure 3.6.

\subsubsection{Initialization function}

The initialization function is executed before the start of the calculation. The identification number of mesh points that are closest to the monitor point are saved, to avoid a scan of the entire domain at each future time step. Then the historical pressure data is set to zero and with this the pressure is calculated at the boundary for the first time step.

\subsubsection{Function to calculate the new pressure values}

In this function the pressure at the boundary for the following time step is calculated from the actual time data, once the actual time step is converged. The function is called at 
the end of each time step. As in most cases the monitor point does not lay on a cell center, the outgoing waves have to be interpolated from two cell centers. Therefore, an algorithm was written which finds the adjacent cell centers. At this location the outgoing traveling waves are calculated and afterwards interpolated according to the distance to the theoretical monitor point. With this data the pressure at the boundary is calculated according to Chapter 3.2.1 or 3.2.2.

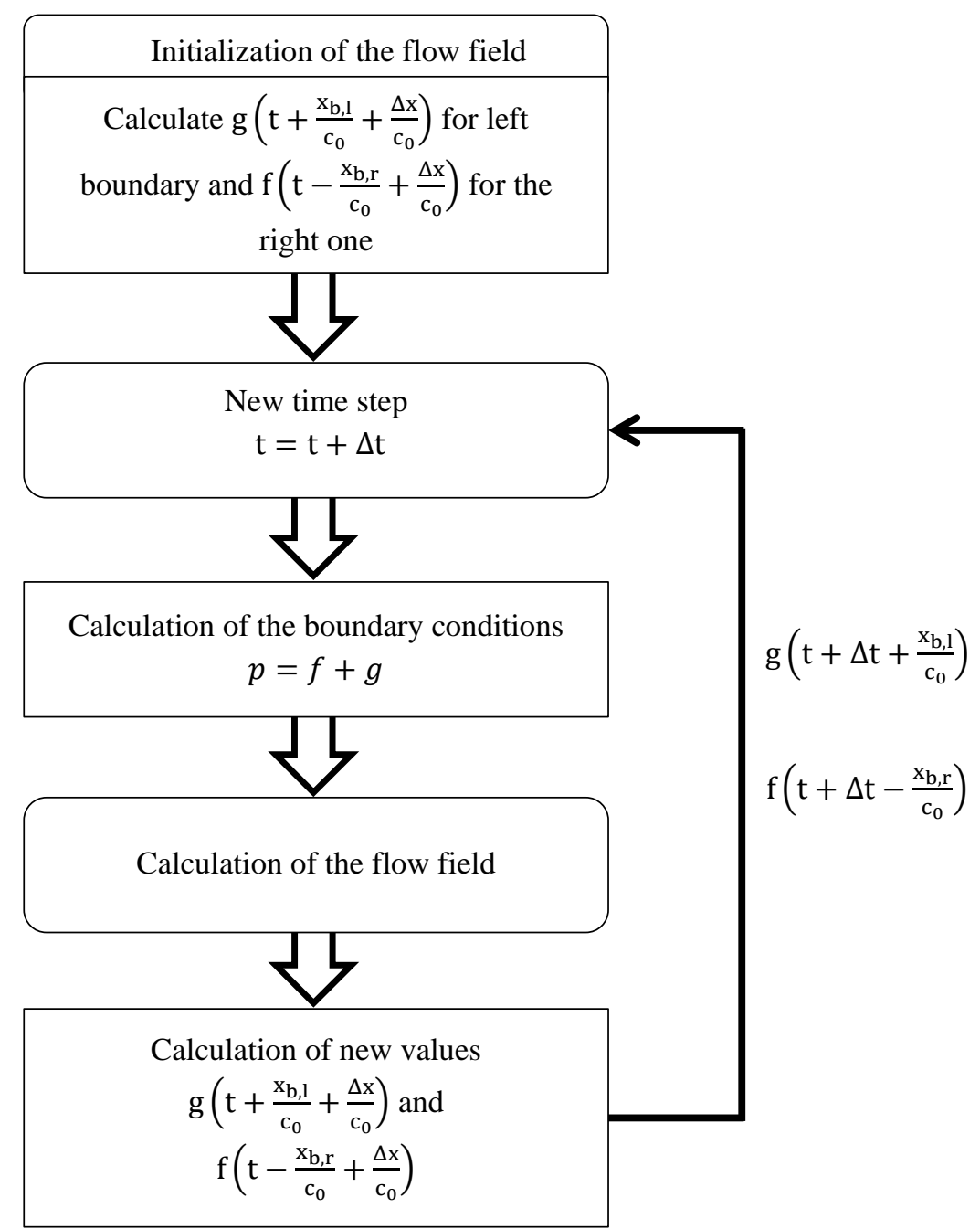

Figure 3.6: Calculation flow diagram of the non-reflecting boundary condition. Rectangles correspond to the implemented UDF's. 


\subsubsection{Function to apply the boundary condition}

The last type of function applies the pressure and temperature at the boundary. Here attention has to be paid to the fact that the pressure has to be applied in a different way, depending on whether the flow enters or leaves the domain. During the time when the flow enters the domain the total pressure and the total temperature have to be imposed:

$$
\begin{gathered}
p_{\text {total }}=\left(1+\frac{\gamma-1}{2} M^{2}\right)^{\frac{\gamma}{\gamma-1}}\left(p_{s}+p_{0}\right)-p_{0} \\
T_{\text {total }}=\left(1+\frac{\gamma-1}{2} M^{2}\right) T_{s}
\end{gathered}
$$

where $p_{s}$ is the static pressure and $T_{s}$ the static temperature from the implemented algorithm. However, when the flow leaves the domain, the static pressure can be applied directly.

\subsection{Limitation of thermoacoustic CFD simulations}

CFD simulations of thermoacoustic components suffer from a high computational cost. This has to do with the different scales that have to be resolved. Both in time and in space different orders of magnitude have to be resolved, as will be shown in the following.

\subsubsection{Spatial scales}

The biggest spatial dimensions in a thermoacoustic device are mostly on the order of the wavelength $\lambda$ :

$$
\lambda=\frac{c_{0}}{f}
$$

For a device running with helium at a frequency of $f=100 \mathrm{~Hz}$ and atmospheric pressure, the wavelength is roughly ten meters. The pipes in which the wave propagates, are already two orders of magnitude smaller and mostly on the order of centimeters. The smallest scale is defined by the viscous boundary layer $\delta_{v}$ :

$$
\delta_{v}=\sqrt{2 \frac{\mu}{\omega \rho}}
$$

as the thermoacoustic effect occurs close to the solid walls. For helium at $f=100 \mathrm{~Hz}$, $T=300 \mathrm{~K}$ and atmospheric pressure, the viscous penetration depth is:

$$
\delta_{v}=0.624 \mathrm{~mm}
$$

Several mesh points are needed inside the boundary layer, to resolve the momentum and heat transfer accurately. Hence, between the largest scale (the wavelength) and the smallest scale (the boundary layer elements close to the wall) there is a difference of approximately four orders of magnitude. This means that for the spatial discretization a very fine grid is needed on a large domain, which is computationally very expensive. 
Therefore, the spatial discretization has to be chosen carefully, as is discussed in Chapter 4 .

\subsubsection{Time scales}

Not only are the spatial dimensions spread over different orders of magnitude, but so are the time scales. In the investigated thermoacoustic systems, the frequency is $f=$ $100 \mathrm{~Hz}$, which corresponds to a period of $T=0.01 \mathrm{~s}$. Each period has to be resolved to several time steps. This is the smallest scale that has to be resolved, while the largest is the time that it takes to start a thermoacoustic device, which is on the order of minutes. Here again there are several orders of magnitudes between the smallest and largest scales, which makes the simulation computationally expensive. Ideas addressing this problem are presented in Chapter 4.

\subsubsection{Coupling between time and spatial discretization}

Due to the boundary condition presented in Chapter 3.2, the spatial and the time dimension are coupled. They are related by the CFL number [71]:

$$
C F L=c_{0} \cdot \frac{\Delta t}{\Delta x}
$$

which should be larger than one, but still close to it. Denoting $n_{t}$ and $n_{x}$ as the number of elements per period and per wavelength respectively, the time step size and the spatial element size can be written as:

$$
\begin{aligned}
\Delta t & =\frac{T}{n_{t}} \\
\Delta x & =\frac{\lambda}{n_{x}}
\end{aligned}
$$

Using that

$$
c_{0}=\lambda f=\frac{\lambda}{T}
$$

and assuming a CFL number of one, it follows:

$$
n_{t} \approx n_{x}
$$

This means that if a very small component is investigated, the time step size also has to be very small. This leads again to computationally expensive simulations. How to choose an appropriate discretization in order to reduce the computational costs is shown in the next chapter. 



\section{Validation for thermoacoustic simula- tions}

The derived low reduced frequency solutions from Chapter 2.3 are used in this chapter to conduct a verification and validation of the implemented boundary condition and numerical parameters in the sense of Laurien et al. [61]. In Chapter 4.1 the discretization parameters are investigated in the case of a vibrating resonator. This model has the advantage that it does not rely on sophisticated boundary conditions and allows an investigation of the discretization only. Afterwards, in Chapter 4.2 the non-reflecting boundary conditions and the appropriate discretization schemes are investigated. The impedance boundary conditions are studied in the one-dimensional case in chapter 4.3 and in the two-dimensional case in Chapter 4.4.

\subsection{Acoustic boundary layer discretization}

The analytical solution for a vibrating resonator derived in Chapter 2.3.3 is used here to validate numerical parameters in thermoacoustic simulations, especially the boundary layer discretization. A vibrating resonator is particularly suitable for this as no sophisticated boundaries are needed, only well-known no-slip boundary conditions. The analytical and numerical models are presented in Chapter 4.1.1, while the results for the best choice for spatial and time discretization are presented in Chapter 4.1.2.

\subsubsection{Shaking resonator models}

The shaking resonator test case is chosen because it allows the investigation of the interaction of an acoustic wave with a nearby wall, without using sophisticated boundary conditions in CFD. Furthermore, this model is easy to simulate as it is axisymmetric.

The resonator consists of a cylindrical tube filled with helium at atmospheric pressure (properties given in Appendix E). The resonator is harmonically shaken with a frequency of $f=100 \mathrm{~Hz}$ and a velocity amplitude of $u_{w}=10^{-4} \cdot c_{0}$. The velocity amplitude determines the energy introduced into the resonator and stored in the form of a standing wave. Energy is supplied until equilibrium between the introduced energy and the dissipation in the boundary layer is reached. For high velocity amplitudes of the resonator or large radii, reaching the steady periodic state can take a large number of periods. For this reason only small radii are simulated. The reference test case has a radius of $R=$ $0.005 \mathrm{~m}$, which corresponds to a shear wave number of $s=11.3$. The length of the tube is chosen to be half the wavelength $L=5.095 \mathrm{~m}$, in order to fulfill the assumptions of the low reduced frequency model. The dimensions and coordinates are summarized in Figure 4.1. 


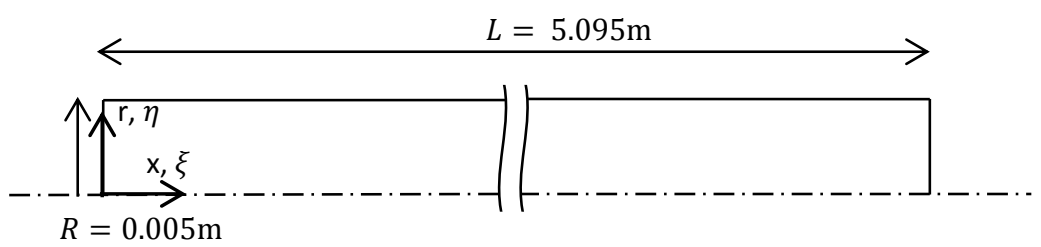

Figure 4.1: Dimensions and coordinates of the vibrating resonator.

The experiment can be characterized by the following dimensionless parameters:

- $\quad$ Shear wave number: $s=11.3$

- $\quad$ Square root of the Prandtl number: $\sqrt{P r}=0.824$

- $\quad$ Reduced frequency: $k=3.01 \cdot 10^{-3}$

- Specific heat ratio: $\gamma=1.6667$

- Excitation velocity: $\tilde{u}_{w}=10^{-4}$

Furthermore, it is assumed that helium behaves like an ideal gas with a constant dynamic viscosity and thermal conduction in both the CFD model and the low reduced frequency model.

\subsubsection{Low reduced frequency approximation}

The low reduced frequency approximation for a vibrating resonator presented in Chapter 2.3.3 is evaluated for the parameters given above. From this, the flow field in the resonator can be obtained. The model shows the steady, periodic state after an infinite amount of periods and allows the validation of the numerical parameters of the numerical models shown in the following chapter.

\subsubsection{Numerical model}

The numerical CFD model is implemented in ANSYS Fluent 13.0 [39]. The domain is modeled as two-dimensional axisymmetric, as no effects in the angular direction are expected. In order to reduce the calculation costs and to avoid a moving mesh, a change in coordinate system is done. Instead of carrying out the simulation in the global system, a local coordinate system on the resonator is defined. In the new coordinate system the movement of the tube can be seen as a volume force (see appendix F), which is applied in Fluent by a User Defined Function. In the local coordinate system all walls are assumed to be isothermal and with no-slip.

The reference test case is discretized with the following numerical parameters:

- Time step size $\Delta t=10^{-5}$ s. This corresponds to 1000 time steps per period.

- Elements in the axial direction $n_{x}=400$. This corresponds to 800 elements per wavelength.

- $\quad$ Elements in the radial direction $\mathrm{n}_{r}=10$. 
The reference parameters of the solver are given in Table 4.1. Furthermore, the convergence criteria are set to a residual of $10^{-9}$ for the energy equation and $10^{-5}$ for the continuity and momentum equations. To get the steady periodic solution first $0.2 \mathrm{~s}$ are simulated, which corresponds to 20 periods. The transient behavior of the axial velocity at the center of the resonator is shown in Figure 4.2. After the initial 20 periods, the data of the following 2 periods is exported and post processed for comparison with the low reduced frequency approximation.

Table 4.1: Solver Parameters.

\begin{tabular}{lll}
\hline Spatial discretization & Pressure & PRESTO! \\
& Density & Second Order Upwind \\
& Momentum & Second Order Upwind \\
& Energy & Second Order Upwind \\
\hline Transient Formulation & Time & Second Order Implicit \\
\hline Pressure Velocity Coupling & Coupling & PISO \\
\hline
\end{tabular}

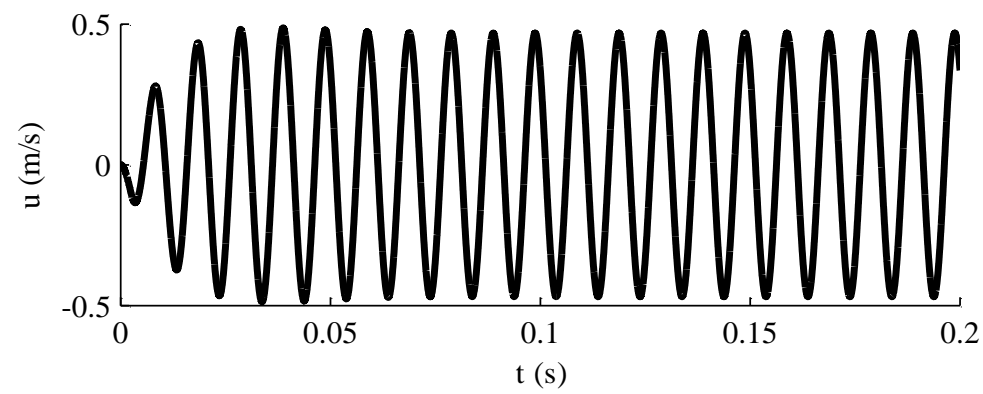

Figure 4.2: Transient oscillation of the axial velocity at the center of the resonator over the first 20 periods.

\subsubsection{Results}

The low reduced frequency model and the CFD model are compared in this section for different numerical parameters. This is done in order to find the best parameter set for thermoacoustic CFD simulations. The effect of the discretization of the boundary layer is investigated in Chapter 4.1.2.1 by looking at the discretization in radial direction. Afterwards, the time discretization is investigated in Chapter 4.1.2.2 by changing the time step size. Finally, the axial discretization is investigated in Chapter 4.1.2.3 by changing the 
number of elements per wavelength. Two major errors are expected if the numerical parameters are not appropriate. Numerical dissipation, which leads to a change in the amplitude of the flow variables. Dispersion, which leads to a change in phasing of the flow variables. Both are graphically defined in Figure 4.3.

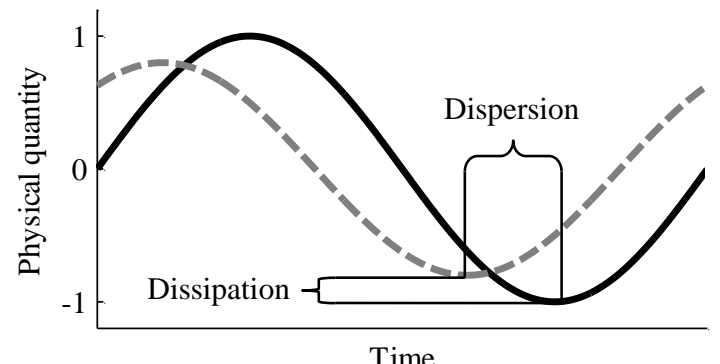

Figure 4.3: Definition of the dissipation and dispersion. Black solid line represents analytical solution and gray dashed line the numerical result.

\subsubsection{Radial discretization}

The first numerical parameter investigated is the influence of the mesh in the radial direction. The idea is to find a mesh size which allows an accurate solution to be calculated using as little numerical power as possible. The discretization of the vibrating resonator reference test case is therefore changed in the radial direction. First, different uniform meshes are investigated, with 10,20 and 50 elements over the height. The results for the axial velocity at the point $B$ at the center of the resonator (s. Appendix G) are shown in Figure 4.4. It can be seen that a phase delay and a difference in amplitude occurs for the coarsest meshes.

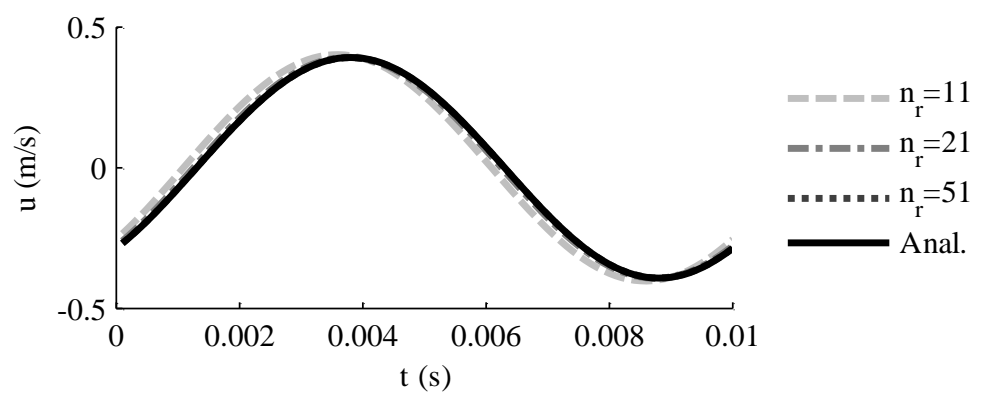

Figure 4.4: Axial velocity over time at location $B$ of the vibrating resonator for three uniform meshes, with 11, 21 and 51 nodes over the radius

In Table 4.2 the difference between the analytical solution and the CFD calculations of the different discretizations are given quantitatively. The refinement from 10 elements over the radius to 20 shows an improvement, as the phase delay and the amplitude differ- 
ence are reduced. A further increase in elements over the radius does not show a considerably better result.

Table 4.2: $\quad$ Phase delay and amplitude difference for three uniform meshes, with 11, 21 and 51 nodes over the radius.

\begin{tabular}{ccc}
\hline $\begin{array}{c}\text { Nodes over } \\
\text { the radius }\end{array}$ & Phase delay & $\begin{array}{c}\text { Relative difference in axial } \\
\text { velocity amplitude }\end{array}$ \\
\hline 11 & $-7.7^{\circ}$ & $2.6 \%$ \\
21 & $-1.8^{\circ}$ & $0.089 \%$ \\
51 & $-0.08^{\circ}$ & $-0.17 \%$ \\
\hline
\end{tabular}

In Figure 4.5 the axial velocity profile is plotted over the radius at the center of the resonator (line BB see Appendix G). The blue line represents the low reduced frequency solution and the dots show the corresponding values at the nodes of the different meshes. The profile is closely followed by the numerical results. It can be seen that for small radial locations the profile is nearly constant, while high gradients arise near the walls. Only a part of this gradient is inside the viscous boundary layer as defined in equation (3.3). In order to cover the complete domain where high gradients occur, an extended boundary layer is defined:

$$
\delta_{v, e x}=\delta_{v} \cdot \pi
$$

and plotted as the gray area in Figure 4.5. The factor $\pi$ is chosen, as the viscous penetration depth is defined as the characteristic length over which the momentum can diffuse during a time interval on the order of the period of the oscillation divided by $\pi$ [6].

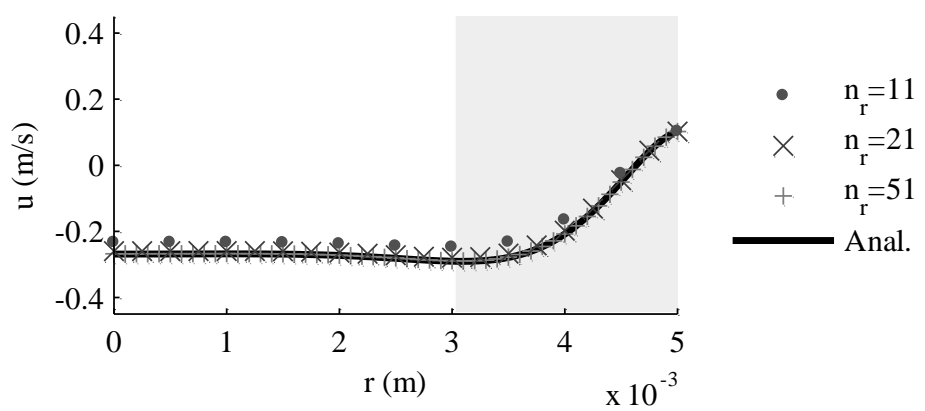

Figure 4.5: Axial velocity profile $u$ over the line BB in the middle of the resonator at the moment of maximum velocity of the resonator. Grayed area indicates the extended boundary layer $\delta_{v} \cdot \pi$.

The fact that the majority of changes occur in the part close to the wall and less occur near the axis indicates that a non-uniform grid is more appropriate: the nodes can be 
redistributed such that there are more elements close to the wall, where higher gradients occur compared to the center. A boundary layer discretization is therefore developed with the following parameters:

- Ten elements in the extended viscous boundary layer $\delta_{v, e x}$

- Ratio between two neighboring cell sizes is 1.2

Using this approach, only 17 nodes are needed over the radius. Due to the small elements at the wall a better convergence is achieved. Together with the reduced number of nodes, the simulation time can be reduced by $40 \%$ compared to the discretization with 21 nodes. The phase delay can be reduced to nearly zero and the amplitude error is smaller than $0.1 \%$. These results were also verified for different radii corresponding to shear wave numbers varying from $s=3$ to $s=45$ and the results are comparable.

The simulations presented in this chapter have shown that a non-appropriated discretization in the radial direction leads to considerable phase and amplitude deviations in the simulations. Due to the good results and the reduced simulation times, the boundary layer discretization is applied to the reference case in the following chapters instead of a uniform grid.

\subsubsection{Time discretization}

In this chapter the time discretization is investigated. Hence, the time step size is varied from $n_{T}=10$ elements per period $\left(\Delta t=1 \cdot 10^{-3} \mathrm{~s}\right)$ to $n_{T}=800$ elements per periods $\left(\Delta t=1.25 \cdot 10^{-5} \mathrm{~s}\right)$. The results in terms of phase delay and different amplitudes are summarized in Table 4.3. It can be seen that when the number of time steps per period is small, a large deviation in the amplitude of the axial velocity occurs. The table shows that from 100 time steps per period on, the relative error in velocity is low and a further reduction of the time step size does not results in a further improvement in the accuracy of the velocity phase and amplitude. However, it is expected that for wave propagation with less physical dissipation, a smaller time step will be needed as the relative numerical dissipation would increase. Furthermore, when the non-reflecting boundary condition is used, a smaller time step might be needed, especially when small components are investigated (see Chapter 3.2.1).

\subsubsection{Axial discretization}

The axial discretization is investigated in this chapter. Therefore, the number of elements per wavelength is varied from $n_{\lambda}=10$ to 400 . As the resonator is $\lambda / 2$ long, this corresponds to 5 to 200 elements in the axial direction of the numerical model. Table 4.4 shows the phase delay and the relative damping of the different axial discretizations. The results show that a discretization of 100 elements per wavelength is sufficient to obtain good results. These results are also checked for the temperature at one quarter of the resonator and a very similar trend is observed. The results of Table 4.4 show that 100 
elements per wavelength are enough for good results in the highly damped case with low shear wave number. This is not necessarily true in the case when less damping occurs, then more elements could be needed.

Table 4.3: $\quad$ Phase delay and amplitude difference for different time discretization.

\begin{tabular}{ccc}
\hline $\begin{array}{c}\text { Time steps } \\
\text { per period }\end{array}$ & Phase delay & $\begin{array}{c}\text { Relative difference of the } \\
\text { axial velocity amplitude }\end{array}$ \\
\hline 10 & $3.5^{\circ}$ & $-51.2 \%$ \\
20 & $4.6^{\circ}$ & $-18.6 \%$ \\
100 & $0.29^{\circ}$ & $-0.37 \%$ \\
200 & $0.064^{\circ}$ & $0.22 \%$ \\
400 & $0.015^{\circ}$ & $0.30 \%$ \\
800 & $0.015^{\circ}$ & $0.28 \%$ \\
\hline
\end{tabular}

Table 4.4: Phase delay and amplitude difference for different axial discretization.

\begin{tabular}{ccc}
\hline $\begin{array}{c}\text { Nodes per } \\
\text { wavelength }\end{array}$ & Phase delay & $\begin{array}{c}\text { Relative difference of the } \\
\text { axial velocity amplitude }\end{array}$ \\
\hline 10 & $2.1^{\circ}$ & $-27.2 \%$ \\
20 & $0.73^{\circ}$ & $-4.8 \%$ \\
50 & $0.083^{\circ}$ & $-0.67 \%$ \\
100 & $0.025^{\circ}$ & $0.19 \%$ \\
200 & $0.0048^{\circ}$ & $0.33 \%$ \\
400 & $-0.0013^{\circ}$ & $0.37 \%$ \\
\hline
\end{tabular}

\subsection{One-dimensional non-reflecting boundary condition - wave propagation}

The aim of the non-reflecting boundary condition presented in Chapter 3.2.1 is to model wave propagation in components of a thermoacoustic engine. While the time and spatial discretization of the acoustic boundary layer were investigated in the previous chapter, this chapter focuses on the verification and validation of the implemented nonreflecting boundary conditions. Thereby, the best discretization schemes for the nonreflecting boundary conditions are determined. 
The one-dimensional traveling wave model used to investigate the different implemented versions of the non-reflecting boundary conditions is presented in Chapter 4.2.1. The results are presented in Chapter 4.2.2.

\subsubsection{One-dimensional traveling wave model}

This model focuses on a one-dimensional plane traveling wave, which is propagating from one side of the domain to the other. This is done in order to investigate the performance of the non-reflecting boundary condition. The boundary conditions of the model are summarized in Figure 4.6. The wave enters the domain on one side by imposing the pressure at a non-reflecting boundary condition and exits at the other, through another non-reflecting boundary condition. The domain is $0.1 \mathrm{~m}$ high and $5 \mathrm{~m}$ long, which corresponds to roughly half a wavelength. The boundaries in the wave propagation directions are modeled as adiabatic and slip wall, to model one-dimensional adiabatic wave propagation.

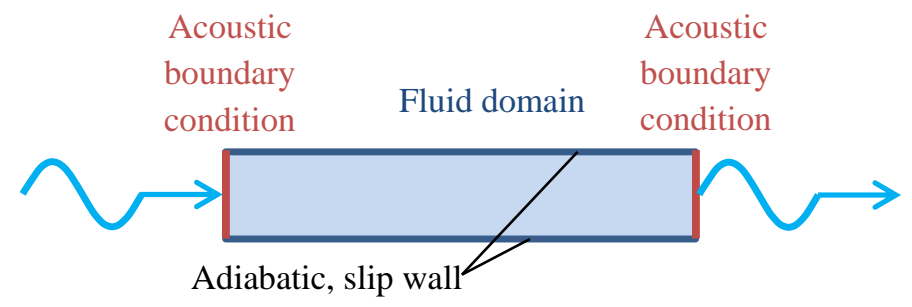

Figure 4.6: Boundary conditions for the one-dimensional wave propagation model.

Helium is chosen as a working gas as it is frequently used in thermoacoustic engines because of its low Prandtl number [12] and is modeled as ideal gas according the properties given in Appendix E. The incoming wave has a frequency of $f=100 \mathrm{~Hz}$ and pressure amplitudes $p_{1}$ of 100 and $10000 \mathrm{~Pa}$. These two amplitudes are chosen to model linear wave propagation and nonlinear wave propagation, respectively. The domain is discretized with quadrilateral elements, 200 elements in the wave propagation direction and four elements orthogonal to the wave propagation direction. The time step size is chosen to be fixed to $\Delta t=2 \cdot 10^{-5} \mathrm{~s}$, leading to 500 elements per period. In total 20 periods are simulated. Table 4.5 summarizes the parameters of this test case. 
Table 4.5: Parameters of one-dimensional traveling wave propagation test case and their value.

\begin{tabular}{lc}
\hline Parameter & Variable and value \\
\hline Length of the domain & $L=5 \mathrm{~m} \approx \frac{\lambda}{2}$ \\
Elements in wave propagation direction & $n_{x}=200$ \\
Elements orthogonal to wave propagation & $n_{y}=4$ \\
Frequency & $f=100 \mathrm{~Hz}$ \\
Time step size & $\Delta t=2 \cdot 10^{-5} \mathrm{~s}$ \\
Total time & $t=0.2 \mathrm{~s}=20 \cdot T$ \\
Pressure amplitude & $p_{1}=100 ; 10000 \mathrm{~Pa}$ \\
\hline
\end{tabular}

\subsubsection{Results}

In this chapter the results for one-dimensional wave propagation direction are presented. The influence of the numerical parameters on the wave propagation is investigated. First, the effect of the interpolation scheme is shown in Chapter 4.2.2.1. Then, the spatial discretization schemes are treated together in Chapter 4.2.2.2. For all simulations the following parameters are chosen unless otherwise stated:

Table 4.6: Initial numerical parameters of the test cases presented in this chapter.

\begin{tabular}{lc}
\hline \multicolumn{1}{c}{ Option } & Scheme \\
\hline Pressure interpolation scheme & Body Force Weighted \\
Density interpolation scheme & Second order \\
Convective terms in momentum equation & Second order upwind \\
Convective terms in energy equation & Second order upwind \\
\hline
\end{tabular}

\subsubsection{Interpolation scheme}

As ANSYS Fluent performs the calculations with a staggered grid, the pressure and density have to be interpolated from the cell center to the cell face. The pressure interpolation scheme has a larger effect on the solution, for this reason it will be discussed in more details. Five options are available in Fluent [39] and are summarized in Table 4.7. To check the influence of this parameter, five simulations of the test case described in 
Chapter 4.2.1 are performed only varying the pressure discretization scheme. The results are compared by means of the reflection coefficient at the non-reflecting outlet boundary condition, the drifting of the pressure over time and the damping of the pressure amplitude over the length of the domain.

Table 4.7: Pressure interpolation schemes [39].

\begin{tabular}{ll}
\hline Scheme & Explanation \\
\hline Standard & $\begin{array}{l}\text { Interpolates the pressure values at the faces using momen- } \\
\text { tum equation coefficients } \\
\text { Uses the discrete continuity balance for a "staggered" con- } \\
\text { trol volume about the face to compute the "staggered" face } \\
\text { pressure } \\
\text { CRESTO! }\end{array}$ \\
Linear & $\begin{array}{l}\text { values in the adjacent cells } \\
\text { Reconstructs the face pressure in the manner used for sec- } \\
\text { ond-order accurate convection terms } \\
\text { Computes the face pressure by assuming that the normal } \\
\text { gradient of the difference between pressure and body forc- } \\
\text { es is constant }\end{array}$ \\
Body Force & \\
Weighted &
\end{tabular}

First, the one-dimensional wave propagation boundary condition (see Chapter 3.2.1.1) is investigated at a pressure amplitude of $p_{1}=100 \mathrm{~Pa}$. The results are summarized in Table 4.8 .

Looking at the absolute value of the reflection coefficient, it can be concluded that the non-reflecting boundary condition is performing well. The reflection stays below 3\%, independently of the chosen pressure interpolation schemes, but the interpolation schemes Standard and PRESTO! show a higher drifting of the pressure and also a higher damping than the other schemes. This leads to a time averaged flow, which could influence heat transfer in later simulations by introducing unwanted convection.

The same simulations are conducted for the general plane wave propagation boundary condition and are summarized in Table 4.9. Again, the reflection coefficient stays below $3 \%$, which is satisfactory, but the interpolation schemes Standard, PRESTO! and especially Second order show a considerable drifting of the pressure over time. As this unwanted convection could alter future results, the Standard, PRESTO! and Second order schemes have to be avoided. 
Table 4.8: $\quad$ Results of the one-dimensional wave propagation test case with a pressure amplitude of $p_{1}=100 \mathrm{~Pa}$, using the one-dimensional wave propagation boundary condition at both extremities. The reflection, pressure drift and damping are given for the different pressure interpolation schemes.

\begin{tabular}{lccc}
\hline & $\begin{array}{c}\text { Reflection } \\
(\mathbf{a b s})\end{array}$ & $\begin{array}{c}\text { Drifting } \\
(\mathbf{P a} / \mathbf{s})\end{array}$ & $\begin{array}{c}\text { Damping } \\
(\mathbf{P a} / \mathbf{m})\end{array}$ \\
\hline Standard & $2,5 \%$ & 5,14 & $-0,0077$ \\
PRESTO! & $2,5 \%$ & 5,12 & $-0,0079$ \\
Linear & $2,5 \%$ & $-0,0002$ & 0,0002 \\
Second order & $1,8 \%$ & 0,49 & $-0,0002$ \\
Body Force & $2,5 \%$ & $-0,01$ & 0,0003 \\
Weighted & & & \\
\hline
\end{tabular}

Table 4.9: Results of the one-dimensional wave propagation test case with a pressure amplitude of $p_{1}=100 \mathrm{~Pa}$, using the general plane wave propagation boundary condition at both extremities. The reflection, pressure drift and damping are given for the different pressure interpolation schemes.

\begin{tabular}{lccc}
\hline & $\begin{array}{c}\text { Reflection } \\
(\mathbf{a b s})\end{array}$ & $\begin{array}{c}\text { Drifting } \\
(\mathbf{P a} / \mathbf{s})\end{array}$ & $\begin{array}{c}\text { Damping } \\
(\mathbf{P a} / \mathbf{m})\end{array}$ \\
\hline Standard & $2,5 \%$ & 2,67 & 0,0004 \\
PRESTO! & $2,5 \%$ & 2,20 & 0,0002 \\
Linear & $2,5 \%$ & $-0,07$ & 0,0002 \\
Second order & $2,7 \%$ & $-47,41$ & 0,0453 \\
$\begin{array}{l}\text { Body Force } \\
\text { Weighted }\end{array}$ & $2,5 \%$ & $-0,03$ & 0,0002 \\
\hline
\end{tabular}

Comparing both boundary conditions shows that the boundary condition for general plane wave propagation is much more sensible for the choice of numerical parameters as it leads to a very large amount of drifting for some schemes. This can be explained by the fact that this boundary condition accumulates errors with time, which leads to drifting. Even though the results obtained using this boundary are not encouraging, a deeper look at this boundary is undertaken, as some cases like pipe flow, can only be simulated with the general plane wave boundary condition. As it demands the most attention, results in the following are only presented for this boundary condition.

If the pressure amplitude is increased to $p_{1}=10000 \mathrm{~Pa}$, the difference between the pressure interpolation schemes becomes larger. The results are summarized in Table 4.10. 
Choosing for the Second order scheme leads to a higher reflection at the boundary as well as large pressure drifting over time compared to the other schemes . The Body Force Weighted scheme performs best with a reflection coefficient of $2.1 \%$ and the lowest drifting. The same can be observed for the one-dimensional wave propagation boundary condition (not shown here). For this reason all further simulations will be performed with the Body Force Weighted scheme for pressure interpolation.

Table 4.10: Results of the one-dimensional wave propagation test case with a pressure amplitude of $p_{1}=10000 \mathrm{~Pa}$, using the general plane wave propagation boundary condition at both extremities. The reflection, pressure drift and damping are given for the different pressure interpolation schemes.

\begin{tabular}{lccc}
\hline & $\begin{array}{c}\text { Reflection } \\
(\mathbf{a b s})\end{array}$ & $\begin{array}{c}\text { Drifting } \\
(\mathbf{P a} / \mathbf{s})\end{array}$ & $\begin{array}{c}\text { Damping } \\
(\mathbf{P a} / \mathbf{m})\end{array}$ \\
\hline Standard & $2,0 \%$ & $-2296,84$ & 8,2 \\
PRESTO! & $1,8 \%$ & $-770,59$ & 15,3 \\
Linear & $2,1 \%$ & $-441,16$ & 3,1 \\
Second order & $6,1 \%$ & $-70783,67$ & 79,5 \\
Body Force & $2,1 \%$ & $-65,45$ & 2,7 \\
Weighted & & & \\
\hline
\end{tabular}

The influence of the density interpolation scheme on the results is less severe compared to the pressure interpolation scheme. The effect of the density interpolation scheme on the reflection coefficient and damping inside the domain is negligible. However, the interpolation scheme Second Order Upwind performs best with regard to the drifting. For this reason it is chosen for all following simulations.

To conclude, due to numerical drifting of the pressure in time occurring for some schemes, the interpolation scheme has to be selected carefully. It is shown that choosing the Body Force Weighted scheme for pressure interpolation and the Second Order Upwind scheme for the density interpolation leads to reasonable results.

\subsubsection{Spatial discretization schemes}

Next to the interpolation schemes presented in the previous chapter ANSYS Fluent allows the selection of different schemes for the first order derivatives and the discretization of the convection terms in the momentum and energy equation. Diffusion terms are set to second order accurate centered difference [62].

As shown in the previous chapter, the differences between the numerical parameters are biggest when the general plane wave boundary condition is selected with a wave of pressure amplitude of $p_{1}=10000 \mathrm{~Pa}$. Therefore, the discussion in the following is restricted to this case, which is most demanding. 
The results of the different simulations varying the spatial discretization scheme are summarized in Table 4.11. The choice does not have any influence on the reflection coefficient and the damping of the wave within the length of the domain stays below $3 \mathrm{~Pa} / \mathrm{m}$. On the other hand, the drifting is highly dependent on the choice of the scheme. The Green-Gauss Node-Based scheme leads to an unacceptable drifting, while the Least Squares Cell-based scheme gives the best results. Thus, the scheme for spatial discretization is not changed from the standard value of ANSYS Fluent, the Least Squares Cellbased scheme [62].

Table 4.11: Results of the one-dimensional wave propagation test case with a pressure amplitude of $p_{1}=10000 \mathrm{~Pa}$, using the general plane wave propagation boundary condition at both extremities. The reflection, pressure drift and damping are given for the different spatial discretization schemes.

\begin{tabular}{lccc}
\hline & $\begin{array}{c}\text { Reflection } \\
(\mathbf{a b s})\end{array}$ & $\begin{array}{c}\text { Drifting } \\
(\mathbf{P a} / \mathbf{s})\end{array}$ & $\begin{array}{c}\text { Mean damping } \\
(\mathbf{P a} / \mathbf{m})\end{array}$ \\
\hline Least Squares Cell-based & $2,1 \%$ & $-65,45$ & 2,7 \\
Green-Gauss Cell-Based & $2,1 \%$ & $-660,15$ & 3,4 \\
Green-Gauss Node-Based & $2,1 \%$ & 2128,40 & $-0,4$ \\
\hline
\end{tabular}

In the next step the discretization scheme of the convective terms in the momentum and the energy equation will be compared. As the available schemes are the same and the results are comparable, both results are discussed together. The First Order Upwind scheme leads to a high drift in pressure. This is possibly caused by the high amount of numerical diffusion introduced with this scheme [72], which cloud lead to errors in the evaluation of the boundary condition which add up. The Second Order Upwind scheme reduces numerical diffusion, which is believed to be the reason for the smaller numerical drifting observed using this scheme. This parameter is chosen for the further simulations, as the results with this scheme present the smallest amount of drifting.

In the previous two sections the best schemes for the simulation of plane wave propagation could be derived by looking at the reflection coefficient, the drifting of the pressure over time and the damping over the length of the domain. The best parameters are summarized in Table 4.6.

\subsubsection{Influence of the wave propagation}

The drifting of the pressure with time observed in the simulation was investigated in the previous section with respect to the numerical parameters. An incorrect wave propagation model used to extrapolate the pressure from the monitor point to the boundary could also lead to errors and thus to a pressure drift with time. For this reason five differ- 
ent wave propagation models are compared in this chapter. The underlying assumptions and the model equations are summarized in Table 4.12.

Table 4.12: Wave propagation models with their underlying idea and model equation.

\begin{tabular}{cc}
\hline \multicolumn{1}{c}{ Model idea } & Model equation \\
\hline $\begin{array}{c}\text { Wave propagates with the unperturbed speed of } \\
\text { sound }\end{array}$ & $c=c_{0}=\sqrt{\gamma R_{S} T_{0}}$ \\
Wave propagation speed depends on temperature & $c=c_{1}=\sqrt{\gamma R_{S} T}$ \\
Wave propagation speed dependents on convec- \\
tive effects (sign depending on whether the wave \\
is traveling forward or backward) \\
$\begin{array}{c}\text { Wave propagation speed depends on temperature } \\
\text { and convective effects }\end{array}$ \\
$\begin{array}{c}\text { Wave propagation model for nonlinear wave } \\
\text { propagation from [73], where } \beta \text { is the coefficient } \\
\text { of nonlinearity }\end{array}$ & $c=c_{0} \pm u$ \\
\hline
\end{tabular}

The results of this investigation are summarized in Table 4.13 for a wave with a high pressure amplitude of $p_{1}=10000 \mathrm{~Pa}$. At this amplitude nonlinear effects can occur [50]. It can be seen that the reflection coefficient is slightly reduced when the model accounts for convection and the temperature dependency. This shows, that incorporating the nonlinear effects leads to a more accurately predicting of the wave propagation between the monitor point and the boundary. However, while the reflection coefficient decreases only slightly, the drifting increases in a larger amount. This cannot be easily explained. Poinsot and Lele [74] also faced pressure drifting over time with their non-reflecting boundary condition. They explain it with the fact that the better the non-reflecting boundary condition works, the less information from the far field can enter the domain. This leads to a lack of information for the mean pressure and it drifts away. Indeed, looking at all simulations that were performed, those simulations with a smaller reflection coefficient show a higher pressure drift compared to simulations with higher reflection coefficient. This means that the drifting could have two causes, first the accumulation of numerical errors and second that no information about the far field can enter the domain to restore the mean value. Because of the results described above, the wave propagation 
speed will be set to the unperturbed speed of sound, which simplifies the non-reflecting boundary condition, because the monitor points stay constant location.

Table 4.13: Results of one-dimensional wave propagation with general plane wave propagation boundary condition using wave propagation models. Pressure amplitude $p_{1}=10000 \mathrm{~Pa}$.

\begin{tabular}{cccc}
\hline $\begin{array}{c}\text { Wave propagation } \\
\text { speed }\end{array}$ & $\begin{array}{c}\text { Reflection } \\
(\mathbf{a b s})\end{array}$ & $\begin{array}{c}\text { Drifting } \\
(\mathbf{P a} / \mathbf{s})\end{array}$ & $\begin{array}{c}\text { Mean damping } \\
(\mathbf{P a} / \mathbf{m})\end{array}$ \\
\hline $\mathrm{c}=\mathrm{c}_{0}$ & $2,1 \%$ & -65 & 2,7 \\
$\mathrm{c}=\mathrm{c}_{1}$ & $2,0 \%$ & -453 & 2,7 \\
$\mathrm{c}=\mathrm{c}_{0}+\mathrm{u}$ & $2,0 \%$ & -1381 & 2,6 \\
$\mathrm{c}=\mathrm{c}_{1}+\mathrm{u}$ & $1,9 \%$ & -1787 & 2,7 \\
$\mathrm{c}=\mathrm{c}_{0}+\beta \mathrm{u}$ & $1,9 \%$ & -1853 & 2,6 \\
\hline
\end{tabular}

Next to the wave propagation, the plane wave averaging (see Chapter 3.2.1.3.3) was also investigated, but no noticeable effect could be seen. Plane wave averaging can be used for meshes with a lot of elements in the direction orthogonal to the wave propagation direction to reduce the amount of memory used, as only the averaged value of the pressure at every time step is stored. Furthermore, averaging has the advantage that no numerical oscillations on the boundary itself can occur, which makes the simulations more stable, especially when non acoustic flow structures impinge on the boundary, such as vortices.

\subsection{One-dimensional impedance boundary condition}

In this chapter the impedance boundary conditions are investigated in the case of onedimensional wave propagation. Just as with the non-reflecting boundary condition, two boundaries can be differentiated. The first boundary condition, that assumes onedimensional wave propagation and the second more general one that assumes general plane wave propagation. Both can be applied to the one-dimensional model presented in Chapter 4.3.1. The results are given in Chapter 4.3.2.

\subsubsection{One-dimensional standing wave resonator model}

This test case is meant to validate the impedance boundary condition. A wave enters the domain, travels through it and gets reflected at the end wall at $L=5 \mathrm{~m}$. The reflected wave travels back to where it entered the domain and exits there without reflection. A standing wave builds up. A preliminary simulation with the whole domain is done as reference. From this reference case, the impedance at the center of the tube is evaluated. This allows a reduction of the computational domain to half of the resonator, by imposing 
an impedance boundary-condition at the right side of the reduced domain. Comparing both simulations allows for a quantification of the performance of the impedance boundary condition. In the ideal case, both simulations should overlay each other.

Figure 4.7 summarizes the boundary conditions for the reference case. The physical and numerical parameters are chosen to be the same as in the previous chapter (see Table 4.5) for the whole resonator. In the case of the half resonator only 100 elements are taken in the axial direction to have a comparable element size to the reference case.

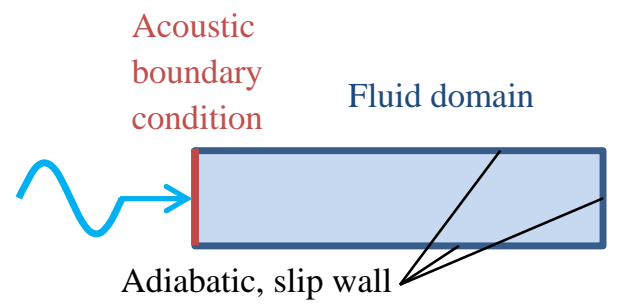

Figure 4.7: Boundary conditions for the one-dimensional wave propagation test case.

\subsubsection{Results}

In this chapter the impedance boundary conditions are tested. The model consists of a one-dimensional resonator as was described in Chapter 4.3.1. For both impedance boundary conditions two simulations are performed. The first simulation models the entire resonator with the reflecting wall (no-slip) at the end and is referenced as "Reference case". It does not use an impedance boundary condition. The second simulation models only the first half of the resonator, the second half is modeled by the impedance boundary condition. Both results are compared with the analytical solution. Comparing both results with the analytical solution allows the isolation of the additional errors introduced by the impedance boundary condition only. The pressure and the velocity are monitored at a point one meter from the inlet in all simulations. The deviation of the simulation results from the analytical solution are compared and shown in Figure 4.8 and Figure 4.9.

It can be seen that there is a good agreement of the simulation results with the analytical solution. In all cases the deviation is smaller than 3\%. The deviations from the analytical model do not increase compared to the "Reference case" when using an impedance boundary condition. It can be concluded that the impedance boundary condition does not add additional errors. Comparing both impedance boundary conditions shows that the deviation for the general plane wave non-reflecting boundary conditions are larger as drifting occurs. This is true for both domains, the full one and the reduced one. For this reason the one-dimensional plane wave boundary condition should be used were possible. Nevertheless, the general plane wave impedance boundary condition performs well, as errors stay smaller than $3 \%$. It can thus be concluded that both impedance boundary conditions are correctly implemented and work well. 


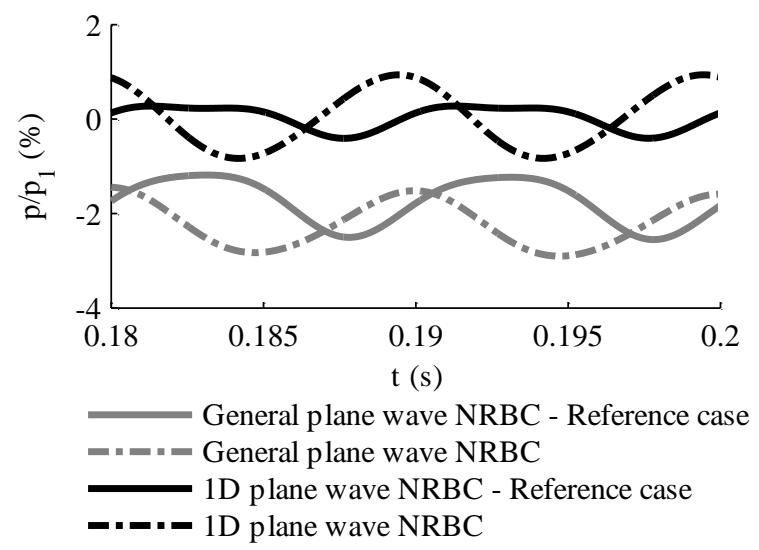

Figure 4.8: Normalized deviation of the velocity from the analytical solution over time at a monitor point one meter from the inlet of the resonator for the two impedance boundary conditions and their reference cases.

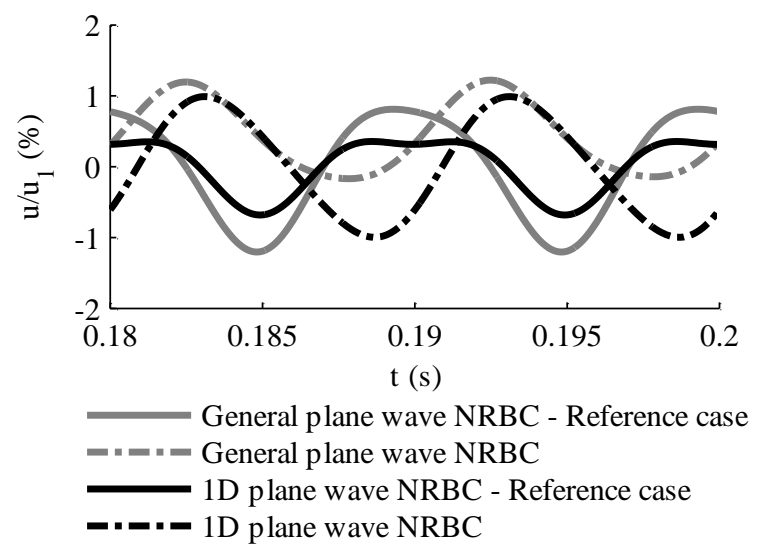

Figure 4.9: Normalized deviation of the pressure from the analytical solution over time at monitor point one meter from the inlet of the resonator for the two impedance boundary conditions and their reference cases.

\subsection{Two-dimensional impedance boundary condition}

The general plane wave impedance boundary condition is investigated in this chapter in the case of two-dimensional wave propagation. In this case the velocity has a twodimensional character, for example due to a nearby boundary layer. But the pressure is constant orthogonal to the wave propagation direction. The model is presented in Chapter 4.4.1 and the results summarized in Chapter 4.4.2. 


\subsubsection{Two-dimensional wave propagation model}

In this chapter the general plane wave impedance boundary condition, as defined in Chapter 3.2.1.2, is applied to two-dimensional wave propagation in a small cylindrical channel. The working gas is helium (Appendix E). The channel has a length of $L=5 \mathrm{~m}$ which is divided into 500 elements and a radius of $R=0.01 \mathrm{~m}$, discretized with 19 elements growing from the wall at a growth rate of $q=1.2$. The shear wave number characterizing the model is $s=22.6$. The wall is assumed to be isothermal and no-slip. In the first test case the purely non-reflecting boundary condition is tested. The wave enters the domain at the left and leaves it on the right side. In a second test case, the impedance boundary condition is tested by introducing a wave on both sides with different amplitude and phasing. The boundary conditions are summarized in Figure 4.10 and the parameters for both simulations in Table 4.14.

Table 4.14: Parameters of two-dimensional test cases and their values.

\begin{tabular}{lc}
\hline Parameter & Variable and value \\
\hline Length of the domain & $L=5 \mathrm{~m} \approx \frac{\lambda}{2}$ \\
Elements in wave propagation direction & $n_{x}=500$ \\
Elements orthogonal to wave propagation & $n_{y}=19$ \\
Growth rate in radial direction & $q=1.2$ \\
Frequency & $f=100 \mathrm{~Hz}$ \\
Time step size & $\Delta t=2 \cdot 10^{-5} \mathrm{~s}$ \\
Total time & $t=0.2 \mathrm{~s}=20 \cdot T$ \\
Pressure amplitude & $p_{1}=100 \mathrm{~Pa}$ \\
\hline
\end{tabular}




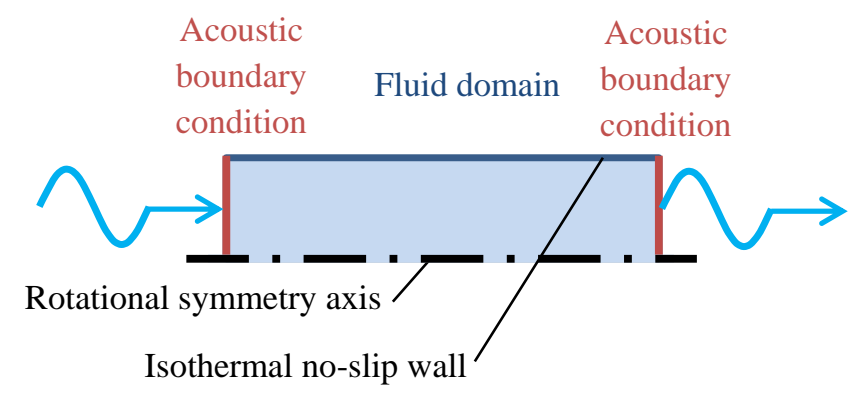

Figure 4.10: Boundary conditions for the two-dimensional wave propagation test case.

\subsubsection{Results}

In this chapter two-dimensional wave propagation is investigated, thus only the general plane wave, non-reflecting boundary condition can be applied. In the first simulation the wave is entering at the left side and exiting at the right through a non-reflecting boundary condition. The results are compared with the low reduced frequency model of a cylindrical tube presented in Chapter 2.3.2.

First the time dependent behavior is investigated. The pressure and the axial velocity are monitored at the center of the tube $(R=0)$ and at one half of the total length $(x=$ $L / 2$ ). The deviation of the simulation results from the low reduced frequency model are normalized with the amplitude and shown in Figure 4.11 and Figure 4.12. While the pressure deviation is smaller than $3 \%$, the velocity deviation starts with a higher deviation of up to $10 \%$, but with time it converges to be within $2 \%$.

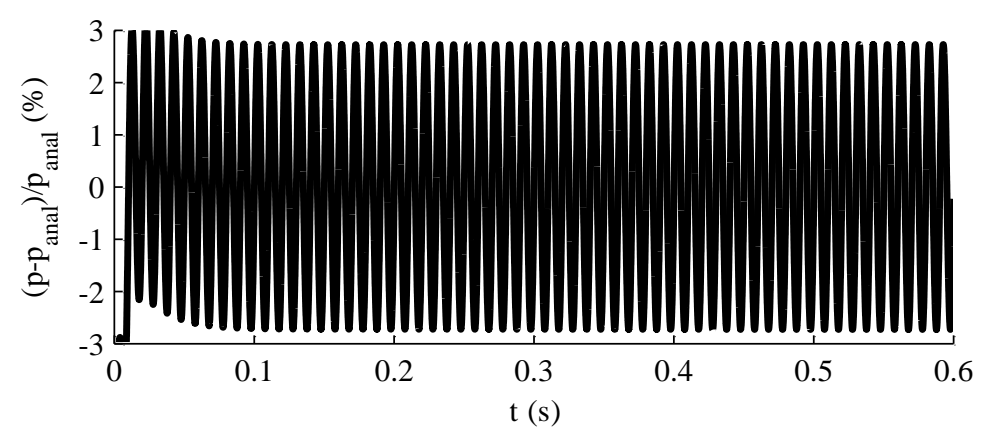

Figure 4.11: Normalized pressure deviation of the simulation from the low reduced frequency model at a monitor point at the center of tube $(x=L / 2 ; R=0)$ over time. 


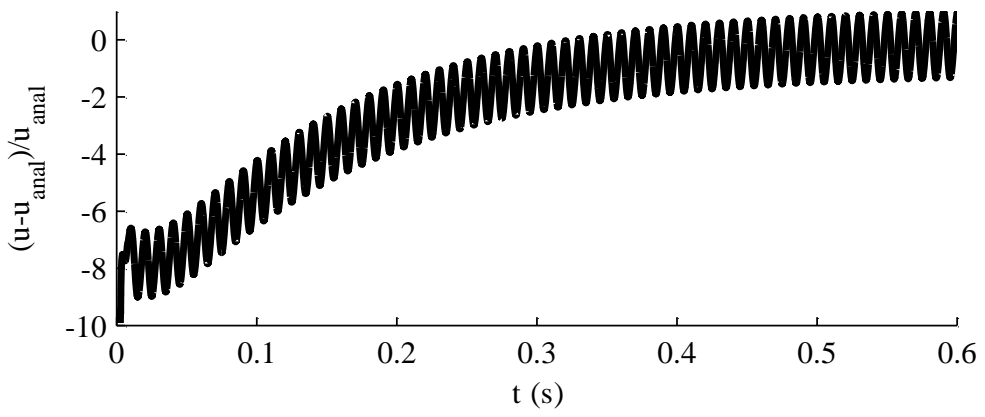

Figure 4.12: Normalized velocity deviation of the simulation from the low reduced frequency model at a monitor point at the center of tube $(x=L / 2 ; R=0)$ over time.

In the following, the radial distribution of the different flow variables is shown at four time instances. The pressure, density and temperature are nearly overlaying the solution of the low reduced frequency model. To illustrate the level of agreement, Figure 4.13 shows the density over the radius at four instances. While the pressure, density and temperature show a very close agreement with the low reduced frequency model, the axial (Figure 4.14) and radial velocity (Figure 4.15) deviate slightly. The results of the axial velocity are in agreement with the results shown in Figure 4.12. The agreement becomes better with increasing simulation time. While the profile of the axial velocity is qualitatively correct, the radial profile shows a non-zero velocity value for $R=0$, which should not be the case due to the symmetry axis at this location. This could be numerical inaccuracy due to the large aspect ratio of the cells and the fact that the radial velocity is more than three orders of magnitude smaller than the axial velocity. However, future investigations are focusing mainly on momentum and heat transfer at the wall. For these investigations, the profiles at the wall are determining. As the results are closely matching at the wall, we can conclude that the method is appropriate.

In a second test case a second wave is additionally imposed at the right side of the domain. This models an impedance boundary condition. The results show the same behavior as compared to the case where a wave is only entering at the left.

To conclude, in this chapter it is shown that CFD is capable of modeling acoustic phenomena by comparing the simulations with analytical solutions. It is shown which parameters lead to good results concerning the discretization of a boundary layer and the propagation of a wave. Furthermore, it is shown that the implemented boundary conditions are able to reduce the computational domain such that components of thermoacoustic devices can be investigated in more detail. The aforementioned validation is used in the following chapter to investigate components of different size within a thermoacoustic device. 


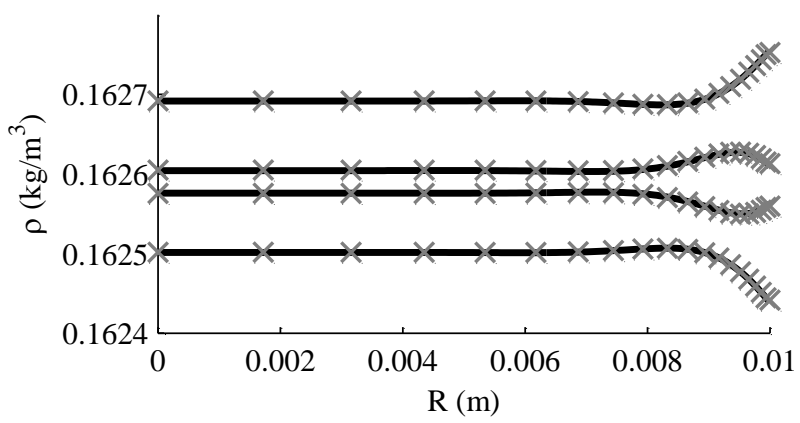

Figure 4.13: Density over the radius at four instances of a period, separated by a quarter period each. Black lines gray crosses represent the analytical solution and the numerical solution respectively.

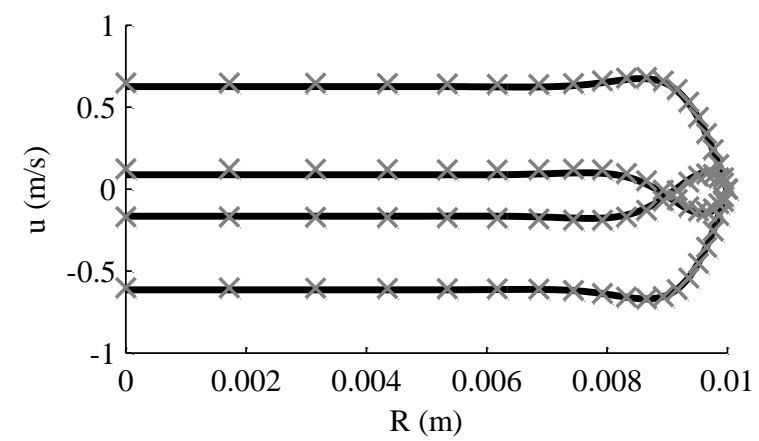

Figure 4.14: Axial velocity over the radius at four instances of a period, separated by a quarter period each. Black lines gray crosses represent the analytical solution and the numerical solution respectively.

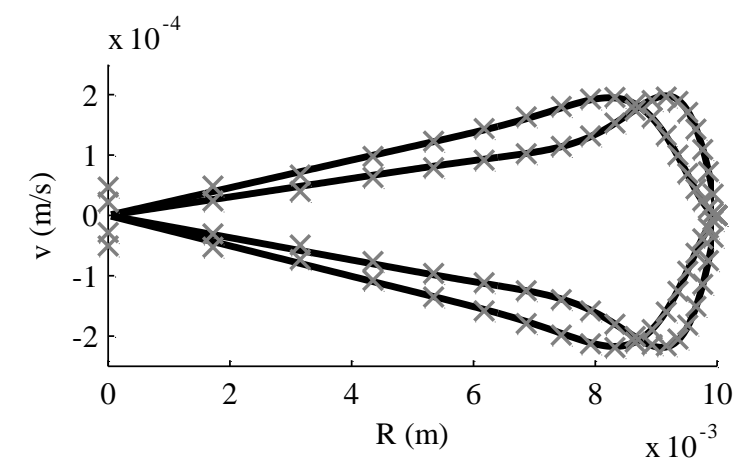

Figure 4.15: Radial velocity over the radius at four instances of a period, separated by a quarter period each. Black lines gray crosses represent the analytical solution and the numerical solution respectively. 


\subsection{Ideal heat exchanger boundary condition}

The ideal heat exchanger boundary condition is investigated in this chapter. An ideal heat exchanger is defined by an infinitely large heat transfer coefficient and no viscous effects inside the heat exchanger. For this reason the ideal heat exchanger boundary condition only differs compared to the previously presented boundary condition, in the temperature imposed while the flow is entering the domain. The temperature is not calculated from the pressure assuming an adiabatic process, but is set constant to the temperature of the modeled heat exchanger. With this boundary condition it is possible to investigate, for example, a thermal buffer tube on its own without fully modeling the heat exchangers (see Chapter 5.1).

The validation of the boundary condition is done by comparing the mean temperature in the vicinity of the heat exchanger with an analytical solution developed for predicting the mean temperature in a thermal buffer tube. The analytical solution and the CFD model are presented in Chapter 4.5.1. The results are given in Chapter 4.5.2.

\subsubsection{Ideal heat exchanger models}

In order to validate the ideal heat exchanger boundary condition two models are compared. First in Chapter 4.5.1.1 an analytical solution for the mean temperature close to an ideal heat exchanger is given, while the CFD model is presented in the subsequent chapter.

\subsubsection{Analytical solution}

An analytical solution is used to validate the ideal heat exchanger boundary condition. The mean temperature is selected as a benchmark quantity as it is the result of a combined interaction of the acoustic flow field with the ideal heat exchanger. This change in mean temperature profile was experimentally reported by Storch et al. [75]. The mean temperature profile does not follow the linear thermoacoustic theory derived by Rott [1] and reviewed by Swift [6]. This is due to the violation of the assumption that the displacement amplitude $\xi_{1}$ is much smaller than all other relevant dimensions in the wave propagation direction. However, in common thermoacoustic refrigerators the length of the heat exchanger is comparable with the displacement amplitude $\xi_{1}$ [6]. In this case the convective effects $(u \cdot \nabla) T$ at the entrance of the heat exchangers are not negligible as they lead to a change in mean temperature, which can be on the order of the adiabatic temperature oscillation. This change in mean temperature is used to validate the boundary condition. A simple explanation of this phenomenon can be found in Swift [6].

Analytical solutions were derived by Matveev et al. [76] and Gusev et al. [77] for simplified cases. The model used here is the model derived by Matveev et al. [76]. The main assumptions for the derivation are:

- No viscous wall effects occur, one-dimensional acoustics. 
- No heat conduction besides the temperature gradient imposed by the heat exchangers.

- The pressure is spatially constant.

For the validation of the ideal heat exchanger boundary condition a pure traveling wave is investigated. Furthermore, only one heat exchanger is investigated, so there is no temperature gradient. In this case the analytical solution for the mean temperature can be written as [78]:

$$
\Delta T_{0}(x)=-\frac{2}{\pi}\left(1-\left(\frac{x}{2 \xi_{1}}\right)^{\frac{\pi}{2}}\right) \frac{\gamma-1}{\gamma} \frac{p_{1}}{p_{0}} T_{0}
$$

\subsubsection{CFD model}

The test case to validate the ideal heat exchanger boundary condition consists of an ideal heat exchanger with an adjacent open area. The model and the boundary conditions are presented in Figure 4.16. The heat exchanger at the left of the domain is modeled with the ideal heat exchanger boundary condition. The boundary condition imposes the pressure such that a traveling wave is introduced at the left of the domain with a pressure amplitude of $p_{1}=100 \mathrm{~Pa}$. The temperature of the incoming fluid is set to the heat exchanger temperature $T=300 \mathrm{~K}$. The wave introduced at the left of the domain travels through the computational domain and exits at the right, through a non-reflecting boundary condition (see Chapter 4.2). The horizontal boundary conditions are set to be periodic. The total domain is five displacement amplitudes $\xi_{1}$ long and 0.04 displacement amplitudes $\xi_{1}$ high. The domain is discretized by 100 elements per displacement amplitude in both spatial directions.

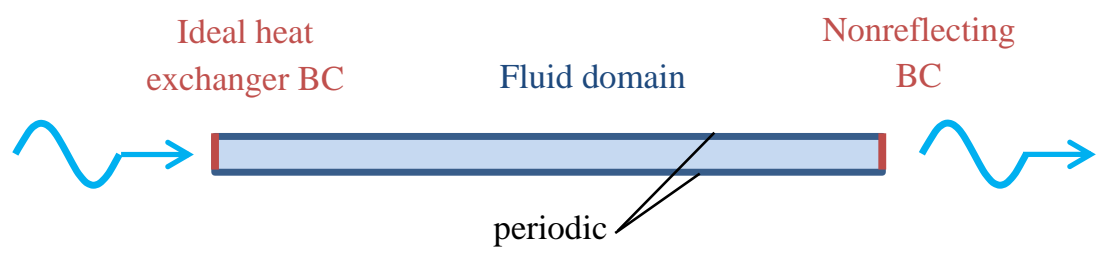

Figure 4.16: Boundary conditions of the ideal heat exchanger validation model.

The working fluid is helium at a mean pressure of $p_{0}=1 \mathrm{~atm}$ and at a temperature of $T_{0}=300 \mathrm{~K}$ (see Chapter E). The introduced wave has a frequency of $f=100 \mathrm{~Hz}$. In total five periods are simulated and the mean temperature is calculated by averaging the last period.

\subsubsection{Results}

The mean temperature profiles versus the dimensionless $x$-coordinate for two simulations are plotted in Figure 4.17. The green line shows the mean temperature profile with zero heat conductivity. In this case the effect of the ideal heat exchanger only extends two 
displacement amplitudes $\xi_{1}$ away from the heat exchanger. The blue dotted line shows the mean temperature assuming a heat conductivity of $\mathrm{k}=0.152 \mathrm{~W} / \mathrm{mK}$ corresponding to helium (see Chapter E). When heat conduction is taken into account in the simulation, the minimum temperature is smoothed out. A clear minimum in the mean temperature can be seen within one displacement amplitude of the ideal heat exchanger. Heat is conducted into the rest of the domain and the mean temperature profile is influenced beyond two displacements amplitudes $\xi_{1}$ away from the heat exchanger. The figure also indicates that additional thermal losses are introduced due to the conduction over the right boundary, which was also described by Storch et al. [75].

The black dashed line in Figure 4.17 shows the analytical solution given in Equation (4.2). The analytical solution overlays the green line as in both cases no heat conduction is assumed. It can be concluded that the applied boundary condition is correctly implemented and that the boundary can model an ideal heat exchanger.

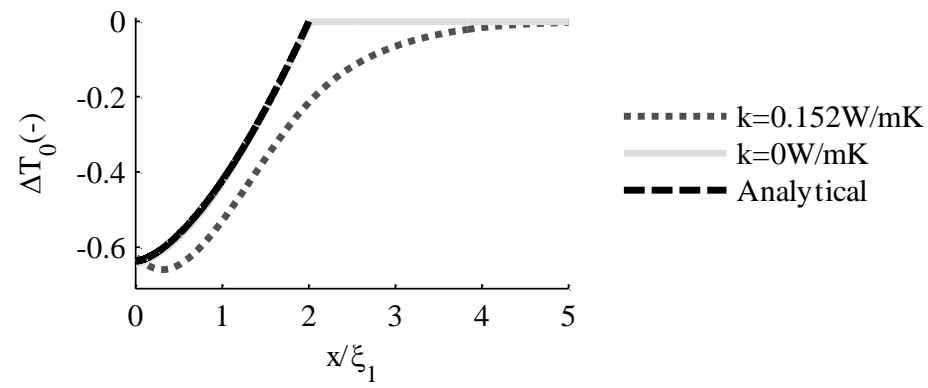

Figure 4.17: Deviation of the mean temperature normalized with the adiabatic temperature amplitude and plotted over the dimensionless $x$-coordinate.

\subsection{Calculation of thermoacoustic functions ${ }^{1}$}

In this chapter the method for the calculation of the thermoacoustic functions is validated by investigating a well-known geometry: the circular pore. The model consists of a circular pore with an entrance length on both sides of it such that the wave propagation is adiabatic at the boundaries of the computational domain. The pressure and the velocity are recorded at both extremities of the domain such that the thermoacoustic functions can be calculated following the procedure given in Chapter 2.2.4.3.2.

In Chapter 4.6.1 the cylindrical pore model is defined and the results are discussed in Chapter 4.6.2.

${ }^{1}$ This chapter is published in Bühler et al.; Calculation of thermoacoustic functions with computational fluid dynamics, Proceedings of Meetings on Acoustics, 2013, Vol. 19 


\subsubsection{Cylindrical pore model}

The model consists of a circular pore surrounded by an adiabatic space. The wave enters the domain at the left side through a non-reflecting boundary condition, interacts at the center of the domain with the cylindrical pore and exits the domain at the right side through another non-reflecting boundary condition. The wall of the pore is set to no-slip and isothermal with a wall temperature of $T_{\text {wall }}=300 \mathrm{~K}$. The inlet and outlet length are modeled as an adiabatic duct, with adiabatic slip radial boundary conditions. The boundary conditions of the model are summarized in Figure 4.18.

The pressure and the velocity are recorded two displacement amplitudes away from the axial boundary conditions in order to avoid numeric effects of the non-reflecting boundary condition on the calculation of the thermoacoustic functions. Using the surface averaged values of the pressure and the velocity the thermoacoustic functions can be calculated following the procedure given in Chapter 2.2.4.3.2.

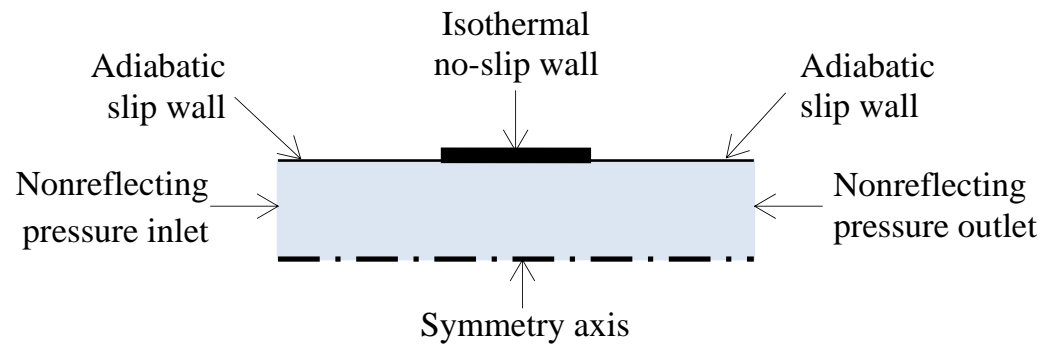

Figure 4.18: Definition of the boundary conditions of the cylindrical pore model.

Four circular pores of radius $R=2 \cdot \delta_{v}$ and different lengths, as defined in Table 4.15 , are numerically investigated. The distance between the point where the data is extracted and the cylindrical pore is $L_{a}=10 \cdot \delta_{v}$. This allows the modeling of the adiabatic wave propagation outside of the pore and the pore end effects.

Table 4.15: Pore length $L$ for the different numerical experiments.

\begin{tabular}{lc}
\hline & Pore length \\
\hline Experiment 1 & $10 \cdot \delta_{v}$ \\
Experiment 2 & $20 \cdot \delta_{v}$ \\
Experiment 3 & $30 \cdot \delta_{v}$ \\
Experiment 4 & $100 \cdot \delta_{v}$ \\
\hline
\end{tabular}

The acoustic wave entering the domain at the left has a frequency of $f=100 \mathrm{~Hz}$ and an amplitude of $p_{1}=100 \mathrm{~Pa}$. The low pressure amplitude is selected as this is a valida- 
tion case and the assumptions made in order to derive the analytical solution are fulfilled for low amplitudes. Furthermore, this allows reduces the computational domain as the particle displacement is small. In the present case, the particle displacement amplitude is on the order of the magnitude of the penetration depth.

No turbulence model is applied as according to Olson and Swift [8], the flow field is laminar for a dimensionless radius of $R / \delta_{v}=2$ and a Reynolds number of $R e \approx 12$.

At $t=0$ the domain is initialized with zero velocity, zero relative pressure and a temperature $T_{0}=300 \mathrm{~K}$. In total, two periods are simulated leading to a total simulation time of $t=0.02 \mathrm{~s}$. The first period is necessary for the transient effects to build up, while the second is only used for post-processing.

The full time dependent compressible Navier Stokes equations are solved with the optimal numerical options derived in Chapter 4.2 and summarized in Table 4.6. As gradients are much higher in the radial direction than in the axial direction, a rectangular mesh is chosen. This allows for a much finer grid in the radial direction compared to the axial direction. The mesh in the axial direction is uniform with three elements per viscous penetration depth, corresponding to 49000 elements per wavelength. The mesh in radial direction consists of 25 elements growing geometrically by a factor of $q=1.2$ from the wall to the symmetry boundary. The time step size is limited by the non-reflecting boundary condition and the discretization in axial direction. According to these limitations the time step size is set to $\Delta t=5 \cdot 10^{-7} \mathrm{~s}$, which corresponds to 20000 time steps per period. Pressure and velocity are exported every 40 time steps to obtain 500 measurement points per wave period.

\subsubsection{Results}

In a preliminary study the wall of the cylindrical pore is set to adiabatic slip. The resulting characteristic impedance $Z_{a}$ and wave number $k_{a}$ can be estimated from the simulation using the procedure of Udea et. al. described in Chapter 2.2.4.3.2. as follows:

$$
\begin{gathered}
Z_{a}=(166.7+0.0674 i) \frac{\mathrm{kg}}{\mathrm{m}^{2} \mathrm{~s}} \\
k_{a}=\left(0.6145+5.64 \cdot 10^{-5} i\right) \frac{1}{\mathrm{~m}}
\end{gathered}
$$

This corresponds to adiabatic wave propagation and can be compared to the analytical values. The deviation between both is less than $1 \%$.

From the characteristic impedance $Z_{a}$ and wave number $k_{a}$ the corresponding thermoacoustic functions can be computed:

$$
\begin{gathered}
f_{\kappa}=-1.38 \cdot 10^{-2}-4.64 \cdot 10^{-4} i \\
f_{v}=2.69 \cdot 10^{-3}+4.95 \cdot 10^{-4} i
\end{gathered}
$$

The theoretical thermoacoustic functions for adiabatic wave propagation are zero. Knowing that the thermoacoustic functions range between zero and one, it can be con- 
cluded that the simulations have good agreement with the analytical value. The largest deviations occur in the real part of the thermal thermoacoustic function. Using the notation used in Swift [6], this deviation can be interpreted as an underestimation of the compliance $c$ in the simulations:

$$
c=\frac{A}{\gamma p_{0}}\left(1+(\gamma-1) \operatorname{Re}\left(f_{\kappa}\right)\right)
$$

The wave propagation characteristics obtained from the preliminary study are used in the following to model the adiabatic wave propagation in the method of Udea et. al. This allows an estimation of the thermoacoustic functions of the cylindrical pore to be made. The values obtained from the simulations are summarized in Table 4.16 and compared to the analytical values calculated from Equation (B.4):

$$
\begin{aligned}
& f_{v}=0.537-0.369 i \\
& f_{\kappa}=0.678-0.373 i
\end{aligned}
$$

The results for the viscous thermoacoustic function match the analytical solution to $5 \%$, while the results for the thermal thermoacoustic function match to $30 \%$. In both cases the results become continuously better with increasing pore length, $L$. The difference between the simulation and the analytical solution can be explained by the pore end effects. The method from Udea et al. provides averaged values of the viscous and thermal effects over the whole length of the pore. Assuming that the same pore end effects occur in all experiments, they will become of larger influence for shorter pores. A very long pore should converge to the analytical solution, as the analytical solution holds for an infinite pore.

Table 4.16: Computed values of viscous and thermal thermoacoustic functions, separated in real and imaginary part, compared with the analytical solution (Equation (4.8) and (4.9)) for the four different lengths.

\begin{tabular}{ccccccccc}
\hline & \multicolumn{2}{c}{$\boldsymbol{f}_{\boldsymbol{v}}$} & \multicolumn{2}{c}{ Deviation } & \multicolumn{2}{c}{$\boldsymbol{f}_{\boldsymbol{\kappa}}$} & \multicolumn{2}{c}{ Deviation } \\
& $\mathbf{R e}$ & $\mathbf{I m}$ & $\mathbf{R e}$ & $\mathbf{I m}$ & $\mathbf{R e}$ & $\mathbf{I m}$ & $\mathbf{R e}$ & $\mathbf{I m}$ \\
\hline Exp. 1 & 0,5629 & $-0,3748$ & $4,9 \%$ & $1,5 \%$ & 0,6179 & $-0,4806$ & $-8,9 \%$ & $28,9 \%$ \\
Exp. 2 & 0,5493 & $-0,3708$ & $2,3 \%$ & $0,4 \%$ & 0,6356 & $-0,4279$ & $-6,3 \%$ & $14,8 \%$ \\
Exp. 3 & 0,5447 & $-0,3697$ & $1,5 \%$ & $0,1 \%$ & 0,6442 & $-0,4097$ & $-5,0 \%$ & $9,9 \%$ \\
Exp. 4 & 0,5381 & $-0,3678$ & $0,3 \%$ & $-0,4 \%$ & 0,6532 & $-0,3855$ & $-3,7 \%$ & $3,4 \%$ \\
\hline
\end{tabular}

The end effects can be eliminated with the end correction method, as long as the pore end effects are independent of the pore length. This is now verified. Figure 4.19 a) shows the axial velocity normalized with the velocity amplitude at $r=0$, over the $x$ axis, in the 
vicinity of the left pore end. The axial velocities of all four experiments are plotted at four moments in time, separated by a quarter period each. The gray and white area represent the pore and the open space, respectively. The profiles lay closely over each other showing that the same pore end effects occur independent of the total length of the pore. The end correction method can thus be applied to obtain the viscous thermoacoustic function of the inner part.

The same reasoning can be applied to the temperature profile. Figure $4.19 \mathrm{~b}$ ) shows the temperature changes normalized with the adiabatic temperature amplitude at a radial coordinate of $r=0$, over the $x$ axis, in the vicinity of the left pore end. Again, the temperature oscillations of all four experiments are plotted at four moments, separated by a quarter period each. As was the case for the axial velocity, the profiles nearly lay over each other. The only small deviation occurs in the temperature profile of Experiment 1 $\left(L=10 \cdot \delta_{v}\right)$ at $x / \delta_{v}=14 \ldots 15$, which is the center of this pore. In this case the pore is too short and the end effects fill the entire pore. For the other lengths the profiles are the same. It can thus be concluded that the end correction method can be applied to obtain the thermoacoustic functions of the inner part of the pore.

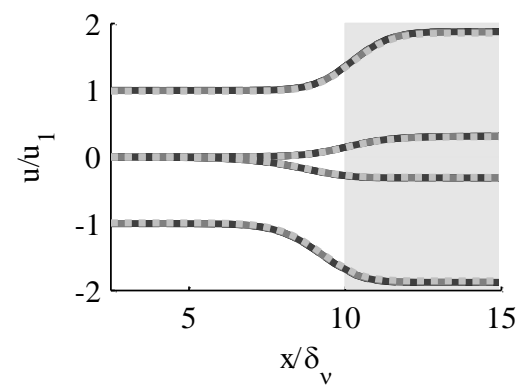

a)

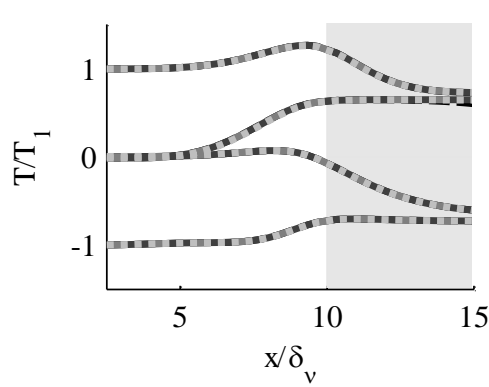

b)

Figure 4.19: a) Axial velocity normalized with the velocity amplitude b) Temperature changes normalized with the adiabatic temperature amplitude at the left of the domain at $r=0$, over the $x$ axis, in the vicinity of the left pore end at four different moments, separated by a quarter period each. The four different pore lengths are shown with a lighter line color indicating a longer pore. The gray and white area represent the pore and the open space domain respectively.

Furthermore, Figure 4.19 a) and Figure 4.19 b) show that the domain of the pore in which the pore end effects occur, is larger for the thermal effects $\left(\sim 5 \delta_{v}\right)$ than for the viscous effects $\left(\sim 2 \delta_{v}\right)$. This is due to the heat pumping effect that occurs at the extremity of the pore, which is investigated in more details in Chapter 5.4. This effect can explain why in Table 4.16 the differences with the analytical solution are larger for the thermal thermoacoustic function compared to the viscous thermoacoustic function. 
In the next step, the end correction method is applied to two simulations with different lengths. This allows subtracting the effects at the extremities of the pore by comparing the two simulations. The two shortest pores, Experiment 1 and 2, are chosen as they are the most limiting for the end correction method. The thermoacoustic functions of the "inner" part of the pore, which are not affected by end effects, are calculated. These two values are then compared with the analytical solutions. The results are summarized in Table 4.17. With this method the difference with the analytical solution can be reduced to less than one percent except for the real part of the thermal thermoacoustic function. The end correction can reduce the deviation of the real part of the thermoacoustic function with the analytical solution, but the deviation still stays at a higher value of approximately $4 \%$. Even if the end correction method is applied to different combinations of the four experiments, the differences stay the same. It can thus be concluded, that this deviation is not caused by the pore length. Further, simulations with pores having a slip boundary instead of a no-slip wall boundary condition exclude viscosity driven streaming as a cause because the deviation occurs there as well. A reduction of the pressure amplitude to $p_{1}=1 \mathrm{~Pa}$ reduces the deviation to roughly $1.5 \%$, possibly due to the reduced convection.

Table 4.17: Computed values of viscous and thermal thermoacoustic functions, separated in real and imaginary part, compared with the analytical solution (Equation (4.8) and (4.9)) for the first two experiments and the estimated value of a pore without end effects computed from the first two experiments.

\begin{tabular}{lcccccccc}
\hline & \multicolumn{2}{c}{$\boldsymbol{f}_{\boldsymbol{v}}$} & \multicolumn{2}{c}{ Deviation } & \multicolumn{2}{c}{$\boldsymbol{f}_{\boldsymbol{\kappa}}$} & \multicolumn{2}{c}{ Deviation } \\
& $\mathbf{R e}$ & $\mathbf{I m}$ & $\mathbf{R e}$ & $\mathbf{I m}$ & $\mathbf{R e}$ & $\mathbf{I m}$ & $\mathbf{R e}$ & $\mathbf{I m}$ \\
\hline Exp. 1 & 0,5629 & $-0,3748$ & $4,9 \%$ & $1,5 \%$ & 0,6179 & $-0,4806$ & $-8,9 \%$ & $28,9 \%$ \\
Exp. 2 & 0,5493 & $-0,3708$ & $2,3 \%$ & $0,4 \%$ & 0,6356 & $-0,4279$ & $-6,3 \%$ & $14,8 \%$ \\
End & & & & & & & & \\
corrected & 0,5355 & $-0,366$ & $-0,2 \%$ & $-0,9 \%$ & 0,6530 & $-0,3754$ & $-3,7 \%$ & $0,7 \%$ \\
\hline
\end{tabular}

In this Chapter the experimental method of Udea et al. is validated for the CFD model of the cylindrical pore. The deviation between the analytical solution and the experiments are due to the end effects as was shown by subtracting them out of the results. After subtraction of the end effects, the deviations stay below 5\%. It can be concluded that the method of Udea et al. predicts correctly the thermoacoustic functions of CFD simulations. 



\section{Numerical experiments and results}

In the previous chapters the implemented boundary conditions were presented and validated against the low reduced frequency approximation. The necessary discretization and the best numerical parameters for the thermoacoustic simulations were derived. With these optimal numerical parameters known, components of a thermoacoustic device are studied in this chapter. The goal of the following four studies is to show that investigating geometric parameters of components detached from the rest of the thermoacoustic device is possible and can lead to a better understanding of the underlying flow phenomena, especially the non-linear effects cannot be neglected.

The first investigated component is the thermal buffer tube. In Chapter 5.1 the streaming patterns inside the thermal buffer tube are revealed and the resulting temperature profiles are given. The second investigated component is the U-bend that feeds back the acoustic wave in a traveling wave device. In the scope of reducing the size of the thermoacoustic devices, the bends gets increasingly sharp, introducing additional losses. In Chapter 5.2 the influence of the geometric parameters of the bend on the flow field are investigated. In the subsequent Chapter 5.3 an experimental method to calculate the thermoacoustic functions is applied to CFD. The method is applied to a reduced model of a stacked screen regenerator and the thermoacoustic functions are given for different geometries of the regenerator model. Finally, in Chapter 5.4 the non-linear entrance effects at a stacked screen regenerator are investigated.

\subsection{Thermal buffer tube}

In this chapter heat transfer is investigated using a CFD model in a large component of a traveling wave thermoacoustic engine: the thermal buffer tube. The goal of the thermal buffer tube in a thermoacoustic engine is to provide thermal insulation between the hot heat exchanger and the secondary ambient heat exchanger while transmitting the acoustic power out of the hot zone [14]. Pulse tube refrigerators have a component with the same functions, which is called the pulse tube. Similar flow fields are to be expected in both, the thermal buffer tube and the pulse tube, as they only differ in sign of the temperature gradient. In the present study a negative temperature gradient in wave propagation is investigated, corresponding to a thermal buffer tube.

An important effect in the thermal buffer tube is steady convection that is induced by the acoustic oscillations. This phenomenon is called acoustic streaming and is an unwanted effect as it can be an acoustic power loss mechanism and a way of wasteful convecting heat between the two heat exchangers [9]. The present study is meant to reveal these streaming patterns that are responsible for the thermal losses in a realistic thermal buffer tube and to show the effect on the temperature distribution inside the thermal buffer tube. 
In the past years, models have been developed in order to describe the effects of streaming in the thermal buffer tube. The streaming induced by the interaction between the acoustic wave and a boundary was first modeled by Lord Rayleigh [79], who gave an analytical description in the case of a standing wave in a waveguide with a constant cross-section. Later, Rott [80] proposed a model for streaming in waveguides with constant cross-section, subject to an axial temperature gradient. The derivation is done from the boundary layer limit assuming a standing wave phasing between pressure and velocity. In their works, Bailliet [81] and Hamillton [82] proposed analytical solutions that could remove the restriction to wide pores.

Lee et al. [83] suggested that slightly tapering the thermal buffer tube would suppress streaming. To demonstrate this, Olson and Swift [84] conducted experiments and developed a model allowing for any wave phasing in a waveguide subject to a temperature gradient and with a variable cross-section. This model is used in this chapter in order to validate the simulations. A more general review on streaming can be found in Boluriaan et al. [85] and $\mathrm{Gu}$ et al. [86] which give a review on streaming in pulse tubes.

In literature another approach applied to reveal the flow field inside the thermal buffer tube is CFD. The approaches can be split into two groups. The first consists of simulations only modeling the thermal buffer tube. In these simulations the thermal buffer tube is modeled as a tube in which an acoustic oscillation is introduced on one side, while the other side of the tube is closed by a wall. He et al. [87] impose the pressure at the left side of the tube and give the streaming field for different taper angles in order to show that an optimal taper angle exists. Aktas et al. [88] impose the velocity at the left side of the tube and show that for smaller radii stable vortices occur while for larger radii the vortices become irregular and complex.

The second group of simulations models the entire device. Simulations of traveling wave engines in a configuration like Backhaus and Swift [14] proposed are rare. Lycklama à Nijeholt et al. [42] conducted CFD simulations of an entire engine, but the numerical mesh used does not fulfill the requirements derived in Chapter 4.1. For this reason the general validity of the results are suspect. More simulations have been carried out on pulse tube refrigerators. In the system level simulations the influence of the thermal buffer tube on the overall efficiency of the refrigerator is investigated. This approach allows for example Zhang et al. [89] to reveal the flow field at the intersection of the thermal buffer tube and the heat exchanger, which creates a swirl. Gu et al. [90] show the influence of a cross-sectional jump at one extremity of the thermal buffer tube. The streaming vortices introduced by the change in cross-section can seriously deteriorate the overall performance of the refrigerator. But streaming does not necessarily reduce the performance of thermoacoustic devices, Antao et al. [91] showed, that streaming can also be beneficial. They revealed that in the case of well-developed streaming flow patterns a thermal buffer zone in the center of the thermal buffer tube occurs and enhances the overall performance. 
Next to streaming the interaction of the heat exchanger with the fluid in the thermal buffer tube plays an important role. This effect was described in Chapter 4.5. A simple explanation of this effect can be found in Swift [6] and a more detailed one in Matveev et al. [76]. The numerical models given in these papers are used in this thesis for validation purposes and will be presented in Chapter 5.1.1.

In this thesis the thermal buffer tube of a traveling wave engine detached from the other components of the thermoacoustic engine will be investigated using CFD. This is possible with the boundary condition that has been implemented in ANSYS Fluent 14 [39]. This boundary condition is presented in Chapter 3.2 and validated in Chapter 4.3. This approach allows extending the analytical solution and the component wise simulations by the effect of the ideal heat exchangers at the extremities of the thermal buffer tube. Furthermore, it is the first simulation that models the thermal buffer tube separated from the other components of a traveling wave thermoacoustic engine.

In the following section both the CFD model and a one-dimensional model are presented. Chapter 5.1.2 gives the results from the CFD simulations while Chapter 5.1.3 gives a general conclusion and recommendations for future work.

\subsubsection{Thermal buffer tube models}

The thermal buffer tube investigated in this chapter has the dimensions of that found in the thermoacoustic engine presented by Backhaus and Swift [9]. The parameters are taken from the DeltaEC model TASHE1.out [7]. The thermal buffer tube is modeled as a cylindrical tube, having a length of $L=0.24 \mathrm{~m}$ and a radius of $R=0.044 \mathrm{~m}$. The wave enters at the left side of the domain where the hot heat exchanger is situated and leaves the domain at the right where the ambient (cold) heat exchanger is situated. The thermal buffer tube is further defined by the fluid parameters taken from the DeltaEC model and summarized in Table 5.1.

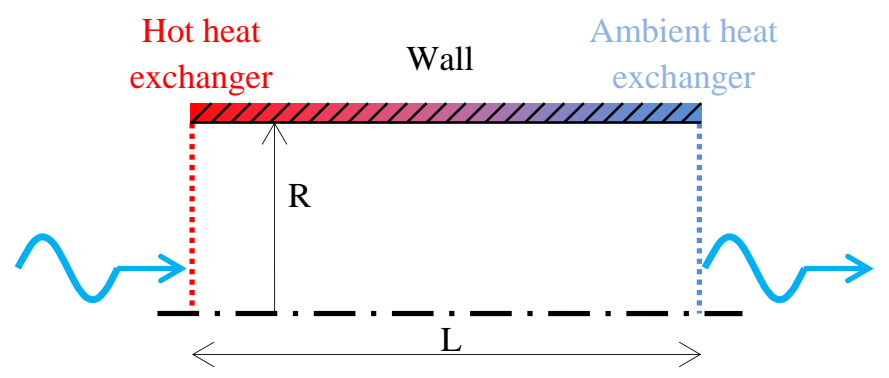

Figure 5.1: Thermal buffer tube model with ideal heat exchangers at the ends and wall with linear temperature gradient. 
Table 5.1: Fluid parameters taken from the DeltaEC model TASH1.out [7].

\begin{tabular}{cc}
\hline Parameter & Variable and value \\
\hline Mean pressure & $p_{0}=31 \cdot 10^{5} \mathrm{~Pa}$ \\
Temperature hot heat exchanger & $T_{H}=825 \mathrm{~K}$ \\
Temperature secondary ambient & $T_{C}=325 \mathrm{~K}$ \\
heat exchanger & $f=84.12 \mathrm{~Hz}$ \\
Frequency & $f$
\end{tabular}

\subsubsection{CFD model}

In this chapter the CFD model used to investigate the thermal buffer tube is described. The first part of the chapter gives the physical models, while the second part defines the numeric discretization of the domain.

The thermal buffer tube is filled with helium. Since the temperature dependency is an important cause of streaming and because neglecting this dependency would lead to significant errors [84], helium is modeled as an ideal gas with a temperature dependent viscosity and heat conduction (see Appendix E.1). The mean pressure inside the thermal buffer tube is set to $p_{0}=31 \cdot 10^{5} \mathrm{~Pa}$. The wall boundary condition is modeled in different ways in order to reveal the influence of the thermal and viscous boundaries on streaming and energy losses. Therefore, the two thermal and two viscous boundaries are combined for a total of four simulations. The thermal boundary condition is either adiabatic or it is assumed to have a linearly distributed, but time independent wall temperature. The wall temperature between the two heat exchangers is chosen as the thermal effusivity and the heat conduction of the stainless steel wall are three orders of magnitude higher than that of helium. The viscous wall boundary condition is assumed to be either slip or no-slip. This means that no surface roughness is taken into account, corresponding to the assumption that the surface roughness is much smaller than the thermal and viscous penetration depth. This is an approximation which can be done as the thermal buffer tubes are generally polished in order to minimize the heat transfer due to the surface roughness [6].

The acoustic wave enters at the left of the domain by applying a time dependent mass flux with an amplitude such that the drive ratio is $D r=1 \%$. The wave has a frequency of $f=84 \mathrm{~Hz}$. In this model a mass flux inlet is chosen instead of the non-reflecting boundary condition presented in Chapter 3.2. This is due to the fact that in the present model the non-reflecting boundary conditions on both extremities of the domain would lead to an unwanted velocity drift, introducing large convection over the boundaries (see Appendix 
$\mathrm{K}$ for a detailed study). The drifting due to the boundary condition is higher than in the boundary condition validation case in Chapter 3.2.3, because of the temperature gradient across the thermal buffer tube. These temperature changes introduce small errors in the wave propagation that accumulate and lead to drifting. Because of the drifting, the mean velocity becomes so high that the convection through the thermal buffer tube would dominate. This is not realistic as in real engines the mean flow through the entire feedback loop would be canceled by a jet pump [14]. This is the reason for choosing a fully reflective mass flux inlet at the left to introduce the wave.

The wave leaves the domain at the right through a non-reflecting boundary condition. The propagation constant $\Gamma$, which is needed for the non-reflecting boundary condition can be calculated with equation (2.71) using the kind of polytropic constant $n$ defined in (2.72) and the thermoacoustic function corresponding to the chosen wall boundary condition (see Appendix B). The temperature of the fluid entering the domain is set to the temperature of the heat exchanger modeled. This corresponds to a temperature at the left of $T_{L}=825 \mathrm{~K}$ and at the right of $T_{R}=325 \mathrm{~K}$.

Turbulence can be another important effect in the thermal buffer tube. According to Olson and Swift [84] the regime inside of the present thermal buffer tube can be estimated from the Reynolds number and the ratio of the radius to the viscous penetration depth. For the present model both parameters are calculated in the center of the thermal buffer [84]:

$$
\begin{gathered}
R e=\frac{2\left\langle u_{1}\right\rangle R \rho_{0}}{\mu_{0}}=7.3 \cdot 10^{4} \\
\frac{R}{\delta_{v}}=210
\end{gathered}
$$

According to Olson and Swift [84] the flow field is in a weakly turbulent regime for the present simulations. In this regime the turbulence is generated outside of the viscous penetration depth, leaving the velocity close to the wall nearly the same as it would be for laminar flow [84]. In the present study the most important effects such as streaming are induced in the vicinity of the boundary layer. For this reason it can be expected that the results are qualitatively right, hence no turbulence model is implemented.

The fluid domain is initialized by running a preliminary steady state simulation without acoustic perturbation, in order to get the temperature field. The velocity is set to zero in the whole domain. The thermal initialization of the domain is important because the heat transfer coefficient is temperature dependent and because the time scale of the acoustic wave is smaller than that of the heat conduction. These different time scales will be discussed in more details in the results Section 5.1.2.1.

In the preceding part the choice and the motivation for the physical models to simulate the thermal buffer tube have been given. The resulting equations are discretized and the choice of the numerical parameters is given in the subsequent part. 
The thermal buffer tube is discretized with a quadrilateral mesh. This allows using a different mesh resolution in the radial and the axial direction. The mesh in the radial direction is chosen such that there are at least 10 elements in the extended boundary layer $\pi \delta_{v}$, with a maximal growing factor of $q=1.2$ (see Chapter 4.1). With this requirement and using the penetration depth at the secondary ambient heat exchanger, $\delta_{v}=1.3$. $10^{-4} \mathrm{~m}$, as it is the smallest at that location, the size of the first cell $h_{0}$ can be calculated as follows:

$$
h_{0}=\frac{\delta_{v} \pi(r-1)}{q^{10}-1}=1.57 \cdot 10^{-5} \mathrm{~m}
$$

In the present case a large channel is investigated. In order to avoid elements at the axis which are to large, the growing factor is set to 1.1. This increases the number of elements in the boundary layer. From this the total number of elements in the radial direction can be calculated as:

$$
n=\frac{\ln \left(\frac{R(q-1)}{h_{0}}+1\right)}{\ln (q)}=60
$$

In the axial direction a uniform mesh with 300 elements in the wave propagation direction is chosen. This corresponds to more than 15000 elements per wavelength, which is large enough according to the mesh study presented in Appendix L. The time is discretized with a time step of $\Delta t=1.5 \cdot 10^{-5} \mathrm{~s}$. This corresponds to 790 time steps per period. The simulation time is chosen such that 300 periods are simulated in order to obtain a steady periodic solution. The further discretization options are the same as the ones given in Table 4.6. The typical calculation time for a simulation is about 20 days on a single i7$2600 \mathrm{CPU}$. The simulations take so long because 300 periods have to be simulated in order to reach a steady periodic state. In the present case, parallelization does not have a positive effect as the simulations have a low number of spatial mesh points but a large number of time steps, which leads to a high additional overhead.

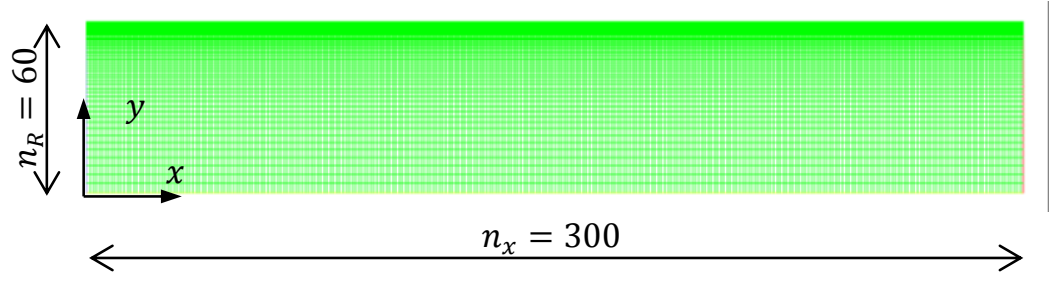

Figure 5.2: Numerical mesh of the thermal buffer tube model. 


\subsubsection{One-dimensional model}

The CFD simulations are compared to a one-dimensional numerical model, where only the one-dimensional heat equation is solved. This means that two-dimensional effects like acoustic streaming are not taken into account in the one-dimensional model. The numerical model follows the approach of Matveev et al. [76] and Berson et al. [92], who compared their numerical results to experimental results and showed good agreement close to the heat exchangers. These numerical models extend the analytical model presented in Chapter 4.5.1.1, by incorporating the heat conduction in the axial direction. While Matveev et al. [76] solved the temperature equation in the Lagrangian point of view, Berson et al. [92] used the dimensionless temperature equation in the Eulerian coordinates. In this thesis the second point of view is chosen and the following temperature equation is solved assuming that the changes in fluid properties are small:

$$
\frac{\partial T}{\partial t}=\frac{\gamma-1}{\gamma} \frac{T}{p} \frac{\partial p}{\partial t}-u \frac{\partial T}{\partial x}+\frac{\gamma-1}{\gamma} k \frac{T}{p}\left(\frac{\partial^{2} T}{\partial x^{2}}\right)+K\left(T_{H X}-T\right)
$$

where the factor $K$ is the heat transfer coefficient between the heat exchanger and the fluid. If a temperature gradient is applied, the heat transfer coefficient inside the thermal buffer tube can be calculated from the geometry dependent thermoacoustic functions as follows [93]:

$$
K=\left|-i \omega\left(1-\frac{\left(1-f_{v}\right)(1-\operatorname{Pr})}{\left(1-f_{\kappa}\right)-\operatorname{Pr}\left(1-f_{\kappa}\right)}\right)\right|
$$

The thermoacoustic functions are calculated assuming the boundary layer approximation as the radius is large compared to the penetration depths (see also Chapter 2.2.4.1). The pressure and the velocity are imposed assuming both a traveling wave and no spatial variations inside of the domain:

$$
\begin{gathered}
p(t)=p_{0}+p_{1} \sin (\omega t) \\
u(t)=u_{1} \sin (\omega t)
\end{gathered}
$$

The temperatures at the left and right of the domain are set to the constant value of the heat exchanger in the case of an inflow and to no-flux $\partial T / \partial x=0$ in the case of the fluid leaving the domain. The domain is initialized by solving the steady state temperature equation, as the heat conduction is temperature dependent ${ }^{1}$. The resulting temperature profile is not linear, especially when the boundary is assumed adiabatic. The simulated time is the same as for the CFD model and the last two periods of both models are compared.

The temperature equation is solved with the MATLAB function pdepe(), which solves initial-boundary problems for parabolic partial differential equations in one dimension. More details about the implementation in MATLAB can be found in Appendix $\mathrm{H}$.

\footnotetext{
${ }^{1}$ Note that in Equation (5.5) it is assumed that the changes in heat conductivity are small.
} 
Each of the simulations takes about 30 minutes, which is considerably less than the full CFD model.

\subsubsection{Results and discussion}

In this chapter the results of the thermal buffer tube models are presented. In a preliminary step the periodicity of the simulation is investigated in Chapter 5.1.2.1 and some remarks about the relevant time scales are given. Chapter 5.1.2.2 investigates the streaming inside the thermal buffer tube for different wall boundary conditions. The influence on the mean temperature distribution is revealed in Chapter 5.1.2.3. Finally, in Chapter 5.1.2.4 the different heat loss mechanisms in the thermal buffer tube are investigated.

\subsubsection{Periodicity of the simulations}

The simulations are run for 300 periods, which is equivalent to a total simulation time of $t=3.57 \mathrm{~s}$. This time is selected so that the initial transient behavior due to the acoustic field is damped out. However, the obtained flow field is not yet steady periodic, as the time scale of the conductive effects is much larger than that of the acoustic effects. To demonstrate this, the Fourier number $F o$ is calculated at both ends of the thermal buffer tube using the total simulation time [94]:

$$
\begin{aligned}
F o & =\frac{k t}{\rho c_{p} L^{2}} \\
F o_{H} & \approx 2.1 \cdot 10^{-3} \\
F o_{C} & \approx 4.2 \cdot 10^{-4}
\end{aligned}
$$

Both values of the Fourier number are clearly lower than one, which shows that the temperature changes due to conduction are small on the scale of the simulation time.

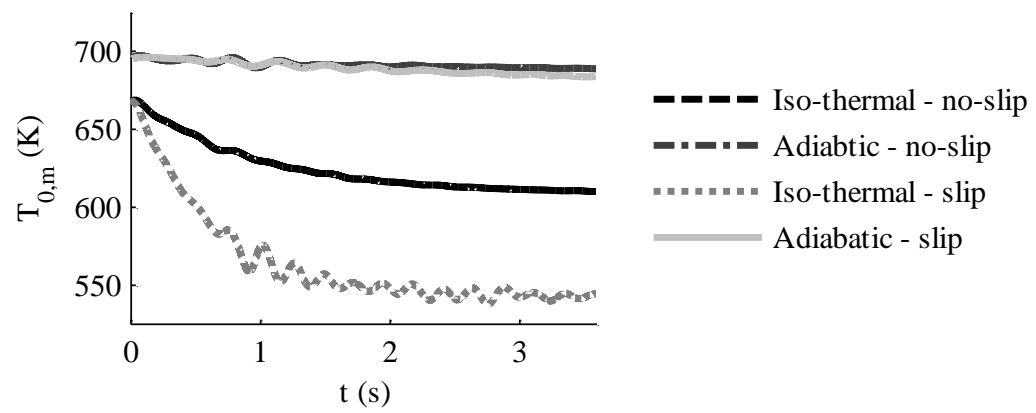

Figure 5.3: Moving average of the temperature over time at the position $x=L / 2$, $R=0$ for the four different combinations of wall boundary conditions, averaging interval is one period.

Figure 5.3 shows the moving average of the temperature inside the domain over time. The average interval is taken over one period. It can be seen that after 300 periods only 
small changes over time occur. These small changes are due to the conductive effects inside the thermal buffer tube. As the timescale for the conductive effects is considerably higher and only small changes in mean temperature occur, it is chosen to investigate the thermal buffer tube after 300 periods.

In addition to the physical effects, there are also some numerical effects that act on the periodicity. Due to the boundary conditions chosen (inlet: mass flow inlet; outlet: non-reflecting) the mean pressure is not fixed. The non-reflecting boundary condition accumulates errors, which leads to pressure drift in the whole domain. This drift is small compared to the mean pressure, thus it has less impact on the flow field inside the thermal buffer tube compared to a non-reflecting pressure inlet boundary condition. Figure 5.4 shows the moving average of the relative pressure at the exit of the domain for four different combinations of wall boundary conditions. The moving average is taken over one period. For all simulations except the case with adiabatic slip walls, the mean pressure drifts to a constant value. In the case of the adiabatic slip wall boundary condition, no dissipation at the wall occurs and the pressure drifts even further after 300 periods. This means that no steady periodic state is reached. In the other cases equilibrium is reached due to the additional physical damping, which limits the numerical drift.

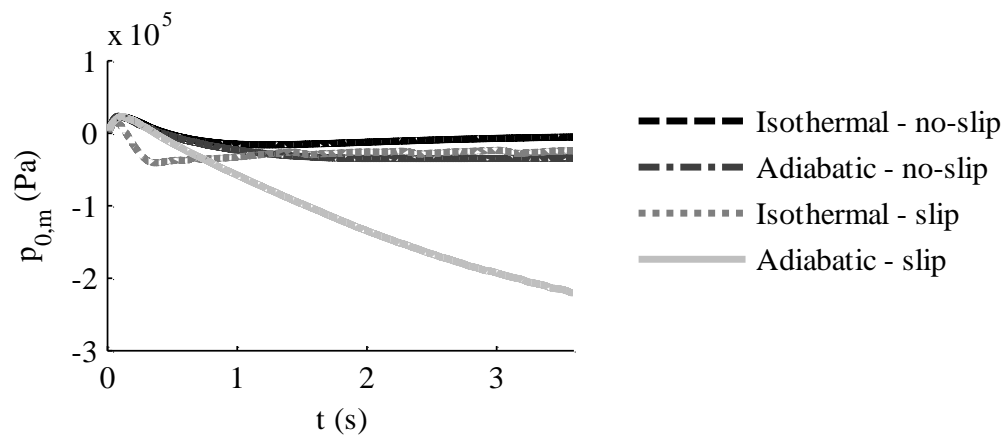

Figure 5.4: Moving average of the relative pressure over time at the position $x=L / 2$, $R=0$ for the four different combinations of wall boundary conditions, averaging interval is one period.

\subsubsection{Acoustic streaming}

The aim of the thermal buffer tube is to transfer acoustic power while insulating the hot heat exchanger from the ambient heat exchanger. For this reason convection of heat due to acoustic streaming is an unwanted effect as it reduces the efficiency of the engine [9]. For this reason mass streaming inside the thermal buffer tube is investigated by looking at the streaming velocity $u_{2}$ (or time average mass transport velocity [88]): 


$$
u_{2}(x)=\frac{\overline{u(\vec{x}, t) \rho(\vec{x}, t)}}{\overline{\rho(\vec{x}, t)}}
$$

In general, boundary layer induced streaming patterns can be differentiated in inner and outer streaming, which corresponds to streaming inside and outside of the boundary layer respectively [85]. In Figure 5.5 the outer streaming is visualized with a streamline plot of the streaming velocity $u_{2}$. Streaming inside the boundary layer is not resolved in the figure and will be shown later. The results from four different combinations of boundary conditions are shown in order to reveal the respective influence of the thermal and viscous boundary.

In Figure 5.5 two effects can be differentiated: the interaction of the acoustic inlet and outlet with the wall and the interaction of the acoustic wave with the wall boundary layer. Both effects are spatially separated: while the effects due to the acoustic boundary extend roughly three displacement amplitudes $\xi_{1}$ from the boundary (red dotted line), the interaction of the acoustic wave with the wall can be seen in the middle part. First, the interaction of the acoustic boundary with the wall is described. The resulting streaming is especially pronounced when the viscous effects at the wall are taken into account, corresponding to the no-slip boundary condition. Figure 5.5 a) and b) show a large recirculation zone outside of the boundary layer for small values of $x$. This recirculation zone is caused by the mass flux boundary condition which imposes a uniform mass flux value over the inlet radius. Due to this, an asymmetry occurs close to the boundary when the flow interacts with the wall. When the flow enters the domain, no boundary layer is developed and the fluid has the heat exchanger temperature. However, when the fluid exits the domain it has built up a viscous and thermal boundary layer. This means that when the fluid enters the domain the viscous boundary layer has to develop. This forces the fluid in direction of smaller radial coordinates, as the mass flow is uniform. This asymmetry leads to the recirculation zones that can be seen for small values of $x$, in the case of no-slip wall boundary conditions in the numerical simulation. In the physical engine this effect could also occur, as the regenerator and heat exchanger act as flow straighteners. But the effect would be reduced because streaming inside the regenerator and heat exchanger could be induced. That streaming inside of the regenerator is possible was shown by a stability analysis carried out by So et al. [95].

The acoustic boundary condition at the right of the numerical domain allows a mass flow over the boundary as only the pressure is imposed. This lead to a considerable recirculation zone over the boundary in the case of the isothermal slip wall boundary condition shown in Figure $5.5 \mathrm{c}$ ). As the temperature of the incoming fluid is uniform over the radius, the same boundary effects as discussed above induce the large recirculation zone over the boundary. An inner and an outer streaming vortex appear. For the other cases no clear vortex structure occurs. 
a)

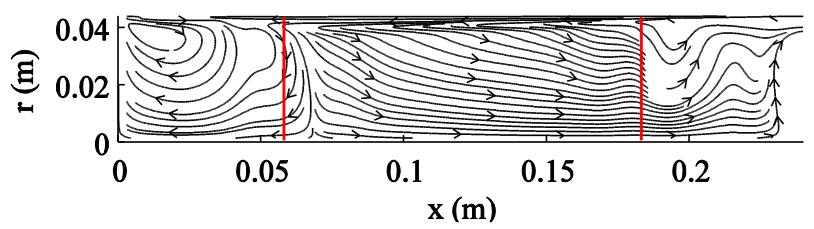

b)

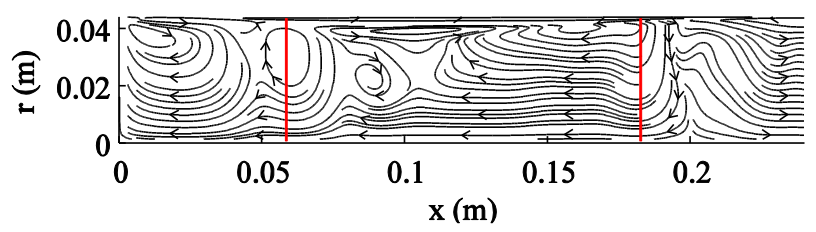

c)

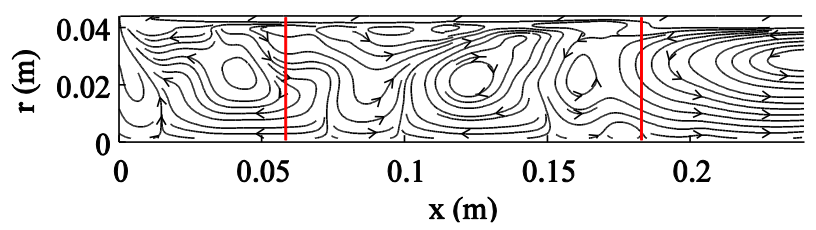

d)

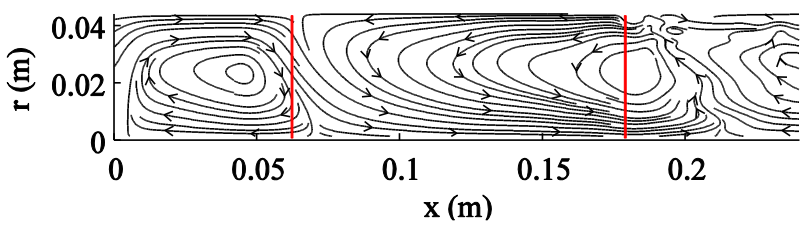

Figure 5.5: Streaming velocity streamlines for the four different boundary conditions: a) isothermal no-slip; b) adiabatic no-slip; c) isothermal slip; d) adiabatic slip; Red lines indicate three displacement amplitudes $\xi_{1}$ from the left and right boundaries

The effect of streaming induced from the acoustic boundary condition extends roughly three displacement amplitudes $\xi_{1}$ inside the domain at both ends. Afterwards, a different structure occurs for all wall boundary conditions. This is surprising as one would expect that these effects only extend two displacement amplitudes from the boundary as gas parcels further away never become in contact with the acoustic boundary. One possible reason is that the boundary layer needs longer than two displacement amplitudes to build up.

In the previous, the outer streaming due to the interaction of the acoustic boundary with the wall was investigated. In the following the remaining part at the center of the thermal buffer tube is investigated. This streaming is created by the interaction of the acoustic wave with the wall boundary. For the isothermal no-slip boundary condition a well-defined flow structure can be seen, which is shown in Figure 5.5 a), representing a pair of streaming vortices. One vortex inside the boundary layer not visible in this figure 
and one outside of the boundary layer. This confirms the approach chosen by Matveev et al. [96], to model only the domain outside of the boundary layer and to incorporate the boundary layer effects with a wall velocity. Only one large vortex structure occurs in the case of the adiabatic slip boundary condition, but the magnitude of the streaming velocity is approximately two orders of magnitude smaller than in all other cases. For the other two combinations of wall boundary conditions smaller vortex structures occur. However, the structures are more irregular.

Next to the outer streaming discussed previously, the inner streaming can be investigated with CFD simulations. Figure 5.6 shows the streaming velocity $u_{2}$ close to the wall for the four different wall boundary conditions. The dotted gray line and the dashed gray line show the viscous and the thermal boundary layer thickness, respectively. In all cases with a boundary layer, the streaming velocity is positive inside the boundary layer and negative outside of the boundary layer. This can be explained with the boundary layer thickness that varies with the length of the tube (see gray lines in Figure 5.6). If the fluid moves in positive $x$-direction, the boundary layer thickness decreases and the fluid experiences less viscous drag, the fluid comes closer to the wall. However, when the fluid moves in negative $x$-direction the penetration depth increases and the fluid is deviated away from the wall [84]. This asymmetry leads to the shown streaming patterns close to the boundary layer.

Figure 5.7 shows the streaming velocity $u_{2}$ over the radius at the center of the tube and for large radial values. The boundary layer induced streaming can be clearly seen by the change of sign of the streaming velocity. The largest velocities are reached for the isothermal slip wall boundary condition at the wall. The two cases with a no-slip boundary have a similar order of streaming. In the case of an adiabatic slip wall the effects are the smallest. From this it can be conclude that the streaming is indeed induced by the wall boundary layers and that the thermal boundary layer induces a larger amount of streaming compared to the viscous boundary. Furthermore, the viscous boundary layer reduces the effect of the streaming induced by the thermal boundary condition, as the viscous boundary layer insulates the wall from the fluid. 
a)

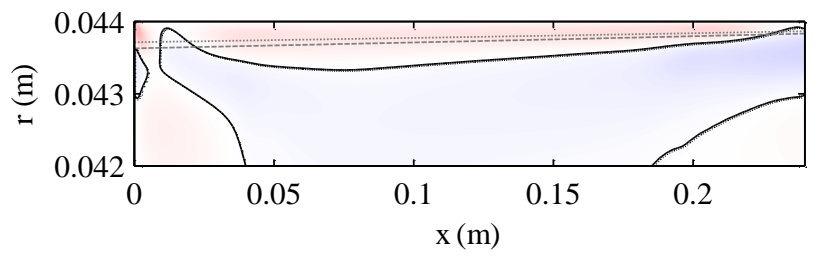

b)

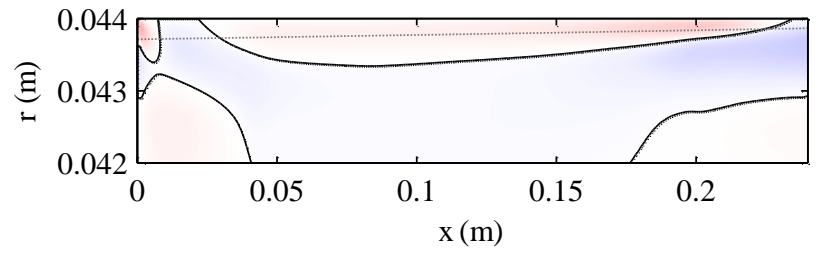

c)

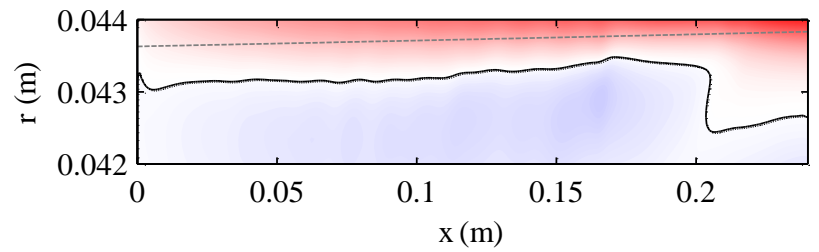

d)
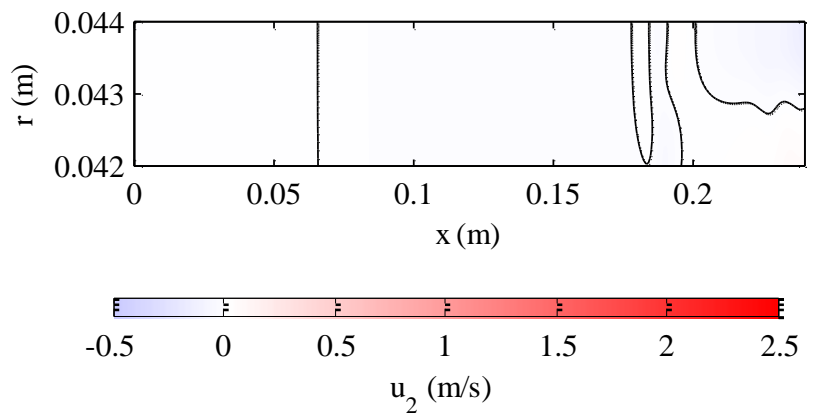

Figure 5.6: Streaming velocity close to the wall of a thermal buffer tube in the case of four different wall boundary conditions: a) isothermal no-slip; b) adiabatic no-slip; c) isothermal slip; d) adiabatic slip. Black lines: zero streaming velocity; dotted gray line: viscous boundary layer; dashed gray line: thermal penetration depth. (figure not to scale). 


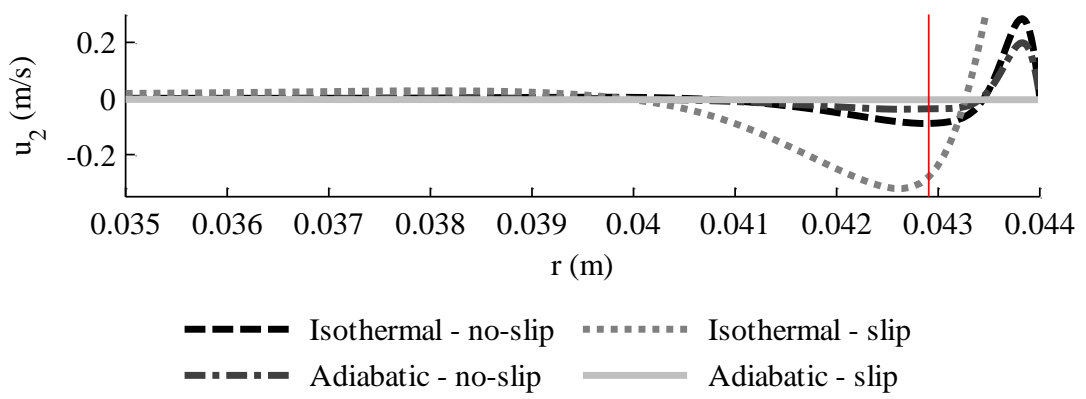

Figure 5.7: Streaming velocity at the center of the thermal buffer tube over the radius, for large radial coordinates and four different wall boundary conditions. Red line indicates outer edge of the boundary layer as defined by Olson and Swift [84].

In order to validate the simulations, the streaming values are compared with the model derived by Olsen and Swift [84]. They derive a theory that predicts the streaming velocity at the outer edge of the boundary layer $\left(R-r \gg \delta_{v}\right.$ and $R-r \gg \delta_{\kappa}$, yet $R-r \ll$ $R$ ). This velocity can be calculated in the case of a cylindrical thermal buffer tube as follows [84]:

$$
\begin{aligned}
u_{2}= & -\frac{3}{4 \omega} \operatorname{Re}\left((1-i)\left\langle u_{1}\right\rangle \omega \frac{p_{1}}{\gamma p_{m}}\right) \\
& +\frac{1}{4 \omega T_{m}} \frac{\partial T_{m}}{\partial x} \frac{(1-b)(1-\sqrt{\operatorname{Pr}})}{(1+\sqrt{\operatorname{Pr}})(1+\operatorname{Pr})}\left\langle u_{1}\right\rangle\left\langle\tilde{u}_{1}\right\rangle \\
& +\frac{1}{2 \gamma p_{m}} \operatorname{Re}\left(\left(\frac{(\gamma-1)\left(1-b \operatorname{Pr}^{2}+i \operatorname{Pr}^{\frac{2}{3}}(1-b)\right)}{\operatorname{Pr}(1+\operatorname{Pr})}-1\right) p_{1}\left\langle\tilde{u}_{1}\right\rangle\right)
\end{aligned}
$$

For the derivation, the viscosity is assumed to vary as a power law of the temperature, with the exponent $b \approx 0.7$. For the numerical evaluation a pure traveling wave is assumed with a pressure amplitude of $p_{1}=31000 \mathrm{~Pa}$ and a surface averaged velocity amplitude of $\left\langle u_{1}\right\rangle=10 \mathrm{~m} / \mathrm{s}$. It follows:

$$
u_{2}=-0.0784 \frac{\mathrm{m}}{\mathrm{s}}
$$

In the CFD simulation, which has the same no-slip and isothermal boundary conditions as the model of Olson and Swift, the minimal value at the outer edge of the boundary layer is (see also Figure 5.7 vertical red line):

$$
u_{2, \min , C F D}=-0.0890 \frac{\mathrm{m}}{\mathrm{s}}
$$

This shows that the CFD simulation can predict the order of magnitude and the direction of the streaming velocity induced by the boundary layer correctly. Deviation might 
be caused by the interaction of the acoustic boundary conditions with the wall boundary conditions at the extremities of the thermal buffer tube.

In the following chapter the influence of the streaming patterns on the mean temperature profile is shown.

\subsubsection{Temperature distribution}

The aforementioned streaming effects have an important influence on the mean temperature profile inside the thermal buffer tube. The temperature profile itself is an important characteristic of the thermal buffer tube as it defines heat losses and acoustic properties. In this chapter the mean temperature profiles inside the thermal buffer tube are given and the consequences of the temperature changes on the acoustic properties are shown.

Figure 5.8 shows the cross-sectional and time averaged temperature over the axial direction in the thermal buffer tube. For guidance the thin black horizontal lines indicate the imposed temperature of the heat exchangers at the ends of the thermal buffer tube and the linear profile between both of them, which is imposed at the wall in the isothermal cases. The other lines show the simulation results for the four different wall boundary conditions.

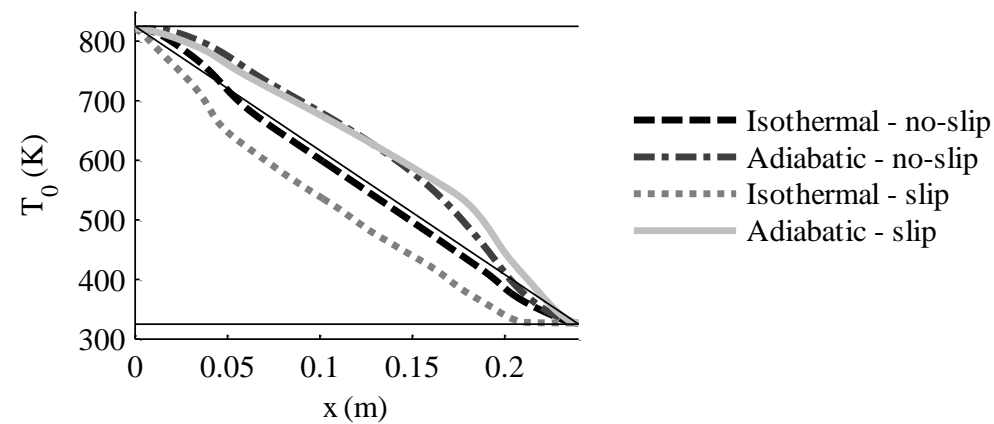

Figure 5.8: $\quad$ Cross sectional and time averaged temperature from CFD over the length $x$ of the thermal buffer tube for four different wall boundary conditions. The thin black lines indicate the temperature of the heat exchangers and the linear profile between them.

The thermal effects of the wall have a large influence on the mean temperature profiles inside the thermal buffer tube. The profiles having an isothermal boundary condition can be clearly differentiated from the profiles with an adiabatic boundary condition. When an adiabatic wall boundary condition is used, no heat is exchanged with the wall. 
Instead, the mean temperature profile inside the thermal buffer tube is defined by the initialization and the first displacement ${ }^{1}$, as will be shown in the following.

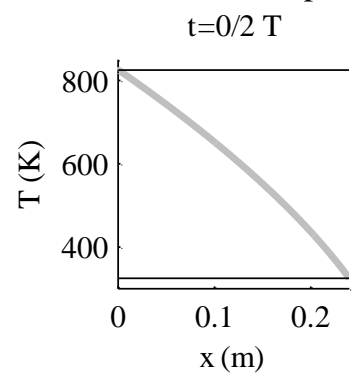

a)

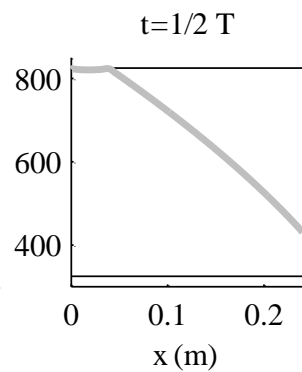

b)

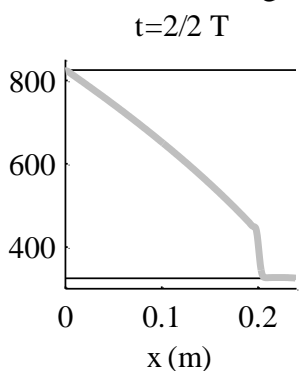

c)

Figure 5.9: Instantaneous area averaged temperature over the $x$-coordinate for the first period at three different time steps spaced by a half period each: a) initial temperature distribution at $t=0 / 2 T ; \mathrm{b}$ ) temperature distribution when fluid reaches the rightmost position at $t=1 / 2 T$; c) temperature distribution when fluid reaches leftmost position at $t=2 / 2 T$. Data from the case with adiabatic slip wall boundary conditions. Thin black lines indicate the heat exchanger temperatures.

Figure 5.9 shows the instantaneous temperature profile inside the thermal buffer tube at the three time instances of the first period where the velocity is zero. In Figure 5.9 a) the initial temperature distribution at $t=0 / 2 T$ is given. Due to the adiabatic wall and the temperature dependent thermal conductivity the profile is not linear. During the following half period the fluid moves to the right, this means that the fluid close to the right heat exchanger is convected into the heat exchanger. The resulting temperature profile at $t=1 / 2 T$, just before the flow reverses, is shown in Figure $5.9 \mathrm{~b}$ ). In the following half period the fluid exits the right heat exchanger with the temperature of the heat exchanger. The linear profile is "cut", as can be seen in Figure 5.9 c). At the hot heat exchanger the fluid is first displaced to the right such that the fluid with a temperature of the heat exchanger enters the domain. In the following half period the fluid with the heat exchanger temperature is convected back. At the hot heat exchanger the linear profile is unchanged after one period. Averaging the temperature over time at every $x$-location leads to a higher temperature than the linear profile inside the domain. Due to the "cut" temperature profile at the cold heat exchanger, a larger gradient occurs at the cold heat exchanger than at the hot heat exchanger. The resulting time averaged temperature profile from the simulations for adiabatic wall boundary condition can be seen in Figure 5.8. During the next periods the profile only changes slightly from the profiles shown in Figure 5.9 b) and c)

${ }^{1}$ Note that conduction over the whole length takes place in a different time scale; Equations (5.10) and (5.11). 
as heat conduction takes place in another time scale than the acoustic one, as was shown with Equations (5.10) and (5.11).

In the case of an isothermal wall boundary condition, the same convective effects at the extremities of the thermal buffer tube occur similar to the cases with adiabatic wall boundary conditions. However, in this case the wall of the thermal buffer tube also affects the average temperature. The interaction is especially large as additional mixing occurs due to streaming. The small over- and undershoot still occurring at the extremities of the thermal buffer tube in Figure 5.8 can be explained with the heat pumping effect that takes place due to the interaction of gas parcels with the ideal heat exchanger. This is the same effect as the one used for the validation of the ideal heat exchanger boundary condition in Chapter 4.5 which extends over two displacement amplitudes $2 \cdot \xi_{1} \approx$ $0.041 \mathrm{~m}$.

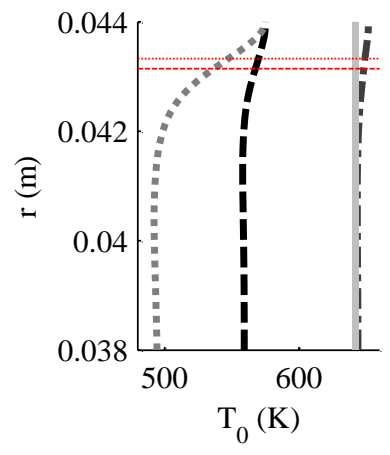

a)

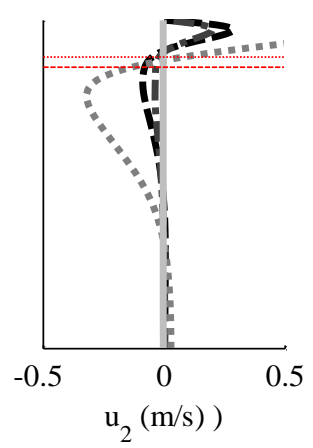

b)

Figure 5.10: a) Mean temperature and b) streaming velocity over the radius (close to boundary) at the center of the tube $(x=L / 2)$ for four different wall boundary conditions. Red dotted line represents the extended viscous boundary layer and the red dashed line represents the extended thermal boundary layer (see Chapter 4.1.2.1)

In the case of isothermal wall boundary conditions, the temperature profile is linear in the center of the thermal buffer tube opposed to the curved profile in the adiabatic case. This is due to the linear isothermal wall boundary condition that influences the temperature profile inside of the thermal buffer tube. Even though the profile is linear in the isothermal no-slip case, the profile is shifted towards smaller $x$-values in the CFD simulations. The shift is a consequence of the streaming that occurs in the vicinity of the boundary layer and can be explained with Figure 5.10 where a) shows the mean temperature and b) the streaming velocity over the radius. The dotted and dashed red lines represent the extended viscous and thermal boundary layer respectively, as defined in Chapter 4.1.2.1. Fluid parcels inside of the thermal boundary layer $\left(r>R-\pi \delta_{\kappa}\right)$ are in close contact with the isothermal wall boundary condition. For this reason even a positive streaming velocity does not increase the mean temperature. Just outside the boundary 
layers $\left(r \approx R-\pi \delta_{\kappa}\right)$, convection is the main heat transfer mechanism. Due to the negative streaming velocity at this radial coordinate, colder fluid is convected to the left and the mean temperature is reduced. For even smaller radial coordinates the streaming velocity is smaller and for this reason the mean temperature profile it not affected.

In the case of the isothermal no-slip boundary condition, the mean temperature profile in the center of the tube is nearly linear. This is due to the absence of small vortices, as can be seen in the streaming flow field shown in Figure 5.5 a). Only one large vortex occurs, which extends over the whole inner part and leads to a homogeneous flow field. On the other hand, the slightly wobbly mean temperature profile in the case of the isothermal slip wall boundary condition can be explained with the irregular streaming velocity field shown in Figure $5.5 \mathrm{c}$ ). The small vortices convect heat and create a nonhomogeneous flow field. The inhomogeneous flow field influences the mean temperature profile, which leads to the wobbly temperature profile. The large vortex over the right boundary condition shown in Figure $5.5 \mathrm{c}$ ) also has a large influence on the mean temperature profile. As can be seen in Figure 5.8, the temperature stays nearly constant for large values of $x$, as the vortex convects fluid into the domain with a constant temperature of $T_{H}=325 \mathrm{~K}$.

In Figure 5.11 the averaged mean temperature is compared with the mean temperature profile from the one-dimensional model given in Chapter 5.1.1.2. Only the results for the isothermal no-slip and adiabatic slip boundary conditions are presented, as the heat transfer coefficient in the one-dimensional model is not defined in the other boundary conditions and because the two others CFD simulations show similar results. The solid lines show the CFD results, while the dotted lines show the results obtained from the onedimensional model. In both wall boundary condition cases the difference between the one-dimensional model and the CFD simulation is small. Still, deviation due to the twodimensional effects like streaming can be seen. In the case of an isothermal no-slip boundary condition the profile is shifted towards smaller $x$-values in the simulation, while the one-dimensional model overlays the linear profile between the heat exchangers. In the isothermal slip case (not shown) this effect is even more pronounced due to the larger streaming. Another difference between the CFD simulation and the on-dimensional model is the change in the mean temperature at the extremities of the thermal buffer tube, which are slightly higher in the CFD simulation than in the one-dimensional model.

The differences between the one-dimensional model and the CFD simulations are smaller in the case of the adiabatic slip boundary condition as the streaming is two orders of magnitude smaller than in the other isothermal no-slip case.

It was shown that streaming has an important effect on the mean temperature profiles inside of a thermal buffer tube. Deviations from the one-dimensional model increase with increasing streaming. If the two-dimensional streaming effects shall be taken into account, CFD is an appropriated tool, as was shown by comparing with the different simplified models. 


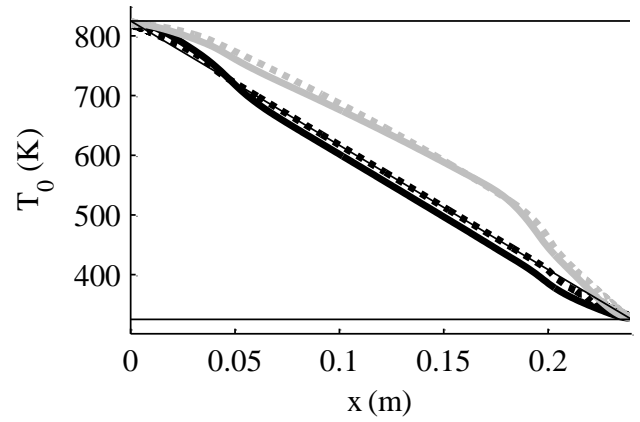

Figure 5.11: Cross sectional and time averaged temperature from CFD (solid lines) and one-dimensional (dotted lines) models over the length $x$ of the thermal buffer tube for two different wall boundary conditions: isothermal no-slip boundary condition in black and adiabatic slip boundary in gray. The thin black lines indicate the temperature of the heat exchangers and the linear profile between them.

The influence on the acoustic field due to the changes in temperature can be seen when looking at the reflection coefficient. Figure 5.12 shows the reflection coefficient over the length $x$ of the thermal buffer tube for the four different wall boundary conditions. The thin black line shows the reflection coefficient if a linear temperature profile and no reflection at the right side are assumed. The deviations of the CFD results from this line are partially due to the different mean temperature profiles, as the local temperature is determining the local mean density and the local speed of sound and thus the characteristic impedance. Another reason for differences is the non-reflecting boundary condition which introduces a small amount of reflection at the right of the domain.
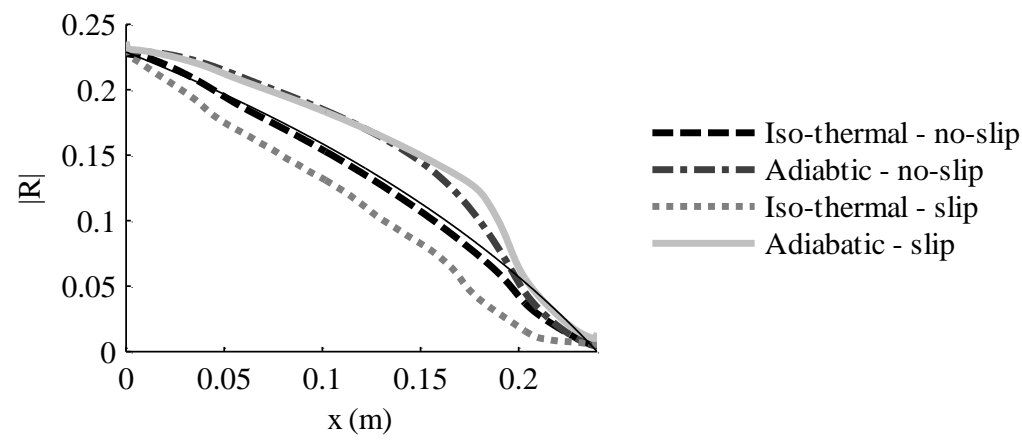

Figure 5.12: Absolute value of the reflection coefficient over the axial coordinate $x$ for four different wall boundary conditions. Thin black line shows the theoretical reflection coefficient if a linear temperature profile is assumed and no reflections occur at the right side of the domain. 
The reflections at the left and right of the domain can be compared with the theoretically expected ones. The reflection coefficients are summarized in the Table 5.2. At the right side of the domain it is assumed that no reflections occur. In the CFD simulations this is realized with the non-reflecting boundary conditions. The correct implementation and performance of this boundary condition can be verified by looking at the reflection coefficients at the right side of the domain. In the CFD simulations these reflections are below $1 \%$, which is assumed to be sufficiently low as this is at least one order of magnitude smaller than the reflections that are induced inside the domain due to the temperature changes. This small amount of reflection is due to numerical errors in the boundary treatment.

The reflection coefficient at the left side is the result of the changes in the characteristic impedance $Z_{0}=\rho_{0} c_{0}$ inside of the domain. The theoretical reflection coefficient can be calculated from the characteristic impedance at the hot and cold side as follows [50]:

$$
R=\frac{Z_{0, C}-Z_{0, H}}{Z_{0, C}+Z_{0, H}}
$$

For all cases, the values from the simulation match the theoretical values within $1.5 \%$, even though the profiles within the domain are different due to the different temperature profiles.

Table 5.2: Reflection coefficients at both extremities of the thermal buffer tube for the four CFD simulations and analytical values.

\begin{tabular}{ccc}
\hline & $\boldsymbol{R}(\boldsymbol{x}=\mathbf{0})$ & $\boldsymbol{R}(\boldsymbol{x}=\boldsymbol{L})$ \\
\hline Isothermal no-slip & 0.227 & 0.004 \\
Adiabatic no-slip & 0.230 & 0.004 \\
Isothermal slip & 0.226 & 0.005 \\
Adiabatic slip & 0.231 & 0.010 \\
Theoretical & 0.228 & 0 \\
\hline
\end{tabular}

\subsubsection{Energy losses}

The main goal of the thermal buffer tube is to thermally insulate the hot heat exchanger from the ambient heat exchanger, while efficiently transmitting the acoustic power out of the hot zone [14]. Due to the wall temperature gradient, acoustic power is dissipated inside the thermal buffer tube. The dissipated acoustic power for the different wall boundary conditions is shown in Table 5.3. The first column gives the acoustic power calculated from the time integral [6]: 


$$
\dot{E}_{a c}=\frac{\omega}{2 \pi} \oint p(t, x) U(t, x) d t
$$

and the second column gives the acoustic power calculated from complex amplitudes [6]:

$$
\dot{E}_{a c}=\frac{1}{2} \operatorname{Re}\left(p_{1} U_{1}^{*}\right)
$$

The acoustic power dissipated is small compared to the acoustic power transmitted: $E_{a c} \approx 1500 \mathrm{~W}$. The highest dissipation occurs in the case of the isothermal slip wall boundary conditions. The dissipation in this case is even higher than the dissipation from the isothermal no-slip wall boundary condition. This is due to the temperature gradient at the wall, which induces the highest losses. In the case of the no-slip wall, the viscous boundary layer thermally insulates the wall from the fluid inside of the thermal buffer tube, reducing the losses.

Table 5.3: Acoustic power dissipation for four different wall boundary conditions and calculated from CFD data using two different methods (first two columns). The third column gives the theoretic acoustic losses for a cylindrical tube with a constant wall temperature of $T=575 \mathrm{~K}$. The acoustic power at the inlet is $\dot{E}_{a c, i n}=1500 \mathrm{~W}$.

\begin{tabular}{cccc}
\hline $\begin{array}{c}\text { Wall boundary } \\
\text { condition }\end{array}$ & Time integral & $\begin{array}{c}\dot{\boldsymbol{E}}_{\boldsymbol{a c , \text { in }}}-\dot{\boldsymbol{E}}_{\text {ac,out }}(\mathrm{W}) \\
\text { First order } \\
\text { amplitude }\end{array}$ & $\begin{array}{c}\text { Cylindrical } \\
\text { tube }\end{array}$ \\
\hline Isothermal no-slip & 5.7 & 5.8 & 2.8 \\
Adiabatic no-slip & 0.4 & 0.6 & 1.2 \\
Isothermal slip & 10.0 & 10.3 & 1.6 \\
Adiabatic slip & 0.03 & 1.0 & 0 \\
\hline
\end{tabular}

Comparing the values of the acoustic power dissipation obtained from the two different methods, one can see that the time integral method leads to smaller acoustic power dissipation than the method in which the first order amplitude from the Discrete Fourier Transform is used. The time integral method incorporates also higher harmonics of the pressure and the volume flow rate. The difference between the two methods thus shows the influence of the higher harmonics on the acoustic power dissipation. This effect is especially pronounced in the case of the adiabatic slip wall boundary condition, which is also the case in which the largest second order pressure amplitudes occur. The second order pressure amplitudes are at least twice as high for the other cases with different wall boundary conditions. The higher orders in the volume flow rate are similar for all wall boundary conditions. This means that in the case of the adiabatic slip wall boundary condition, acoustic energy is transferred to higher order pressure amplitudes. In a real engine 
only the first order quantities take part in the thermoacoustic effect, for this reason the higher order effects are considered to be losses. The acoustic power dissipation calculated with the first order amplitudes is thus most appropriate for comparison.

The acoustic power losses in the thermal buffer tube can be compared with the theoretic acoustic power losses in a cylindrical tube. The acoustic losses in a cylindrical tube are calculated using the propagation constant $\Gamma$ given in Equation (2.71) and assuming a pure traveling wave. The required thermoacoustic functions are calculated using a boundary layer approximation (see Appendix B) for a mean temperature of $T=575 \mathrm{~K}$, which corresponds to the center of the thermal buffer tube. The dissipated acoustic power is calculated as follows:

$$
\dot{E}_{a c, \text { out }}-\dot{E}_{a c, \text { in }}=\frac{1}{2}\left|p_{1}\right|\left|U_{1}\right|(1-\exp (-2 L \cdot \operatorname{Re}(\mathrm{k})))
$$

The results are given in the third column of Table 5.3 for the different boundary conditions. The acoustic losses inside a cylindrical tube with a constant temperature and the thermal buffer tube are on the same order of magnitude. Apart from the case with adiabatic no-slip wall boundary conditions the acoustic losses from the cylindrical tube are smaller than those found in the thermal buffer tube simulations. The differences between both values characterize mainly the effect of the temperature gradient at the wall. The difference is especially pronounced for the case with isothermal slip wall boundary conditions. The reason why the acoustic losses are lower in the thermal buffer tube for the adiabatic no-slip wall is not clear and needs further investigation.

The next loss mechanism investigated in this chapter is the heat that is transferred over the wall. Figure 5.13 shows the time averaged heat flux from the wall into the fluid over the length of the thermal buffer tube for the two cases with an isothermal wall boundary condition. In the case of a no-slip boundary condition the heat transfer occurs close to the extremities: inside of the thermal buffer tube the heat flux over the wall is nearly zero. The high values of heat transfer at the extremities of the thermal buffer tube are due to the interaction of the ideal heat exchanger with the acoustic flow and the wall. This can be shown with Figure 5.14, where the mean temperature is shown close to the left heat exchanger. Each line represents the mean temperature at locations separated by $\Delta x=3.2 \mathrm{~mm}$. Whether heat is transferred from the wall to the fluid or from the fluid to the wall can be seen by looking at the temperature gradient at the wall. This gradient is defined by the effects happening inside the boundary layer. For this reason the heat transfer looks different than one would expect when looking at the surface and the time averaged temperature profile in the thermal buffer tube shown in Figure 5.8. Boundary layers induced by an acoustic wave experience a phase delay. The interaction of this phase delay with the ideal heat exchanger boundary conditions leads to the mean temperature profiles shown in Figure 5.14 and finally to the heat fluxes shown in Figure 5.13. In a real 
engine this effect would be smaller as the wall temperature is not constant there and the heat exchanger is not ideal.

In the case of the isothermal slip wall boundary condition, the heat flux fluctuates more at the inside of the domain compared to the isothermal no-slip boundary case. This is due to the smaller scale streaming patterns shown in Figure $5.5 \mathrm{c}$ ) and the non-existing viscous boundary layer, which would otherwise thermally insulate the wall from the bulk of the thermal buffer tube. Furthermore, one can see that the heat flux values inside of the thermal buffer tube are larger than zero. This has to do with the surface and time averaged temperature profile which is lower in most of the thermal buffer than the wall temperature. Still the same effects as for the isothermal no-slip wall boundary condition case occur at the extremities.

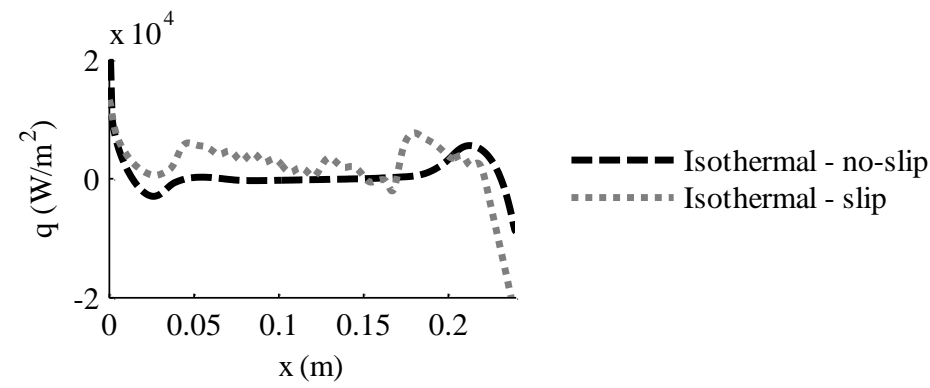

Figure 5.13: Time averaged heat flux from the wall to the fluid over the length of the thermal buffer tube and for the two cases with isothermal wall boundary conditions.

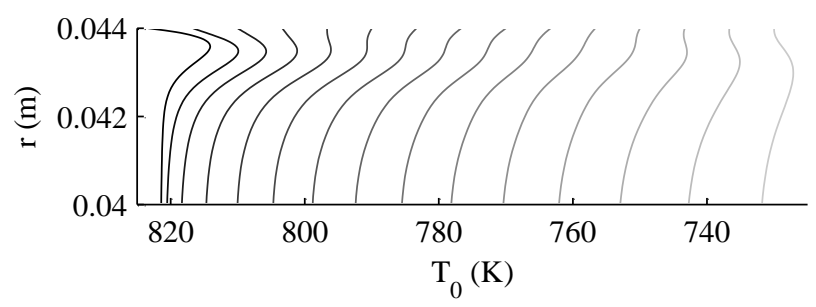

Figure 5.14: $\quad$ Mean temperature at different $x$-locations over the radius. Each line shows the temperature of the fluid over the radius at different axial positions starting from the hot heat exchanger and separated by $\Delta x=3.2 \mathrm{~mm}$ each. Lighter gray lines indicates higher $x$-cooordinate.

By integrating the heat flux over the total thermal buffer tube wall, the total amount of heat transferred can be obtained. The results are shown in Table 5.4 for the two isothermal wall boundary conditions. The first column gives the integral of the heat flux from the wall to the fluid,

$$
\dot{Q}=\int q d A
$$


while the second one gives the integral of the absolute value of the heat flux.

$$
\dot{Q}_{a b s}=\int|q| d A
$$

As was mentioned earlier, the heat transferred is higher for the slip wall boundary condition as no viscous boundary layer insulates the wall from the bulk of the thermal buffer tube. The values given in the table can be compared with the heat that is conducted through the wall of the thermal buffer tube. Assuming a stainless steel wall with thickness of $h=4 \mathrm{~mm}$ and heat conductivity of $k=18.7 \mathrm{~W} /(\mathrm{m} \mathrm{K})$ [7] the heat conduction through the thermal buffer tube wall due to the temperature gradient can be estimated:

$$
\dot{Q}=-k A_{\text {wall }} \frac{d T}{d x}=45 \mathrm{~W}
$$

This is on the same order as the heat that is added to the wall due to the acoustic wave or even smaller. The assumed thermal wall boundary condition is thus neither ideally adiabatic nor ideally isothermal, but in between. For this reason it would be interesting in a future study to integrate the thermal buffer tube wall into the simulations. But this is a demanding simulation as it would involve that the fluid structure interaction is resolved and that the simulated time is extended. This is a consequence of the high heat capacity of the stainless steel wall compared to helium and hence slower temperatures changes in the wall.

Table 5.4: The heat transferred $\dot{Q}$ from the wall to the fluid for the two isothermal wall boundary conditions is given in the first column, while in the second column the integral of the absolute value of the heat flux $\dot{Q}_{a b s}$ over the thermal buffer tube wall is given.

\begin{tabular}{ccc}
\hline Wall boundary condition & $\dot{\boldsymbol{Q}}(\mathbf{W})$ & $\dot{\boldsymbol{Q}}_{\boldsymbol{a b s}}(\mathbf{W})$ \\
\hline no-slip & 39.1 & 96.7 \\
slip & 133.6 & 262.0 \\
\hline
\end{tabular}

Furthermore, it has to be noted that the heat transferred over the wall is higher than the acoustic power dissipated. This may originate from either the transient effect due to the initialization or from the ideal heat exchanger boundary conditions at both ends of the domain. For this reason, the heat that is transferred from the ideal heat exchanger boundary condition into the thermal buffer tube will have to be investigated in more detail. The energy convected inside the domain can be calculated as the time averaged inner energy convected over the boundaries as follows:

$$
\dot{E}_{\text {inner, }, \text { in }}=\frac{\omega}{2 \pi} \iint_{t A} u \rho c_{v} T d A d t
$$


The values obtained from these calculations are subject to large errors because the time averaged values are already three orders of magnitude smaller than the maximum time dependent value. The difference between the inlet and the outlet is again one order of magnitude smaller than the average value. Furthermore, the mean density and the mean temperature are large compared to their oscillating part. For these reasons the values of the energy convected over the boundary are subject to some doubts. These doubts are augmented as no clear mesh independency could be reached in this derived quantity. This is especially true in the case of slip boundary conditions in which a clear mesh dependency can be seen. For this reason no values are presented. Additional work has to be done to achieve more reliable results in the calculation of the inner energy convected over the boundary. This future work would allow closing the energy balance over the whole thermal buffer tube.

\subsubsection{Conclusion and recommendations}

In this chapter, the flow field inside a thermal buffer tube is revealed and the influence of two-dimensional streaming patterns on the temperature field is shown. The streaming velocity and the mean temperature profiles are validated with reduced models. The acoustic energy losses and the heat transfer over the wall is characterized, but in order to evaluate the heat convected over the boundaries, further work is needed.

Moreover, the simulations illustrate the scale problem of CFD simulations in thermoacoustics, as the initial temperature profile has a large influence on the temperature profile after 300 periods. This is due to the different time scales between the acoustics and the heat conduction. For this reason future simulation should be initialized with a temperature profile that is closer to the profile that will be reached if the simulations are done for a long enough time that the conductive effects also reach a steady periodic case. This is done by using the one-dimensional model with higher heat conduction in the axial direction and reducing slowly the heat conductivity during the calculation to the physical value. Once the steady periodic state is reached, the temperature of the reduced simulation at the time instance when no velocity occurs can be applied to initialize the simulation.

Furthermore, additional work has to be done on the boundary conditions, such that a non-reflecting inlet can be used. A boundary condition that promises better results is the time domain impedance boundary condition that was implemented by van der Poel into ANSYS CFX [41] within the scope of his maser thesis [97] following the work of Huber et al. [98]. An implementation into ANSYS Fluent is difficult, as this boundary condition uses the gradients at the boundary and these are not directly available in ANSYS Fluent. As the characteristic boundary condition uses the gradients at the boundaries, it is expected to be less influenced by the temperature gradient inside the thermal buffer tube. This means that with this boundary condition it would probably be possible to implement a non-reflecting boundary condition at the hot heat exchanger that does not introduce a 
drift in pressure or velocity. This would allow conducting simulations with a wave phasing that is closer to the phasing that occurs in real engines and the investigation of the influence of different phasing on the streaming patterns. The boundary conditions can also be extended such that they introduce different wave shapes in order to reveal the influence of higher orders on the streaming patters.

In a further step, the CFD model can be extended to incorporate additional effects that were not yet considered. One interesting additional effect is gravity, as it can have both positive and negative effects on streaming [96, 99]. Another effect that is not taken into account in this work is the heat transfer inside the wall, which could lead to reduced heat losses as the wall temperature would get in equilibrium with the bulk flow. In the simulations radiation is neglected even though that due to the high temperature of the heat exchanger it has an effect on the heat distribution in the thermal buffer tube and the heat losses [9]. At higher amplitude turbulence becomes important and a reliable turbulence model is needed. This is especially challenging as transition and relaminarization effects due to the oscillatory flow should be taken into account. But it is also of special interest as the overall effect of turbulence on the heat leaks in thermal buffer tubes is unclear [96]. Experimental investigation is preferred over CFD, as no appropriate turbulence models for the purely oscillating flow are available in commercial CFD software packages at the moment and direct numerical simulations are very time consuming. Even though more steps have to be done in order to implement the complete physics of the thermal buffer tube, a first step was done in revealing the streaming patterns and the consequences on the mean temperature. 


\subsection{Acoustic flow in a U-bend 1}

In a traveling wave device the necessary wave phasing is achieved by feeding back the acoustic wave. Several types of feedback exist; the first and most common one uses pipes of different cross-section to loop the acoustic wave. The different cross-sections lead to a well-defined inertance and compliance, such that the desired traveling wave phasing in the resonator is obtained [14]. Another possibility that has been investigated in recent years uses an electrical feedback [100, 101]. In these engines the thermoacoustic core, consisting of the heat exchangers and the regenerator, are comprised between two transducers. The transducers are electrically modulated such that traveling wave phasing is achieved in the regenerator, leading to an amplification of the acoustic wave in the device. Looping back electrically has the big disadvantage that an additional transducer is needed, which increases the number of moving parts and increases the complexity.

For this reason the acoustical feedback loop with pipes of different cross-section is investigated further in this chapter. The present work is done in the scope of reducing the size of thermoacoustic devices, which leads to increasingly sharp bends. In the most extreme case of a coaxial device the flow is redirected by a flow redirection plate where additional losses and flow effects can be expected. Interaction with the nearby heat exchangers is presumed to add additional losses [102].

The most important papers on acoustic flow in a bend were published from the 1970's on. Numerous solutions of the flow in a bend have been derived since then and Rostafinski [103] reviewed these solutions that solve the linearized wave equations by three different methods: by separation of the variables, with the perturbation principle or numerically.

Most of the reviewed works solve the linearized equations in the frequency domain and do not resolve vortex generation that can occur for sharp bends and high displacement amplitudes. Only few works in which vortex generation in a bend is resolved can be found, one is the work of Dequand et al. [104, 105]. They solve the non-linear Euler equations for inviscid and compressible flows, to estimate the acoustic response of a $90^{\circ}$ bend. They differentiated between situations with and without mean flow and observed different results depending on the geometry of the bend. In their work, Zink et al. [44] used CFD to reveal the influence of a $90^{\circ}$ bent resonator on the performance of a standing wave engine. They showed that bending the resonator reduces the efficiency of the engine and that the curvature influences the resonance frequency. Lycklama à Nijeholt [102] investigated qualitatively a compact coaxial traveling wave engine, which has a

${ }^{1}$ The work presented in this chapter has been carried out together with Rob Boumans in the scope of his master thesis: R. Boumans; Quantification of acoustic losses in a bended waveguide using CFD; University of Twente; 2014 
flow redirection plate at the extremity. They show that a strong vortex is created that affects the temperature distribution in the engine and which is assumed to affect the efficiency of the engine. But in their study the effect of the vortex is not quantified in more detail.

Next to the numerical and analytical work, experiments with U-bends and coils have been conducted using liquids as working fluid. Iguchi et al. [106] investigate a U-bend with negligible curvature and reach a good agreement with their model for energy dissipation. Furthermore, they predict the transition from laminar to turbulent in the U-bend. Olson and Swift [107] could extend the experimental parameter range for higher amplitudes and high shear wave numbers $s$ with their experiments and separated the dissipation in the straight section from the one in the bent section.

In this work the flow field in the bend is investigated with CFD simulations which solve the full Navier Stokes equations numerically, including the viscous effects. This allows resolving the vortex generation not included in the analytical solutions and the boundary layer effects not resolved in the work of Dequand et al. [104, 105].

A first step to reveal the acoustic flow in a sharp bend for application in thermoacoustic devices with a flow redirection plate is done, by investigating a U-bend. The study is limited to geometric effects on the flow field, which means that the frequency and the pressure amplitude of the wave are kept constant at $f=100 \mathrm{~Hz}$ and $p_{1}=100 \mathrm{~Pa}$ respectively, while the bend radius and the cross-sectional area are varied. The frequency corresponds to a low reduced frequency and is on the order of what is used in thermoacoustic devices [108]. The pressure amplitude is low for thermoacoustic applications, but allows for a first estimation of the geometric effects in a bend.

In the design of a thermoacoustic device, the acoustic characteristics of sharp bends have to be taken into account in order to reach high efficiencies. For this reason the influence of the bend geometry on the reflection coefficient and the deviations from the low reduced frequency model are investigated. Components that are in the vicinity of the bend can be influenced by the distortion of the flow field due to the transition from straight to bent. Therefore, the transition length from bent to straight is determined in this chapter. Finally, the flow field for extremely sharp bends, in which vortices occur, is revealed and the limit for vortex generation is given.

\subsubsection{Bend models}

In order to reduce the required computational costs, the bend is reduced to a twodimensional curved layer in a first approach. This geometric reduction still allows for a first investigation of the geometric effects, like the curvature and the half spacing, on the flow in a U-bend, but the computational costs are reduced considerably. The corresponding CFD model is presented in Chapter 5.2.1.1 and in Chapter 5.2.1.2 the low reduced frequency model to which the simulation results are compared is summarized. 


\subsubsection{Curved layer CFD model}

In this chapter the two-dimensional CFD model of the bent waveguide is presented. The model consists of a U-bend. A general representation of the geometry can be found in Figure 5.15. The wave enters the domain through the non-reflecting boundary condition at the inlet, propagates through the first straight section, then the bend and the second straight section. Finally, the wave leaves through the outlet, where a non-reflecting boundary condition is imposed. The straight sections are one tenth of the wavelength long, which is large enough such that the wave propagates as a plane wave close to the non-reflecting boundary condition. This allows estimating the wave propagation constant needed for the non-reflecting boundary condition from the analytical solution for parallel plates given in Appendix B. In order to show the influence of the geometry, the radius of the bend $R$ and the half spacing $h$ are varied in this study.

The working fluid is helium and is modeled as an ideal gas with the fluid properties as defined in Appendix E. The acoustic wave has a frequency of $f=100 \mathrm{~Hz}$ and a pressure amplitude of $p_{1}=100 \mathrm{~Pa}$. The walls are modeled as isothermal and no-slip. The flow regime inside the bent waveguide can be estimated according to Olson and Swift from the Reynolds number and the ratio of the radius to the viscous penetration depth [84]. The maximum values are calculated using the maximum half spacing $h_{\max }$ used in this study:

$$
\begin{gathered}
R e=\frac{2\left\langle u_{1}\right\rangle h_{\max } \rho_{0}}{\mu_{0}}=256 \\
\frac{h_{\max }}{\delta_{v}}=42
\end{gathered}
$$

According to Olson and Swift [84] the flow field is laminar for all present simulations. This means that no turbulence model is needed.

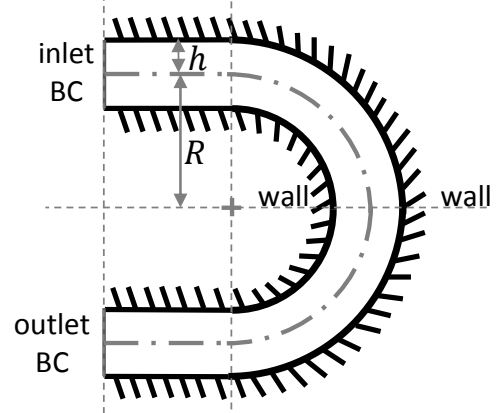

Figure 5.15: Schematic overview of computational domain.

The mesh used in the CFD simulations has a similar structure to the mesh used in the straight pipe validation shown in Chapter 4.4. The domain has a hexahedral mesh as this allows decoupling the wave propagation direction from the cross-direction. The discreti- 
zation of the domain is given schematically in Figure 5.16. In the wave propagation direction the cells have a constant length in the straight part and a uniform distribution in the $\varphi$-direction in the bent part. In the cross-direction the elements grow geometrically with a maximum geometric growing factor of 1.2 from the walls towards the center line. The element size and the growth factor are chosen such that there are at least 10 elements inside the viscous boundary layer and that the maximum elements size is less than $20 \%$ of the half spacing $h$.

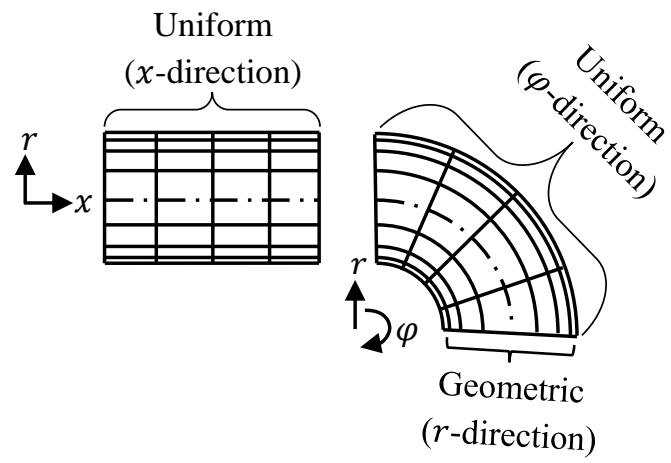

Figure 5.16: Schematic of the computational mesh distribution.

As a consequence of the above defined mesh, a jump in element size occurs close to the walls at the transition from the straight to the bent part. This jump in size can result in extra reflections in the domain as a result of the small flow field discontinuities between the two cells. The magnitude of these reflections depends on the dimensionless curvature and the shear wave number, but is negligible compared to the reflection generated by the geometry in all simulated cases.

In total five periods are simulated, which corresponds to a total simulated time of $t=0.05 \mathrm{~s}$, and allows the initial transient behavior to damp out. The time is discretized with a least 160 time steps per period.

\subsubsection{Low reduced frequency model}

The results obtained with the CFD model are compared to the low reduced frequency solution. As no analytical solution for the entire domain exists, the simulation results are compared to the low reduced frequency solution introduced in Chapter 2.3 for parallel plates and curved layers in the respective part. The results for the curved layer are summarized in the following:

$$
p_{1}=A \exp \left(\Gamma \frac{R \omega}{c_{0}} \varphi\right)+B \exp \left(-\Gamma \frac{R \omega}{c_{0}} \varphi\right)
$$




$$
\begin{gathered}
u_{1}=\frac{i}{\omega \rho_{0}}\left(1-h_{v}\right) \frac{1}{r} \frac{\partial p_{1}}{\partial \varphi} \\
T_{1}=\frac{1}{c_{p} \rho_{0}}\left(1-h_{\kappa}\right) p_{1}
\end{gathered}
$$

The results for parallel plates have a similar structure and can be found in Chapter 2.3. In order to compare the analytical solution with the CFD results, the simulation results have to be taken far enough from the junction between the straight and curved part. The exact distance needed will be discussed in more detail in the results section.

Furthermore, the low reduced frequency solution allows the determination of the two dimensionless parameters of interest that define the acoustic flow in the bend: the viscous shear wave number

$$
s=h \sqrt{\frac{\rho_{0} \omega}{\mu}}
$$

and the dimensionless bend radius

$$
\chi=\frac{R}{h}
$$

In the simulation the shear wave number is varied by changing the height of the domain, as the other parameters are kept constant throughout this chapter. The dimensionless radius $\chi$ is then adapted by varying the radius $R$ accordingly. In Figure 5.17 some typical geometries with the corresponding dimensionless numbers are given to visualize the bends investigated in this study.

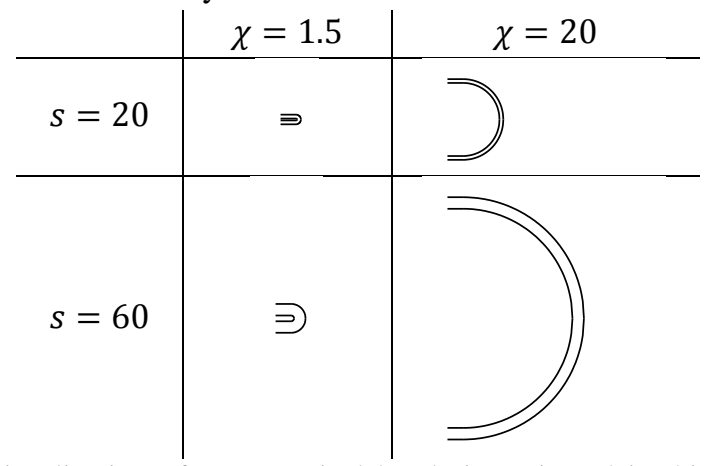

Figure 5.17: Visualization of some typical bends investigated in this study with their corresponding dimensionless numbers.

\subsubsection{Results and discussion}

In this chapter the results from the CFD simulations of the acoustic flow in a curved layer are presented. First, the results are compared to the respective low reduced frequency model in a curved layer discussed in Chapter 2.3.4. The comparison is done for a case 
with a high dimensionless radius of $\chi=10$, where the low reduced frequency approximation is assumed to be valid. Only the results in terms of pressure and velocity amplitudes are compared, as the density and temperature field behave in a similar way.

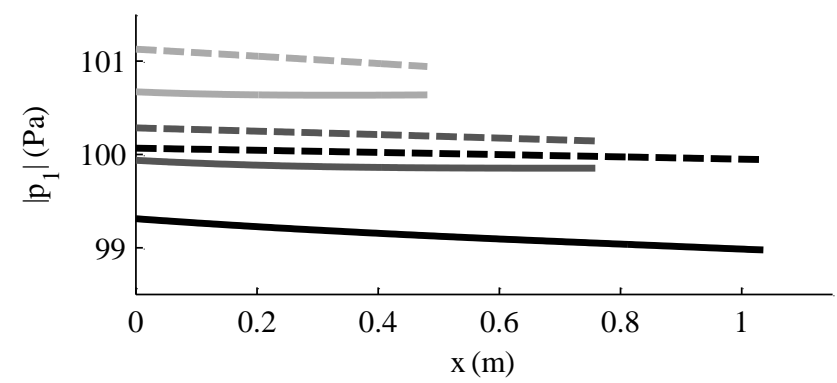

Figure 5.18: The pressure amplitude $p_{1}$ over the axial coordinate for constant dimensionless bend radius $\chi=10$. The black, dark gray and light gray lines correspond to a shear wave number of $s=60,40$ and 20, respectively. Results from simulations are shown with solid lines, while analytical results are shown with dashed lines.

In Figure 5.18 the pressure amplitude $p_{1}$ is plotted over the axial coordinate for three different shear wave numbers $s$ of 60, 40 and 20. The simulation results are compared to the analytical solution from the low reduced frequency approximation and differ by less than $1 \%$. All pressure amplitudes obtained from simulations have a lower value than in the corresponding analytical solution, indicating that additional damping occurs in the CFD simulations. Additionally, a mean pressure drift occurs in the domain which in the worst case reaches a maximum of $2 \mathrm{~Pa}$ within the first five periods. The differences between the analytical solution and the CFD simulations have the same order of accuracy as the validation case in Chapter 4. Thus, the bend does not introduce additional inaccuracies.

The velocity amplitude decay is similar to the pressure amplitude decay. Hence, only the axial velocity profile over the height is shown here. The velocity profile half way through the bend is shown as solid lines in Figure 5.19 and compared to the analytical solutions shown as dashed lines. The axial velocity is scaled according to Equation (5.27) as follows:

$$
u_{1} \frac{\omega \rho_{0}}{i} R\left(\frac{d p_{1}}{d \varphi}\right)^{-1}=\left(1-h_{v}\right) \frac{R}{r}
$$

where $h_{v}$ is the shape function as defined in Chapter 2.3. The lines from the simulations and the analytical solution perfectly overlay each other indicating that the CFD simulations correctly predict the flow field in a curved layer. 


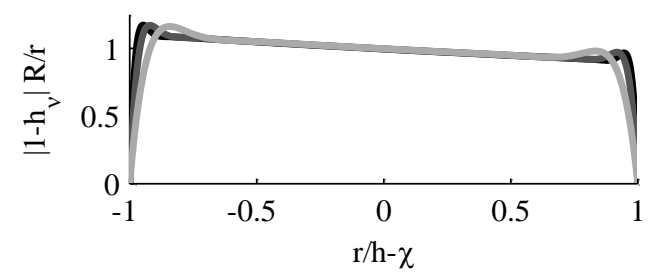

Figure 5.19: Scaled velocity profile of the axial velocity in the middle of the bend over the dimensionless height, for a dimensionless radius of $\chi=10$ and three shear wave numbers. The light gray, dark gray and black lines correspond to a shear wave number of $s=20,40$ and 60 respectively. The solid and the dashed lines represent the results from the simulations and the low reduced frequency model respectively.

In the following, the flow field in a curved layer will be investigated in more detail. In Chapter 5.2.2.1 the reflection induced by the curvature is discussed and in Chapter 5.2.2.2 the transition effects from the straight to the curved pipe are revealed. In Chapter 5.2.2.3 the geometry is changed in such a way that the limits of the analytical solution are reached, and finally in Chapter 5.2.2.4 the flow field for extreme curvatures is given.

\subsubsection{Curvature induced reflections}

The reflections induced by the bend have consequences for the design of the acoustic motor as the phasing of the velocity and pressure is altered in the regenerator and thus has to be taken into account by adapting the inertance and compliance of the thermoacoustic engine.

In this chapter the influence of the geometric parameters on the reflections induced by a U-bend are investigated. The reflections due to the bend $R_{\text {bend }}$ can be quantified when subtracting the reflections at the end of the bend from those in the beginning of the bend. The values for constant shear wave number $s=60$ and different curvatures are shown in Table 5.5. The values for dimensionless bend curvatures $\chi>5$ have negative values. The reason for this is the low value of the reflection coefficient in these cases. Reflections mainly occur at the outlet of the domain and due to dissipation, the amplitudes of the forward $f$ and backwards traveling wave $g$ decrease in their respective wave propagation direction. This means that the reflection coefficient, which is the ratio of the backwards traveling wave to the forward traveling wave, increases with the axial coordinate, which leads to the negative reflection coefficient.

For bend sharpness $\chi<10$ the reflections due to the bend are positive. The latter indicates that the backward traveling waves increases in its wave propagation direction. This is only possible if the bend introduces reflections that lead to this increase. An explanation for the increase in reflections in the bent part of the domain is not trivial. The speed of sound is not constant over the cross-section of the bend, as can be illustrated by looking at the distance the wave has traveled on the inside and outside of the bend. The 
distance on the inside of the bend is much shorter than that on the outside, while the wave still propagates as a plane wave with regard to the bend angle $\varphi$. These radial changes in propagation speed can lead to local reflections in the bend. For sharper bends, the changes in propagation speed in radial direction are larger, thus resulting in higher reflection coefficients.

Table 5.5: $\quad$ Reflections caused by the bend $R_{\text {bend }}$ as a function of the dimensionless bend curvature $\chi$.

\begin{tabular}{cc}
\hline$\chi$ & Reflection coefficient \\
\hline 20 & $-1.4 \cdot 10^{-3}$ \\
10 & $-2.0 \cdot 10^{-4}$ \\
5 & $1.0 \cdot 10^{-3}$ \\
3 & $2.7 \cdot 10^{-3}$ \\
1.5 & $4.7 \cdot 10^{-3}$ \\
\hline
\end{tabular}

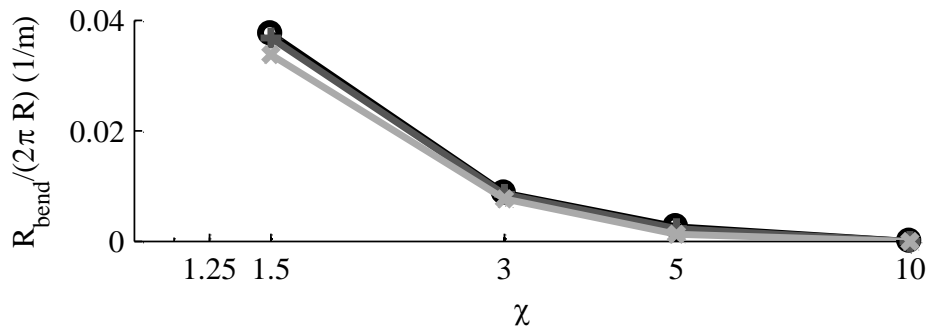

Figure 5.20: Reflections per length caused by the bend as a function of the dimensionless bend radius for shear wave numbers $s$ of 20, 40 and 60, in light gray, dark gray and black respectively. The total reflection is corrected for the reflections caused by the non-reflecting boundary condition, such that only the reflection of the bend is shown and is then divided by the length over which it has propagated.

Figure 5.20 shows the reflections per length caused by the bend for other shear wave numbers. The reflections are calculated as the difference of the reflection coefficient at the begin and end of the bend, divided by the bend length in the wave propagation direction, in order to get comparable results. From Figure 5.20 it can be concluded that the shear wave number in the range investigated has no influence on the magnitude of the reflection per length, while the dimensionless bend radius has a large influence on the reflection per length introduced by the bend. For smaller dimensionless radii and thus sharper bends, the reflection increases significantly. 
The influence of the extra reflections on the approximation of the acoustic losses in the simulated flow fields is very small. Even with a reflection coefficient of $10 \%$ the error in the acoustic power would be smaller than $2 \%$. But the reflections induced by the bend do have consequences for the design of the acoustic motor as the phasing of the velocity and pressure is altered in the regenerator and thus has to be taken into account by adapting the inertance and compliance of the thermoacoustic engine.

Summarizing the above presented results, it can be concluded that sharp bends introduce extra reflections and that the shear wave number in the investigated range has no influence on the reflections.

\subsubsection{Transitions from straight to bent}

Between the bend and the straight part a transition has to occur as the velocity profiles are different in both parts. This can be seen from the different axial velocity profiles obtained from the low reduced frequency approximations of the parallel plate and the curved layer given in Chapter 2.3. This means that components nearby such as heat exchangers can be influenced by the bend. Because these effects are not incorporated in the low reduced frequency approximation, they are investigated with CFD in this work.

In Chapter 2.3 it is shown that the axial velocity amplitude $u_{1}$ in the bend is a function of the cross-directional coordinate, while in the straight part the axial velocity outside the boundary layer is not a function of the cross-directional coordinate. For the design of the thermoacoustic engine it is important to know how far the asymmetry extends into the straight domain and how strong this effect is for different geometries. The transition between the two profiles is investigated by using the cross-directional derivative of the axial velocity $\partial\left|u_{1}\right| / \partial r$ on the center line, as it is zero in the straight part and has a negative value in a bend.

Figure 5.21 shows the normalized radial derivative of the axial velocity for three different shear wave numbers $s=20,40$ and 60 and three different dimensionless radii $\chi=10,5$ and 3 over the axial coordinate normalized with the half spacing $h$. The crossdirectional derivative of the axial velocity $\partial u_{1} / \partial r$ on the center line is scaled with the bend radius $R$ divided by the velocity amplitude $\left|u_{1}\right|$ on the center line. $x=0$ indicates the transition point between the straight part, $x<0$ and the bend part $x>0$.

As all the simulated cases overlay each other in the transition region from straight to bend, the length of the transition area scales with the half spacing $h$ and is not a function of the curvature. The transition is thus driven by the viscous dissipation in the boundary layer and not by the displacement amplitude. As the profile is symmetric around the transition point from straight to bent, the transition effects are the same in both the bent and straight part.

In the bent part of the geometry the magnitude of the scaled velocity derivative stays higher than minus one for some cases. An explanation for this can be found in the lengths of the transition area. For increasing bend sharpness, the total length of the bend becomes 
smaller, while the transition length remains the same for equal shear wave numbers. The consequence is that the transition regions in sharp, $180^{\circ}$ bends overlap and that the velocity profile does not have the length to complete the transition from the straight profile to the bent profile.

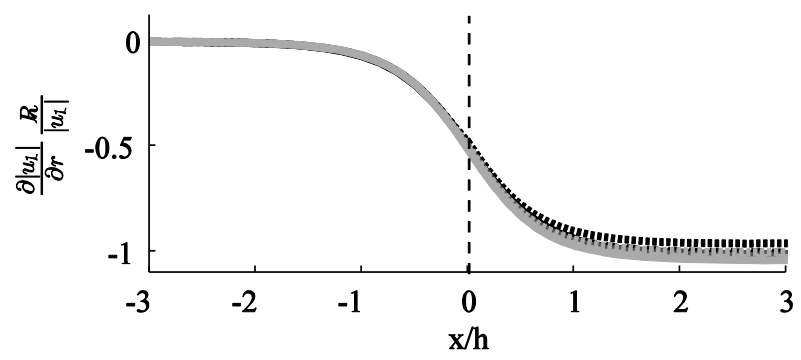

Figure 5.21: Cross-directional gradient in axial velocity scaled with the radius divided by the velocity amplitude at the center of the pipe, over the $x$-axis scaled with the half spacing $h$. The light gray, dark gray and black lines correspond to a shear wave number of $s=20,40$ and 60 respectively. While the solid lines, dashed lines and pointed lines correspond to a dimensionless radius of $\chi=10,5$ and 3 respectively. The vertical dashed line indicates the transition from straight to bend.

The scaling in Figure 5.21 shows that the transition length can be estimated as a function of the half spacing $h$. The transition region is defined as the length until the value of the scaled velocity derivative is reached with a tolerance of 0.01 . The resulting transition length $x_{t r}$ is then:

$$
x_{t r}=h \cdot 3.1
$$

This is the distance that the heat exchangers should be away of the bend such that the transitions due to the bend have no influences on the heat exchangers. This scaling is valid for the investigated shear wave numbers $s<20$. It is expected that this is no longer true when the half spacing is on the order of the viscous penetration depth, as the viscous effects becomes dominant then.

The fact that the cross-directional gradient in the axial velocity profile is not zero in the entire length of the straight section suggests that the cross-directional gradient in the pressure profile is not zero either. This has also been found to be the case, but the pressure gradient is too small in magnitude for accurate numeric differentiation. It is chosen instead to look at the cross-directional velocity, which is proportional to the crossdirectional gradient of the pressure profile.

Figure 5.22 shows the normalized maximum cross-directional velocity over the normalized axial coordinate, for the same geometries as those given in the earlier figures. The maximum cross-directional velocity is scaled with the axial velocity amplitude at the center line $\left.\left|u_{1}\right|\right|_{r=0}$ : 


$$
v^{\prime}=\frac{\max \left(v_{1}\right)}{\left.\left|u_{1}\right|\right|_{r=0}}
$$

Outside of the transition region the cross-directional velocity is negligible, while in the transition region the cross-directional velocity becomes important. The flow in crossdirection is needed as the volume flow rate stays constant and the axial velocity profile changes from the profile in a straight pipe to the one in a bent pipe. With increasing bend sharpness the cross-directional velocity in the transition region becomes more significant and reaches up to $10 \%$ of the axial velocity in the simulated cases. These radial velocities have to be taken into consideration when designing a thermoacoustic device, as they can affect close by components, like heat exchangers, which would reduce the efficiency of the device.

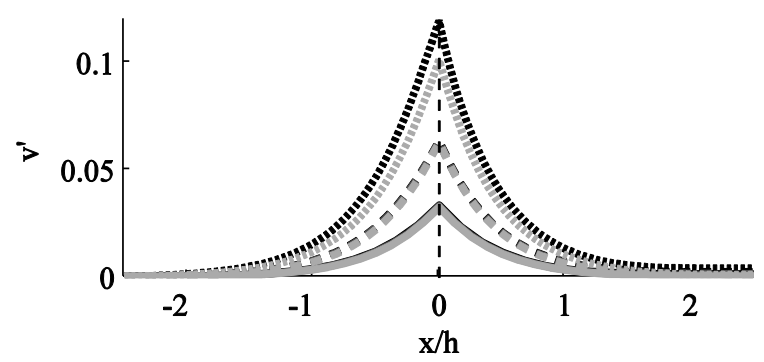

Figure 5.22: Normalized cross-directional velocity $v^{\prime}$ as a function of the scaled axial coordinate. The light gray, dark gray and black lines correspond to a shear wave number of $s=20,40$ and 60 respectively. While the solid lines, dashed lines and pointed lines correspond to a dimensionless radius of $\chi=10,5$ and 3 respectively. The vertical dashed line indicates the transition from straight to bend.

The influence of the bend radius is illustrated in Figure 5.23 by scaling the crossdirectional velocity by the axial velocity amplitude and the dimensionless bend radius $\chi$ :

$$
v^{\prime \prime}=\frac{\max \left(v_{1}\right)}{\left.\left|u_{1}\right|\right|_{r=0}} \chi
$$

For all the shown cases the lines lay over each other and the transition length is the same as for the axial velocity shown earlier. 


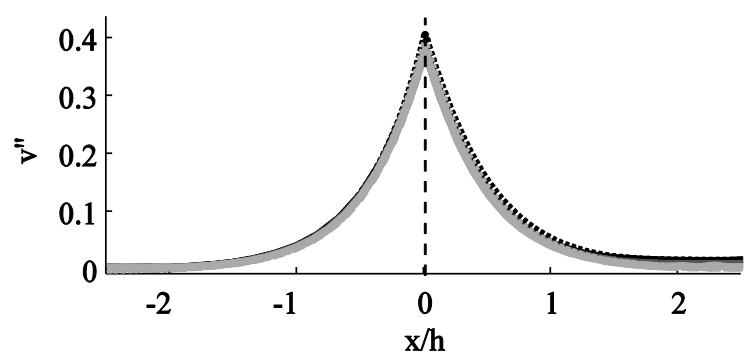

Figure 5.23: Normalized cross-directional velocity $v^{\prime \prime}$ over the axial coordinate normalized with the half spacing $h$. The light gray, dark gray and black lines correspond to a shear wave number of $s=20,40$ and 60 respectively. While the solid lines, dashed lines and pointed lines correspond to a dimensionless radius of $\chi=10,5$ and 3 respectively. The vertical dashed line indicates the transition from straight to bend.

The transition effects introduce additional losses that can be characterized by comparing with the low reduced frequency solution of a straight pipe. In Figure 5.24 the changes in acoustic power due to the bend are normalized with the changes of acoustic power obtained from the low reduced frequency solution:

$$
q^{\prime}=\frac{\frac{\partial E_{a c, \text { sim }}}{\partial x}-\frac{\partial E_{a c, \text { anal }}}{\partial x}}{\frac{\partial E_{a c, \text { anal }}}{\partial x}}
$$

For the cases where the transition region was the largest, which means that they have a low dimensionless bend radius and a high shear wave number, the bend introduces up to $3 \%$ additional losses per length compared to a straight pipe.

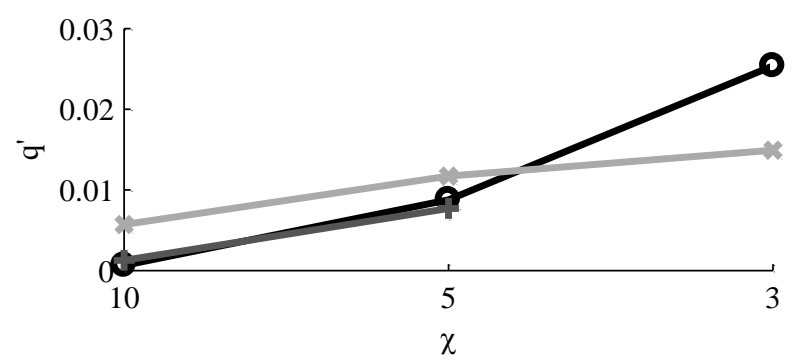

Figure 5.24: Additional dissipated acoustic power per length in the simulated cases with respect to the dissipated acoustic power per length predicted in the analytic model for shear wave numbers of 20, 40 and 60, in light gray, dark gray and black respectively.

The conclusion that can be drawn from this section is that the transition effects play an important role in the acoustic flow field when the dimensionless bend radius becomes lower. The length over which these transitions take place is defined by the half spacing. 
Geometric configurations where the transition effects begin to overlap in the round sections are found for high shear wave numbers and low dimensionless bend radii. In these short bends the cross-directional velocity is higher and the axial velocity is reduced.

Heat exchangers should be placed outside of the transition area and thus further away than $3.1 \cdot h$ from the bend. Otherwise the heat exchanger will be subject to an asymmetric axial velocity profile and an increased cross-directional velocity, decreasing the heat transfer in the heat exchangers.

\subsubsection{Limits of the analytical solution}

In the previous sections the reflections induced by the bend and the transition effects between the bend and the straight part have been investigated. In this section the validity of the low reduced frequency approximations is determined. Knowing the limits of validity of the low reduced frequency approximations is of interest as the approximation allows a quick and easy estimation of the flow field and acoustic power losses in the corresponding component, compared with CFD.

The assumptions made in order to derive the low reduced frequency solution were discussed in detail in Chapter 2.3 and will be shortly summarized in this chapter. The main assumption is that only small steady harmonic fluctuations occur in a pipe with a hydraulic radius much smaller than the wavelength: a low reduced frequency. When this assumption is valid the following characteristics used for the derivation of the analytical solution have to be true too:

- $\quad$ axial velocity is considerably larger than the radial velocity

$$
|u| \gg|v|
$$

- $\quad$ the pressure is constant over the height

$$
\frac{\partial p}{\partial r}=0
$$

- the gradient of axial velocity in the wave propagation direction is considerably smaller than the gradient of the axial velocity in radial direction

$$
\left|\frac{\partial u}{\partial x}\right| \ll\left|\frac{\partial u}{\partial r}\right|
$$

- the gradient of temperature in the wave propagation direction is considerably smaller than the gradient of the temperature in the radial direction

$$
\left|\frac{\partial T}{\partial x}\right| \ll\left|\frac{\partial T}{\partial r}\right|
$$

If the flow field does not fulfil the characteristics summarized in Equation (5.36) to (5.39) then the assumptions made are also not valid and the model is no longer a good approximation. The influence of the bend curvature on these assumptions is investigated here by discussing each equation individually in the next paragraphs, starting with the first Equation (5.36). 
In the previous section it is shown that a transition between bend and straight part introduces a cross-directional velocity component. The magnitude of this cross-directional component increases for decreasing values of the dimensionless bend radius. It is also pointed out that when the transition length becomes too large or when the bend length becomes too short, the two transition zones coincide and overlay each other. Both effects introduce a cross-directional velocity component which can become significant in the entire bent domain. This makes the assumption that the cross-directional component of the velocity is much smaller than the axial component invalid.

When both transition zones overlay each other, the cross-directional component becomes important. This is the case when the inner circumference is smaller than twice the entrance length:

$$
\frac{R-h}{\pi}<2 \cdot x_{t r}
$$

which is the same as:

$$
x<3
$$

This means that for a dimensionless radius $\chi$ smaller than three, the transition zones overlay and an important cross-directional velocity can be expected.

The introduction of a cross-directional component in the axial momentum equation causes terms that were discarded in the derivations of the low reduced frequency approximation to become significant. This leads to momentum transport in the cross-directional direction, thus reducing the amplitude of the axial velocity. The axial velocity is scaled again according to Equation (5.31). The results for shear wave numbers of 20, 40 and 60 and dimensionless radius of 5 and 3 are shown in Figure 5.25.

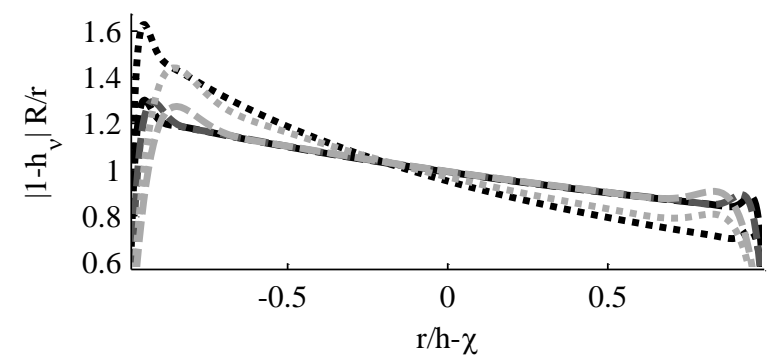

Figure 5.25: Scaled simulated axial velocity for low dimensionless bend radii and three shear wave number cases. The light gray, dark gray and black lines correspond to a shear wave number of $s=20,40$ and 60 respectively. While the dashed lines and dotted lines correspond to a dimensionless radius of $\chi=5$ and 3 , respectively.

The axial velocity of the cases with a dimensionless radius of $\chi=5$ overlay each other outside of the boundary layer. Furthermore, the profiles deviate by less than $1 \%$ from one in the center of the domain. In the case of a dimensionless radius of $\chi=3$ a larger deviation occurs, the curves do not overlay with each other anymore outside the boundary 
layer and the values at the center differ from one. This confirms the condition derived in Equation (5.41) from which dimensionless radius on the low reduced frequency approximation is no longer valid. In this case, the cross-directional velocity is not negligible compared to the axial velocity, which leads to a different axial velocity profile.

In the next step, the validity of Equation (5.37) is investigated, which states that the gradient of the pressure in the cross-direction is zero. In all simulations this gradient is very small and is much smaller than the axial pressure gradient. The characteristic given in Equation (5.37) is derived from the dimensionless radial momentum equation. Comparing the respective terms in the radial momentum equation obtained from the simulation it can be concluded that Equation (5.37) is still valid.

The third assumption given in Equation (5.38) states that the axial gradient of the axial velocity is much smaller than the radial gradient of the axial velocity. This also remains valid, as the largest radial gradients are still found in the boundary layer which is unaffected by the transition area.

The last assumption, that the temperature gradient in the radial direction is much larger than that in the axial direction, also stays valid, as the radial temperature gradients are still dictated by the boundary layer which, similar to the axial velocity, remains unaffected. This can be shown from the simulation results by looking at the thermal profile $\left|1-h_{\kappa}\right|$ over the cross-direction, shown in Figure 5.26. In this figure all the lines with equal shear wave number overlay each other perfectly and no influence of curvature on the thermal profiles can be seen.

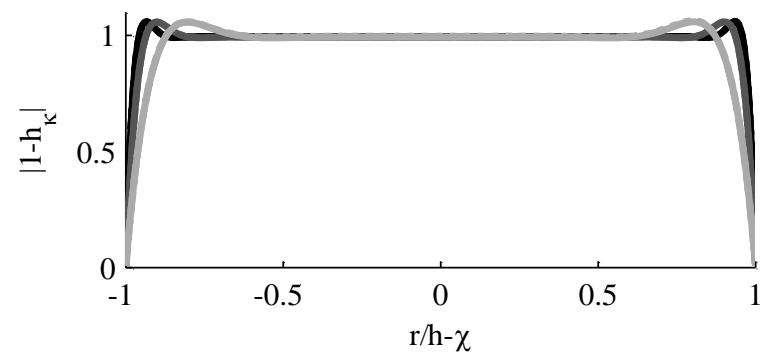

Figure 5.26: Scaled temperature profile in the middle of the bend over the dimensionless height. The light gray, dark gray and black lines correspond to a shear wave number of $s=20,40$ and 60 respectively. While the solid lines, dashed lines and pointed lines correspond to a dimensionless radius of $\chi=$ 10,5 and 3 , respectively.

From the previous investigation it can be concluded, that the assumptions used in the low reduced frequency solution hold for bends with a dimensionless radius $\chi>3$. For sharper bends it is found that the first assumption, which states that the axial velocity is much smaller than the radial velocity, does not hold anymore due to the transition effects from straight to bent pipe. The failure of this assumption primarily has an effect on the axial momentum equation, which leads to different axial velocity amplitudes and profiles. 
The axial momentum is converted into radial momentum and higher order effects in the transition area. This reduces the acoustic power, which was already reported in Figure 5.24 in the previous section. In this figure the underestimation of the dissipated acoustic power was found to be smaller than $1 \%$ for dimensionless bend radii of $\chi>3$. For sharper bends the approximation of the acoustic power deviates more.

This means that that the acoustic power dissipation can be approximated from the low reduced frequency approximation when the dimensionless bend radius is $\chi>3$. For sharper bends the errors become increasingly important, which makes the low reduced frequency solution invalid.

\subsubsection{Flow field for extreme curvatures}

In the previous section it was shown that for increasing sharp bends the velocity in the cross-directional direction becomes more and more important, leading to a deviation from the low reduced frequency solution. In this chapter even higher bend curvatures $(\chi<1.1)$ are discussed, as they appear when thermoacoustic devices are made compact. In the limit, the curvature is defined by the wall thickness of the pipe. In cases where the inner bend radius becomes on the order of the particle displacement amplitude, additional physical effects like flow detachment and subsequently vortex shedding occur.

Three parameters are involved in flow detachment in a bend for oscillatory flow. The first two were introduced in the previous sections, the dimensionless bend radius $\chi$ and the shear wave number $s$. The third dimensionless parameter is chosen to be the ratio of the inner radius $R_{i}=R-h$ and twice the particle displacement amplitude $\xi_{1}$. When this is rewritten, one finds that this equals the definition of the Strouhal number [109] multiplied by $\pi$. In the following this dimensionless quantity will be referred to as Strouhal number St:

$$
S t=\frac{R_{i}}{2 \xi_{1}}=\frac{\pi R_{i} f}{\left|u_{1}\right|}
$$

In general steady state flow fields, high Strouhal numbers are associated with viscosity dominated flow resulting in collective oscillating movements. For very low Strouhal numbers the high speed, quasi steady state portion of the flow is dominant. In intermediate regimes the oscillations are often characterized by vortex shedding [109]. In the simulations presented in the previous sections, collective oscillatory motion characterizes the flow, which is in accordance with the corresponding high Strouhal number. The Strouhal number corresponding to these cases is summarized in the Table 5.6.

In the results discussed up to now, all Strouhal numbers are larger than $1 / \pi$, which means that the particle displacement amplitude is smaller than the inner bend circumference. In these cases no vortex shedding occurs. For this reason additional simulations are presented now with a lower Strouhal number in order to reveal the limit for vortex shedding in a $180^{\circ}$ bend subject to an acoustic wave. An overview of the simulated cases with a low Strouhal number is given in Figure 5.27. Crosses indicate vortex generation and the 
circles mark the cases without vortex generation. The limit between vortex generation and no vortex generation lays at a Strouhal number $S t \approx 1$, which means that in order to create a vortex, the gas parcel has to displace over a distance that is at least three times the inner circumference of the bend.

Table 5.6: Strouhal number for the simulated cases in the previous section.

\begin{tabular}{c|cccc}
\hline \multirow{2}{*}{$\begin{array}{c}\text { Shear wave } \\
\text { number }\end{array}$} & \multicolumn{4}{|c}{ Dimensionless curvature } \\
& $\mathbf{1 . 5}$ & $\mathbf{3}$ & $\mathbf{5}$ & $\mathbf{1 0}$ \\
\hline $\mathbf{2 0}$ & 4.6 & 18.4 & 36.8 & 82.7 \\
$\mathbf{4 0}$ & 9.2 & 36.8 & 73.5 & 165 \\
$\mathbf{6 0}$ & 13.8 & 55.1 & 248 & 524 \\
\hline
\end{tabular}

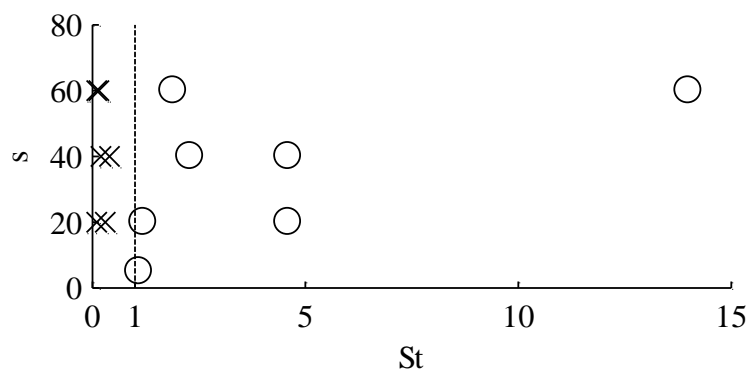

Figure 5.27: Vortex shedding in the performed simulations presented as a function of the shear wave number and the Strouhal number. Circles indicate simulations where no vortex shedding occurs, while crosses indicate simulations where vortex shedding occurs. Vertical dashed line indicate Strouhal Number $S t=1$.

Flow detachment is found to be the driving force behind the creation of vortices, which is visible in Figure 5.28. In this figure the velocity in the wave propagation direction for a shear wave number $s=40$ and a dimensionless bend radius of $\chi=1.01$ is plotted as a contour. The arrows in the figure show the normalized velocity vector. The plot is a detailed view at the location near the bend and shows the velocity field at the moment of maximum velocity in wave propagation $t=4.25 \cdot T$. The location where the flow detaches can be seen, as the velocity in wave propagation direction is zero there, and the velocity vector does not follow the curvature of the bend. This creates a recirculation zone, shown in blue. This behavior occurs in all the cases where vortex shedding appears.

The vortex generation occurs periodically, as the flow detaches twice per period, once at the bottom of the bend and once at the top when the flow direction is reversed. This is shown in Figure 5.29, where two contour plots of the velocity in wave propagation are 
shown at the moment of flow reversal a) $t=4.0 \cdot T$ and b) $\mathrm{t}=4.5 \cdot T$. The vectors show the normalized velocity vectors. At both times two vortices can be distinguished, one that was generated in the half period just finishing and the other one from the half period earlier. The vortices do not propagate and get dissipated within the following half period by the newly generated vortex. The previous implies that the vortices only occur in close proximity to the inner bend.

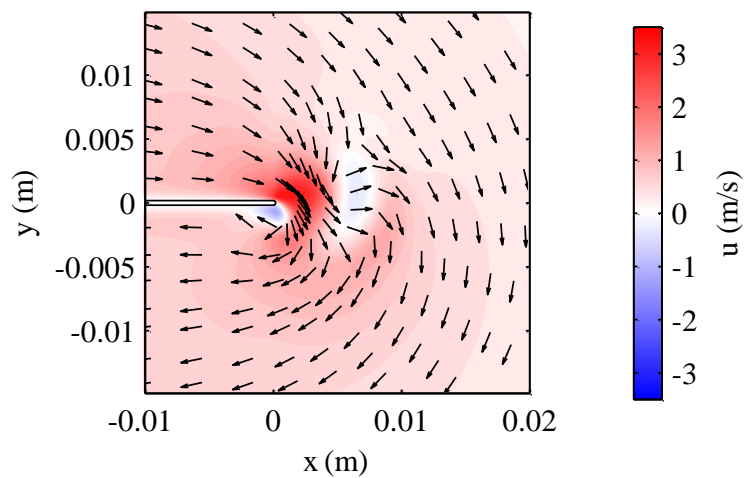

Figure 5.28: Contour of the velocity in wave propagation direction and normalized vector plots of the velocity at time $t=4.25 \cdot T$.

A short remark about the spatial discretization is given here, as the mesh was optimized for decoupled axial and cross-directional gradients in the acoustic flow field and this is not the case anymore. However, in the current configuration the mesh has the highest density in close proximity to the inner bend wall, where the vortex shedding occurs in the considered cases. Due to the fact that the gradients in the vortexes are lower than those occurring directly in the boundary layer, and in the current simulations do not propagate far inside the domain, it can be assumed that the mesh is fine enough to approximate the gradients accurately. At higher pressure amplitudes the vortices are expected to propagate away from the location where they are created. This means that in order to simulate higher amplitudes a mesh study has to be conducted which incorporates this new physical effect.

When designing a thermoacoustic device, the acoustic power dissipation is a quantity of interest. But in order to accurately quantify the acoustic power losses from simulation data, the first order effects need to be steady periodic in time. In the simulations with low dimensionless radius $\chi$, only 5 periods could be simulated, because of the high simulation time required, which in the most extreme cases corresponds to a simulation time of up to a month. Due to the vortex generation at dimensionless radii $\chi<1$, the flow field is not yet steady periodic, which makes it impossible to determine the first order effects accurately and with this it is impossible the quantify the additional acoustic power dissipation due to the flow separation. Generally one can say that extra acoustic power is dissipated as a result of energy transfer to higher harmonics and vortexes, in situations with extreme 
curvatures. This happens on top of the regular viscous dissipation caused by the boundary layer.

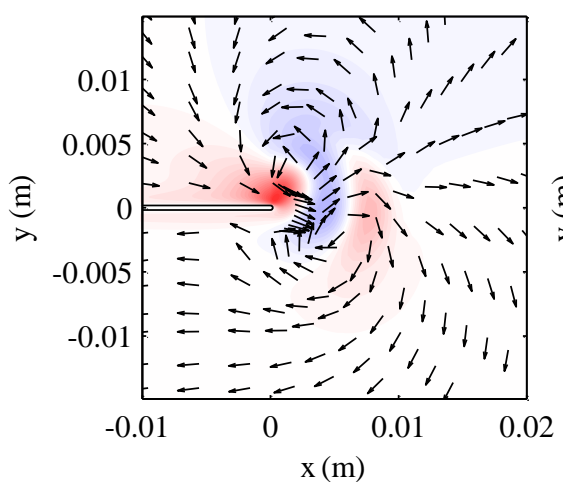

a) $t=4.0 \cdot T$

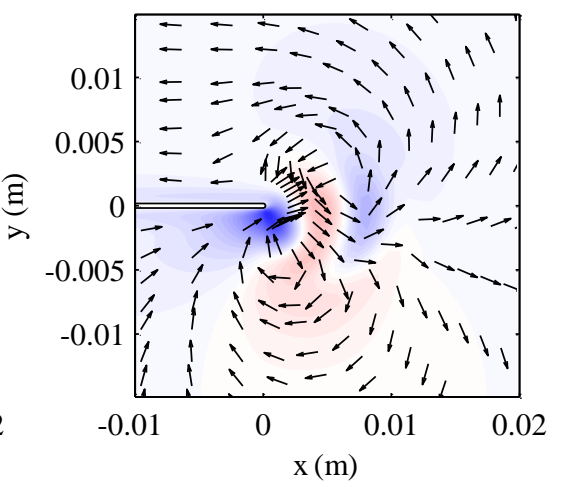

b) $t=4.5 \cdot T$

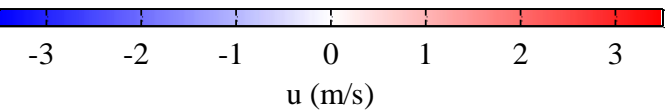

Figure 5.29: Contour plot of the velocity in wave propagation direction, with normalized vector plots of the velocity at the two moments where flow reversal occurs. At both time steps two vortices can be distinguished.

\subsubsection{Conclusion and recommendation}

In this chapter the flow field in a $180^{\circ}$ curved layer was investigated. The problem can be parametrized with three dimensionless quantities: the shear wave number $s$, the dimensionless radius $\chi$ and the Strouhal number St. The present study focuses on the influence of the geometry, which means that the first two parameters were investigated.

It is shown that with decreasing dimensionless radius $\chi$, which corresponds to sharper bends, the reflection due to the bend increases. The reflections in the investigated parameter range do not exceed $10 \%$ for all cases and have to be taken into account in the design of thermoacoustic engines in order to reach reliable predictions and high efficiencies. The second parameter, the shear wave number, has no influence on the reflections in the investigated parameter range.

In the next step the limit of the low reduced frequency approximation was determined by analyzing the transition effects from the straight to the bent part of the pipe. For large dimensionless radii, $\chi>3$, the transition effects at both extremities of the bend do not interact, the cross-directional velocity is small and the low reduced frequency solution is valid. At smaller dimensionless radii $(\chi<3)$ the transition effects from both extremities interact and the cross-directional velocity becomes increasingly important for sharper bends, making the low reduced frequency approximation invalid. This is also accompa- 
nied by an increase in acoustic power dissipation which has to be taken into account in the design of thermoacoustic engines.

For dimensionless bend radii even smaller, which are on the order of the displacement amplitude, vortex generation is observed. It is shown that in the investigated parameter range for Strouhal numbers $S t<1$, vortices occur, that are expected to introduce additional losses.

In future studies the parameter space spanned by the shear wave number $s$ and the dimensionless radius $\chi$, has to be extended by the Strouhal number $S t$ such that the influence of the pressure amplitude is also taken into account. Higher pressure amplitudes would imply that the vortices would not only dissipate but also propagate outside of the bend. This would involve a different mesh to resolve the vortex propagation and longer simulation to reach a steady periodic state. Additionally, the inlets at both ends of the domain have to be extended such that the vortices do not influence the non-reflecting boundary conditions.

As Dequand et al. [104] showed that the shape of the outer radius has an influence on the acoustic field, the bent geometry of the CFD model should be varied in future studies. A geometry of interest would be for example the coaxial configuration with a flow redirection plate, as this is applied in compact thermoacoustic devices. In an additional step the model could be extended to the third dimension, especially in order to investigate pipes with a circular cross-section, which is expected to have influence on the vortex generation in the $180^{\circ}$ bend.

In a subsequent step the interaction of the bend with a close by component, like a heat exchanger, could be investigated by implementing the component in the CFD model. This is especially interesting as reduced models cannot predict this kind of interactions. 


\subsection{Calculation of thermoacoustic functions with CFD}

Thermoacoustic engines are designed using the one-dimensional thermoacoustic equations introduced in Chapter 2.2.3. In these equations the thermal and viscous thermoacoustic functions, $f_{\kappa}$ and $f_{v}$ respectively, allow accounting for the three-dimensional thermal and viscous effects in the one-dimensional thermoacoustic equations. This means that by knowing the thermoacoustic function, the time dependent three-dimensional flow field can be reduced to a one-dimensional frequency dependent problem.

The thermoacoustic functions for uniform pores can be obtained by solving the differential equation

$$
h_{j}-\frac{\delta_{j}^{2} i}{2} \nabla_{c d}^{2} h_{j}=0, \quad \text { for } j=\kappa \text { or } v
$$

and integrating the resulting function $h_{j}$ over the cross-section

$$
f_{j}=\iint_{A} h_{j} d A, \quad \text { for } j=\kappa \text { or } v
$$

For simple geometries analytical solutions can be derived. Rott [5] derived analytical solutions of the thermoacoustic functions for parallel plates and cylindrical pores, while Arnott et al. [52] extended the analytical solutions for other uniform pores. The thermoacoustic functions of the most important pore geometries are summarized in Appendix B and visualized in Chapter 2.2.4.

However, many thermoacoustic devices comprise stacked screens as the regenerator. For these regenerators no analytical solution for the thermoacoustic functions is known. Swift and Ward [110] used steady flow correlations to approximate the thermoacoustic functions for these tortuous regenerators, which do not take into account the flow history. Nevertheless, this allows implementing complex geometries in thermoacoustic codes like DeltaEC [7].

Experimental characterization of the thermoacoustic functions has been done by different researchers. Hayden and Swift [54] calculated the thermal thermoacoustic function of a pin array stack using the relation given in Equation (2.46) by measuring the pressure and the volume in their experimental setup. Wilen investigated a single pore [111] and measured the thermal thermoacoustic function of a single pore [112]. In a later work Wilen and Petculescu extended the study to a single pore with a temperature gradient [113] and to high pressure amplitudes [114]. They also measured the pressure and the volume of the experimental setup in order to estimate the thermal thermoacoustic function. Only by similitude the viscous thermoacoustic function can be estimated. Udea et al. [56] extended the experiments such that the full thermoacoustic transfer matrix can be obtained, which allows the calculation of both thermoacoustic functions. The underlying idea is to model the regenerator as an array of tubes having a simple geometric crosssection. Recently, Bannwart et al. [115] proposed a more accurate measurement method that uses an acoustic impedance sensor to estimate the thermoacoustic transfer function. 
The only work known to the author in which the thermoacoustic functions are calculated from CFD is the work of Jensen and Raspet [55]. They use the Lattice Boltzmann method in order to reveal the flow around random fibrous material. From the flow field the thermal thermoacoustic function is calculated using the relation given in Equation (2.46). Similar to some of the experimental work mentioned earlier, the viscous thermoacoustic function has to be estimated by similitude from the thermal thermoacoustic function.

CFD simulations which are used to estimate the thermoacoustic functions of a stacked screen regenerator are not known to the author. The CFD simulations of stacked screens evaluate the characteristics of the regenerator for steady flow (a recent study is for example Costa et al. [116]). However, for thermoacoustic applications the phase delay is important. To incorporate this phase delay the complex heat and momentum transfer have to be determined.

In this chapter a first step is made to reveal the flow field inside a stacked screen regenerator subject to an oscillating flow. The CFD simulations in this chapter are the first CFD simulations that allow the calculation of the thermoacoustic transfer matrix and hence both thermoacoustic functions of stacked screen regenerators from the oscillating flow field. As the pressure and the velocity are available, the method of Udea et al. [56] will be used to calculate the thermoacoustic functions from the CFD simulations. Different geometries will be investigated and compared to one-dimensional models of the regenerator.

\subsubsection{Models}

In this section the models of the stacked screen regenerator are presented. A geometric reduction is done in order to reduce the computational costs. Assuming that the screens are perfectly inline, and neglecting both, the gravity effects as well as the effects of the duct where the regenerator is housed, one mesh can be modeled as a periodic repetition of one cell of the meshed screen. Each of these cells is further reduced to inline cylinders, where each cylinder represents the wire of a screen. The geometric reduction is summarized in Figure 5.30.

First in Section 5.3.1.1 the CFD model of the stacked screen regenerator is presented, then in Section 5.3.1.2 a one-dimensional model of the regenerator is presented, which allows explaining the behavior seen from the CFD simulations. 


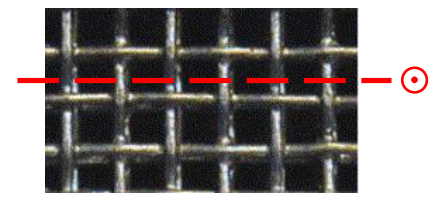

a)

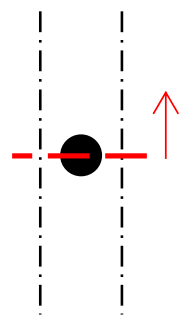

b)

Figure 5.30: Visualization of geometric reduction. a) full screen b) reduced CFD model. Red arrow indicates the wave propagation direction.

\subsubsection{CFD Model}

In this section the CFD model of the stacked screens is presented. Helium is used as a working gas, as it is frequently used in thermoacoustic engines for its favorable thermoacoustic properties [5]. Helium is modeled as an ideal gas with the fluid parameters given in Appendix E. The simulations are conducted at atmospheric pressure and a mean temperature of $T_{0}=300 \mathrm{~K}$. The wave is introduced at the left boundary through a nonreflecting boundary condition. It travels through the domain where it interacts with the cylinders representing the screen of the regenerator. Depending on the investigated case 1, 2, 5 or 10 screens are modeled. The boundary conditions at the cylinders modeling the screens are set to isothermal and no-slip. The wave leaves the domain at the right through another non-reflecting boundary condition. All boundary conditions are summarized in Figure 5.31.

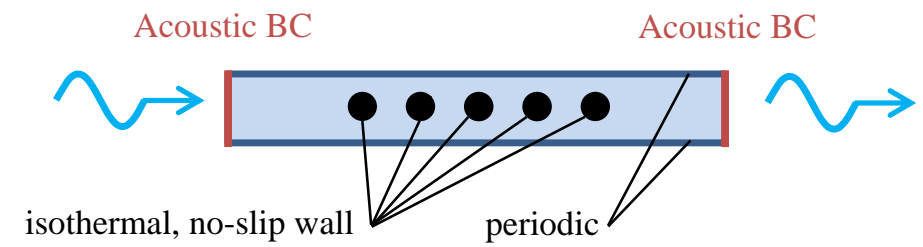

Figure 5.31: Summary of the boundary conditions applied in the stacked screen regenerator model.

The frequency of the wave is varied from $f=50 . .1300 \mathrm{~Hz}$. This corresponds to a Lautrec number of $L c=r_{h} / \delta_{\kappa} \approx 0.3 . .2$, which is the range used for stacks and regenerators in thermoacoustic devices. The pressure amplitude is set to $p_{1}=1 \mathrm{~Pa}$, which is considered to be a very low value for thermoacoustic applications, but allows a first estimation of the thermoacoustic functions of the stacked screen regenerator model.

The diameter of the cylinders corresponds roughly to the thermal penetration depth at $f=100 \mathrm{~Hz}$. In the reference geometry the opening of the screens is set to $2 \cdot h=\delta_{\kappa}$. The 
inlet and outlet length $L_{1}$ is twelve thermal penetration depths long, such that the distance between the screen and the boundary condition is always larger than two displacement amplitudes. The distance between the screens $L_{1}$ is one thermal penetration depth. The dimensions of the stacked screen regenerator model are summarized in Figure 5.32.

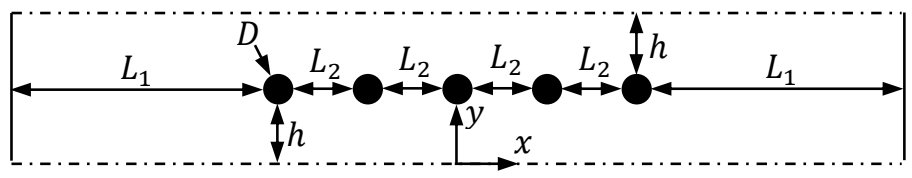

Figure 5.32: Definition of the dimensions of the thermoacoustic function CFD model.

The ratio of the cylinder diameter and the viscos penetration depth $D / \delta_{v}$ is smaller than four for all simulations and the acoustic Reynolds numbers $R e_{a c} \approx 49$ for all simulations, such that the flow can be assumed laminar [84].

The fluid domain is discretized with an O-grid around the cylinders and a rectangular mesh at the inlet and outlet of the domain. The discretization in the radial direction consists of 30 elements growing with a geometric factor of $q=1.2$ away from the cylinders. This is more than the minimum number of elements per extended viscous penetration depth derived in Chapter 4.1.2.1. The circumference of the cylinders is discretized with 120 elements. The inlet and the outlet lengths are discretized with five elements per thermal penetration depth $\delta_{\kappa}$ in the wave propagation direction, which corresponds to at least 5700 elements per wave length. The cross-direction is discretized with twenty elements per thermal penetration depth $\delta_{\kappa}$. The mesh of the five screens model is shown in Figure 5.33 as an illustration.

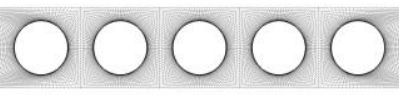

Figure 5.33: Discretization of the 5 screen mesh model.

The domain is initialized with zero velocity and a temperature of $T_{0}=300 \mathrm{~K}$. All simulations are carried out for two wave periods. The time step size is limited by the nonreflecting boundary condition and is set to $\Delta t=5 \cdot 10^{-7} \mathrm{~s}$, which corresponds to at least 1500 time steps per period.

\subsubsection{One-dimensional model}

The CFD simulations are compared to a one-dimensional model based on the wave propagation equations derived in Chapter 2.2.4.3.2. By adapting the one-dimensional model the different characteristics of the CFD results can be easily interpreted. In the one-dimensional model the cylinders that represent the wires of the regenerator are mod- 
eled as parallel plates, with comparable properties. The half spacing needed for the parallel plates is evaluated as the averaged half spacing of a cylinder:

$$
h_{p p}=\frac{H}{2}-\frac{\pi \mathrm{R}}{4}
$$

where the geometric parameters are defined in Figure 5.34.

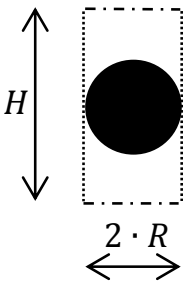

a)

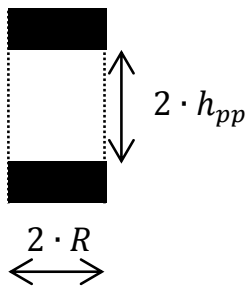

b)

Figure 5.34: a) cylinder modeling the stacked screens and b) the corresponding simplified parallel plate geometry with the definition of the corresponding dimension.

The resulting geometric reduction from a) the cylinder to b) the parallel plate models is shown in Figure 5.35.

a)

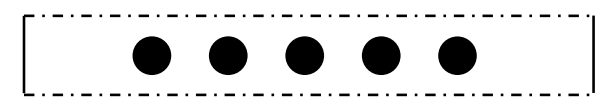

b)

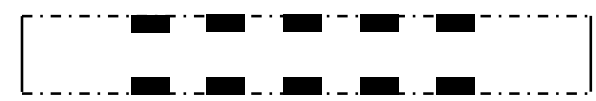

Figure 5.35: a) Simulation model: cylinders where the flow goes around b) acoustic model of the simulation.

The regenerator model is then split into different segments of length $\Delta x$ for which the pressure $p_{i+1}$ and the velocity $u_{i+1}$ on one side of the segment can be calculated from the pressure $p_{i}$ and velocity $u_{i}$ on the other side as follows:

$$
\begin{gathered}
p_{i+1}=p_{i} \cos (k \cdot \Delta x)-i Z u_{i} \sin (k \cdot \Delta x) \\
u_{i+1}=u_{i} \cos (k \cdot \Delta x)-i \frac{p_{i}}{Z} \sin (k \cdot \Delta x)
\end{gathered}
$$

Each segment is characterized by its acoustic impedance $Z$ and its wave propagation constant $k$. Three different types of segments can be differentiated. These segments are visualized in Figure 5.36. In the first type of segment (a) the wave propagates adiabatically, the acoustic properties are set as follows:

$$
\begin{gathered}
k=k_{0}=\frac{\omega}{c_{0}} \\
Z=Z_{0}=c_{0} \rho_{0}
\end{gathered}
$$


in the second type of segment (b) the wave interacts with the screen. According to the geometric reduction given above, the acoustic properties can be estimated from the thermoacoustic functions as follows:

$$
\begin{gathered}
k=\frac{\omega}{c_{0}} \sqrt{\frac{1+(\gamma-1) f_{\kappa}}{1-f_{v}}} \\
Z=\frac{\omega \rho_{0}}{k\left(1-f_{v}\right)}
\end{gathered}
$$

The thermoacoustic functions are calculated from the analytical solutions for parallel plates using the half spacing $h$ defined in Equation (5.45)

The last type of segments (c) models the wave propagation in between the screens. The wave propagation is modeled as adiabatic even though that the gas interacts also with the screen in this volume. These axial effects of the screens are taken into account in the model by implementing the thermal hysteresis losses at the vertical surfaces. The velocity at this point is corrected as follows:

$$
u_{i+1, \text { cor }}=u_{i+1}-\frac{1}{2} \omega l_{1} \frac{\gamma-1}{\rho_{0} c_{0}^{2}}(1-\epsilon) p_{i}
$$

where $l_{1}$ is a geometric length which is generally the thermal penetration depth $\delta_{\kappa}$. Due to the close packaging of the screens, the distance between two screens in the wave propagation direction can be smaller than two thermal penetration depth $\delta_{\kappa}$. For this reason the length $l_{1}$ is limited to the distance between the cylinders for low frequencies.

The pressure and the velocity at the two ends of the domain are then used to calculate the thermoacoustic function of the whole assembly as is done for the simulations.

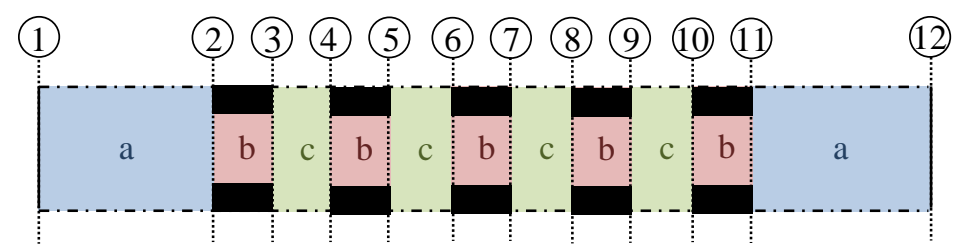

Figure 5.36: Different parts of the acoustic model: a adiabatic part; $b$ part with flow between parallel plates; $\mathrm{c}$ part between the parallel plates.

\subsubsection{Results and discussion}

In this chapter the thermoacoustic functions calculated from the reduced CFD model are presented. The results from the CFD models are compared to the results of the onedimensional model and the results for parallel plates, to allow for a better interpretation of the results.

Figure 5.38 shows the typical results that can be obtained from the calculation of the thermoacoustic functions. The viscous and thermal thermoacoustic functions are shown in a) and b), respectively, and are split into their real part (top) and imaginary part (bot- 
tom). The thermoacoustic functions are shown as a function of the Lautrec number $L c$, which is in fact, the dimensionless square root of the frequency, as the fluid parameters and the geometry are not changed for a range of simulation. The Lautrec number is the ratio of a characteristic length and the respective penetration depth. The typical length scale is the hydraulic radius [6] and it will be used in this work for thermal thermoacoustic functions. The hydraulic radius is calculated as follows:

$$
r_{h}=\frac{H}{\pi}-\frac{1}{2} R
$$

In the case of the viscous thermoacoustic function, the frequency is scaled with the minimum half spacing $h$ (see Figure 5.37), as this shows a better agreement with existing analytical solutions.

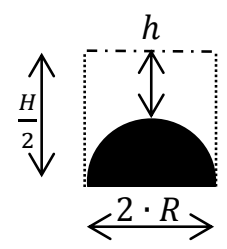

Figure 5.37: Definition of the lengths for the calculation of the Lautrec number.

A motivation for this different scaling is given in Figure 5.39. The contour plots of the velocity a) and the temperature b) for the case with a frequency $f=1300 \mathrm{~Hz}$ are shown at the moment of their maximum. While the temperature changes take place in a radial direction around the cylinders, the velocity shows a more "pore-like" structure in the wave propagation direction. This means that the temperature changes take place in the whole fluid volume and that the hydraulic radius is an appropriate scaling length. In the case of the velocity only a small channel, with a height of roughly the opening of the mesh incorporates the flow, the corresponding scaling length is thus the half spacing. Qualitatively similar results are also obtained for the other simulated frequencies.

This difference in scaling is remarkable as a number of publications only estimate the thermal thermoacoustic function and scale these results in order to obtain the viscous one $[55,111,112,113,114]$. According to the results shown in Figure 5.38, this does not seem to be true for non-uniform pores modeling a regenerator. A method like the one of Udea et al. [56] that is applied in this thesis should be used to calculate both thermoacoustic functions at the same time.

In Figure 5.38 the thermoacoustic functions are shown for different scaling of the domain, the corresponding cylinder diameter is given in the legend. All results lie on a continuous line and overlay each other in case of equal Lautrec numbers. From this it can be concluded that the numerical domain can be scaled without loss of generality when no vortexes or turbulence occurs. 

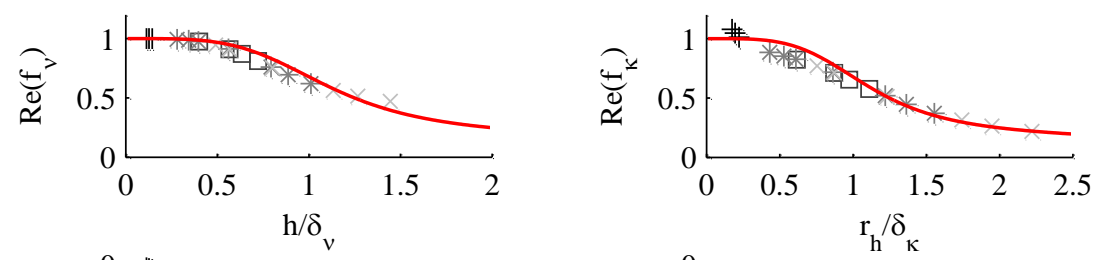

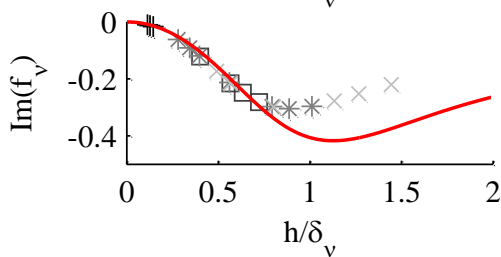

a)

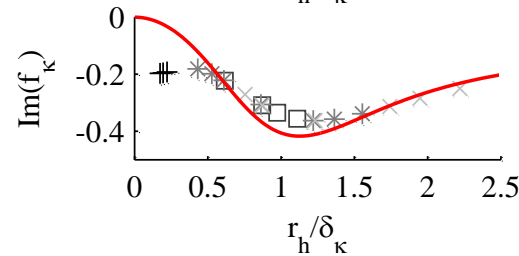

b)

$$
\begin{aligned}
& +\quad \mathrm{D}=1 \cdot 10^{-4} \mathrm{~m} \\
& \square \quad \mathrm{D}=5 \cdot 10^{-4} \mathrm{~m}
\end{aligned}
$$

$$
\begin{aligned}
& \text { * } \quad \mathrm{D}=7 \cdot 10^{-4} \mathrm{~m} \\
& \times \quad \mathrm{D}=1 \cdot 10^{-3} \mathrm{~m}
\end{aligned}
$$

Figure 5.38: Viscous a) and thermal b) thermoacoustic functions over the viscous Lautrec number $h / \delta_{v}$ and the Lautrec number $r_{h} / \delta_{\kappa}$ respectively for the ten cylinders CFD model. The geometry of the CFD model is scaled, such that four different diameters can be simulated. The red line shows the thermoacoustic function for parallel plate geometry as a function of the Lautrec numbers based on the hydraulic radius.

a)
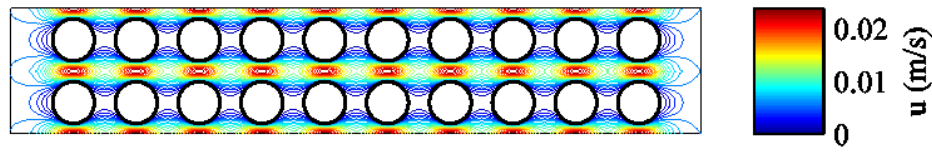

b)

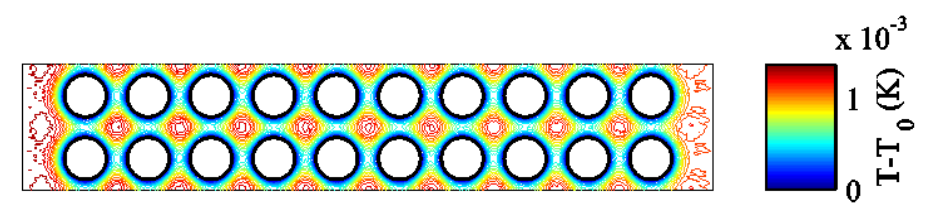

Figure 5.39: a) contour of the velocity and b) contour of the temperature at the moment of highest pressure and velocity, in the case of the simulation of $f=$ $1300 \mathrm{~Hz}$. Not the whole length of the computational domain is shown and the domain is repeated once along the periodic boundary condition. 
The thermoacoustic functions obtained from CFD are first compared to the thermoacoustic functions of the parallel plate geometry, which are plotted as red lines in Figure 5.38. Parallel plates are chosen as all uniform pores have a similar behavior and both the parallel plate geometry and the CFD model are two-dimensional. Deviation between the behavior of the parallel plate and the CFD model can be seen and will be explained using the one-dimensional model.

In Figure 5.40 the CFD results of Figure 5.38 are condensed to the thin blue line by connecting the respective points. The resulting curve that represents the results from the different scaling's is then compared to results of the one-dimensional model, which are represented by the thicker lines. In the one-dimensional model the thermal behavior of the regenerator model is adapted to distinguish different thermal effects. As expected these changes do not have any effect on the viscous thermoacoustic function shown in Figure 5.40 a), but only on the thermal thermoacoustic function shown in b), especially on its imaginary part. Hysteresis losses are implemented in the one-dimensional model in order to take into account the losses due to the heat transfer in the wave propagation direction. If no hysteresis losses are included in the one-dimensional model, the imaginary part of the thermal thermoacoustic function goes to zero for low Lautrec numbers, but if they are included, the thermal thermoacoustic function goes to a large negative value. The hysteresis losses thus introduce an additional phase delay. This is the same behavior that can be seen from the CFD results, but the effect is less pronounced for low Lautrec numbers and hence low frequencies. This can be explained by the fact that for low frequencies the distance between the cylinders is smaller than the thermal boundary layer, limiting the hysteresis losses. This is taken into account in the one-dimensional model by limiting the thermal penetration depth to a maximum value of half the distance between the cylinders. This means that for low frequencies the length $l_{1}$ in Equation (5.52) is set to half the distance between the cylinders $L_{2} / 2$. The results are given in Figure 5.40 with the gray dash dotted line and show a behavior close to the results from CFD. This means that it can be concluded from the one-dimensional model that the deviation in the imaginary part of the thermal thermoacoustic function is due to heat transfer in wave propagation direction.

Another remarkable qualitative difference occurs in the real part of the thermal thermoacoustic function, as it gets higher than one for low Lautrec numbers in case of the CFD data shown in Figure 5.38 b). This is unexpected as for all uniform pores the value is bounded between zero and one. One possible reason can be the inaccuracy in the calculation of the velocity phasing that leads to an erroneous calculation of the real part of the thermal thermoacoustic function. This effect is discussed in more details in Appendix I.

In Figure $5.40 \mathrm{~b}$ ) a difference in the real part of the thermal thermoacoustic function can be seen between the one-dimensional model and the CFD results. For low frequencies or low Lautrec numbers respectively the values of the one-dimensional model converge to a constant value, which is clearly smaller than in the CFD simulations. In Ap- 
pendix $\mathbf{J}$ it is shown that this effect can be explained by the fact that the space between two screens does not interact with the fluid in the one-dimensional model and a scaling is proposed there to demonstrate this. The values from CFD are clearly higher than those from the one-dimensional model, which indicates that the distance in the wave propagation direction in between the cylinders also takes part in the heat transfer. This effect is introduced in the one-dimensional model by assuming that in between the cylinders the thermal contact that occurs is similar to that of parallel plates with half spacing $h=H / 2$. The results are shown with the light gray dashed line. With this the behavior of the thermal thermoacoustic function obtained from CFD can be explained.

The behavior of the viscous thermoacoustic functions in Figure 5.38 a) shows less deviation from the parallel plate geometry than the thermal thermoacoustic function in $b$ ). In Figure 5.40 the results are compared to the one-dimensional model. For low frequencies or Lautrec numbers respectively a good agreement between the one-dimensional model and the CFD simulations are obtained. For higher Lautrec numbers the twodimensional geometry becomes increasingly important as the boundary layer becomes thinner and the displacement amplitude smaller. Thus, the deviations increase for higher Lautrec numbers.
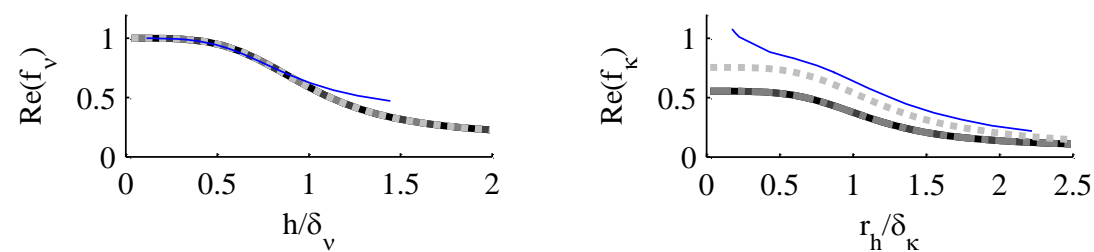

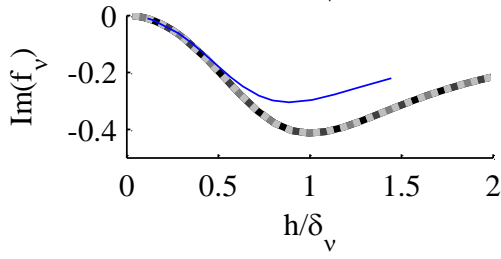

a)

No hysteresis losses

-ーーー Hysteresis losses

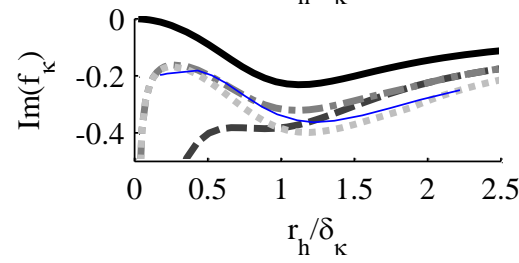

b)

- $\because=-$ Limited hysteresis losses

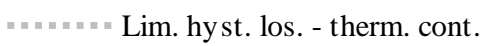

Figure 5.40: Viscous a) and thermal b) thermoacoustic functions over the viscous Lautrec number $h / \delta_{v}$ and the Lautrec number $r_{h} / \delta_{\kappa}$. The thin blue line represents the condensed CFD results from Figure 5.38. The different thick lines represent results from the one-dimensional model, with changing thermal properties.

In the previous the general behavior of thermoacoustic functions was described. In the following the geometry and the pressure amplitude are changed. In Section 5.3.2.1 the 
number of screens is varied, then in Section 5.3.2.2 the arrangement of the cylinders is changed and in Section 5.3.2.3 the influence of higher amplitudes is investigated.

\subsubsection{Influence of the number of screens}

In this section the influence of the number of screens on the estimated thermoacoustic functions is investigated. This means that regenerators with different lengths are compared such that the length over which the entrance effects at each side of the regenerator occur, can be estimated. Entrance effects occur at the extremities of the regenerator, as the gas parcels at the extremities interact with both the regenerator as well as with the open space. The entrance effects were already described in Chapter 4.6 and will be investigated in more details for stacked screen regenerators in Chapter 5.4.
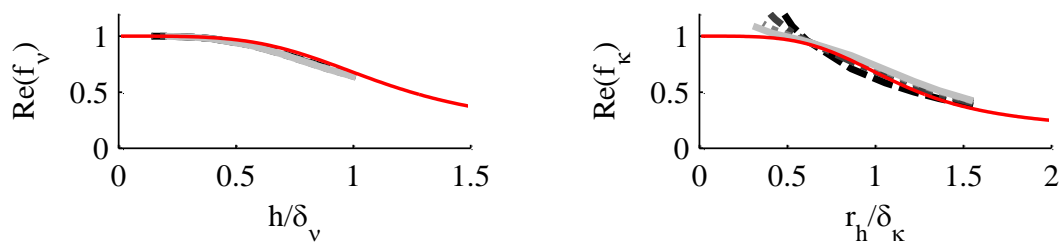

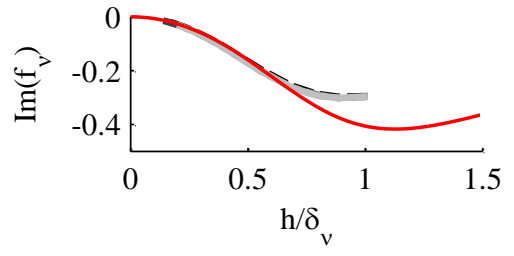

a)

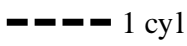

-"-r- 2 cyl

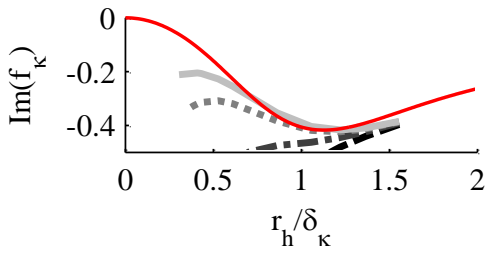

b)

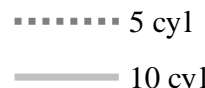

Figure 5.41: Viscous a) and thermal b) thermoacoustic functions over the viscous Lautrec number $h / \delta_{v}$ and the Lautrec number $r_{h} / \delta_{\kappa}$. The black and gray lines show the thermoacoustic functions for one, two, five and ten cylinders and the red line shows the thermoacoustic function for parallel plates.

The length of the stacked screen regenerator is adapted in the CFD simulations by varying the number of cylinders. The thermoacoustic functions for one, two, five and ten cylinders are shown in Figure 5.41. The behavior of the viscous thermoacoustic function is not affected by the number of cylinders, which means that viscous effects at the extremities of the regenerator do not play a role for this pressure amplitude. On the other hand, the behavior of the thermal thermoacoustic function is notably affected by the entrance effects. With an increasing number of cylinders the real and the imaginary part get closer to the corresponding ones of parallel plates. The entrance effects are averaged out for a larger number of cylinders, leading to a more pore like behavior. This can be seen especially at low frequencies, where the entrance effects have more influence due the 
larger displacement amplitude and boundary layer. Indeed, the hysteresis losses that affect the imaginary part of the thermal thermoacoustic function become less important and the value for low Lautrec numbers goes closer to zero. Also the overshoot of the real part of the thermal thermoacoustic function for low frequencies gets smaller with an increasing number of cylinders. This indicates that the values higher than one do not necessarily originate from numerical errors but could originate from the entrance effects. To show this the thermoacoustic functions of each cylinder are calculated in the following.

In order to calculate the thermoacoustic functions of each of the cylinders of the regenerator separately, the flow data is exported from the line between two cylinders and averaged over this cross-section. From the time dependent pressure and velocity, the thermoacoustic functions of each cylinder can be estimated with the method from Udea et al. described in Chapter 2.2.4.3. As can be expected from Figure 5.41 the values of the viscous thermoacoustic function per cylinder are the same for all cylinders, as the number of cylinders does not have any influence. As opposed to this, the values of the thermal thermoacoustic function per cylinder show a clear dependence on the entrance effects. The thermal thermoacoustic function of the first two cylinders at each end of the regenerator differs largely from the values of the cylinders further inside of the regenerator. The thermal thermoacoustic functions of cylinders more than two cylinders inside the regenerator overlay each other. This is illustrated in Figure 5.42, which shows the thermal thermoacoustic function for the cylinders at the center of the regenerator for the CFD simulations with five and ten cylinders. Both overlay each other, which means that the curves presented in Figure 5.42 are those of a stacked screen regenerator modeled as inline cylinders without entrance effects.

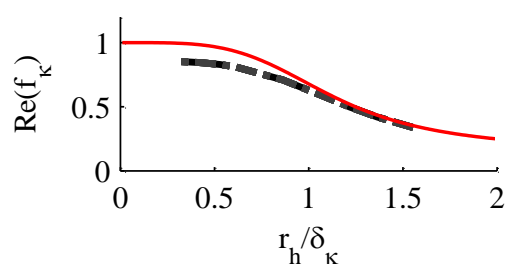

a)

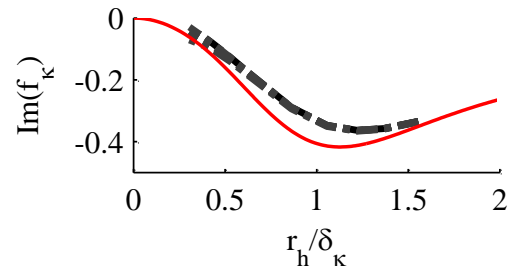

b)

Figure 5.42: a) real part and b) imaginary part of the thermal thermoacoustic functions of the cylinders in the center of the regenerator over the Lautrec number $r_{h} / \delta_{\kappa}$. The black and gray lines show the thermoacoustic functions of the cylinders in the five and ten cylinders case, colors are the same as in Figure 5.41. The red line shows the thermoacoustic function for parallel plates as an indication.

The behavior at low frequencies or low Lautrec number respectively is closer to that of parallel plates. For low frequencies the imaginary part of the thermal thermoacoustic functions goes to zero and the real part stays between zero and one. This is a further indi- 
cation that the values higher than one of the real part of the thermoacoustic function are due to the entrance effects.

Still the real part of the thermal thermoacoustic function shows a remarkable feature as it seems to converge to a value smaller than one for low frequencies. This indicates that the volume in between the cylinders does not take part at the thermal contact. For this reason the screens arrangement is changed in the next chapter in order to reveal the influence of the two-dimensional flow field on the thermal thermoacoustic function.

\subsubsection{Influence of the cylinder arrangements}

In the previous chapter it is shown, that the real part of the thermal thermoacoustic function does not go to one for low frequencies or Lautrec numbers respectively. This suggests that the entire fluid volume in between the cylinders takes part in the heat exchange. For this reason, the arrangement of the screens is adapted. Instead of an inline arrangement the screens are positioned in an alternating configuration. The new arrangement can be seen in Figure 5.45. The thermoacoustic functions are calculated cylinder wise, like in the previous chapter, and the values for the fifth cylinders are compared. While the viscous thermoacoustic function is not affected by the different arrangement, this is the case for the thermal one.

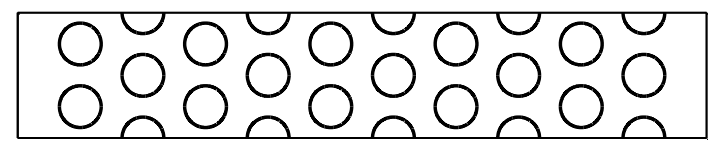

Figure 5.43: Alternating screen arrangement. Not the whole length of the computational domain is shown and the domain is repeated once along the periodic boundary condition.

Figure 5.44 shows the real part a) and the imaginary part b) of the thermal thermoacoustic function for the two different arrangements. The black and gray lines correspond to the inline and alternating arrangement respectively. The rearrangement leads to better thermal contact between the cylinders and the gas volume, as can be seen from the higher values of the real part of the thermoacoustic function for all frequencies. This different behavior is due to the fact that the maximum distance of the gas parcels to a cylinder is smaller in the alternating arrangement. Figure 5.45 shows the velocity in the wave propagation direction and the temperature at the moment of highest amplitude for the alternating arrangement and can be compared to the inline arrangement in Figure 5.39. Indeed the temperature profile in the alternating case is more homogeneous, which indicates that the temperature changes are smaller, the thermal contact is thus closer.

Finally, it can be concluded that rearranging the cylinders does not affect the viscous behavior of a stacked screen but can be advantageous from a thermal point of view. The best arrangement is reached when the screens are positioned such that they have the closest contact with the fluid. 


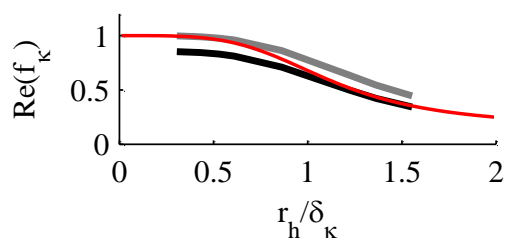

a)

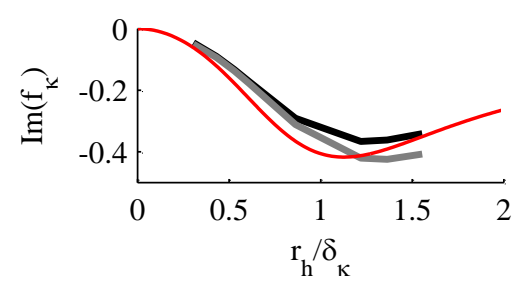

b)

Figure 5.44: a) real part and b) imaginary part of the thermal thermoacoustic functions of the fifth cylinder of the regenerator over the Lautrec number $r_{h} / \delta_{\kappa}$. The black line corresponds to the inline arrangement and the gray line to the alternating arrangement. The red line represents the thermoacoustic function for parallel plates as an indication.

a)
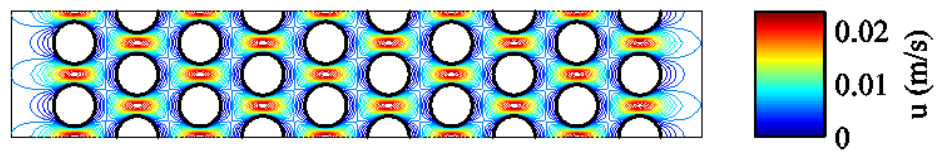

b)

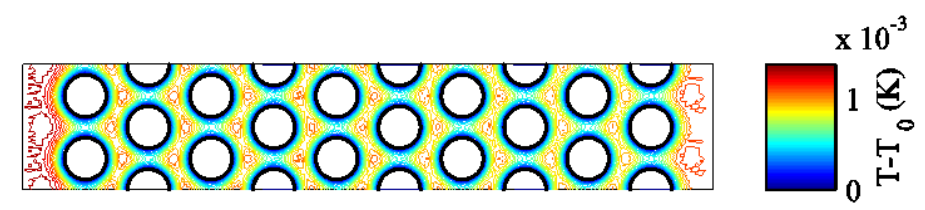

Figure 5.45: a) contour of the velocity and b) contour of the temperature at the moment of highest pressure and velocity, in the case of the simulation of $f=$ $1300 \mathrm{~Hz}$ and an alternating cylinder arrangement. Not the whole length of the computational domain is shown and the domain is repeated once along the periodic boundary condition.

\subsubsection{Influence of the drive ratio}

Up to now, the simulations were conducted with a pressure amplitude of $p_{1}=1 \mathrm{~Pa}$ which is very low. For this reason the pressure amplitude is increased here to $p_{1}=$ $100 \mathrm{~Pa}$, which is still a small amplitude for thermoacoustic applications but allows for a first estimation of the influence of the pressure amplitude.

The increase of the pressure amplitude does not have any effect on the viscous thermoacoustic function, as for both pressure amplitudes the viscous thermoacoustic functions overlay each other (not shown). The higher amplitude also does not have any effect on the thermal thermoacoustic function inside the regenerator (not shown), which means that the flow field does not affect the heat transfer inside the regenerator. This is an indi- 
cation that the differences in the thermal thermoacoustic functions for the two arrangements presented in the previous chapter are a result of the smaller distance between the gas and the solid and not to different flow fields.

The only deviation due to the pressure amplitude can be seen when the thermal thermoacoustic function is taken over the whole generator length. This is shown in Figure 5.46, where the thermal thermoacoustic function is taken over the whole generator for the two different amplitudes. In Figure $5.46 \mathrm{~b}$ ) the imaginary part of the thermal thermoacoustic function is given. The gray line represents the high amplitude while the black line represents the low amplitude. It can be seen that for a higher amplitude the deviation from the parallel plate behavior increases, which indicates that the entrance effects becomes more pronounced. This is especially true at low frequencies where the displacement amplitude is largest.

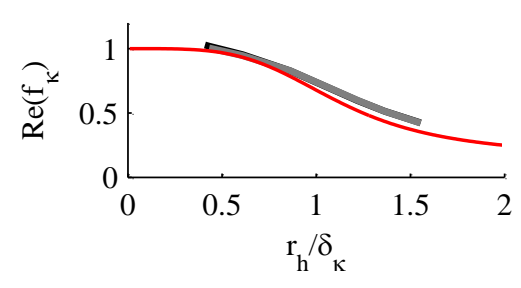

a)

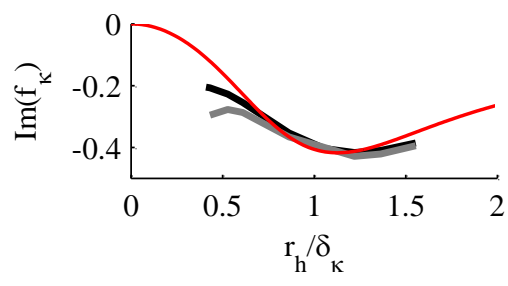

b)

Figure 5.46: a) real part and b) imaginary part of the thermal thermoacoustic functions of the fifth cylinder of the regenerator over the Lautrec number $r_{h} / \delta_{\kappa}$. The black line corresponds to a pressure amplitude of $p_{1}=1 \mathrm{~Pa}$ and the gray line to a pressure amplitude of $p_{1}=100 \mathrm{~Pa}$. The red line represents the thermoacoustic function for parallel plates as an indication.

\subsubsection{Conclusion and recommendations}

In this study the method of Udea et al. is successfully implemented for CFD and is applied to estimate the thermoacoustic functions of a simplified model of a stacked screen regenerator, which is modeled as inline cylinders. Using this method, it is demonstrated that the thermal and the viscous thermoacoustic function scale with a different length scale. The behavior of the thermoacoustic functions is explained by comparing with the thermoacoustic functions from the parallel plate geometry and a simplified model of the stacked screen regenerator. It is shown that the behavior of the thermoacoustic functions does not change when the whole numerical domain is scaled, indicating that the numerical domain can be scaled without loss of generality when no vortices or turbulence occur.

In a first step the number of cylinders, corresponding to the number of screens of the regenerator, is varied. Due to the entrance effects different thermal thermoacoustic functions are obtained, while the viscous ones are not affected by the entrance effects. In the simulated cases the entrance effects extend over two cylinders at both extremities of the 
regenerator. The thermoacoustic functions calculated over each cylinder located further inside the regenerator are the same independently of the length of the regenerator. This means that thermoacoustic functions calculated over one cylinder characterize a stacked screen regenerator without entrance effects.

By changing the arrangement of the screens it can be shown why porous materials and stacked screens are so widely used in thermoacoustic applications. Due to the different arrangement the largest distance between the gas and the solid is reduced, increasing the thermal contact while the viscous effects are not affected.

In the present study two different amplitudes are simulated, which can both be characterized as low for thermoacoustic applications. In this range the changes in pressure amplitude do not affect the thermoacoustic functions calculated over each cylinder inside the regenerator. Instead they only affect the entrance effects and thus the thermoacoustic functions calculated over the whole regenerator. As the entrance effects at the end of the regenerator play such an important role, they are investigated in more details in the following chapter.

In future studies different geometric influence factors might be investigated. For example the opening could be varied, as this would allow obtaining the optimal opening, which is expected to be larger than the one of the present numerical model. In addition to this, the model can be extended to the third dimension, allowing for more realistic models of the stacked screen regenerator. With this model it would be possible to investigate different packing of the screens.

The present model can be extended in future work for even higher amplitudes where vortices at the extremities of the regenerator could possibly occur. These vortices play an important role in the accurate estimation of the thermoacoustic functions at high amplitude. In order to accurately predict the thermoacoustic functions for higher amplitudes, the length of the regenerator model has to be increased, such that the entrance effects do not influence the thermoacoustic functions calculated over a cylinder inside the regenerator. Besides this the model presented here would allow investigating the influence of vortex generation on heat transfer at the ends of the regenerator in the future. 


\subsection{Entrance effects in stacked screen regenerator ${ }^{1}$}

The regenerator is the key component of a traveling wave thermoacoustic device in which the thermoacoustic effect occurs. In traveling wave devices the thermoacoustic effect occurs within one penetration depth, as in an ideal case the local gas temperature has to follow the solid temperature of the regenerator (see Chapter 2.1.2.2). Gas parcels further away than one penetration depth do not interact with the solid and therefore do not undergo the thermoacoustic cycle. For high power densities the volume not contributing to the energy conversion has to be minimized. However, if the pores are too small, viscous losses become dominant and can lead to low efficiencies. Hence, both the thermal and viscous effects have to be balanced in the thermoacoustic regenerator.

Furthermore, heat conduction in the wave propagation direction is an unwanted effect and can be considered as a loss. One way of minimizing this effect, is to use stacked screens as they have only tiny contact points in the wave propagation direction. The vast mesh sizes readily available allow easy adjustment of the open area in the wave propagation direction for optimal balancing of the thermal and viscous effects [117].

In this chapter the entrance effects at both ends of stacked screen regenerators are investigated. These effects are not accurately predicted by the one-dimensional thermoacoustic equations as the assumption that the displacement amplitude $\xi_{1}$ is much smaller than all other dimensions in the $x$-direction is violated. At the interface between the regenerator and the thermal buffer tube the convective effects $(u \cdot \nabla) T$ become important and lead to a change in mean temperature which can be on the order of the adiabatic temperature oscillation [78]. This nonlinear effect can lead to an increase in thermal losses, as a steeper temperature gradient occurs in the thermal buffer tube [92].

The change in mean temperature was qualitatively explained by Swift [6] and Kittel et al. [118] by following gas parcels which start within two displacements amplitudes of the entrance of a heat exchanger. Summing at one fixed position the temperature of the gas parcels passing this point, the mean temperature profile close to the entrance of the heat exchanger can be obtained. This leads to a joining condition in the mean temperature which is widely accepted and implemented in one-dimensional codes like DeltaEC [7] and Sage [48].

Analytical solutions were derived by Matveev et al. [78] and Gusev et al. [77] for the simplified case in which both the heat conduction in the wave propagation direction as well as the viscous effects are neglected.

Next to the analytical solution, also numerical models that include heat conduction in the wave propagation direction were presented by Matveev et al. [76] and Berson et

\footnotetext{
${ }^{1}$ Parts of this chapter are published in proceedings of the IHTC-15 and PAMIR conference.
} 
al. [92]. These results are compared to experimental results in the respective papers and showed good agreement for traveling wave phasing.

In this work the interaction of the working fluid with a regenerator is investigated with CFD simulations. Simulating the whole three-dimensional flow field would be computationally too expensive, for this reason a reduced CFD model is presented. As the entrance effects shall be investigated, the regenerator is reduced to two meshed screens. Assuming that the screens are perfectly inline, and neglecting gravity effects and the effects of the duct where the regenerator is housed, the whole mesh can be modeled as a periodic repetition of one cell of the meshed screen. In a first approach this cell is further reduced to two inline cylinders, where each cylinder represents the wire of a screen. The geometric reduction is summarized in Figure 5.47.

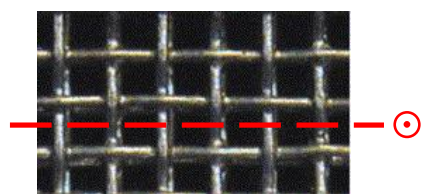

a)

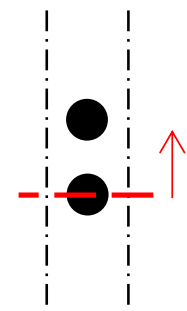

b)

Figure 5.47: Visualization of geometric reduction. a) full screen; b) reduced CFD model. Red arrow indicates the wave propagation direction.

Most of the published work on flow around cylinders focuses on the hydrodynamic forces and vortex generation in a steady flow, for application to offshore platforms. Extensive experimental work on steady flow around a cylinder and pairs of cylinders has been carried out, for example by Williamson [119]. Next to experiments also numerical simulations were conducted in order to reveal the flow field. An et al. [120] used a finite element code to determine the force acting on two cylinders in tandem. Anagnostopoulos et al. did the same for four cylinders in a square arrangement [121] as well as a pair of cylinders in different configurations [122, 123].

Heat transfer on one cylinder in pure oscillating flow was also investigated with simulations and experiments. Mozurkewich [124] investigated the heat transfer around a single cylinder in a standing wave experimentally. Gopinath et al. [125] carried out experiments for low amplitude oscillatory flow, for which the displacement amplitude is small compared to the cylinder diameter. Iwai et al. [126] used numerical simulations and experiments to investigate the heat transfer from one cylinder for displacement amplitudes much higher than the cylinder diameter.

In this study two inline cylinders in the oscillating flow direction and repeated in the direction orthogonal to the flow are simulated to estimate the heat transfer and the en- 
trance effects of a stacked screen. The displacement amplitude is about eleven times the cylinder diameter. This corresponds to a Keulegan-Carpenter number of $K C=$ $u_{1} /(f D) \approx 69$.

\subsubsection{Entrance effect models}

In this chapter the models of the stacked screen regenerator are presented in order to characterize the entrance effects. The reduced model consists of two cylinders in an inline arrangement in the wave propagation direction and repeated in the direction orthogonal to the wave propagation direction.

In chapter 5.4.1.1 the CFD model with boundary conditions is presented and the onedimensional model to which the CFD simulations are compared in chapter 5.4.1.2.

\subsubsection{CFD model}

The regenerator is reduced to two inline cylinders as described above. These cylinders model the wires of the stacked screen regenerator. In this chapter only the characteristics of the reference case are presented, from which the other end effect CFD models are derived. The reference model with its boundary conditions is shown schematically in Figure 5.48.

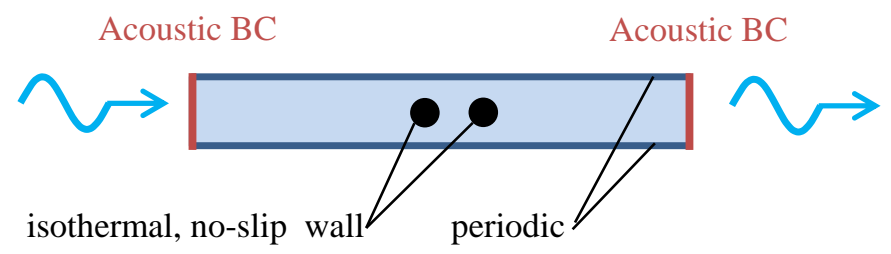

Figure 5.48: Boundary conditions of the stacked screen heat exchanger these case.

The boundary conditions at the left and right side of the domain are non-reflecting boundary conditions as they were presented in Chapter 3.2.1.2. Using these it is possible to impose a traveling wave that enters the domain at the left and exits the domain at the right without reflections. The temperature of the fluid entering the domain is calculated by assuming an adiabatic process. Due to this assumption the distance between the screen and the acoustic boundaries has to be large enough such that the wave is close to adiabatic at the boundary. The entrance length $L_{1}$ is estimated as:

$$
L_{1}=\left(\delta_{\kappa}+2 \xi_{1}\right) S
$$

where $S$ is a safety factor. The safety factor is introduced as the interaction of the wave with the cylinders introduces a mean temperature difference as was shown for the ideal heat exchanger in Chapter 4.5. Due to the temperature differences, heat is conducted towards the boundaries. After some periods a heat flux over the boundary will take place. This can be seen by a small temperature jump at the moment when the flow direction reverses. It could be shown that this does not have an effect on the heat transfer on the 
cylinders when the boundary conditions are far enough from the cylinders, that is for $S=1.5$.

The frequency of the incoming wave is set to $f=100 \mathrm{~Hz}$. The mean pressure and temperature are chosen to be $p_{0}=1 \mathrm{~atm}$ and $T_{0}=300 \mathrm{~K}$. The working gas used in the simulations is helium (see Appendix E), as it is frequently used in thermoacoustic cryocoolers because of its low condensation temperature and its favorable heat transfer characteristics [6].

The dimensions of the model are shown in Figure 5.49. The half spacing $h$ corresponding to half of the opening of the screen is chosen such that it is one thermal penetration depths $\delta_{\kappa}$ large. Due to the high heat capacity of the metal wire compared to the working gas at ambient mean pressure and low pressure amplitude, the wire diameter is not defined from a thermal point of view, but rather a production point of view. For this reason the mesh was chosen from available screens with the required opening and the smallest wire diameter $D=0.00022 \mathrm{~m}$. The distance in wave propagation direction between the two wires $L_{2}$ is two diameters $D$.

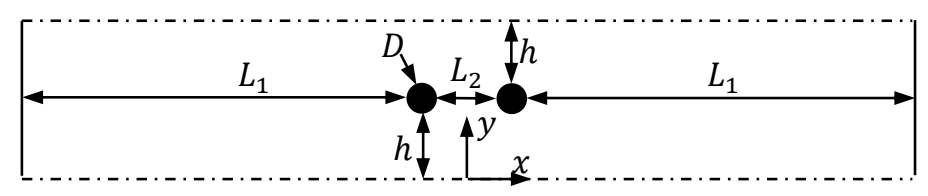

Figure 5.49: Definition of the dimensions of the entrance effect CFD model.

The pressure amplitude of the incoming wave is set to $p_{1}=250 \mathrm{~Pa}$. This corresponds to a drive ratio of $D r=0.25 \%$ which is low for a thermoacoustic application, but still allows the estimation of deviations from the thermoacoustic equations. All simulations are carried out for five wave periods, which represents a total simulation time of $t=$ $0.05 \mathrm{~s}$. Figure 5.50 shows the heat flux averaged over one period from the left cylinder to the fluid for the four last periods. As the changes in time are very small, it can be concluded that five periods are enough to reach a steady periodic state.

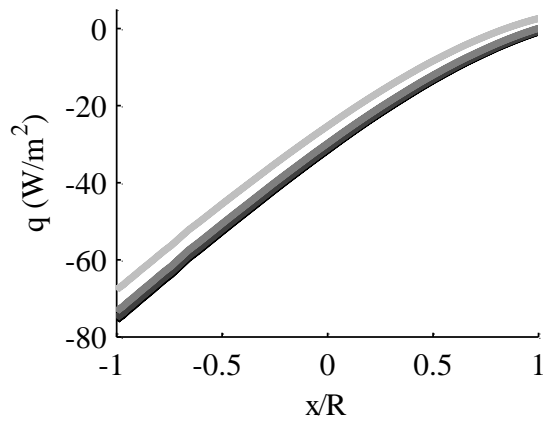

Figure 5.50: Heat flux over one period from the left cylinder to the fluid for the last four periods. Increasing number of the period corresponds to darker line. 
In order to resolve the flow field inside the model defined above, the domain has to be discretized. Figure 5.51 shows the computational mesh of the reference model. While the total domain height is shown, the domain extends further in the $x$-direction. An O-grid mesh is placed around each cylinder, with 120 grid points over the perimeter and 32 grid points in the radial direction, geometrically growing with a factor of 1.2 away from the cylinder. The rest of the mesh consists of rectangular elements with a constant height. At the left and right of the O-mesh, the cells grow geometrically with a factor of 1.2 towards the acoustic boundaries. This is possible as the gradients in the $x$-direction decrease with increasing distance to the cylinders. This is a consequence of the nearly adiabatic wave propagation occurring away from the cylinders and of the small domain size compared to the wave length. This has two positive effects: fewer elements are needed in the $x$ direction and due to the large element size $\Delta x$ at the boundary, the time step size can be increased. In the reference case this leads to a time step size of $\Delta t=1.5 \cdot 10^{-6} \mathrm{~s}$ which corresponds to more than 6600 time steps per period. The total domain is discretized with 23673 nodes.

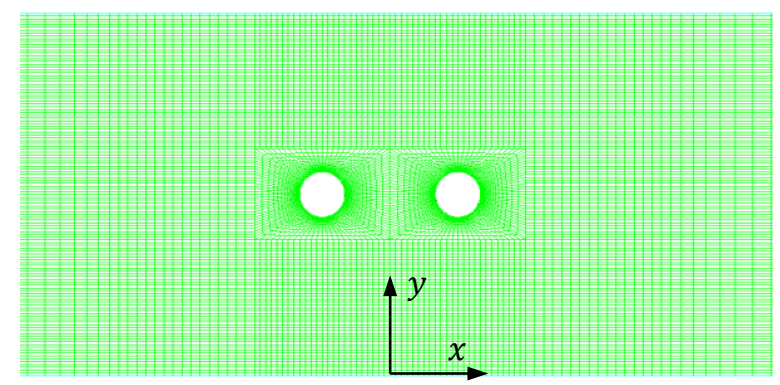

Figure 5.51: Detail of the mesh of the reference model. While the total domain height is shown, the domain extends further in the $x$-direction.

The mesh has been validated in two steps (not shown here). In a first step a mesh dependency study was carried out. The number of elements in each direction was doubled and the geometric growing factors set to 1.1. The results were compared with the reference mesh in terms of the heat transfer parameters presented in the next chapter. In a second step the entrance length was increased. In both cases the changes on the heat transfer parameters stayed below $1 \%$. It can thus be concluded that the mesh is sufficiently fine to investigate the heat transfer characteristics of staggered screens with this model.

\subsubsection{One-dimensional model}

The CFD model is compared to a one-dimensional model which is similar to the one used for the validation of the thermal buffer tube simulations given in Chapter 5.1.1.2. The one-dimensional heat equation (Equation (5.5)) is solved, assuming that the pressure and velocity are known. As the domain length is small compared to the wave length, the pressure and the volume flow rate are assumed uniform in the whole domain. The chang- 
es in velocity due to the narrowing of the fluid domain at the cylinders are taken into account in the simulations.

The model has the same axial lengths as the CFD model (shown in Figure 5.49) and the cylinders are modeled by setting the heat transfer coefficient $K \neq 0$ over the length of the two cylinders and zero everywhere else. The heat transfer coefficient $K$ is calculated by approximating the cylinder as two parallel plates with a half spacing corresponding to the hydraulic radius $r_{h}$ of the cylinders. The geometrical reduction for the estimation of the hydraulic radius is visualized in Figure 5.52. Figure 5.52 a) shows the geometry of the CFD simulation and Figure 5.52 b) shows the corresponding parallel plate reduced geometry. The necessary hydraulic radius for the parallel plate model can be calculated as follows:

$$
r_{h}=\frac{D \cdot(2 h+D)-\pi\left(\frac{D}{2}\right)^{2}}{\pi D}
$$

The thermal thermoacoustic function of parallel plates can be calculated from the hydraulic radius and the corresponding thermal penetration depth according to Equation (B.2). This allows the estimation of the heat transfer coefficient in the case in which no wall temperature gradient occurs [93]:

$$
K=\left|i \omega \frac{f_{\kappa}}{1-f_{\kappa}}\right|=2100 \frac{1}{\mathrm{~s}}
$$

The domain is initialized with a constant temperature of $T_{0}=300 \mathrm{~K}$. The temperature boundary conditions at both ends are calculated from the pressure assuming an adiabatic process at the boundary, similar to what is done in the CFD model.

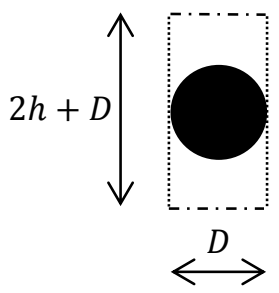

a)

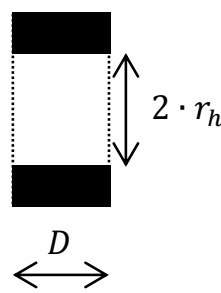

b)

Figure 5.52: a) cylinder modeling the stacked screens and b) the corresponding simplified parallel plate geometry with the definition of the corresponding dimension.

\subsubsection{Results and discussion}

The results of the CFD model are discussed in this chapter. In a first step the results of the reference case are given. In a second step the results for different numbers of cylinders, different openings of the screen and a different wave phasing are addressed in Chapter 5.4.2.1, 5.4.2.2 and 5.4.2.3 respectively. 
The time dependent flow field of the reference case is first presented in this section. The incoming wave travels through the domain and interacts with the two cylinders. Figure 5.53 (a) shows the velocity in the wave propagation direction over the $x$ coordinate. The velocity is normalized by the velocity amplitude at the left boundary and is plotted at four different instances in time and at two different $y$-locations. The first $y$ location is on the periodic boundary condition $(y=0$, black line), the second goes through the center of the cylinder $(y=h+D / 2$, gray line). The $x$-coordinate is normalized with the displacement amplitude $\xi_{1}$. At the boundaries there is no difference between the $x$-velocity at the two different heights. It can be concluded that the flow field is onedimensional close to the acoustic boundaries.
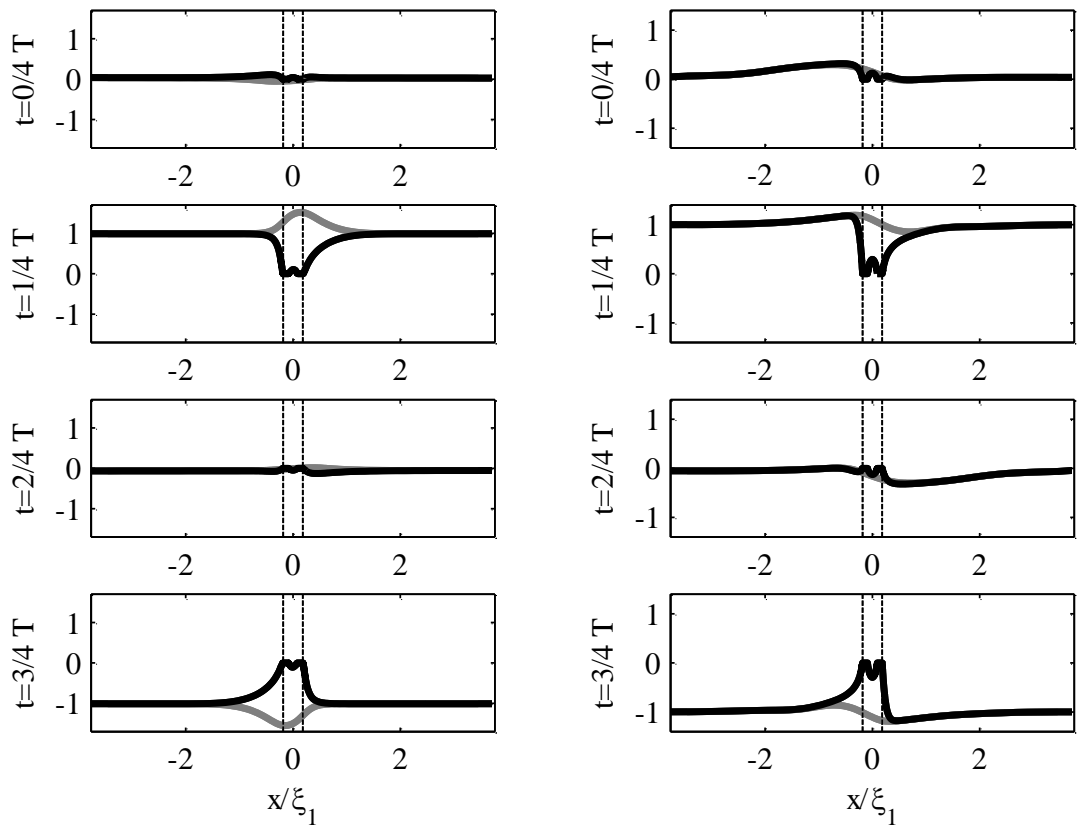

a) velocity

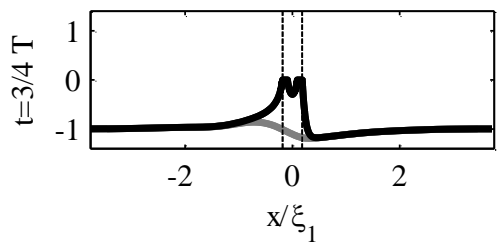

b) temperature

Figure 5.53: a) Velocity in the wave propagation direction and b) temperature, normalized with the respective amplitude at the left extremity, at four different instances in time separated by a quarter period each and at two different heights (black line $y=0$ and gray line $y=h+D / 2$ ) over the $x$-coordinate normalized with the displacement amplitude $\xi_{1}$. The vertical dashed lines indicate the extremities of the stacked screens.

In the vicinity of the cylinders the flow field is two-dimensional. Due to convection, a compression area develops in front of the cylinder and a wake area develops behind the cylinder. This is visible in the velocity profile at the instance in time $t=1 / 4 \cdot T$. The maximum velocity is not at $x / \xi_{1}=0$, but convected to higher $x / \xi_{1}$-values. Further- 
more, the velocity profiles are not symmetric around the cylinders. In an acoustic code this would not be taken into account and the maximum velocity would occur between the two cylinders at $x / \xi_{1}=0$. In Figure $5.53 \mathrm{~b}$ ) the normalized temperature oscillation over the $x$-coordinate is shown at four different time instances of a period. It can be seen that heat is supplied to the left cylinder, as at the instance in time $t=0 \cdot T$ the temperature for negative $x / \xi_{1}$-values is higher than the adiabatic temperature and will increase even further during the following quarter cycle due to compression. At the same time, the fluid is convected to the regenerator material and the left screen heats up. During the half cycle with a negative velocity, the fluid temperature for positive $x / \xi_{1}$-values is lower than the cylinder temperature: due to the convection the right cylinder is cooled. Over a complete cycle the left cylinder will heat up and in the same manner the right cylinder is cooled.

The underlying heat pumping cycle can be revealed by following a gas parcel which starts at $x=0$ and $y=h / 2$ over several periods. The numerical approach using the Runge-Kutta method to track the position of the gas parcel from the CFD simulation data is presented in Appendix D. The obtained trajectory of the gas parcel is shown in Figure 5.54 a) in black. The cross indicates the starting position. The trajectory of the gas parcel forms a small hysteresis, indicating that the flow is not symmetric. The asymmetry is due to the compression and wake areas around the cylinders which lead to a different trajectory depending on the flow direction.

Knowing the state of the gas parcel at the different positions, the $p-v$-diagram shown in black in Figure 5.54 b) can be obtained. An area is enclosed and the resulting cycle is in counterclockwise direction, indicating heat pumping. The cycle that the gas parcel undergoes is shown schematically in Figure $5.54 \mathrm{c}$ ) and can be summarized by the following four steps:
(A-B) adiabatic compression
(B-C) isobaric cooling
(C-D) adiabatic expansion
(D-A) isobaric heating.

These four steps correspond to the ideal Brayton cycle [127]. Due to the heat pumped an additional thermal load is added to the regenerator.

In typical cryocoolers operating at high amplitudes, the displacement amplitude is on the order of one tenth of the regenerator length [6], thus the cycle described above cannot be performed entirely. One adiabatic stage cannot be performed, for example stage (A-B) for a parcel entering the regenerator at the right. This stage will be an isothermal compression instead. However, heat pumping still occurs. To demonstrate this, a gas parcel is chosen that does not entirely pass the two cylinders. The starting position of this parcel is $x=\xi_{1}$ and $y=h / 2$. The position of this gas parcel and the corresponding $p$ - $v$ diagram are shown as gray lines in Figure 5.54 a) and b) respectively. There are no isobaric stages (B-C, D-A), which means that the cooling and heating processes are no longer isobaric. 
Due to this, the enclosed area is smaller, but still heat is pumped at the extremity of the regenerator due to convective effects.

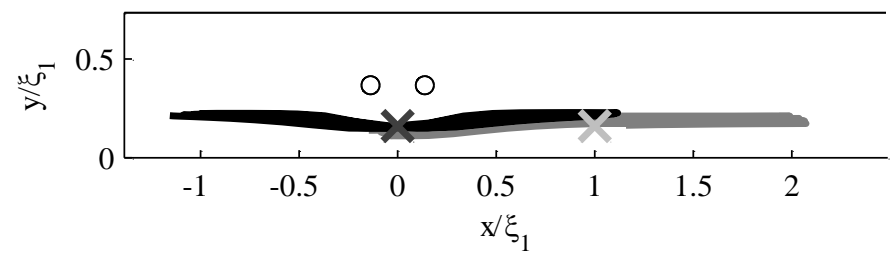

a)

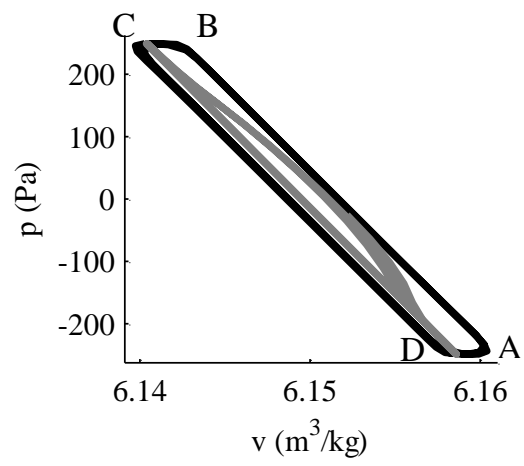

b)

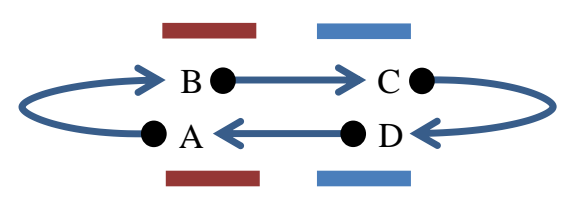

c)

Figure 5.54: a) Position during the last 4 periods of the gas parcels starting at the following positions (crosses): black $x=0, y=h / 2$ and gray $x=\xi_{1}$, $y=h / 2$. b) $p-v$ diagram of corresponding fluid parcels. c) Schematic of the thermodynamic cycle of the gas parcel: (A-B) adiabatic compression (B-C) isobaric cooling (C-D) adiabatic expansion (D-A) isobaric heating.

Another effect which is not resolved by the one-dimensional codes is the buildup of mean temperature around the cylinder, which is also due to the convective effects at the interface between the regenerator and the open space. The change in mean temperature can be seen in Figure 5.53 b). At every time instance the gas at the left proximity of the cylinders has a higher temperature than further away, where the gas is subject to adiabatic temperature changes.

Figure 5.55 shows the changes in mean temperature normalized with the temperature amplitude based on adiabatic wave propagation. The mean temperature is calculated by averaging the temperature over the last period. At the left of the cylinders, the mean temperature increases, while at the right the mean temperature decreases. The maximum and the minimum mean temperature can be found at the height $y=h+D / 2$ within one displacement amplitude of the cylinders. In the reference case the change in mean temperature corresponds to $20 \%$ of the temperature oscillation. Especially within one displacement amplitude from the cylinder, the flow field shows a two-dimensional character. The 
changes in mean temperature are largest in this area and introduce a thermal load on the regenerator.
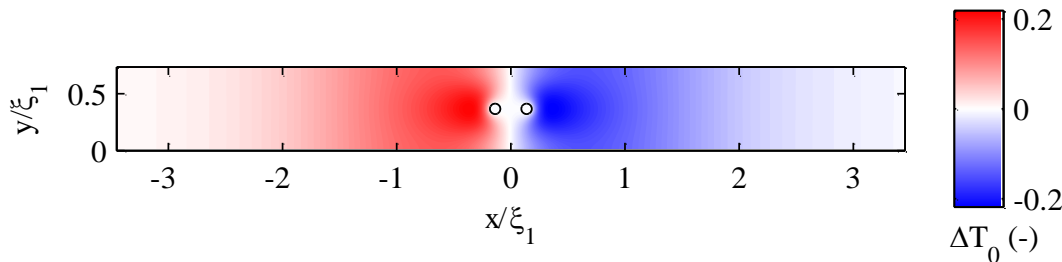

Figure 5.55: Changes in mean temperature averaged over the last period and normalized with the adiabatic temperature amplitude. The mean temperature is taken over the fifth period.

The CFD simulations can be compared with the one-dimensional model presented in Chapter 5.4.1.2. The gray solid line in Figure 5.56 shows the changes in mean temperature of the CFD simulation. As the temperature field is two-dimensional, the changes in mean temperature from the simulations are area averaged over the height in all figures in this chapter. The dotted black line shows the changes in mean temperature obtained from the one-dimensional model. The changes in mean temperature are underestimated in the one-dimensional model, in the region where the maximum occurs. The reason for this deviation will be investigated in the following chapters and the influence on the entrance effects of the regenerator length, the mesh opening and the wave phasing are presented.

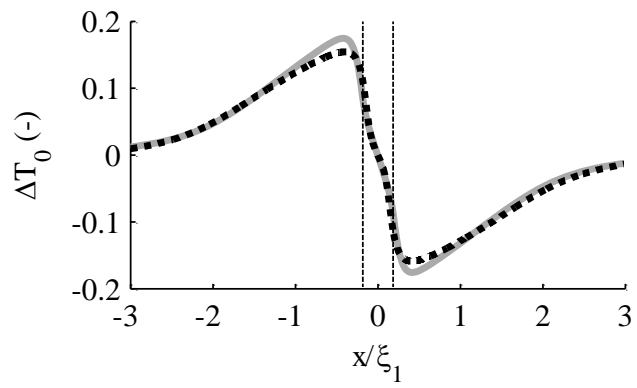

Figure 5.56: Changes in mean temperature normalized by the adiabatic temperature amplitude, plotted over the dimensionless axial-coordinate $x / \xi_{1}$. The mean of the temperature is taken over the fifth period. The dotted black lines show the results from the one-dimensional model, while the gray solid line shows the results of the CFD simulation. The temperature data from the simulation is surface average over the height of the domain. The vertical dashed lines indicate the extremities of the stacked screens. 


\subsubsection{Influence of the regenerator length}

In this chapter the influence of the regenerator length is investigated. Four simulations modeling the regenerator with one, two, three and five cylinders are compared. A further increase in the number of cylinders is investigated with the one-dimensional model only. Figure 5.57 shows the changes in mean temperature over the dimensionless axialcoordinate $x / \xi_{1}$ for one two, three and five cylinders. The solid lines show the results from the simulations, while the dashed lines show the results from the one-dimensional model. With an increasing number of cylinders the changes in mean temperature increase in both models. This is due to the entrance effects at the extremities of the regenerator model. With an increasing number of cylinders, the gas parcel is in thermal contact with the regenerator over a larger part of its displacement amplitude. Because of this, the gas parcels leaving the regenerator have a temperature closer to the temperature of the regenerator material. The following adiabatic compression that the gas parcel undergoes outside of the regenerator, leads to a larger change of the mean temperature. This is the same effect as was described for an ideal heat exchanger in Chapter 4.5.

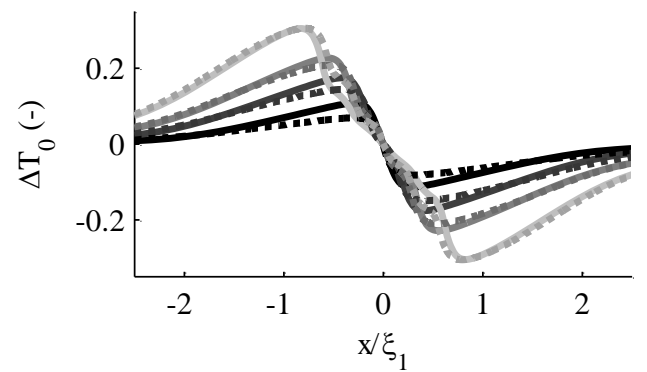

Figure 5.57: Changes in mean temperature normalized by the adiabatic temperature amplitude, plotted over the dimensionless axial-coordinate $x / \xi_{1}$. The mean of the temperature is taken over the fifth period. Results from the CFD simulation for one, two, three and five cylinders are shown with solid lines. The corresponding one-dimensional model results are shown with the dotted lines. Lines become lighter with an increasing number of cylinders. The temperature data from the simulation is area averaged over the height of the domain.

Comparing the CFD simulations with the one-dimensional models, it can be seen that they agree qualitatively, as they show the same profile with an increase in mean temperature at the left of the cylinder and a decrease at the right of the cylinder. Still small quantitative differences occur. For a low number of cylinders the changes in mean temperature are under predicted by the model, while the model over predicts the changes in mean temperature for a higher number of cylinders. The reason for this is that the onedimensional model overestimates the heat transfer at the cylinders, resulting in a larger change in mean temperature over the length of the cylinder. On the other side, the onedimensional model underestimates the heat transfer just outside of the cylinders, as the 
heat transfer at this location is due to two-dimensional effects: the one-dimensional model only takes into account the heat transfer orthogonal to the wave propagation and not the heat transferred from the cylinder in horizontal direction. These two effects can explain why the changes in mean temperature are under- or overestimated by the onedimensional model. For a low number of cylinders the changes in mean temperature are underestimated, as the heat transfer just outside of the cylinders has a larger relative influence. With an increasing number of cylinders, the overestimated heat transfer at the cylinders becomes more and more important, the one-dimensional model overestimates the changes in mean temperature. In engine applications the number of cylinders would be considerably higher, meaning that the one-dimensional model would overestimate the changes in mean temperature when a real regenerator is modeled like it is proposed in this thesis.

But for an even higher number of cylinders, when the length of the regenerator exceeds two displacement amplitudes, additional cylinders do not have an influence on the changes in mean temperature, as the additional cylinder at the inside of the regenerator will only be seen by gas parcels that are not leaving the regenerator. Figure 5.58 shows the changes in mean temperature obtained from the one-dimensional model for an increasing number of cylinders. The profile is qualitatively the same for all numbers of cylinders, meaning that for a traveling wave propagating from left to right the mean temperature increases at the left of the regenerator and decreases at the right of the regenerator. The maximum changes of the mean temperature are indicated by the red line in Figure 5.58 and converge to a constant value for a larger numbers of cylinders. The reason for this is that the entrance effects are due to the interaction of gas parcels with both the regenerator and the open space. For short regenerators, the thermal contact between the fluid and the regenerator is loose as the gas parcels pass the entire regenerator. The changes in mean temperature are small. When the regenerator becomes longer, the thermal contact becomes closer and the changes in mean temperature increase due to the earlier mentioned thermodynamic cycle. When the regenerator becomes longer than the total displacement of the gas parcels, a further increase in length no longer changes the entrance effects. This means that the maximum changes of the mean temperature converge to a constant value.

The displacement amplitude calculated from the velocity outside of the regenerator corresponds to roughly seven cylinders. This is in accordance with the results from the one-dimensional model, for which the maximum change in mean temperature converges from roughly nine cylinders on. The slightly higher number could be due to the higher velocity at the cylinders, due to the smaller opening. A higher velocity leads to an increased displacement amplitude.

From the one-dimensional model, it can be concluded, that for an increasing number of cylinders the differences between CFD results and the one-dimensional model should become constant. The reason is that from a regenerator length of two displacement ampli- 
tudes on, additional cylinders do not have any further effect on the changes in mean temperature. It can be concluded that the one-dimensional model predicts the changes in mean temperature qualitatively correctly, but overestimates them slightly for long regenerators.

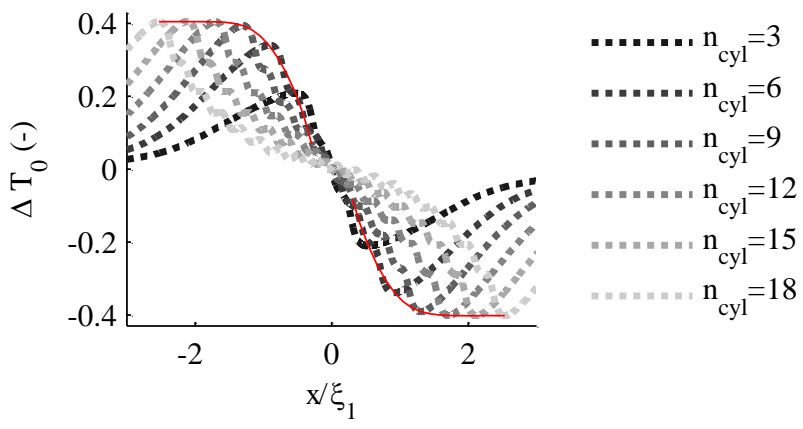

Figure 5.58: Changes in mean temperature obtained from the one-dimensional model for different numbers of cylinders, normalized by the adiabatic temperature amplitude and plotted over the dimensionless axial-coordinate $x / \xi_{1}$. The mean of the temperature is taken over the fifth period. Red line indicates the location of the maximum and minimum values of the temperature changes.

For regenerators larger than two displacement amplitudes the maximum change in mean temperature is only dependent on the heat transfer coefficient $K$ and thus the opening of the regenerator. For this reason the next parameter investigated is the opening of the screens.

\subsubsection{Influence of different openings of the meshed screen}

In this chapter the influence of the opening (the mesh size) on the entrance effects is investigated. Figure 5.59 shows the changes in mean temperature for three different openings. The changes in mean temperature are normalized with the adiabatic temperature amplitude and plotted over the dimensionless axial-coordinate $x / \xi_{1}$. The black lines, the dark gray lines and the light gray lines show the results for the different openings $h$, corresponding to approximately one third, one and two times the thermal penetration depth respectively. The dark gray line corresponds to the reference case defined in Chapter 5.4.1.1. With an increasing opening, parts of the fluid passing the stacked screens do not thermally interact with the screens. For the largest opening, the fluid at a height of $y=0$ is outside the thermal boundary layer and does not interact with the stacked screens. This can be shown by following a gas parcel that starts at $x=0$ and $y=0$. The corresponding $p$ - $v$-diagram for the three different openings is shown in Figure 5.60. It can clearly be seen that for the largest opening (light gray line) no heat is pumped, as no surface is enclosed in the $p$ - $v$-diagram. For the smaller openings (black line) the gas-solid thermal 
contact increases and more heat is exchanged between the fluid and the cylinder. The surface enclosed in the $p$-v-diagram becomes larger. Due to this, the changes in mean temperature increase with decreasing opening and will converge in the limit of a perfect heat exchanger. The maximum dimensionless temperature change can be estimated from the ideal heat exchange model of Matveev et al. [76] presented in Chapter 4.5.1.1, resulting in a dimensionless temperature change of $\Delta T_{0}=2 / \pi \approx 0.64$.

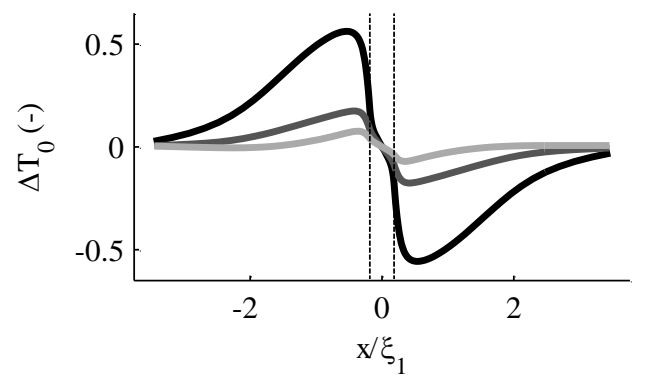

Figure 5.59: Changes in mean temperature obtained from CFD, normalized by the adiabatic temperature amplitude and plotted over the dimensionless axialcoordinate $x / \xi_{1}$ in the case of the stacked screen model. The mean of the temperature is taken over the fifth period and area averaged over the height of the domain. The black lines, the dark gray lines and the light gray lines show the results for the mesh openings $h=D, h=3.5 \cdot R$ and $h=$ $7.5 \cdot R$ respectively. The three heights correspond roughly to an opening of one third, one and two thermal penetration depths respectively. Vertical dashed lines indicate the extremities of the stacked screens.

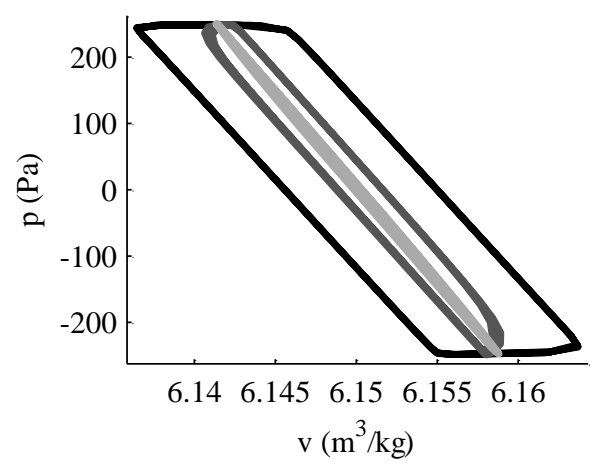

Figure 5.60: $\quad p$-v-diagram for the fluid parcels starting at position $x=0, y=0$ in the stacked screen model. The black lines, the dark gray line and the light gray line show the results for the mesh openings $h=D, h=3.5 \cdot R$ and $h=7.5 \cdot R$ respectively. The three heights correspond roughly to an opening of one third, one and two thermal penetration depths respectively. 
Not only the mean temperature changes when the thermal contact is varied, but also the heat pumped from one cylinder to the other. To estimate the total heat transferred, each screen is assumed to be square in shape with side lengths of $L_{y}=L_{z}=5 \mathrm{~cm}$, which would be a realistic size for thermoacoustic applications. This means that the cylinders in the simulation represent wires with a length of $L_{z}$. The heat transfer from one of these wires is investigated in the following. Figure 5.61 shows the heat transferred from the right cylinder to the fluid over the dimensionless half spacing $h / \delta_{\kappa}$. With larger openings the heat transferred decreases. This is due to the earlier described heat pumping cycle which needs close thermal contact in order to exchange heat in the isobaric stage. This is especially true for short stacked screen regenerators like in the CFD simulations, where the total regenerator length is only a fraction of the displacement amplitude. For increasing openings, especially $h>\delta_{\kappa}$ the heat transferred from the cylinder converges to a constant value. The reason for this is that the fluid further away than one thermal penetration depth, does not thermally interact with the cylinder.

But the interaction of the acoustic wave with the regenerator also introduces acoustic power losses. Next to the viscous and thermal effect the heat pumping cycle dissipates acoustic power. The total acoustic power dissipated is shown in Figure $5.61 \mathrm{~b}$ ) as a function of the opening. The acoustic power dissipation has a similar behavior as the heat transferred. With increasing opening, the acoustic power dissipation converges to a constant value, as the fluid outside of the penetration depth does not interact with the solid and propagates without losses. For smaller openings the acoustic power dissipation increases due to the increased viscous and thermal losses.

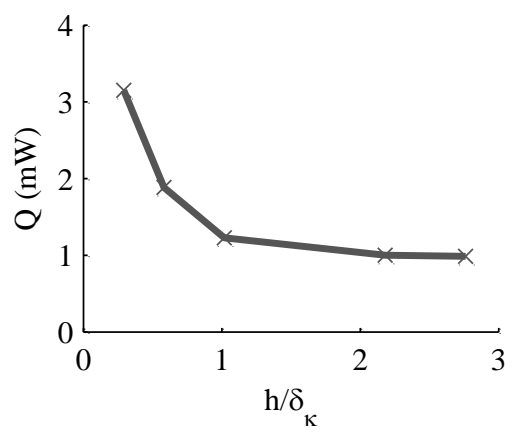

a)

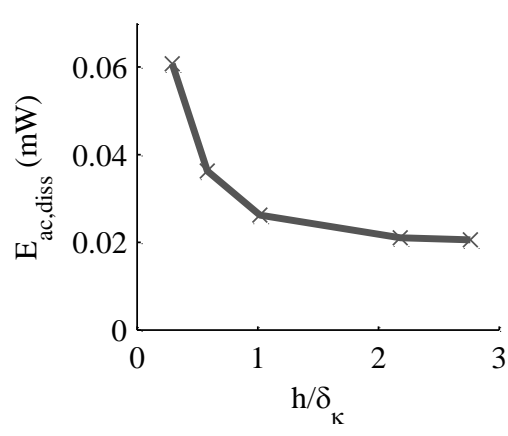

b)

Figure 5.61: a) Heat transferred from the right cylinder to the fluid and b) dissipated acoustic power over the dimensionless half spacing $h / \delta_{\kappa}$.

In the previous chapter it was shown that the length of the regenerator plays an important role, especially for short regenerators. The qualitative behavior for a larger number of cylinders (not shown) is the same as the one shown in Figure 5.61. The heat transferred and the acoustic power dissipated decrease with increasing opening and converge to a constant value. These values are larger for a higher number of cylinders. 


\subsubsection{Influence of the wave phasing}

In the previous sections the geometrical influence was investigated, but the phasing of the wave also plays an important role on the entrance effects. In this section the entrance effects for traveling wave phasing are compared with the entrance effects for standing wave phasing. Matveev et al. [76] reported that the one-dimensional model shows deviations from experiments for standing waves phasing, this will be investigated using CFD.

In the CFD simulation the standing wave phasing is achieved by superposition of two waves with the same amplitude. This means that an additional wave is introduced at the right acoustic boundary. In order to make the simulations comparable with the traveling wave simulations, the position within the standing wave is chosen such that the pressure and the velocity amplitude are the same as in the traveling wave case. As the domain is short compared to the wave length, the phase difference between the two introduced waves can be chosen with a delay of $\varphi=\pi / 2$. Furthermore, due to the superposition of the two waves, the amplitude of each wave has to be divided by $\sqrt{2}$. This leads to a standing wave where the velocity and pressure amplitudes are the same as for the traveling wave case, but the velocity and pressure are $\pi / 2$ out of phase.

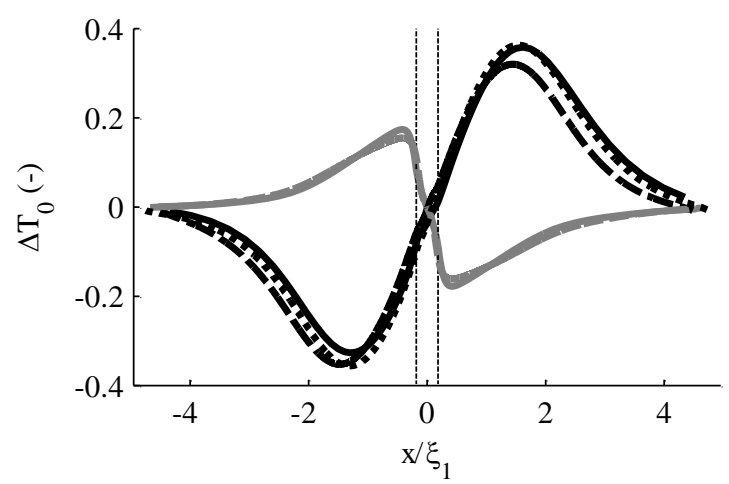

Figure 5.62: Changes in mean temperature normalized by the adiabatic temperature amplitude and plotted over the dimensionless axial-coordinate $x / \xi_{1}$ for the two different wave phases. The mean of the temperature is taken over the fifth period and area averaged over the height of the domain in the case of the CFD simulations. Results for standing wave and traveling wave are shown with black and gray lines, respectively. Solid lines show results from CFD, while dashed lines show the results from the one-dimensional model. The dotted black line shows the results from the one-dimensional model when streaming is incorporated. The vertical dashed lines indicate the extremities of the stacked screens.

Figure 5.62 shows the changes in mean temperature normalized by the adiabatic temperature amplitude, plotted over the dimensionless axial-coordinate $x / \xi_{1}$ for the two different phases. The gray lines show the traveling wave phasing results, while the black lines show the standing wave results. The solid lines represent the results from CFD and 
are first compared with each other. The profiles are clearly different for both phases, as the increase in mean temperature is situated at the right of the regenerator for the standing wave case and at the left for the traveling wave case. Another difference is that the amplitude of the temperature overshoot in the case of a standing wave is higher than for the traveling wave. This is not generally the case, as can be shown with the one-dimensional model. The maximum changes in mean temperature are plotted over the normalized hydraulic radius in Figure 5.63 for standing waves (black line) and traveling waves (gray line). The solid lines represent the results for two cylinders as used in the reference geometry. The red vertical line indicates the hydraulic radius $r_{h} / \delta_{\kappa}=0.67$ corresponding to the reference geometry. As was shown from the simulations and the one-dimensional model in Figure 5.62, the changes in mean temperature are larger for the standing wave case.

The dashed lines show the results from the one-dimensional model with ten cylinders, which is long enough that additional cylinders no longer affect the entrance effects. For the standing wave phasing the maximum value in Figure 5.63 is shifted towards $r_{h} / \delta_{\kappa} \approx$ 0.7. The maximum occurs due to the thermoacoustic effect that occurs inside of the regenerator and which induces a temperature gradient and due to the phase delay between the temperature and the pressure introduced by the interaction of the fluid with the regenerator. In the traveling wave case, the maximum in Figure 5.63 occurs for small hydraulic radii and lays at values that are close to the theoretic one of $\Delta T_{0}=2 / \pi \approx 0.64$ for the two difference regenerator lengths.

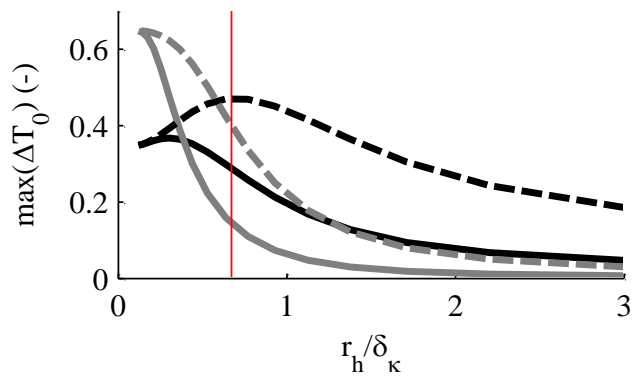

Figure 5.63: Maximum changes in mean temperature as a function of the ratio of the hydraulic radius and the thermal penetration depth for standing wave phasing (black lines) and traveling wave (gray line). Solid line represents results from the one-dimensional model with two cylinders and the dashed line represents results for ten cylinders. Red line indicates $r_{h} / \delta_{\kappa}=0.67$ corresponding to the reference geometry.

Another remarkable characteristic of the changes in mean temperature in Figure 5.62 is that the mean temperature profile in the case of the standing wave is not point symmetric around the origin. The maximum change of the mean temperature is higher than the minimum change in mean temperature. The one-dimensional model (dashed line) does 
not show this characteristic. One possible reason is streaming due to the boundary conditions, which only occurs for standing wave phasing. Streaming is not incorporated in the one-dimensional model, but the simulation show a large mean velocity. The streaming velocity increases linearly in time and becomes up to $10 \%$ of the velocity amplitude in these simulations. In order to reveal the effect of streaming, the one-dimensional model was recalculated including streaming. The necessary coefficients for the linear increasing mean velocity are obtained by fitting from the CFD simulation. The results are shown in Figure 5.62 with the dotted line. The profile of the one-dimensional model including streaming is closer to the CFD results. As opposed to Matveev [76], who reported large deviation between the one-dimensional model and experiments, only small deviations between the one-dimensional model and the simulation are found. Larger amplitude simulations would be needed in order to investigate the effect of vortices on the entrance effects, but cannot be conducted here due to the large amount of drift in pressure or velocity at high amplitude. In order to perform this study, more accurate boundary conditions would be needed that introduce less drift.

Another difference between the standing wave phasing and the traveling wave phasing is the heat that is transferred to the regenerator by the entrance effects. In an ideal case no heat would be transferred for a standing wave phasing as the pressure changes and the displacement are in phase. Whereas for a traveling wave heat would be transferred as the pressure changes and the displacement are out of phase.

The heat transferred to the two cylinders of the reference CFD geometry is given in Table 5.7 for both phases. As expected the heat pumped by the traveling wave is higher than the heat transferred by the standing wave. The thermodynamic cycle of the gas parcels starting at the position $x=0$ and $y=0$ are shown in Figure 5.64. The gray line corresponds to the thermodynamic cycle in the case of a traveling wave. As expected an area is enclosed and the same cycle occurs over the four periods shown in the figure. The black line shows the cycle that the corresponding gas parcel undergoes in the case of a standing wave. Due to streaming, the trajectory of the gas parcel is not closed and with this the thermodynamic cycle in the $p$ - $v$-diagram is also not closed. The area enclosed in the $p$ - $v$-diagram is small but non zero. Less heat is exchanged with the cylinders, as can be seen in Table 5.7. Furthermore, the heat transferred is not symmetric, which indicates that streaming is an important effect of the heat transfer in the case of the standing wave simulation.

Concluding, one can say that the thermal entrance effects on the regenerator are larger for traveling wave phasing than for standing wave phasing. Still, heat transfer also occurs for standing waves. For this phasing the heat transferred is increased by the streaming that occurs in these simulations. In the case of a traveling wave no streaming occurs, but due to phasing the entrance effects add a thermal load at the extremities off the regenerator. 


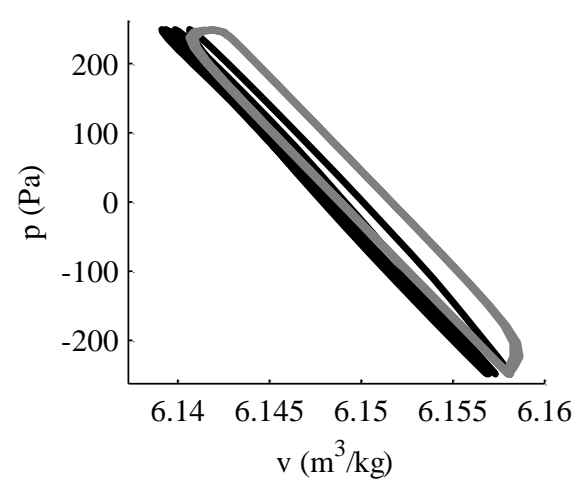

Figure 5.64: $\quad p$-v-diagram for the fluid parcels starting at position $x=0, y=0$ for the standing wave (black line) and traveling wave (gray line) phasing.

Table 5.7: Heat transferred to the fluid from both cylinders for the two different wave phasing. Values for the reference CFD geometry.

\begin{tabular}{ccc}
\hline Phasing & $\begin{array}{c}\boldsymbol{Q}_{\boldsymbol{L}, \boldsymbol{c y l}} \\
(\mathbf{m W})\end{array}$ & $\begin{array}{c}\boldsymbol{Q}_{\boldsymbol{R}, \boldsymbol{c y l}} \\
(\mathbf{m W})\end{array}$ \\
\hline Standing wave & 0,84 & $-0,14$ \\
Traveling wave & $-1,20$ & 1,24 \\
\hline
\end{tabular}

\subsubsection{Conclusion and recommendations}

In this section the entrance effects in stacked screen regenerators were investigated using CFD and compared to a one-dimensional model. The influence of the regenerator length and opening were studied. It is shown that the changes in mean temperature increase with an increasing number of cylinders and a smaller opening. In the limit of an ideal heat exchanger, the normalized temperature changes converge to $\Delta T_{0}=2 / \pi \approx$ 0.64 . The one-dimensional model slightly under predicts the changes in mean temperature for realistic regenerator dimensions.

For traveling wave phasing, heat is transferred from the fluid to the regenerator, adding an additional thermal load. In the standing wave case less heat is transferred by the entrance effects, as the entrance effects are mostly adiabatic. The heat transfer in the here proposed CFD standing wave model is due to numerical drifting that introduces streaming and with this convective heat transfer.

The simulations revealed the two-dimensional flow field around the cylinders, with a compression and wake area. At the simulated pressure amplitude, the two-dimensional 
effects are still small and the one-dimensional model predicts the temperature field qualitatively correctly. In future studies, the CFD model should be extended such that higher pressure amplitudes can be simulated, where for example vortex generation occurs. These two-dimensional effects can influence the entrance effects as they affect the heat transfer at the extremities of the regenerator, but cannot be predicted by the one-dimensional model. This indicates the need for further CFD simulation in order to get an in-depth understanding of the entrance effects at stacked screens experiencing higher amplitudes. To reach this goal, the acoustic boundary conditions have to be adapted such that less drift in pressure (traveling wave) or velocity (standing wave) occurs at high amplitudes.

The one-dimensional model can also be extended for a better prediction of the entrance effects. For example, in a heat exchanger regenerator assembly the phasing changes due to the different opening of the heat exchanger and regenerator. These effects can be implemented by also solving the one-dimensional momentum and continuity equations. This would allow quick performance checks of the thermoacoustic core, consisting of a regenerator and heat exchanger.

As the one-dimensional equation shows qualitative good agreement with the CFD simulation, this equation is a good tool for the design of thermoacoustic engines. 


\section{Concluding remarks}

In this work a step towards the numerical simulation of components of thermoacoustic devices with commercial CFD software is made. The conclusions and recommendations of the different chapters are shortly summarized in this chapter and some remarks on the improvements for broader future simulations of thermoacoustic components are given. Afterwards, an outlook on possible future paths in the simulation of thermoacoustic components with CFD is given.

In this work an impedance boundary condition is implemented into the commercially available code ANSYS Fluent [39], such that isolated components can be investigated. The boundary condition is validated successfully in one- and two-dimensional cases against analytical solutions from the low-reduced frequency approximation. Furthermore, the low reduced frequency solution is used in order to derive the optimal numerical parameters for thermoacoustic simulations and to give general rules of thumb for the spatial and time discretization.

In a second step these parameters are applied to the simulations of four different thermoacoustic cases in order to show that CFD can lead to a better understanding of phenomena that are not incorporated in the one-dimensional thermoacoustic equations.

Using the implemented ideal heat exchanger boundary condition, the two-dimensional streaming field inside the thermal buffer tube is revealed and the influence of the wall properties on the streaming pattern is estimated. The temperature field resulting from the different streaming patterns as well as the repercussions on the acoustic properties is shown. However, this case has also shown the limits of the implemented boundary condition, as the convected heat from the ideal heat exchanger into the thermal buffer tube could not be estimated. Furthermore, the ideal heat exchanger boundary condition at the hot heat exchanger had to be chosen fully reflective, otherwise an unwanted streaming over the boundary condition would occur. This means that for simulations of components with a temperature gradient, the boundary conditions have to be improved. A boundary condition that promises better results is the time domain impedance boundary condition that was implemented by van der Poel into ANSYS CFX [41] within the scope of his maser thesis [97] following the work of Huber et al. [98]. With this boundary condition it would be possible to investigate the influence of different wave phasing, which differs from a purely traveling wave in most thermoacoustic devices and is an important characteristic for the streaming inside of the thermal buffer tube.

The second study is conducted in the context of the miniaturization of thermoacoustic devices and investigates the geometric effects of increasingly sharp bends. With increasing sharpness additional reflections and dissipations are introduced, as well as a velocity component in the cross-direction, which is expected to influence nearby components such 
as heat exchangers. Nearby components are especially influenced when the Strouhal number $S t<1$, which means that vortex generation occurs. In general this study shows the strength of numerical CFD simulations in thermoacoustics. Only the implementation of an automatized process for meshing and simulating is time-consuming, but once this is done, the geometric parameters can be changed easily over a large parameter space, in order to get an in-depth understanding of the underlying flow phenomena.

The subsequent study in this thesis makes the link between the one-dimensional frequency domain thermoacoustic equations and the full time domain CFD, as it shows how the one-dimensional equations can be made more accurate when data from CFD is used. In the one-dimensional thermoacoustic equations the thermoacoustic functions are used in order to incorporate the three-dimensional effects. But often only the solution for simple pore geometries is used. In this work the thermoacoustic functions are calculated from CFD for a reduced model of a stacked screen regenerator, leading to more realistic values of the thermoacoustic functions. The thermal and the viscous thermoacoustic functions are estimated at the same time, as the pressure and the velocity are known at any point of the domain. Furthermore, the entrance effects are subtracted, as the flow field is known at every point inside of the domain. It is shown that the arrangement of the screens has a positive effect on the heat transfer inside the regenerator, while the viscous effects stay the same. With this study it is shown that not only large components like bends and the thermal buffer tube can be successfully simulated with CFD, but also small scale geometries like the ones inside the regenerator. A drawback of these simulations is that the nonreflecting boundary condition has to be placed sufficiently far from the object of consideration, otherwise the boundary interacts with the object under consideration. This increases the needed computational resources. Furthermore, a large drawback of the present acoustic boundary condition comes forth when small geometries are simulated, due to the boundary treatment, the spatial and the time discretization are linked. This leads to a very small time step and thus long simulation times, when small geometries are investigated. In the aforementioned characteristic boundary condition both discretizations are not linked, which allows for reduced simulation times.

In the last thermoacoustic study within this work, the entrance effects in a stacked screen regenerator are investigated. The mean temperature profile due to the entrance effects and the heat pumped at the end of the stacked screen regenerator are calculated for different phasing and amplitudes with CFD. Furthermore, a one-dimensional time dependent heat equation is used in order to predict the changes in mean temperature. In this one-dimensional time dependent heat transfer equation the thermal thermoacoustic function is used in order to estimate the heat transfer coefficient between the regenerator and the fluid. The results compare well with the CFD results, which is encouraging as the same approach might be extended to the momentum equation, such that a set of equations can be derived in the time domain. This allows the prediction of the startup process in a 
full thermoacoustic device with a dramatically reduced computational power requirement. This has been applied recently by Wang et al. [128].

From these studies one can deduce some important points for future thermoacoustic CFD simulations. First, thermoacoustic CFD simulations are relatively computationally demanding, as different spatial and time scales have to be resolved. This was the case for example for the thermal buffer tube which in some cases needed more than 300 periods to reach a steady periodic state. Another point that has to be taken into account is that the currently implemented acoustic boundary condition introduces drifting in some cases. In order to obtain high fidelity CFD results in these cases, a more accurate boundary condition has to be used, like the one implemented in ANSYS CFX by van der Poel [97]. However, the largest restriction from accurate CFD modeling in thermoacoustics comes from the turbulence that can occur at high amplitudes and for which no reliable model exists in the case of purely oscillating flow.

On the other side, when these points are taken into consideration and a parameter study is set up, the physical properties of the model can be varied easily, which makes it possible to investigate different influence factors and isolate them. Especially, the availability of the entire flow field makes the understanding of the underlying flow phenomena possible and is the strength of this method.

Finally, it can be concluded that simulations of components of thermoacoustic devices with commercial CFD are possible and that they will contribute to a better understanding of the flow phenomena inside of the respective components. This work paves the way towards the in-depth investigation of other components within the field of thermoacoustics. For example, based on this work different flow regimes inside of a jet pump could be distinguished and related to the jet pump performance [129]. With these in-depth investigations of components, the development of more efficient thermoacoustic devices will be possible and may lead to a broad commercial application of the thermoacoustic technology: the technology that puzzled me so much four years ago. 



\section{Bibliography}

[1] N. Rott, "Thermoacoustics," Advances in Applied Mechanics, vol. 20, pp. 135-175, 1980.

[2] S. J. W. Rayleigh, The Theory of Sound, 2nd edition ed., Dover Publications, 1945.

[3] F. Joos, "Thermoakustik von Brennkammern," in Technische Verbrennung, Springer Berlin Heidelberg, 2006, pp. 737-774.

[4] J. C. Roman, "Nonlinear behavior of the thermoacoustic instabilities in the limousine combustor," University of Twente, Enschede, 2013.

[5] N. Rott, "Damped and thermally driven acoustic oscillations in wide and narrow tubes," Zeitschrift für angewandte Mathematik und Physik ZAMP, vol. 20, no. 2, pp. 230-243, 1969.

[6] G. W. Swift, Thermoacoustics: a unifying perspective for some engines and refrigerators, Acoustical Society of America through the American Institute of Physics, 2002.

[7] B. Ward, J. Clark and G. Swift, Design Environment for Low-Amplitude ThermoAcoustic Energy Conversion (DeltaEC) User's Guide (Version 6.2), Los Alamos National Laboratory, 2008.

[8] A. Abduljalil, Z. Yu and A. Jaworski, "Design and experimental validation of looped-tube thermoacoustic engine," Journal of Thermal Science, vol. 20, no. 5, pp. 423-429, 2011.

[9] S. Backhaus and G. W. Swift, "A thermoacoustic-Stirling heat engine: Detailed study," Journal of the Acoustical Society of America, vol. 107, p. 19, 2000.

[10] M. Chen and Y. Ju, "Effect of different working gases on the performance of a small thermoacoustic Stirling engine," International Journal of Refrigeration, vol. 51, pp. 41-51, 2015.

[11] T. J. Hofler, "Thermoacoustic Refrigerator Design and Performance," University of California, San Diego, 1986.

[12] G. Swift, "Thermoacoustic engines," Journal of the Acoustical Society of America, vol. 84, pp. 1145-1180, 1988.

[13] P. H. Ceperley, "A pistonless Stirling engine - The traveling wave heat engine," The Journal of the Acoustical Society of America, vol. 66, no. 5, pp. 1508-1513, 1979. 
[14] S. Backhaus and G. W. Swift, "A thermoacoustic Stirling heat engine," Nature, vol. 399, no. 6734, pp. 335-338, 1999.

[15] D. Blok, "Thermo-acoustic system". US Patent 6314740 B1, 13112001.

[16] M. Poese, "Thermoacoustics," in Handbook of Climate Change Mitigation, W. Chen, J. Seiner, T. Suzuki and M. Lackner, Eds., Springer US, 2012, pp. 18211848.

[17] R.-1. Chen and S. L. Garrett, "Solar/Heat Driven Thermoacoustic Engine," in Proceedings of 16th International Congress on Acoustics, 1998.

[18] K. D. Blok, "Low operating temperature integral thermo acoustic devices for solar cooling and waste heat recovery," The Journal of the Acoustical Society of America, vol. 123, no. 5, pp. 3541-3541, 2008.

[19] R. Ali, S. Garrett, J. Smith and D. Kotter, "Thermoacoustic thermometry for nuclear reactor monitoring," Instrumentation Measurement Magazine, IEEE, vol. 16, no. 3, pp. 18-25, June 2013.

[20] K. d. Blok, "On the design of near atmospheric air operated thermoacoustic engines," Aster Thermoacoustics, 2013.

[21] S. L. Garrett and M. E. Poese, "There's (still) plenty of room at the bottom," Procedia Engineering, vol. 56, pp. 842-848, 2013.

[22] J.-A. Mumith, C. Makatsoris and T. Karayiannis, "Design of a thermoacoustic heat engine for low temperature waste heat recovery in food manufacturing: A thermoacoustic device for heat recovery," Applied Thermal Engineering, vol. 65, no. 1-2, pp. 588-596, 2014.

[23] M. Tijani and S. Spoelstra, "A Hot Air Driven Thermoacoustic-Stirling Engine," Applied Thermal Engineering, vol. 61, no. 2, pp. 866-870, 2013.

[24] D. L. Gardner and C. Q. Howard, "Waste-heat-driven thermoacoustic engine and refrigerator," in Proceedings of ACOUSTICS 2009, Adelaide, 2009.

[25] P. Aben, P. Bloemen and J. Zeegers, "2-D PIV measurements of oscillatory flow around parallel plates," Experiments in Fluids, vol. 46, no. 4, pp. 631-641, 2009.

[26] L. Shi, Z. Yu and A. J. Jaworski, "Investigation into the Strouhal numbers associated with vortex shedding from parallel-plate thermoacoustic stacks in oscillatory flow conditions," European Journal of Mechanics - B/Fluids, vol. 30, no. 2, pp. 206-217, 2011.

[27] P. Blanc-Benon, E. Besnoin and O. Knio, "Experimental and computational visualization of the flow field in a thermoacoustic stack," Comptes Rendus Mecanique, vol. 331, p. 17-24, 2003. 
[28] N. Cao, J. R. Olson, G. W. Swift and S. Chen, "Energy flux density in a thermoacoustic couple," The Journal of the Acoustical Society of America, vol. 99, no. 6, pp. 3456-3464, 1996.

[29] A. Worlikar, O. Knio and R. Klein, "Numerical simulation of a thermoacoustic refrigerator," ESAIM, vol. 1, p. 363-375, 1996.

[30] A. S. Worlikar and O. M. Knio, "Numerical Simulation of a Thermoacoustic Refrigerator: I. Unsteady Adiabatic Flow around the Stack," Journal of Computational Physics, vol. 127, no. 2, pp. 424-451, 1996.

[31] A. S. Worlikar, O. M. Knio and R. Klein, "Numerical Simulation of a Thermoacoustic Refrigerator: II. Stratified Flow around the Stack," Journal of Computational Physics, vol. 144, no. 2, pp. 299-324, 1998.

[32] A. S. Worlikar, "Numerical study of oscillatory flow and heat transfer in a loaded thermoacoustic stack," Numerical Heat Transfer, Part A: Applications, vol. 35, pp. 49-65, 1999.

[33] E. Besnoin and O. M. Knio, "Numerical Study of Thermoacoustic Heat Exchangers," Acta Acustica united with Acustica, vol. 90, pp. 432-444(13), 2004.

[34] E. Besnoin and O. Knio, "Numerical study of thermoacoustic heat exchangers in the thin plate limit," Numerical Heat Transfer; Part A: Applications, vol. 40, no. 5, pp. 445-471, 2001.

[35] D. Marx and P. Blanc-Benon, "Numerical calculation of the temperature difference between the extremities of a thermoacoustic stack plate," Cryogenics, vol. 45, no. 3, pp. 163-172, 2005.

[36] D. Marx and P. Blanc-Benon, "Numerical simulation of stack-heat exchangers coupling in a thermoacoustic refrigerator," American Institute of Aeronautics and Astronautics Journal, vol. 42, no. 7, pp. 1338-1347, 2004.

[37] D. Marx and P. Blanc-Benon, "Computation of the mean velocity field above a stack plate in a thermoacoustic refrigerator," Comptes Rendus Mecanique, vol. 332, no. 11, pp. 867-874, 2004.

[38] D. Marx and P. Blanc-Benon, "Computation of the temperature distortion in the stack of a standing-wave thermoacoustic refrigerator," Journal of the Acoustical Society of America, vol. 118, no. 5, pp. 2993-2999, 2005.

[39] "ANSYS FLUENT 14.0 User's Guide," 275 Technology Drive Canonsburg, PA 15317, 2013.

[40] L. Zoontjens, C. Howard, A. Zander and B. Cazzolato, "Numerical study of flow and energy fields in thermoacoustic couples of non-zero thickness," International Journal of Thermal Sciences, vol. 48, no. 4, pp. 733-746, 2009. 
[41] "ANSYS CFX-Pre User's Guide," 275 Technology Drive Canonsburg, PA 15317, 2013.

[42] J. Lycklama à Nijeholt, M. Tijani, S. Spoelstra, M. Loginov and A. Kuczaj, "CFD modeling of streaming phenomena in a torus-shaped thermoacoustic engine," Proceedings of the 19th International Congress on Sound and Vibration, vol. 1, pp. $1-8,2012$.

[43] C. Scalo, S. K. Lele and L. Hesselink, "Linear and nonlinear modelling of a theoretical travelling-wave thermoacoustic heat engine," Journal of Fluid Mechanics, vol. 766, pp. 368-404, 32015.

[44] F. Zink, J. Vipperman and L. Schaefer, "CFD simulation of a thermoacoustic engine with coiled resonator," International Communications in Heat and Mass Transfer, vol. 37, no. 3, pp. 226-229, 2010.

[45] W. Geller, Thermodynamik für Maschinenbauer, Springer-Verlag Berlin Heidelberg, 2006.

[46] P. in 't Panhuis, "Mathematical Aspects of Thermoacoustics," Eindhoven University of Technologie, Eindhoven, 2009.

[47] Y. Cengel and R. Turner, Fundamentals of Thermal-Fluid Sciences, McGraw-Hill Education, 2001.

[48] D. Gedeon, "Sage, User's Guide, Stirling, Pulse-Tube and Low-T Cooler Model Classes," 2013.

[49] H. Oertel jr., M. Böhle and U. Dohrmann, Strömungsmechanik, 5., überarbeitete und erweiterte Auflage ed., Wiesbaden: Vieweg+Teubner, 2009.

[50] D. Blackstock, Fundamentals of physical acoustics, Wiley-interscience, 2000.

[51] T. Butz, Fouriertransformation für Fußgänger, Vieweg+Teubner Verlag, 2009.

[52] W. P. Arnott, H. E. Bass and R. Raspet, "General formulation of thermoacoustics for stacks having arbitrarily shaped pore cross sections," The Journal of the Acoustical Society of America, vol. 90, no. 6, pp. 3228-3237, 1991.

[53] A. Craggs and J. Hildebrandt, "Effective densities and resistivities for acoustic propagation in narrow tubes," Journal of Sound and Vibration, vol. 92, no. 3, pp. 321-331, 1984.

[54] M. E. Hayden and G. W. Swift, "Thermoacoustic relaxation in a pin-array stack," The Journal of the Acoustical Society of America, vol. 102, no. 5, pp. 2714-2722, 1997.

[55] C. Jensen, "Computational Thermoacoustics in Fibrous Stacks," The University of Mississippi, 2009. 
[56] Y. Ueda, T. Kato and C. Kato, "Experimental evaluation of the acoustic properties of stacked-screen regenerators," The Journal of the Acoustical Society of America, vol. 125, no. 2, pp. 780-786, 2009.

[57] H. Tijdeman, "On the propagation of sound waves in cylindrical tubes," Journal of Sound and Vibration, vol. 39, no. 1, pp. 1-33, 1975.

[58] M. Hannink, "Acoustic Resonators for the Reduction of Sound Radiation and Transmission," University of Twente, Enschede, 2007.

[59] M. Nijhof, "Viscothermal wave propagation," University of Twente, Enschede, 2010.

[60] J. H. Ferziger and M. Perić, Numerische Strömungsmechanik, Berlin Heidelberg New York: Springer-Verlag Berlin Heidelberg, 2008.

[61] E. Laurien and H. Oertel, Numerische Strömungsmechanik, Vieweg+Teubner, 2009.

[62] "ANSYS FLUENT 14.0 Theory Guide," 275 Technology Drive Canonsburg, PA 15317, 2013.

[63] F. Zink, J. Vipperman and L. Schaefer, "CFD simulation of thermoacoustic cooling," International Journal of Heat and Mass Transfer, vol. 53, no. 19-20, pp. 3940-3946, 2010.

[64] G. Yu, W. Dai and E. Luo, "CFD simulation of a $300 \mathrm{~Hz}$ thermoacoustic standing wave engine," Cryogenics, vol. 50, no. 9, pp. 615-622, 2010.

[65] J. Xu, D. Zhao, F. Chen and S. Xu, "Numerical Simulating for Turbulent Heat Transfer of Thermoacoustic Cooler," Procedia Engineering, vol. 16, no. 0, pp. 789795, 2011.

[66] Y. Banjare, R. Sahoo and S. Sarangi, "CFD simulation and experimental validation of a GM type double inlet pulse tube refrigerator," Cryogenics, vol. 50, no. 4, pp. 271-280, 2010.

[67] T. Ashwin, G. Narasimham and S. Jacob, "CFD analysis of high frequency miniature pulse tube refrigerators for space applications with thermal nonequilibrium model," Applied Thermal Engineering, vol. 30, no. 2-3, pp. 152-166, 2010.

[68] Z. Liao and H. Wong, "A transmitting boundary for the numerical simulation of elastic wave propagation," International Journal of Soil Dynamics and Earthquake Engineering, vol. 3, no. 4, pp. 174-183, 1984.

[69] W. Polifke, C. Wall and P. Moin, "Partially reflecting and non-reflecting boundary conditions for simulation of compressible viscous flow," Journal of Computational Physics, vol. 213, p. 437-449, 2006. 
[70] "ANSYS FLUENT 14.0 UDF Manual," 275 Technology Drive Canonsburg, PA 15317, 2013.

[71] T. Poinsot and D. Veynante, Theoretical and Numerical Combustion, 3nd ed., Bordeaux, 2005.

[72] J. H. Ferziger and M. Peric, Physikalische Grundlagen der Strömungen, Springer Berlin Heidelberg, 2008, pp. 1-24.

[73] M. F. Hamilton and D. T. Blackstock, Nonlinear Acoustic, Academic Press, 1998.

[74] T. Poinsot and S. Lele, "Boundary conditions for direct simulations of compressible viscous flows," Journal of Computational Physics, vol. 101, no. 1, pp. 104-129, 1992.

[75] P. J. Storch, R. Radebaugh and J. E. Zimmerman, Analytical model for the refrigeration power of the orifice pulse tube refrigerator, National Institute of Standards and Technology (U.S.), 1990.

[76] K. I. Matveev, G. W. Swift and S. Backhaus, "Temperatures near the interface between an ideal heat exchanger and a thermal buffer tube or pulse tube," International Journal of Heat and Mass Transfer, vol. 49, pp. 868-878, 2006.

[77] V. Gusev, P. Lotton, H. Bailliet, S. Job and M. Bruneau, "Thermal wave harmonics generation in the hydrodynamical heat transport in thermoacoustics," The Journal of the Acoustical Society of America, vol. 109, no. 1, pp. 84-90, 2001.

[78] K. I. Matveev, G. W. Swift and S. Backhaus, "Analytical solution for temperature profiles at the ends of thermal buffer tubes," International Journal of Heat and Mass Transfer, vol. 50, pp. 897-901, 2007.

[79] L. Rayleigh, "On the Circulation of Air Observed in Kundt's Tubes, and on Some Allied Acoustical Problems," Philosophical Transactions of the Royal Society of London, vol. 175, pp. pp. 1-21, 1884.

[80] N. Rott, "The influence of heat conduction on acoustic streaming," Zeitschrift für angewandte Mathematik und Physik ZAMP, vol. 25, no. 3, pp. 417-421, 1974.

[81] H. Bailliet, V. Gusev, R. Raspet and R. A. Hiller, "Acoustic streaming in closed thermoacoustic devices," The Journal of the Acoustical Society of America, vol. 110, no. 4, pp. 1808-1821, 2001.

[82] M. F. Hamilton, Y. A. Ilinskii and E. A. Zabolotskaya, "Acoustic streaming generated by standing waves in two-dimensional channels of arbitrary width," The Journal of the Acoustical Society of America, vol. 113, no. 1, pp. 153-160, 2003.

[83] J. Lee, P. Kittel, K. Timmerhaus and R. Radebaugh, "Flow patterns intrinsic to the pulse tube refrigerator," in Proceedings of the 7th International Cryocooler Conference, Santa Fe, 1992. 
[84] J. Olson and G. Swift, "Acoustic streaming in pulse tube refrigerators: tapered pulse tubes," Cryogenics, vol. 37, no. 12, pp. 769-776, 1997.

[85] S. Boluriaan and P. Morris, "Acoustic streaming: from Rayleigh to today," International Journal of Aeroacoustics, vol. 2, no. 3, pp. 255-292, 2003.

[86] C. Gu, J. Tang, J. Wang and Y. Zhou, "Advance in research of several types of streaming of pulse tube refrigerators," Science China Technological Sciences, vol. 56, no. 11, pp. 2690-2701, 2013.

[87] Y. He, C. Zhao, W. Ding and W. Yang, "Two-dimensional numerical simulation and performance analysis of tapered pulse tube refrigerator," Applied Thermal Engineering, vol. 27, no. 11-12, pp. 1876-1882, 2007.

[88] M. K. Aktas and B. Farouk, "Numerical simulation of acoustic streaming generated by finite-amplitude resonant oscillations in an enclosure," The Journal of the Acoustical Society of America, vol. 116, no. 5, pp. 2822-2831, 2004.

[89] X. Zhang, L. Qiu, Z. Gan and Y. He, "CFD study of a simple orifice pulse tube cooler," Cryogenics, vol. 47, no. 5-6, pp. 315-321, 2007.

[90] C. Gu, Y. Zhou, J. Wang, W. Ji and Q. Zhou, "CFD analysis of nonlinear processes in pulse tube refrigerators: Streaming induced by vortices," International Journal of Heat and Mass Transfer, vol. 55, no. 25-26, pp. 7410-7418, 2012.

[91] D. S. Antao and B. Farouk, "Computational fluid dynamics simulations of an orifice type pulse tube refrigerator: Effects of operating frequency," Cryogenics, vol. 51, no. 4, pp. 192-201, 2011.

[92] A. Berson, G. Poignand, P. Blanc-Benon and G. Comte-Bellot, "Nonlinear temperature field near the stack ends of a standing-wave thermoacoustic refrigerator," International Journal of Heat and Mass Transfer, vol. 54, no. 21-22, pp. 4730-4735, 2011.

[93] J. Liu and S. L. Garrett, "Relationship between Nusselt number and the thermoviscous (Rott) functions," The Journal of the Acoustical Society of America, vol. 119, no. 3, pp. 1457-1462, 2006.

[94] Y. A. Cengel, Heat \& Mass Transfer: A Practical Approach, third ed., McGrawHill Education (India) Pvt Limited, 2006.

[95] J. H. So, G. W. Swift and S. Backhaus, "An internal streaming instability in regenerators," The Journal of the Acoustical Society of America, vol. 120, no. 4, pp. 1898-1909, 2006.

[96] K. I. Matveev, S. Backhaus and G. W. Swift, "The effect of gravity on heat transfer by rayleigh streaming in pulse tubes and thermal buffer tubes," ASME 2004 International Mechanical Engineering Congress and Exposition, vol. 3, pp. 7-12, 2004. 
[97] B. van der Poel, "Time-domain impedance boundary conditions in computational fluid dynamics for use in thermoacoustic modeling," University of Twente, Enschede, 2013.

[98] A. Huber, P. Romann and W. Polifke, "Filter-Based Time-Domain Impedance Boundary Conditions for CFD Applications," ASME Conference Proceedings, vol. 2008, no. 43130, pp. 901-911, 2008.

[99] G. W. Swift and S. Backhaus, "The pulse tube and the pendulum," The Journal of the Acoustical Society of America, vol. 126, no. 5, pp. 2273-2284, 2009.

[100] M.-X. Francois, P. T. Le and A. Betrancourt, "Thermoacoustic machine having an electric feedback loop". Patent WO 2011098735 A3, 24 November 2011.

[101] R. Dyson and G. Bruder, "Alpha-stream convertor". Patent WO2013126206 A1, 29 August 2013.

[102] J. A. Lycklama, M. Tijani and S. Spoelstra, "Simulation of a traveling-wave thermoacoustic engine using computational fluid dynamics," The Journal of the Acoustical Society of America, vol. 118, p. 2265, 2005.

[103] W. Rostafinski, "Monograph on propagation of sound waves in curved ducts," NASA reference Publication, Cleveland, 1991.

[104] S. Dequand, S. J. Hulshoff, Y. Aurégan, J. Huijnen, R. t. Riet, L. J. van and A. Hirschberg, "Acoustics of 90 degree sharp bends. Part I: Low-frequency acoustical response," Acta Acustica united with Acustica, vol. 89, no. 6, pp. 1025-1037, 2003.

[105] S. Dequand, S. J. Hulshoff, Y. Aurégan, J. Huijnen, R. T. Riet, L. J. van and A. Hirschberg, "Acoustics of 90 degree sharp bends. Part II: Low-frequency aeroacoustical response," Acta Acustica united with Acustica, vol. 90, no. 1, pp. 1323, 2004.

[106] M. Iguchi, M. Ohmi and K. Maegawa, "Analysis of Free Oscillating Flow in a UShaped Tube," Bulletin of JSME, vol. 25, no. 207, pp. 1398-1405, sep 1982.

[107] J. R. Olson and G. W. Swift, "Energy dissipation in oscillating flow through straight and coiled pipes," The Journal of the Acoustical Society of America, vol. 100, no. 4, pp. 2123-2131, 1996.

[108] R. Radebaugh, "Cryocoolers: the state of the art and recent developments," Journal of Physics: Condensed Matter, vol. 21, no. 16, pp. 164-219, 2009.

[109] I. J. Sobey, "Oscillatory flows at intermediate Strouhal number in asymmetric channels," Journal of Fluid Mechanics, vol. 125, pp. 359-373, 121982.

[110] G. W. Swift and W. C. Ward, "Simple harmonic analysis of regenerators," Journal of Thermophysics and Heat Transfer, vol. 10, no. 4, pp. 652-662, 1996. 
[111] L. A. Wilen, "Measurements of scaling properties for acoustic propagation in a single pore," The Journal of the Acoustical Society of America, vol. 101, no. 3, pp. 1388-1397, 1997.

[112] L. A. Wilen, "Measurements of thermoacoustic functions for single pores," The Journal of the Acoustical Society of America, vol. 103, no. 3, pp. 1406-1412, 1998.

[113] G. Petculescu and L. A. Wilen, "High-amplitude thermoacoustic effects in a single pore," The Journal of the Acoustical Society of America, vol. 109, no. 3, pp. 942948, 2001.

[114] G. Petculescu and L. A. Wilen, "Thermoacoustics in a single pore with an applied temperature gradient," The Journal of the Acoustical Society of America, vol. 106, no. 2, pp. 688-694, 1999.

[115] F. C. Bannwart, G. Penelet, P. Lotton and J.-P. Dalmont, "Measurements of the impedance matrix of a thermoacoustic core: Applications to the design of thermoacoustic engines," The Journal of the Acoustical Society of America, vol. 133, no. 5, pp. 2650-2660, 2013.

[116] S. Costa, H. Barrutia, J. A. Esnaola and M. Tutar, "Numerical study of the heat transfer in wound woven wire matrix of a Stirling regenerator," Energy Conversion and Management, vol. 79, pp. 255-264, 2014.

[117] M. Lewis, T. Kuriyama, F. Kuriyama and R. Radebaugh, "Measurement of Heat Conduction through Stacked Screens," in Advances in Cryogenic Engineering, vol. 43, 1998, pp. 1611-1618.

[118] P. Kittel, "The Temperature Profile within Pulse Tubes," Advances in Cryogenic Engineering, vol. 43, pp. 1927-1932, 1998.

[119] C. H. K. Williamson, "Sinusoidal flow relative to circular cylinders," Journal of Fluid Mechanics, vol. 155, pp. 141-174, 1985.

[120] H.-w. An, L. Cheng, M. Zhao and G. h. Dong, "Numerical simulation of the oscillatory flow around two cylinders in tandem," Journal of Hydrodynamics, Ser. B, vol. 18, no. 3, pp. 191-197, 2006.

[121] P. Anagnostopoulos and C. Dikarou, "Numerical simulation of viscous oscillatory flow past four cylinders in square arrangement," Journal of Fluids and Structures, vol. 27, no. 2, pp. 212-232, 2011.

[122] P. Anagnostopoulos, A. Koutras and S. Seitanis, "Numerical study of oscillatory flow past two cylinders in oblique arrangement," in Proceedings of 11th International Conference on Computational Methods and Experimental Measurements, Halkidiki, 2003. 
[123] P. Anagnostopoulos, A. Koutras and S. Seitanis, "Numerical study of oscillatory flow past a pair of cylinders in a side-by-side arrangement," in ASME 24th International Conference on Offshore Mechanics and Arctic Engineering, 2005.

[124] G. Mozurkewich, "Heat transfer from a cylinder in an acoustic standing wave," The Journal of the Acoustical Society of America, vol. 98, no. 4, pp. 2209-2216, 1995.

[125] A. Gopinath and D. R. Harder, "An experimental study of heat transfer from a cylinder in low-amplitude zero-mean oscillatory flows," International Journal of Heat and Mass Transfer, vol. 43, no. 4, pp. 505-520, 2000.

[126] H. Iwai, T. Mambo, N. Yamamoto and K. Suzuki, "Laminar convective heat transfer from a circular cylinder exposed to a low frequency zero-mean velocity oscillating flow," International Journal of Heat and Mass Transfer, vol. 47, no. 21, pp. 4659-4672, 2004.

[127] C. Wu, Thermodynamics and Heat Powered Cycles: A Cognitive Engineering Approach, Nova Science Publishers, Inc., 2007.

[128] K. Wang, D. Sun, J. Zhang, J. Zou, K. Wu, L. Qiu and Z. Huang, "Numerical simulation on onset characteristics of traveling-wave thermoacoustic engines based on a time-domain network model," International Journal of Thermal Sciences, vol. 94, pp. 61-71, 2015.

[129] J. P. Oosterhuis, S. Bühler, D. Wilcox and T. H. van der Meer, "A numerical investigation on the vortex formation and flow separation of the oscillatory flow in jet pumps," J. Acoust. Soc. Am., vol. 137, pp. 1722-1731, 2015. 


\section{Appendices}

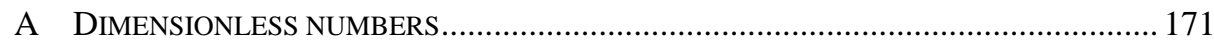

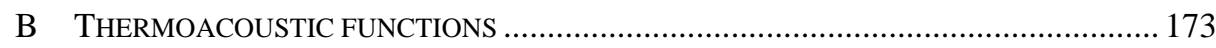

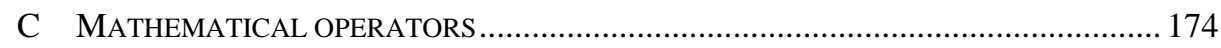

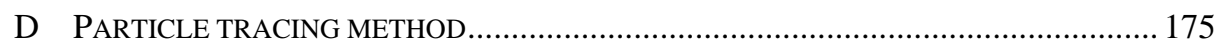

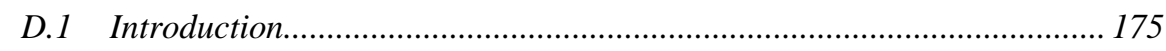

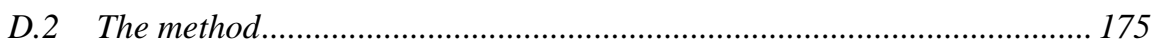

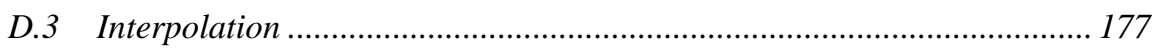

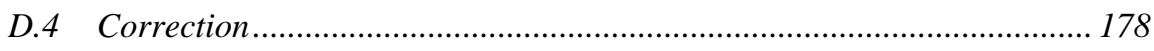

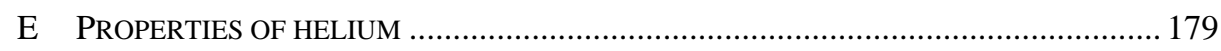

E.1 Temperature dependent properties .................................................. 180

F SHAKING RESONATOR - CHANGE IN COORDINATE SYSTEM ................................. 181

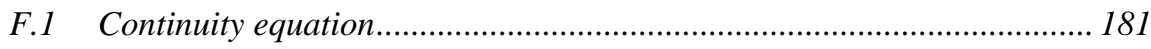

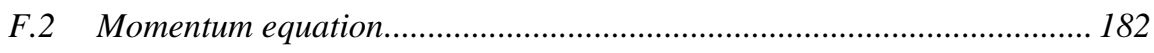

F.3 Energy Equation ............................................................................ 183

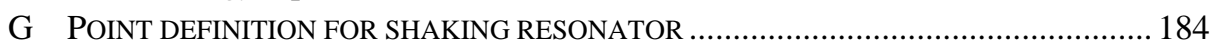

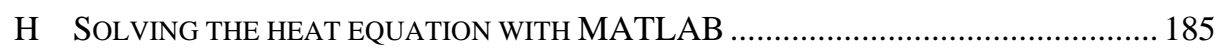

I INFLUENCE OF ERRORS ON CALCULATION OF THERMOACOUSTIC FUNCTIONS ..... 187

I.1 Rounding errors .............................................................................. 188

I.2 Errors on acoustic constants............................................................... 189

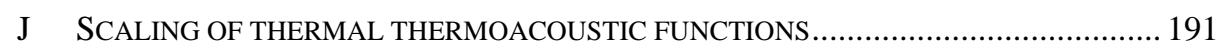

K THERMAL BUFFER TUBE BOUNDARY CONDITIONS ................................................. 193

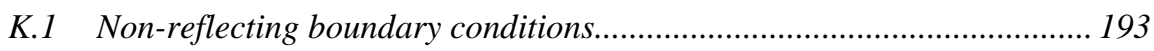

K.2 Reflecting boundary condition ............................................................ 194

K.3 Variable monitor point......................................................................... 196

L THERMAL BUFFER TUBE MESH REFINEMENT ...................................................... 198

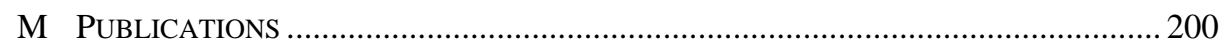





\section{A Dimensionless numbers}

CFL number: is a measure for the number of cells a acoustic wave propagates within one time step.

$$
\mathrm{CFL}=\mathrm{c}_{0} \frac{\Delta \mathrm{t}}{\Delta \mathrm{x}}
$$

Drive ratio: ratio of the pressure amplitude to the mean pressure. Is a measure for the non-linearity of acoustic waves.

$$
D_{r}=\frac{p_{1}}{p_{0}}
$$

Fourier number: ratio of conductive transport rate to the quantity storage rate.

$$
\mathrm{Fo}=\frac{\mathrm{kt}}{\rho \mathrm{c}_{\mathrm{p}} \mathrm{L}^{2}}
$$

Reduced frequency: ratio of tube radius to wavelength.

$$
k=\frac{\omega R}{c_{0}}
$$

Keulegan-Carpenter number: ratio of the drag forces to the inertial forces.

$$
\mathrm{KC}=\frac{\mathrm{u}_{1}}{\mathrm{fD}}
$$

Lautrec number: ratio of hydraulic radius $r_{h}$ and thermal penetration depth.

$$
L c=\frac{r_{h}}{\delta_{\kappa}}
$$

Viscous Lautrec number: ratio of hydraulic radius $r_{h}$ and viscous penetration depth.

$$
L c_{v}=\frac{r_{h}}{\delta_{v}}
$$

Mach number: ratio of the fluids to the unperturbed speed of sound.

$$
M=\frac{v}{c_{0}}
$$

Prandtl number: the ratio of momentum diffusivity to thermal diffusivity.

$$
\operatorname{Pr}=\frac{v}{\alpha}=\frac{c_{p} \mu}{k}
$$

Reynolds number: gives a measure of the ratio of inertial forces to viscous forces.

$$
R e=\frac{\rho u_{1} L}{\mu}=\frac{u_{1} L}{v}
$$

Shear wave number: ratio between the length scale (tube radius) and the viscous boundary layer thickness. Is a measure for the ratio between the inertial effects and the viscous effects in the gas.

$$
s=R \sqrt{\frac{\rho_{0} \omega}{\mu}}
$$


Thermal shear wave number: ratio between the length scale (tube radius) and the thermal boundary layer thickness.

$$
s_{t}=R \sqrt{\frac{\rho_{0} \omega c_{p}}{\lambda}}
$$

Strouhal number: is a measure for vortex generation.

$$
\mathrm{St}=\frac{\mathrm{Rf}}{\mathrm{u}}
$$

Ratio of specific heats: ratio of the heat capacity at constant pressure to heat capacity at constant volume.

$$
\gamma=\frac{C_{p}}{C_{v}}
$$

Dimensionless radius: ratio of the radius to the half spacing.

$$
\chi=\frac{\mathrm{R}}{\mathrm{h}}
$$




\section{B Thermoacoustic functions}

For some geometries the thermoacoustic functions can be calculated analytically. The differential equation (2.43) can be solved assuming no-slip or isothermal boundaries at the walls for the viscous and the thermal part, respectively. A summary of these cases is given here [7]:

- Boundary layer approximation with the hydraulic radius $r_{h}$ :

$$
f_{j}=\frac{(1-i) \delta_{j}}{2 r_{h}}, \quad \text { for } j=\kappa \text { or } v
$$

- Parallel plates of the half spacing $y_{0}$ :

$$
f_{j}=\frac{\tanh \left((1+i) \frac{y_{0}}{\delta_{j}}\right)}{(1+i) \frac{y_{0}}{\delta_{j}}}, \quad \text { for } j=\kappa \text { or } v
$$

- Rectangular pores of dimension $2 a \times 2 b$ :

$$
\begin{gathered}
f_{j}=1-\frac{64}{\pi^{4}} \sum_{m, n \text { odd }} \frac{1}{m^{2} n^{2}\left(1-i \frac{\pi^{2} \delta_{j}^{2}}{8 a^{2} b^{2}}\left(b^{2} m^{2}+a^{2} n^{2}\right)\right)}, \\
\text { for } j=\kappa \text { or } v
\end{gathered}
$$

- $\quad$ Circular pores of radius $R$ :

$$
f_{j}=\frac{2 J_{1}\left[(i-1) \frac{R}{\delta_{j}}\right]}{(i-1) \frac{R}{\delta_{j}} J_{0}\left[(i-1) \frac{R}{\delta_{j}}\right]}, \quad \text { for } j=\kappa \text { or } \nu
$$

with $J_{n}$ the Bessel function of the first kind of the order $n$.

- Pin array stack:

$$
f_{j}=-\frac{2 \alpha_{i}}{\alpha_{0}^{2}-\alpha_{i}^{2}} \frac{Y_{1}\left[\alpha_{o}\right] J_{1}\left[\alpha_{i}\right]-J_{1}\left[\alpha_{o}\right] Y_{1}\left[\alpha_{i}\right]}{Y_{1}\left[\alpha_{o}\right] J_{0}\left[\alpha_{i}\right]-J_{1}\left[\alpha_{o}\right] Y_{0}\left[\alpha_{i}\right]}, \quad \text { for } j=\kappa \text { or } v
$$

where $\alpha_{i}=(i-1) r_{i} / \delta_{j}$ and $\alpha_{o}=(i-1) r_{o} / \delta_{j}$. Furthermore, $Y_{n}$ is the Bessel function of the second kind of order $\mathrm{n}, r_{i}$ is the pin radius and $r_{0}^{2}=$ $\sqrt{3} /(2 \pi) \cdot\left(2 y_{0}\right)^{2}$ with $2 y_{0}$ the center to center distance of two pins in a hexagonal arrangement. 


\section{Mathematical operators}

Table C.1: $\quad$ Table with operators in Cartesian and cylindrical coordinates for scalar field $f$ and vector field $\mathrm{A}=A_{x} \vec{x}+A_{y} \vec{y}+A_{z} \vec{z}$ and $A=A_{r} \vec{r}+A_{\varphi} \vec{\varphi}+A_{z} \vec{z}$ respectively

\section{Cartesian coordinates Cylindrical coordinates}

Gradient $\nabla f \quad \frac{\partial f}{\partial x} \vec{x}+\frac{\partial f}{\partial y} \vec{y}+\frac{\partial f}{\partial z} \vec{z} \quad \frac{\partial f}{\partial r} \vec{r}+\frac{\partial f}{\partial \varphi} \vec{\varphi}+\frac{\partial f}{\partial z} \vec{z}$

Divergence $\nabla \cdot A \quad \frac{\partial A_{x}}{\partial x}+\frac{\partial A_{y}}{\partial y}+\frac{\partial A_{z}}{\partial z} \quad \frac{1}{r} \frac{\partial r A_{r}}{\partial r}+\frac{1}{r} \frac{\partial A_{\varphi}}{\partial \varphi}+\frac{\partial A_{z}}{\partial z}$

$$
\left(\frac{\partial A_{z}}{\partial y}-\frac{\partial A_{y}}{\partial z}\right) \vec{x} \quad\left(\frac{1}{r} \frac{\partial A_{z}}{\partial \varphi}-\frac{\partial A_{\varphi}}{\partial z}\right) \vec{r}
$$

$\operatorname{Curl} \nabla \times A$

$$
\begin{array}{ll}
+\left(\frac{\partial A_{x}}{\partial z}-\frac{\partial A_{z}}{\partial x}\right) \vec{y} & +\left(\frac{\partial A_{r}}{\partial z}-\frac{\partial A_{z}}{\partial r}\right) \vec{\varphi} \\
+\left(\frac{\partial A_{y}}{\partial x}-\frac{\partial A_{x}}{\partial y}\right) \vec{z} & +\frac{1}{r}\left(\frac{\partial r A_{\varphi}}{\partial r}-\frac{\partial A_{r}}{\partial \varphi}\right) \vec{z}
\end{array}
$$

Laplace operator

$\Delta A=\nabla^{2} A$

$$
\frac{\partial^{2} f}{\partial x^{2}}+\frac{\partial^{2} f}{\partial y^{2}}+\frac{\partial^{2} f}{\partial z^{2}}
$$

$\frac{1}{r} \frac{\partial}{\partial r}\left(r \frac{\partial f}{\partial r}\right)+\frac{1}{r^{2}} \frac{\partial^{2} f}{\partial \varphi^{2}}+\frac{\partial^{2} f}{\partial z^{2}}$

$$
\begin{array}{cll}
\text { Vector Laplacian } & & \left(\Delta A_{r}-\frac{A_{r}}{r^{2}}-\frac{2}{r^{2}} \frac{\partial A_{\varphi}}{\partial \varphi}\right) \vec{r} \\
\Delta A=\nabla^{2} A & \Delta A_{x} \vec{x}+\Delta A_{y} \vec{y}+\Delta A_{z} \vec{z} & +\left(\Delta A_{\varphi}-\frac{A_{\varphi}}{r^{2}}+\frac{2}{r^{2}} \frac{\partial A_{r}}{\partial \varphi}\right) \vec{\varphi} \\
& +\Delta A_{z} \vec{z}
\end{array}
$$

$$
\left(A_{x} \frac{\partial B_{x}}{\partial x}+A_{y} \frac{\partial B_{x}}{\partial y}+A_{z} \frac{\partial B_{x}}{\partial z}\right) \vec{x} \quad\left(A_{r} \frac{\partial B_{r}}{\partial r}+\frac{A_{\varphi}}{r} \frac{\partial B_{r}}{\partial \varphi}+A_{z} \frac{\partial B_{r}}{\partial z}-\frac{A_{\varphi} B_{\varphi}}{r}\right) \vec{r}
$$

Material derivative $(A \cdot \nabla) B$

$$
\begin{array}{ll}
+\left(A_{x} \frac{\partial B_{y}}{\partial x}+A_{y} \frac{\partial B_{y}}{\partial y}+A_{z} \frac{\partial B_{y}}{\partial z}\right) \vec{y} & +\left(A_{r} \frac{\partial B_{\varphi}}{\partial r}+\frac{A_{\varphi}}{r} \frac{\partial B_{\varphi}}{\partial \varphi}+A_{z} \frac{\partial B_{\varphi}}{\partial z}+\frac{A_{\varphi} B_{\varphi}}{r}\right) \vec{\varphi} \\
+\left(A_{x} \frac{\partial B_{z}}{\partial x}+A_{y} \frac{\partial B_{z}}{\partial y}+A_{z} \frac{\partial B_{z}}{\partial z}\right) \vec{z} & +\left(A_{r} \frac{\partial B_{z}}{\partial r}+\frac{A_{\varphi}}{r} \frac{\partial B_{z}}{\partial \varphi}+A_{z} \frac{\partial B_{z}}{\partial z}\right) \vec{z}
\end{array}
$$




\section{Particle tracing method}

\section{D.1 Introduction}

With the particle tracking method a gas parcel is tracked over a whole cycle in order to get a better inside of the thermodynamic processes occurring close to the cylinder. The path of the parcel is calculated by integrating the velocity in $x$ - and $y$-direction.

\section{D.2 The method}

The simulation provides the whole flow field at 40 instances of each period in the Eulerian description of continuum. To visualize the thermodynamic process, a gas parcel has to be followed. For this reason the description of continuum has to be adapted into the Lagrangian description. In the Lagrangian point of view the path of the gas parcel is chosen such that the velocity is equal to the fluid velocity. The position of the gas parcel one time step later can be estimated as:

$$
x=x_{\text {start }}+\int_{0}^{\Delta t} u(t, x) d t
$$

Or in a differential form:

$$
\frac{d x}{d t}=u(t, x)
$$

In a first approach this can be approximated by an explicit Euler scheme as follows:

$$
x_{n}=x_{s}+\Delta t \cdot u(t, x)
$$

But this does not lead to good results as especially for low frequencies the time steps are too big. Using this method the gas parcels enter the solid and due to interpolation do not exit it anymore.

Two solutions are possible, the first is to export more time steps per period, especially for low frequencies. The second is to use a better approximation, like for example the Runge-Kutta method.

In this paper the forth order Runge-Kutta method is applied. The new position $x_{n}$ is calculated from the start position $x_{s}$ using a weighted average of four increments as follows [wikipedia]:

$$
x_{n}=x_{s}+\frac{1}{6} \Delta t\left(k_{1}+2 k_{2}+2 k_{3}+k_{4}\right)
$$

Where the values $k_{i}$ represent the velocity for the time increment $i$. The $k_{i}$ are given by:

$$
k_{1}=u\left(t_{n}, x_{s}\right)
$$




$$
\begin{gathered}
k_{2}=u\left(t_{n}+\frac{1}{2} \Delta t, x_{s}+\frac{1}{2} \Delta t k_{1}\right) \\
k_{3}=u\left(t_{n}+\frac{1}{2} \Delta t, x_{s}+\frac{1}{2} \Delta t k_{2}\right) \\
k_{4}=u\left(t_{n}+\Delta t, x_{s}+\Delta t k_{3}\right)
\end{gathered}
$$

At the new location the fluid data is exported for post processing. Because the selfimplemented Runge Kutta method was not accurate enough and a $6^{\text {th }}$ order Runge-Kutta was neither, the ode45 solver of MATLAB is used to solve the differential equation (5.5). This gives considerably more accurate results, as the particle then nearly follows one path over different periods.

a)

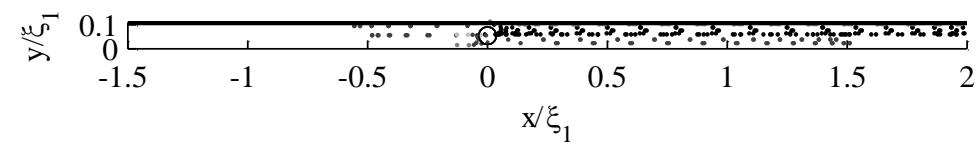

b)

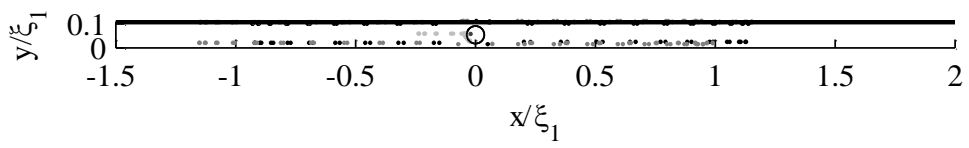

c)

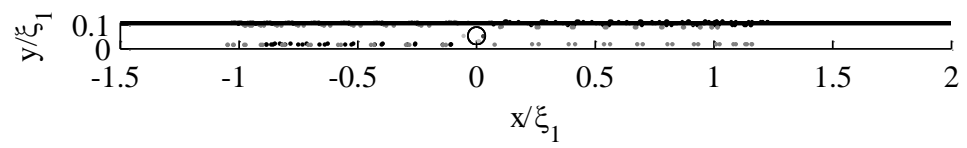

d)

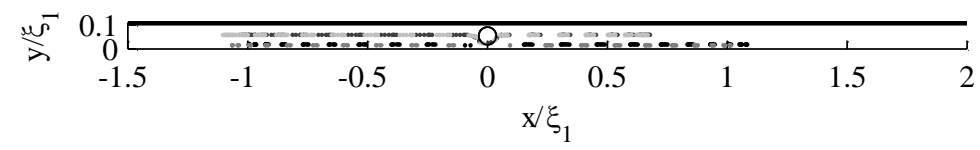

Figure D.1: Particle displacement for different integration methods: a) explicit Euler; b) Runge Kutta Order 4; c) Runge-Kutta Order 6; d) MATLAB ode45 solver (order between 4 and 5 with adaptive time marching) (Sim912)

The difference between the methods is particularly big for simulation with large particle displacement amplitudes. This comes from the high velocities in the narrowing region and the few time steps per period exported. To show the differences between the methods a simulation with a pressure amplitude $p_{1}=100 \mathrm{~Pa}$ and a frequency $f=10 \mathrm{~Hz}$ is chosen. The computed particle displacements are shown in Figure D.1 for the different methods. a) Shows the particle displacement using the explicit Euler method, it can be seen that 
due to the bad approximation the particles enter the cylinder which is unphysical. The method is not accurate enough. b) Shows the results for the fourth order Runge-Kutta method, the particles do not enter anymore the cylinder, but they still don't follow one path and pass through the periodic boundary, which is also not physical as a symmetric flow is expected. c) Shows the results for the $6^{\text {th }}$ order Runge-Kutta method, the results are slightly improved compared to the $4^{\text {th }}$ order, but they are still crossing the symmetry boundary. d) Shows the results for the MATLAB method ode 45 which is a $4^{\text {th }}$ to $5^{\text {th }}$ order method and which uses adaptive time stepping. With this method the results do not enter the cylinder or cross the symmetry boundary conditions, further the particle follows one path over the 4 periods. Thus for post processing of simulation with a large displacement amplitude the MATLAB ode45 function is used.

\section{D.3 Interpolation}

The velocity is only given at discrete places, these are the grid points. Thus an interpolation has to be done to get values inside a mesh cell. The implemented interpolation method works in the following way:

- A Delaunay triangularization of the whole mesh is done (Figure D.2 b))

- The indexes of the triangle containing the point is determined (Figure D.2 c))

- A linear interpolation inside this triangle is done using the MATLAB function scatteredInterpolant() to obtain the flow variables at the desired place (Figure D.2 d)) 
a)

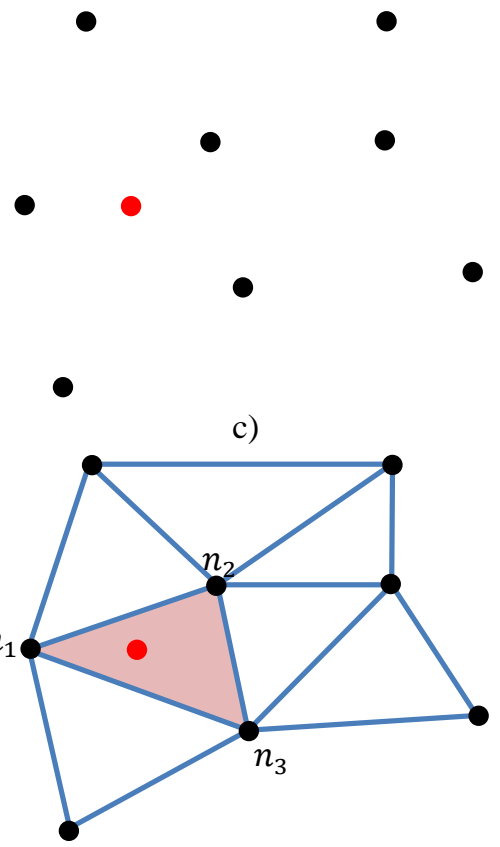

b)

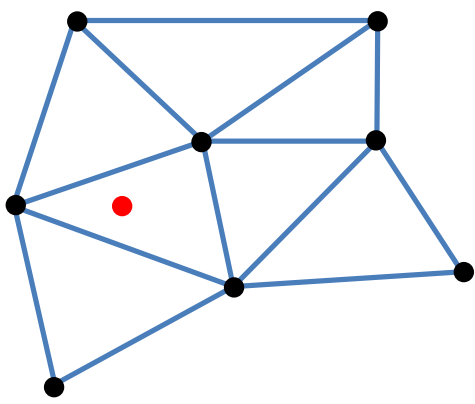

d)

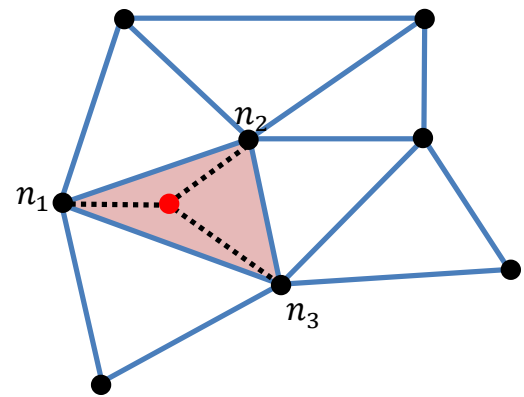

Figure D.2: Visualization of the interpolation method. a) Initial problem, black points represent the mesh points, while the red point represents the point at which the flow data has to be interpolated. b) Delaunay triangularization of the mesh points. c) determination of the indexes of the triangle containing the point for interpolation. d) Interpolation of the flow variables inside of the triangle.

Using the Delaunay triangularization and interpolating only in one triangle reduced the computational time by a factor 100 , compared to directly interpolating on the whole mesh using the MATLAB function scatteredInterpolant(). (This is only valid in our specific case where only a very small number of interpolation have to be done.)

\section{D.4 Correction}

A further correction is implemented, to avoid the gas parcels to leave the domain and leading to an error because the flow variables cannot be interpolated. For this the periodicity in y-direction is used. If a parcel leaves the computational domain on one side, it's $\mathrm{y}$-position is corrected by the domain height $H$ such that it reappears on the other side. 


\section{E Properties of helium}

The properties of helium as they are given in ANSYS Fluent are summarized Table E.1:

Table E.1: Parameters of helium from ANSYS Fluent

\begin{tabular}{cc}
\hline Property & Variable and value \\
\hline Specific heat & $c_{p}=5193 \frac{\mathrm{J}}{\mathrm{kg} \mathrm{K}}$ \\
Thermal conductivity & $k=0.152 \frac{\mathrm{W}}{\mathrm{m} \mathrm{K}}$ \\
Dynamic viscosity & $\mu=1.99 \cdot 10^{-5} \frac{\mathrm{kg}}{\mathrm{m} \mathrm{s}}$ \\
Molecular Weight & $M=4.0026 \frac{\mathrm{kg}}{\mathrm{kmol}}$ \\
\hline
\end{tabular}

Furthermore for the analytical solutions some mean quantities are needed, they are presented in Table E. 2 for a mean pressure of $p_{0}=101325 \mathrm{~Pa}$ and a mean temperature of $T_{0}=300 \mathrm{~K}$ as well as the formula to calculate them:

Table E.2: Derived mean values at $T_{0}=300 \mathrm{~K}$ and a mean pressure of $p_{0}=$ $101325 \mathrm{~Pa}$

\begin{tabular}{ccc}
\hline Property & Formula & Mean value \\
\hline Density & $\rho_{0}=\frac{p_{0}}{\gamma R_{s} T_{0}}$ & $\rho_{0}=0.1626 \frac{\mathrm{kg}}{\mathrm{m}^{3}}$ \\
Speed of sound & $c_{0}=\sqrt{\gamma R_{s} T_{0}}$ & $c_{0}=1019.14 \frac{\mathrm{m}}{\mathrm{s}}$ \\
Specific heat ratio & $\gamma=\frac{c_{p}}{c_{v}}=\frac{1}{1-\frac{R_{s}}{M c_{p}}}$ & $\gamma=1.6667$ \\
\hline
\end{tabular}




\section{E.1 Temperature dependent properties}

Temperature dependent heat conduction and dynamic viscosity are taken as a third order polynomial fit of the properties given in the DeltaEC Manual [7] in the range $T=$ 300..850K:

$$
\begin{aligned}
k=6 & \cdot 10^{-11} \frac{\mathrm{W}}{\mathrm{m} \mathrm{K}^{4}} T^{3}-2 \cdot 10^{-7} \frac{\mathrm{W}}{\mathrm{m} \mathrm{K}^{3}} \cdot T^{2}+0.0005 \frac{\mathrm{W}}{\mathrm{m} \mathrm{K}^{2}} \cdot T \\
& +0.0313 \frac{\mathrm{W}}{\mathrm{mK}} \\
\mu=9 & \cdot 10^{-15} \frac{\mathrm{Pa} \mathrm{s}}{\mathrm{K}^{3}} \cdot T^{3}-3 \cdot 10^{-11} \frac{\mathrm{Pa} \mathrm{s}}{\mathrm{K}^{2}} \cdot T^{2}+6 \cdot 10^{-8} \frac{\mathrm{Pa} \mathrm{s}}{\mathrm{K}} \cdot T \\
& +5 \cdot 10^{-6} \mathrm{~Pa} \mathrm{~s}
\end{aligned}
$$




\section{F Shaking resonator - change in coordi- nate system}

In order to reduce the calculation costs and to avoid a moving mesh, a change in coordinate system is done. Instead of carrying out the simulation in the global system G, a local coordinate system on the resonator $\mathrm{L}$ is defined:

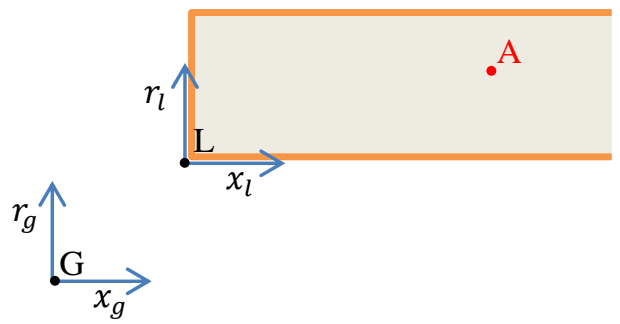

Figure F.1: $\quad$ Definition of the global $(\mathrm{G})$ and local (L) coordinate systems

The variables related to the local and the global coordinate system are indexed with an 1 and a $g$ respectively. In the global coordinate system the position, the velocity and the acceleration of the fluid related to a point $\mathrm{A}$ can be written as:

$$
\begin{aligned}
\vec{r}_{g} & =\vec{r}_{G L}+\vec{r}_{l} \\
\vec{v}_{g} & =\vec{v}_{G L}+\vec{v}_{l} \\
\vec{a}_{g} & =\vec{a}_{G L}+\vec{a}_{l}
\end{aligned}
$$

The velocity of the local coordinate system in the global one is denominated $\vec{u}_{w}$ and can be expressed as:

$$
\vec{u}_{w}=\left(\begin{array}{c}
u_{w} \\
0
\end{array}\right)=\vec{v}_{G L}
$$

\section{F.1 Continuity equation}

To derive the equations in the local coordinate system the Lagrangian point of view with respect to the resonator is adopted. For a general vector function $F$ the material derivative can be written as:

$$
\frac{\mathrm{D} F}{\mathrm{D} t}=\frac{\delta F}{\delta t}+\left(\vec{u}_{w} \cdot \nabla\right) F
$$

With $\vec{u}_{w}$ the velocity of the vibrating tube. This can be applied on the flow variables. The change in density in the Lagrangian point of view can be written as:

$$
\frac{\mathrm{D} \rho}{\mathrm{D} t}=\frac{\delta \rho}{\delta t}+\left(\vec{u}_{w} \cdot \nabla\right) \rho=\frac{\delta \rho}{\delta t}+u_{w} \frac{\delta \rho}{\delta x}
$$


The local time derivative of the density can be replaced due to the continuity equation:

$$
\frac{\mathrm{D} \rho}{\mathrm{D} t}=-\frac{\delta\left(\rho u_{g}\right)}{\delta x}-\frac{\delta\left(\rho v_{g}\right)}{\delta y}+u_{w} \frac{\delta \rho}{\delta x}
$$

And according to (F.1) the velocity can be replaced:

$$
\frac{\mathrm{D} \rho}{\mathrm{D} t}=-\frac{\delta\left(\rho u_{l}\right)}{\delta x}-\frac{\delta\left(\rho u_{w}\right)}{\delta x}-\frac{\delta\left(\rho v_{g}\right)}{\delta y}+u_{w} \frac{\delta \rho}{\delta x}
$$

Applying the product rule leads to:

$$
\frac{\mathrm{D} \rho}{\mathrm{D} t}=-\frac{\delta\left(\rho u_{l}\right)}{\delta x}-\rho \frac{\delta u_{w}}{\delta x}-u_{w} \frac{\delta \rho}{\delta x}-\frac{\delta\left(\rho v_{g}\right)}{\delta y}+u_{w} \frac{\delta \rho}{\delta x}
$$

As the velocity of the resonator $u_{w}$ does not depend on $x$, the continuity equation can finally be written as:

$$
\frac{\mathrm{D} \rho}{\mathrm{D} t}=-\frac{\delta\left(\rho u_{l}\right)}{\delta x}-\frac{\delta\left(\rho v_{l}\right)}{\delta y}
$$

\section{F.2 Momentum equation}

The substantial derivative of the momentum can be written as:

$$
\frac{\mathrm{D}}{\mathrm{D} t}\left(\rho \vec{v}_{g}\right)=\frac{\delta}{\delta t}\left(\rho \vec{v}_{g}\right)+\left(\vec{u}_{w} \cdot \nabla\right)\left(\rho \vec{v}_{g}\right)
$$

Or for the momentum in $\mathrm{x}$-direction:

$$
\frac{\mathrm{D}}{\mathrm{D} t}\left(\rho u_{g}\right)=\frac{\delta}{\delta t}\left(\rho u_{g}\right)+\left(u_{w} \cdot \nabla\right)\left(\rho \vec{v}_{g}\right)
$$

The left side can be rewritten as:

$$
\begin{gathered}
\frac{\mathrm{D}}{\mathrm{D} t}\left(\rho u_{g}\right)=\frac{\mathrm{D}}{\mathrm{D} t}\left(\rho\left(u_{l}+u_{w}\right)\right)=\frac{\mathrm{D}}{\mathrm{D} t}\left(\rho u_{l}\right)+\frac{\mathrm{D}}{\mathrm{D} t}\left(\rho u_{w}\right) \\
=\frac{\mathrm{D}}{\mathrm{D} t}\left(\rho u_{l}\right)+\rho \frac{\mathrm{D} u_{w}}{\mathrm{D} t}+u_{w} \frac{\mathrm{D} \rho}{\mathrm{D} t}
\end{gathered}
$$

While the momentum equation can be rewritten according to (F.1) in the following way:

$$
\begin{aligned}
\frac{\delta}{\delta t}\left(\rho u_{g}\right)=- & {\left[\frac{\delta}{\delta x}\left(\rho u_{g}^{2}+p-\tau_{x x}\right)+\frac{\delta}{\delta y}\left(\rho u_{g} v_{g}-\tau_{x y}\right)\right] } \\
=- & {\left[\frac{\delta}{\delta x}\left(\rho u_{l}^{2}+p-\tau_{x x}\right)+\frac{\delta}{\delta y}\left(\rho u_{l} v_{l}-\tau_{x y}\right)+u_{w} \frac{\delta\left(\rho u_{l}\right)}{\delta x}\right.} \\
& \left.\quad+u_{w} \frac{\delta(\rho v)}{\delta x}+u_{w} \frac{\delta\left(\rho u_{l}\right)}{\delta x}+\frac{u_{w}^{2} \delta \rho}{\delta x}\right]
\end{aligned}
$$

The third and the forth term on the right hand side can be replaced with the continuity equation (F.8) and the last two terms are rewritten according to the product rule: 


$$
\begin{gathered}
\frac{\delta}{\delta t}\left(\rho u_{g}\right)=-\left[\frac{\delta}{\delta x}\left(\rho u_{l}^{2}+p-\tau_{x x}\right)+\frac{\delta}{\delta y}\left(\rho u_{l} v_{l}-\tau_{x y}\right)+u_{w} \frac{\mathrm{D} \rho}{\mathrm{D} t}\right. \\
\left.+u_{w} \frac{\delta\left(\rho\left(u_{l}+u_{w}\right)\right.}{\delta x}\right]
\end{gathered}
$$

Filling in (F.13) and (F.11) in (F.10) leads to:

$$
\frac{\mathrm{D}}{\mathrm{D} t}\left(\rho u_{l}\right)=-\left[\frac{\delta}{\delta x}\left(\rho u_{l}^{2}+p-\tau_{x x}\right)+\frac{\delta}{\delta y}\left(\rho u_{l} v_{l}-\tau_{x y}\right)\right]-\rho \frac{\mathrm{D} u_{w}}{\mathrm{D} t}
$$

The same can be done with the momentum equation in y-direction:

$$
\frac{\mathrm{D}}{\mathrm{D} t}\left(\rho v_{l}\right)=-\left[\frac{\delta}{\delta x}\left(\rho v_{l} u_{l}+p-\tau_{x y}\right)+\frac{\delta}{\delta y}\left(\rho v_{l}^{2}-\tau_{y y}\right)\right]
$$

The right hand sides of the equations (F.8), (F.14) and (F.15) are the Navier-Stokes equation for a fluid in the local coordinate system, with an additional volume force representing the acceleration of the resonator.

\section{F.3 Energy Equation}

In the same way the energy equation in the local coordinate system is derived. The following source term has to be added:

$$
S_{h}=-\rho u_{l} \frac{\mathrm{D} u_{w}}{\mathrm{D} t}
$$

By implementing these terms in Fluent, the experiment can be carried out without any moving mesh. The variables solved for are given in the local coordinate system, to get the variables according to the global system a transformation back according to equation (F.1) has to be done. 


\section{G Point definition for shaking resonator}

The results are monitored at the following points. The lines BB and DD are the lines over the radius at the points $\mathrm{D}$ and $\mathrm{B}$.

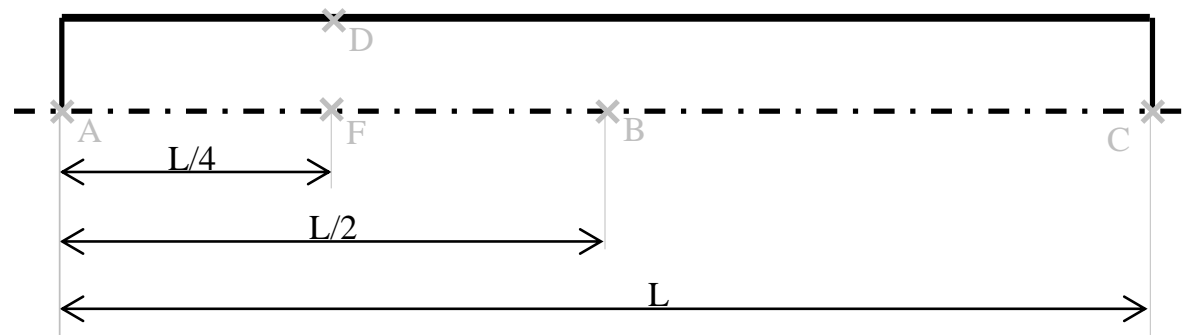

Figure G.1: Definition of the geometrical location in the vibrating resonator 


\section{H Solving the heat equation with MATLAB}

The heat equation can be solved for the temperature if the pressure and the velocity are known. The procedure is similar to the one of Matveev et al. [76] and Berson et al. [92]. The heat equation as is solved numerically with the pdepe-function of MATLAB. The pdepe-function solves initial-boundary problems for parabolic PDEs in one dimension.

The pdepe-function can solve a differential equation, which can be written in the following form:

$$
c \frac{\partial u}{\partial t}=\frac{\partial}{\partial x}(f)+s
$$

For this reason the heat equation is rewritten to fit the input of the pdepe-function:

$$
\begin{aligned}
\frac{\gamma}{\gamma-1} \frac{p}{T} \frac{1}{k} \frac{\partial T}{\partial t}= & \frac{\partial}{\partial x}\left(\frac{\partial T}{\partial x}\right)+\frac{1}{k} \frac{d p}{d t}+\frac{\gamma}{\gamma-1} \frac{1}{k} \frac{p}{T} K\left(T_{H X}-T\right) \\
& -\frac{\gamma}{\gamma-1} \frac{p}{T} \frac{1}{k} u \frac{\partial T}{\partial x}
\end{aligned}
$$

with:

$$
\begin{gathered}
c=\frac{\gamma}{\gamma-1} \frac{p}{T} \frac{1}{k} \\
f=\frac{\partial T}{\partial x} \\
s=\frac{1}{k} \frac{d p}{d t}+\frac{\gamma}{\gamma-1} \frac{1}{k} \frac{p}{T} K\left(T_{H X}-T\right)-\frac{\gamma}{\gamma-1} \frac{p}{T} \frac{1}{k} u \frac{\partial T}{\partial x}
\end{gathered}
$$

The total domain length $L$ is chosen the same as in the simulations. Within the heat exchanger the heat transfer coefficient is set to $K \neq 0$, while outisde the heat exchanger $K=0$. The pressure and the velocity are uniform in the domain and change in time according to:

$$
\begin{gathered}
p(t)=p_{0}+p_{1} \sin (\omega t) \\
u(t)=\frac{p_{1}}{\rho_{0} c_{0}} \sin (\omega t+\varphi)
\end{gathered}
$$

The temperature boundary conditions at the left and the right of the domain are calculated from the pressure assuming an adiabatic process. When applicable the simulations are initialized with a constant temperature. In the thermal buffer tube simulations the initialization of the temperature field needs more attention as a large temperature gradient is imposed in the thermal buffer tube that changes the fluid properties. The initial temperature field is obtained by solving the steady state heat equation:

$$
\frac{\partial}{\partial x}\left(k \frac{\partial T}{\partial x}\right)=-K \frac{\gamma}{\gamma-1} \frac{p}{T}\left(T_{H X}-T\right)
$$


with the $b v p 5 c$-function that solves boundary value problems. The initial temperature profile in the case of an adiabatic wall boundary condition is shown in Figure H.1. The fluid parameters are taken the same as in the corresponding simulation and the $\mathrm{K}$ value is adapted to fit the simulations.

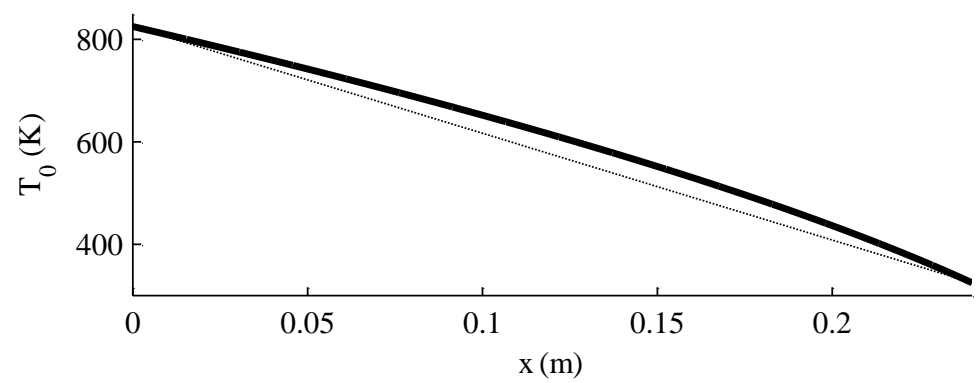

Figure H.1: Initial temperature profile in the thermal buffer tube assuming an adiabatic wall boundary condition. Dashed line indicates a linear profile. 


\section{Influence of errors on calculation of thermoacoustic functions}

In this chapter the influence of errors on the estimation of the thermoacoustic function is investigated. The errors can come from the phase and amplitude calculation, which are necessary to transform the experimental data from the time domain to the frequency domain, used for post processing. To check the influence of errors, the theoretical model is run, but before applying the post processing to calculate the thermoacoustic functions, the pressure or the velocity and the right end of the domain (see Figure 5.36: Position 12) are modified in order to model errors. The amplitude and the phase are changed independently to determine the influence of each.
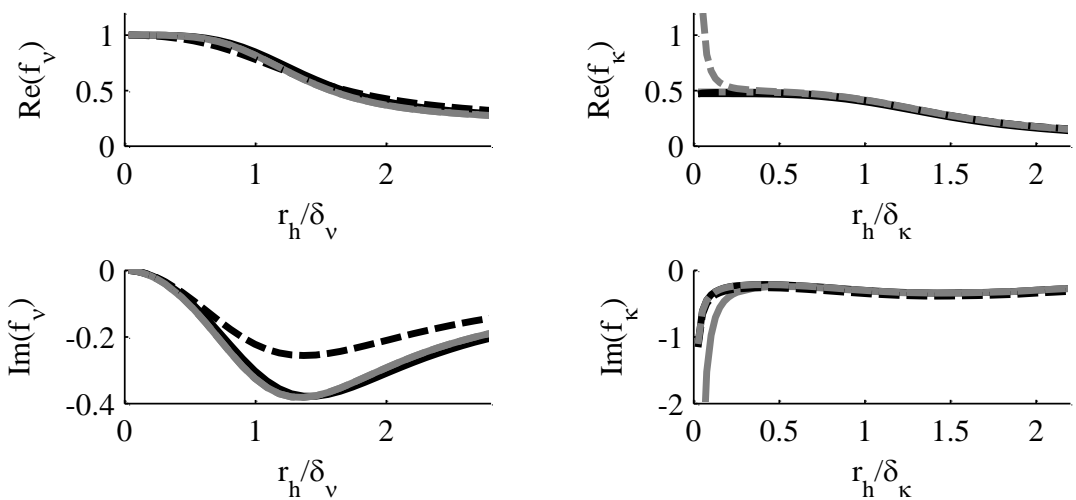

a)

b)

$$
\begin{aligned}
& -\mathrm{p}_{\mathrm{e}}:|1|, \phi=0-\mathrm{u}_{\mathrm{e}}:|1|, \phi=0 \\
& \mathrm{p}_{\mathrm{e}}:|1.01|, \phi=0-\mathrm{u}_{\mathrm{e}}:|1|, \phi=0 \\
& \mathrm{p}_{\mathrm{e}}:|1|, \phi=0-\mathrm{u}_{\mathrm{e}}:|1.0001|, \phi=0
\end{aligned}
$$

$$
\begin{aligned}
& \text {-ேーー } \mathrm{p}_{\mathrm{e}}:|1|, \phi=0.01-\mathrm{u}_{\mathrm{e}}:|1|, \phi=0 \\
& \text {-ேーー } \mathrm{p}_{\mathrm{e}}:|1|, \phi=0-\mathrm{u}_{\mathrm{e}}:|1|, \phi=1 \mathrm{e}-05
\end{aligned}
$$

Figure I.1: Influence of small deviation of the phase and the amplitude of the pressure and the velocity on the calculation of the thermal a) and viscous b) thermoacoustic function. (reference case in red)

First the influence of the phasing on the calculation of the thermoacoustic function is discussed. From Figure I.1 a) it can be seen, that even very small errors in the phasing of the velocity can have a large influence on the real part of the thermal thermoacoustic function. This is the trend which can also be seen in the numerical experiments, which indicates that the calculation of the velocity phasing has to be very accurate. The phasing of the velocity has no further influence on other parts of the thermoacoustic functions. 
The phasing of the pressure has a much less important effect, the error has to be three orders of magnitudes higher than for the velocity before a deviation can be seen. With this high deviation all values of the thermoacoustic functions are affected. As the effect of velocity phasing errors occurs for much smaller values, the velocity errors define the necessary accuracy of the phase calculations.

Next to the influence of errors in the phasing, the influence of amplitude errors have to be investigated. A $0.01 \%$ deviation of the amplitude of the velocity has a large influence on the imaginary part of the thermoacoustic function, thus the thermal resistance is estimated inaccurately. The other parts of the thermoacoustic function are not affected. A $1 \%$ deviation of the pressure amplitude has a small influence on the real part of the thermoacoustic function and a larger influence on the viscous thermoacoustic function. This means that if the pressure amplitude is estimated to high, the viscous contact between the gas and the plates are overestimated. But again the errors on the pressure amplitude have to be two orders of magnitude higher than the velocity amplitude errors.

With this we can conclude that the correct measurement of the velocity is crucial for accurate estimation of the thermoacoustic functions.

The reason why errors are most important for the thermal thermoacoustic functions can be seen from equation (I.1) and (I.2):

$$
\begin{gathered}
d p_{1}=-I \frac{\omega \rho_{0}}{1-f_{v}} \frac{U_{1}}{A} d x \\
d U_{1}=-\frac{I \omega A}{\gamma p_{m}}\left(1+(\gamma-1) f_{\kappa}\right) p_{1} d x
\end{gathered}
$$

With decreasing frequency the changes in pressure and velocity over the investigated regenerator length decrease due the angular frequency $\omega$. For the pressure changes this effect is attenuated by the $1 /\left(1-f_{v}\right)$ term, which goes to infinity towards low frequencies. In general this means this means that the thermoacoustic functions are calculate from smaller and smaller changes over the pore, which leads to errors. Especially on the thermal thermoacoustic function as the changes over the regenerator length are especially small for the volume flow rate.

One way of solving this problem would be to increase the investigated regenerator length such that the changes over the regenerator length get larger, but this results in an increase of the calculation time. Another way would be to vary the fluid properties, for example the mean pressure or density. Finally a more accurate method for the calculation of the phase and the amplitude of the pressure and velocity could be implemented in order to reduce the error.

\section{I.1 Rounding errors}

To check the influence of rounding errors, the theoretical model is run, but before launching the post processing to calculate the thermoacoustic functions, the pressure or 
the velocity and the right end of the domain (see Figure 5.36: Position 12) are rounded at three different precisions, to get an idea of the number of necessary significant digits. Because the deviations were biggest for the thermal thermoacoustic function, only this one is presented in the following. From the results in Figure I.2 one can conclude that in order to have accurate results at low frequencies the variables have to be accurate be at least six significant digits.

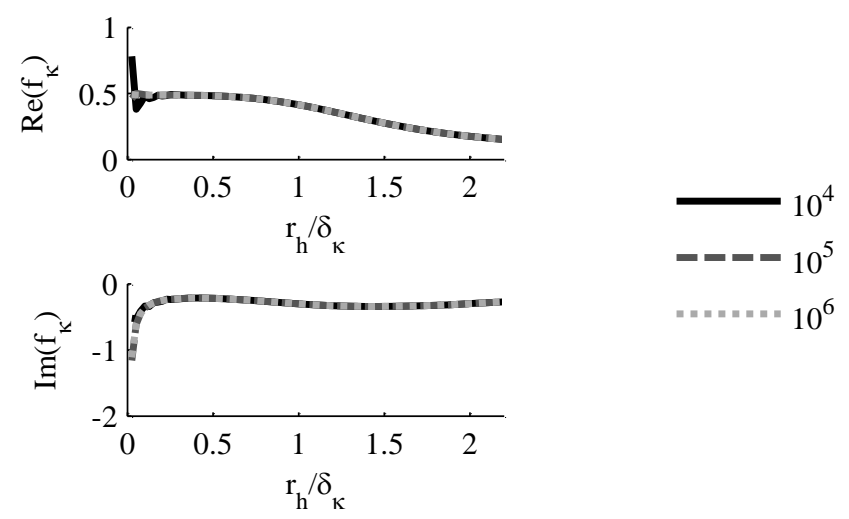

Figure I.2: Influence of rounding errors in the pressure and the velocity on the calculation of the thermal thermoacoustic function.

\section{I.2 Errors on acoustic constants}

Due to the discreet grid the wave propagation constant and the impedance are not equal to their theoretical value. For this reason the error sensibility is tested here. Only for large errors, as shown here, a deviation can be seen. Again errors have a smaller influence on the viscous thermoacoustic function than on the thermal thermoacoustic function, but in general the behavior of both is the same. The real part of the thermoacoustic function is influenced by the amplitude of the wave propagation constant and the acoustic impedance, while phase errors influence the imaginary part. But none of these deviations can explain the difference between the model and the simulations. 

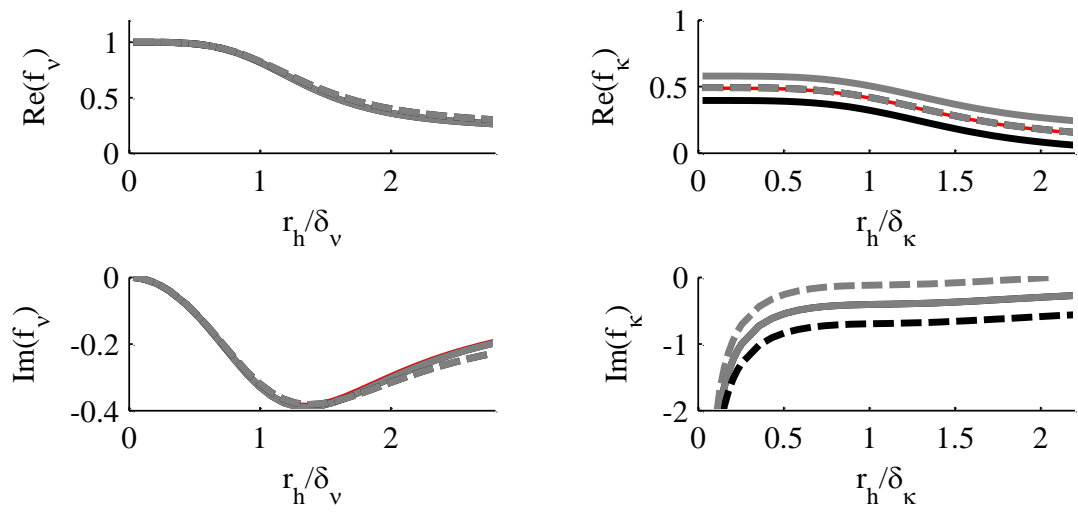

a)

b)

$$
\begin{aligned}
& \mathrm{k}_{\mathrm{e}}:|1|, \phi=0-\mathrm{Z}_{\mathrm{e}}:|1|, \phi=0 \\
& \mathrm{k}_{\mathrm{e}}:|1.01|, \phi=0-\mathrm{Z}_{\mathrm{e}}:|1|, \phi=0 \\
& \mathrm{k}_{\mathrm{e}}:|1|, \phi=0-\mathrm{Z}_{\mathrm{e}}:|1.01|, \phi=0
\end{aligned}
$$

-ーー $\mathrm{k}_{\mathrm{e}}:|1|, \phi=0.01-\mathrm{Z}_{\mathrm{e}}:|1|, \phi=0$$$
-\mathrm{k}_{\mathrm{e}}:|1|, \phi=0-\mathrm{Z}_{\mathrm{e}}:|1|, \phi=0.01
$$

Figure I.3: Influence of small deviations of the phase and the amplitude of the wave propagation constant and the acoustic impedance on the calculation of the thermal a) and viscous b) thermoacoustic function. (reference case in red) 


\section{$J$ Scaling of thermal thermoacoustic func- tions}

In Chapter 5.3 it is noted that for low frequencies or low Lautrec numbers respectively the values of the one-dimensional model converges to a constant value, which is clearly smaller than one. In this chapter it is shown, why the thermal thermoacoustic function does not go to one for low frequencies. The model introduced in Chapter 5.3.1.2 is used in order to simulate the reduced geometry given in Figure J.1. The thermoacoustic functions of the whole geometry are calculated for three different distances $x_{c}$ between the parallel plates that model the cylinders.

The results are shown in Figure J.2 a) and indeed the real part of the thermoacoustic function does not go to one for low frequencies. The reason is that in the reduced model the heat transfer does not occur over the whole axial length of the regenerator, but only over the length of the cylinders. Therefore a scaling factor $r_{\text {scale }}$ is introduced that takes this into account. The scaling factor is defined as the length of the regenerator, consisting of $n$ screens divided by the length over which heat transfer occurs:

$$
r_{\text {scale }}=\frac{n x_{b}+n x_{c}}{n x_{b}}=1+\frac{x_{c}}{x_{b}}
$$

Figure J.2 b) shows the scaled thermal thermoacoustic function and indeed the real part goes to one for low frequencies. It has to be remarked that in real regenerators at low frequencies the heat conduction in axial direction gets increasingly important, such that this scaling is not applicable there, as conduction in the axial direction was neglected in this derivation.

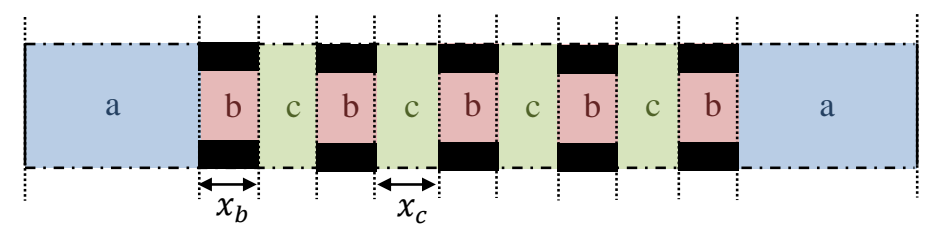

Figure J.1: Different parts of the acoustic model: a adiabatic part; b part with flow between parallel plates; $\mathrm{c}$ part between the parallel plates. 

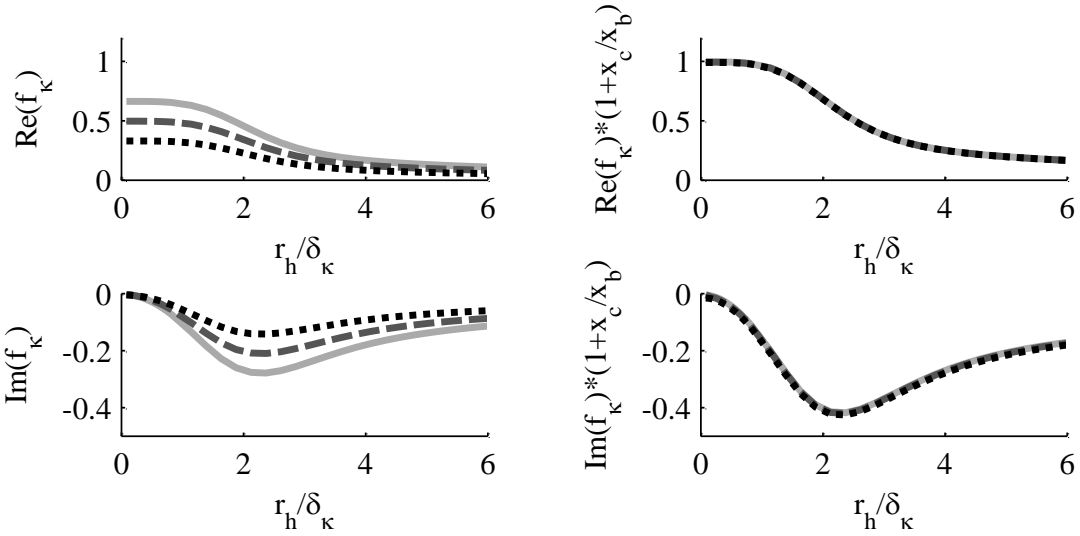

a)

b)

Figure J.2: a) unscaled und b) scaled thermal thermoacoustic function from reduced model for three different distances between the cylinders: $x_{c}=0.5 \cdot x_{b}, 1$. $x_{b}$ and $2 \cdot x_{b}$, in light gray, dark gray and black respectively. 


\section{K Thermal buffer tube boundary condi- tions}

In this chapter a motivation for the choice of the acoustic boundary conditions of the thermal buffer tube study in Chapter 5.1 is given. In all cases the right boundary condition is set to a non-reflecting pressure boundary condition and the left boundary condition is varied.

From all different wall boundary conditions simulated, the case with adiabatic slip wall boundary conditions is the most demanding as in this case the least physical dissipation occurs and numerical errors are most visible. For this reason the investigation of the acoustic boundary condition will only be done for a case with adiabatic slip wall boundary conditions. This wall boundary condition has also the advantage that the domain can be reduced to a one-dimensional problem, reducing the needed computational resources. This is confirmed with a preliminary study that compares a one-dimensional case with a two-dimensional case. Both overlay each other. A motivation for the choice of the left boundary condition is given in the following.

\section{K.1 Non-reflecting boundary conditions}

First, the results are presented when a non-reflecting boundary condition as introduced in Chapter 4.5.1 is imposed at the left of the thermal buffer tube. The pressure the velocity and the temperature at the center of the thermal buffer tube are given in Figure K.1, Figure K.2 and Figure K.3, respectively. The figures show that the pressure and the velocity drift towards a lower value and that the amplitude is reduced during the simulations. The negative mean velocity introduces convection, such that gas with the temperature of the ambient heat exchanger is convected inside of the domain. As can be seen in Figure K.3 this leads to a reduced temperature inside of the thermal buffer tube. This streaming is not physical, but introduced by the acoustic boundary conditions. For this reason this boundary condition cannot be used to investigate the thermal buffer tube. An attempt was done to implement a non-reflecting boundary condition at the left by imposing the velocity or the mass-flux such that the streaming can be reduced, but both were not successful and the corresponding test cases crashed after several time steps. For this reason it was chosen to impose a fully reflective boundary condition at the left of the domain. The choice of the boundary condition will be shown in the following chapter. 


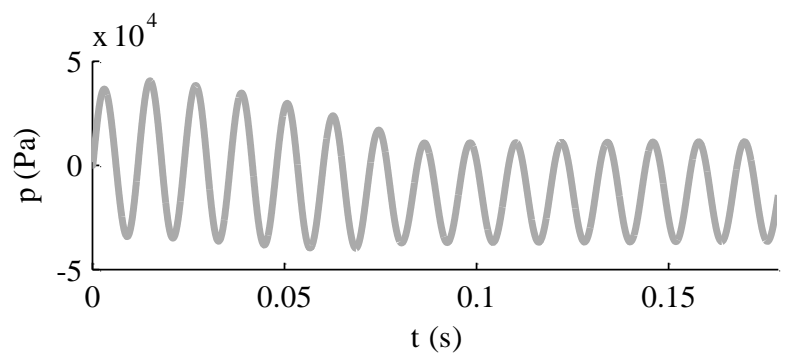

Figure K.1: Pressure at the center of the thermal buffer tube for a non-reflecting boundary condition at the left of the domain over time.

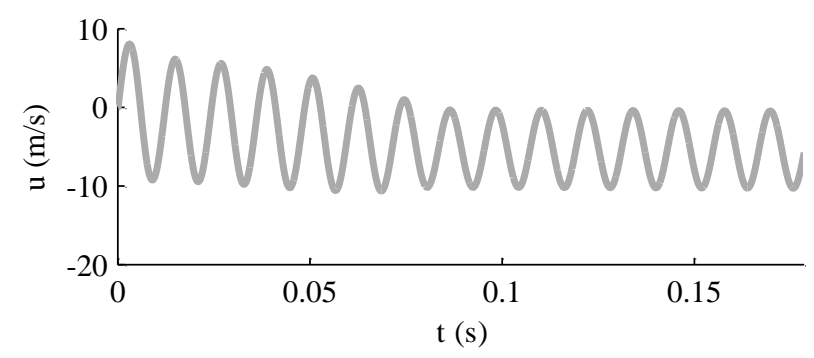

Figure K.2: Velocity at the center of the thermal buffer tube for a non-reflecting boundary condition at the left of the domain over time.

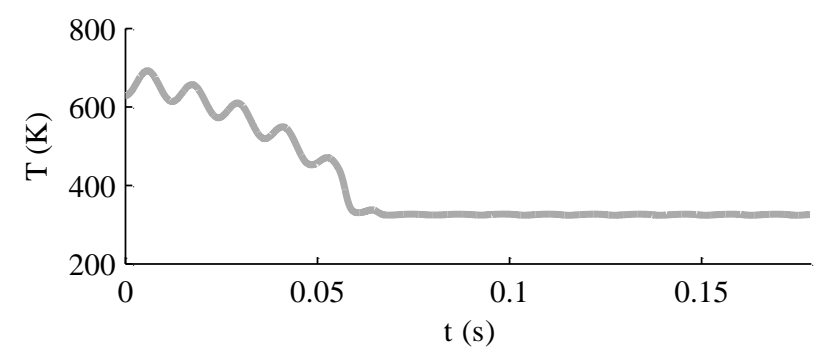

Figure K.3: Temperature at the center of the thermal buffer tube for a non-reflecting boundary condition at the left of the domain over time.

\section{K.2 Reflecting boundary condition}

In this chapter the non-reflecting character of the left boundary condition is switched off. This means that a defined time dependent harmonic value is imposed at the left boundary, while the right boundary is set to a non-reflecting pressure boundary condition. The wave at the left is introduced by using three types of boundary conditions: a pressure inlet, velocity inlet and a mass flux inlet.

Figure K.4 shows that using the mass flux inlet and the velocity inlet introduce a large drifting in pressure, while no drift occurs for the pressure inlet. Figure K.5 shows the velocity over time at the center of the thermal buffer tube for the three different boundary 
conditions. It can be seen, that for the mass flux and velocity boundary condition no drift occurs and the values oscillate as expected around zero. But using a pressure boundary condition introduces a non-zero mean flow. This can also be seen in Figure K.6, where the temperature in the thermal buffer tube falls. Heat is thus convected from the cold heat exchanger to the hot heat exchanger. Using a velocity inlet the temperature also increases slightly over time, which indicates a mass flux from the hot heat exchanger to the ambient heat exchanger.

The same experiment is also performed without applying a temperature gradient over the domain, but setting the domain temperature constant to either of the heat exchanger temperatures. For both cases and all boundary condition no drift occurs and the pressure and velocity values oscillate around zero. From this it can be concluded that the drifting originates from the temperature gradient inside of the thermal buffer tube. One possible reason could be that the speed of sound is not estimated correctly. For this reason the boundary treatment is adapted in the following chapter, such that the position of the monitor point is recalculated every time step.

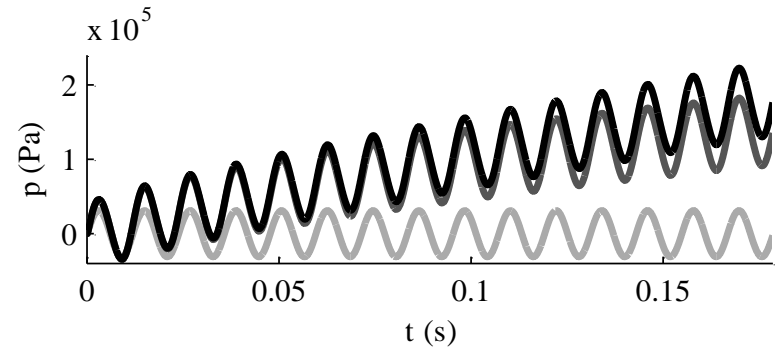

Figure K.4: Pressure at the center of the thermal buffer tube over time. Results for the three different reflecting boundary condition at the left of the domain: pressure inlet, mass flux inlet and velocity inlet, in light gray, dark gray and black respectively.

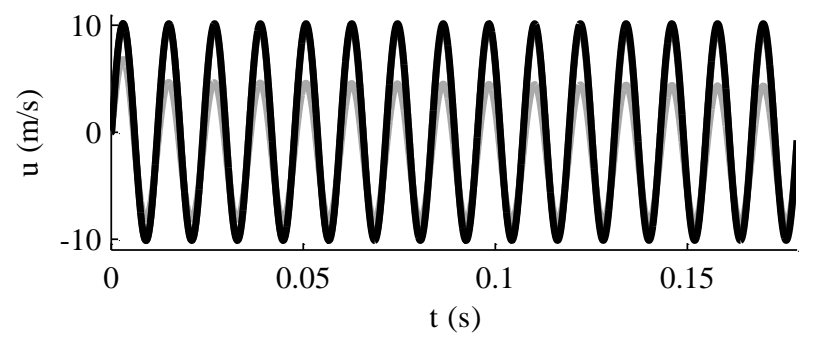

Figure K.5: Velocity at the center of the thermal buffer tube over time. Results for the three different reflecting boundary condition at the left of the domain: pressure inlet, mass flux inlet and velocity inlet, in light gray, dark gray and black respectively. 


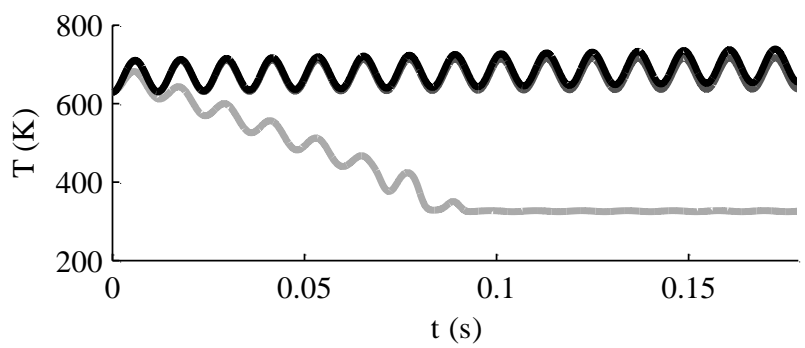

Figure K.6: Temperature at the center of the thermal buffer tube over time. Results for the three different reflecting boundary condition at the left of the domain: pressure inlet, mass flux inlet and velocity inlet, in light gray, dark gray and black respectively.

\section{K.3 Variable monitor point}

As was shown in the previous chapter, the drifting of the physical quantities is probably due to an incorrect estimation of the speed of sound and thus of the location of the monitor point for the acoustic boundary condition at the right. For this reason the boundary treatment for the right boundary condition is adapted in this chapter, such that the position of the monitor point is recalculated every time step from the speed of sound at that moment. This additional calculation is computationally expensive and did not show any improvement in case of a constant mean temperature (see Chapter 4.2.2.3). For this reason it is not used for the cases with constant temperature, unlike the case here.

The three different inlet types at the left are differentiated in the following. Figure K.7 shows the pressure over time for the three boundary conditions. It can be seen that for the pressure boundary condition no drifting or shifting occurs. For the velocity boundary condition the pressure drifts away, over the whole simulation time. This is not the case for the mass flux boundary condition where the mean value of the pressure drifts during the first periods but then converges to a constant value. For all boundary conditions the axial velocity does not drift, as is shown in Figure K.8. But for the pressure boundary condition a small lower order oscillation occurs. This oscillation influences the temperature, as heat is convected. This can be seen in Figure K.9 as the mean value of the temperature oscillates for the pressure boundary condition. The mass flux boundary condition performs the best, as it only drifts in a really small amount and the reflection coefficient at the right side stays below $0.5 \%$.

Concluding it can be said that adapting the position of the monitor point at each time step improves the simulation results. The drifting might be caused by the temperature dependency of the speed of sound. In general when a mass flux boundary condition is imposed at the left the best results are achieved. For this reason this boundary condition is applied in the results given in Chapter 5.1. 


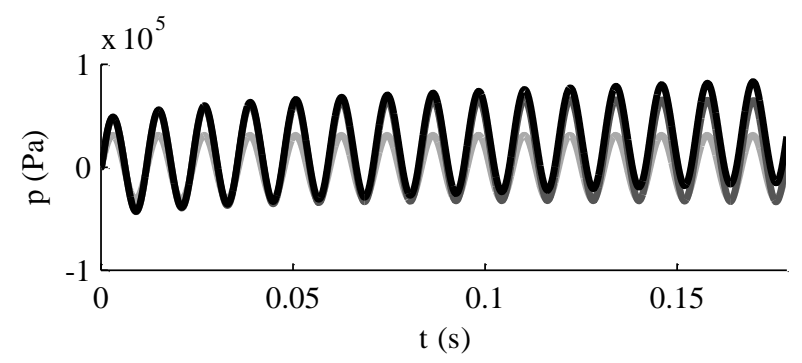

Figure K.7: Pressure at the center of the thermal buffer tube over time. Results for the three different reflecting boundary condition at the left of the domain: pressure inlet, mass flux inlet and velocity inlet, in light gray, dark gray and black respectively.

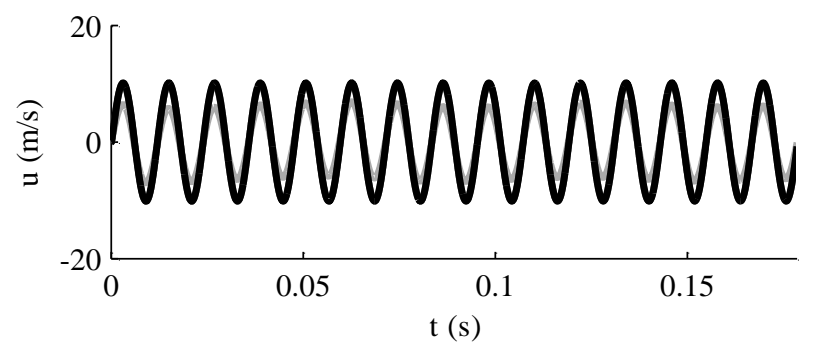

Figure K.8: Velocity at the center of the thermal buffer tube over time. Results for the three different reflecting boundary condition at the left of the domain: pressure inlet, mass flux inlet and velocity inlet, in light gray, dark gray and black respectively.

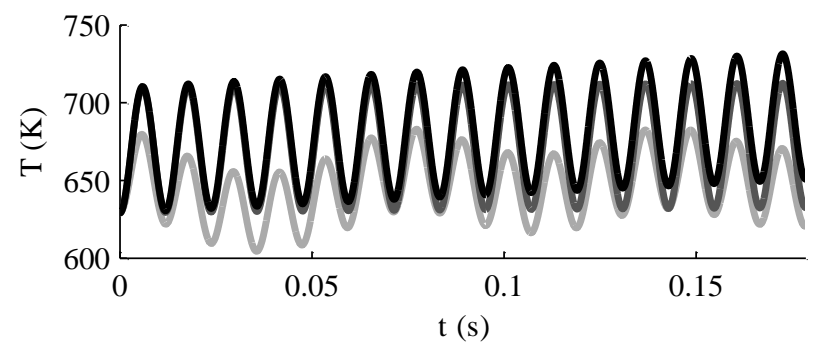

Figure K.9: Temperature at the center of the thermal buffer tube over time. Results for the three different reflecting boundary condition at the left of the domain: pressure inlet, mass flux inlet and velocity inlet, in light gray, dark gray and black respectively. 


\section{Thermal buffer tube mesh refinement}

In this chapter the mesh refinement study done for the thermal buffer tube investigated in Chapter 5.1 is shortly summarized. First in Table L. 1 the properties of the different meshes are given. These meshes are then compared in order to show that the selected mesh is converged. In Figure L.1 the mean temperature profile is given for the case with an adiabatic slip wall boundary condition. This is the only case shown here, as this is the most demanding case, as the least physical dissipation occurs and numerical errors are most visible. The results from the "Fine" mesh deviates from the other meshes. The reason is probably the refinement in the axial direction and the reduced number of elements in the center of the thermal buffer tube. For all other meshes, the mean temperature profiles converge with increasing number of elements towards the finest mesh. The differences between the two finest meshes are hardly visible, it can thus be concluded that the mesh is converged. These parameters are thus used in Chapter 5.1.

Table L.1: Definition of the different meshes investigated.

\begin{tabular}{|c|c|c|c|}
\hline Mesh type & $\begin{array}{l}\text { Elements in } \\
y \text {-direction }\end{array}$ & $\begin{array}{l}\text { Elements in } \\
x \text {-direction }\end{array}$ & Name \\
\hline $\begin{array}{l}\text { Refinement in radial direction } \\
\text { Uniform in axial direction }\end{array}$ & 30 & 101 & "Rough" \\
\hline $\begin{array}{l}\text { Refinement in radial direction } \\
\text { Uniform in axial direction }\end{array}$ & 45 & 151 & $\begin{array}{l}\text { "Medium } \\
\text { rough" }\end{array}$ \\
\hline $\begin{array}{l}\text { Refinement in radial direction } \\
\text { Refinement in towards the } \\
\text { boundaries }\end{array}$ & 60 & 177 & "Fine" \\
\hline $\begin{array}{l}\text { Refinement in radial direction } \\
\text { Uniform in axial direction }\end{array}$ & 60 & 201 & $\begin{array}{c}\text { "Fine } \\
\text { constant" }\end{array}$ \\
\hline $\begin{array}{l}\text { Refinement in radial direction } \\
\text { Uniform in axial direction }\end{array}$ & 60 & 301 & $\begin{array}{l}\text { "Finer } \\
\text { axial" }\end{array}$ \\
\hline
\end{tabular}




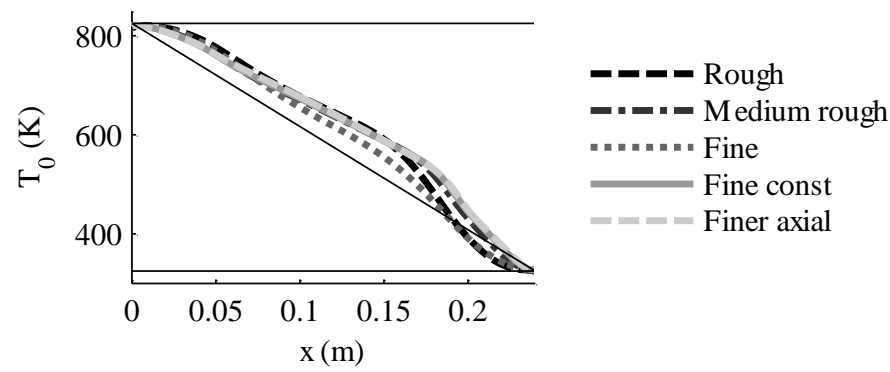

Figure L.1: Mean temperature profile inside of the thermal buffer tube for different spatial discretizations and for adiabatic slip wall boundary condition. 


\section{Publications}

Bühler, S.; Wilcox, D.; Oosterhuis, J. P. \& Van der Meer, T. H.

Calculation of thermoacoustic functions with computational fluid dynamics

Proceedings of Meetings on Acoustics, Vol. 19, ASA, 2013

Bühler, S.; Wilcox, D.; Oosterhuis, J. P. \& Van der Meer, T. H.

Thermal entrance effects in a thermoacoustic stacked screen regenerator

Proceedings of the 15th International Heat Transfer Conference, 2014, 1-9

Bühler, S.; Wilcox, D.; Oosterhuis, J. P. \& Van der Meer, T. H.

Mean temperature profile at the entrance of a thermoacoustic stacked heat exchanger

9th PAMIR International Conference, CNRS - IPUL, 2014, 407-411

Bühler, S.; Wilcox, D.; Oosterhuis, J. P. \& Van der Meer, T. H.

Mean temperature profile at the entrance of a thermoacoustic stacked screen heat exchanger

Magnetohydrodynamics, 2015, 51, accepted

Oosterhuis, J. P.; Bühler, S.; Wilcox, D. \& Van der Meer, T. H.

CFD as a Design Tool for a Concentric Heat Exchanger

9th International Conference on Heat Transfer, Fluid Mechanics and Thermodynamics, 2012, 110-115

Oosterhuis, J. P.; Bühler, S.; Wilcox, D. \& Van der Meer, T. H.

Design of a concentric heat exchanger using computational fluid dynamics as a design tool

Computational Thermal Sciences, 2013, 5, 165-176

Oosterhuis, J. P.; Bühler, S.; Wilcox, D. \& Van der Meer, T. H.

Computational fluid dynamics simulation of Rayleigh streaming in a vibrating resonator

Proceedings of Meetings on Acoustics, Vol. 19, ASA, 2013

Oosterhuis, J. P.; Bühler, S.; Wilcox, D. \& Van der Meer, T. H.

Computational fluid dynamics analysis of the oscillatory flow in a jet pump: the influence of taper angle

9th PAMIR International Conference, CNRS - IPUL, 2014, 391-395 
Oosterhuis, J. P.; Bühler, S.; Wilcox, D. \& Van der Meer, T. H.

A numerical investigation on the vortex formation and flow separation of the oscillatory flow in jet pumps

Journal of the Acoustical Society of America, 2015, 137, 1722-1731

Oosterhuis, J. P.; Bühler, S.; Wilcox, D. \& Van der Meer, T. H.

Jet pumps for thermoacoustic applications: design guidelines based on a numerical parameter

Journal of the Acoustical Society of America, 2015, submitted 



\section{Acknowledgments}

What a great feeling when you can submit your $\mathrm{PhD}$ thesis after four years of work and see all this work finalized. With this great feeling in mind I want to thank all the people that contributed to this successful time, because without you this would not have been possible.

Four years ago I got in touch with thermoacoustics for the first time and from the very first moment on you, Doug, made me enthusiastic about this topic. You accompanied me during the four years with helpful discussions and reminded me of my initial enthusiasm when it was necessary. Thanks for sharing your precious time and experience with us.

Als mijn dagelijkse begeleider wil ik jou, Theo, ook bedanken voor de maandagochtend discussies en dat jouw deur altijd open stond voor vragen. In die vier jaar heb ik van jou geleerd me om sommige dingen minder druk te maken, omdat uiteindelijk toch alles goed komt. Deze rust neem ik graag mee.

Tegenwoordig is de financiering een van de belangrijkste, zo niet de belangrijkste uitdaging in het onderzoek. Daarom wil ik Agentschap NL bedanken voor het financieren van dit project, in samenwerking met Bosch en Aster Thermoacoustics. Anne, Ysbrand, Sjoerd, Sungbae, David en Kees, ik vond het spannend jullie visies op thermoakoestiek en het project te ervaren.

Dan wil ik natuurlijk ook jou, Joris, van harte bedanken, dat ik dit hier nu in het Nederlands kan schrijven is grotendeels ook dankzij jou. Je bleef maar Nederlands tegen mij praten, ook tijdens onze vele discussies, ongeacht hoe moeilijk het te begrijpen was. Ook op onze spannende reizen in het buitenland ging het in het Nederlands door. Verder heb jij samen met Joost mij de Nederlandse cultuur laten beleven en mij de betekenis van het Nederlandse woord "gezellig" geleerd, tijdens kantooruren maar ook daar buiten. Ook motiveren konden jullie mij goed, als ik weer het liefst met alles gestopt was.

Rob en Bart jullie hebben mij in het kader van jullie masteropdracht ondersteund, en mij met nieuwe ideeën gestimuleerd. Graag denk ik terug aan onze discussies, vaak 's avonds laat op de UT als iedereen al weg was en aan de tijd buiten de UT die we samen hadden. Daan, Citra, Rully and Michael, thanks all of you for your commitment to our thermoacoustic project and for bringing it a step further.

I want to thank also my roommates Anton, Joost, Joris, Laia and Pawel for the great atmosphere in our room and for the numerous discussions we had that distracted us from working. This was a great time working next to you! 
Sally, when something had to be organized you were always of great help, thanks. And of course I want to thank also all the other members of the thermal engineering group, for all the great activities and time we had together.

Maar gezelligheid en steun is ook buiten het werk heel erg belangrijk om zo'n groot project af te ronden. Hockey was perfect om afstand van het werk te krijgen. Ik had vier mooie jaren met jullie, waar wij fanatiek gehockeyd hebben. Jullie hebben van het hockeyen een heel belangrijk deel van mijn tijd in Enschede gemaakt. Graag kijk ik terug naar onze uitwedstrijden met lunch of ontbijt in de trein en de vrolijke terugreizen, de leuke gesprekken op de toren en dat jullie mij Nederlands geleerd hebben. Rotzooi.

Lars, Anne, Finn und Alena ihr wart meine kleine Familie in Enschede. Für die Kraft die ich aus der gemeinsamen Zeit schöpfen konnte, möchte ich mich ganz herzlich bedanken!

Mama, Papa und Adri, von euch habe ich diesen Drang immer wieder neues zu erleben und ihr habt mir bei allem was ich gemacht habe immer den Rücken frei gehalten. Nur so konnte ich es bis hier her schaffen. Dafür von tiefem Herzen vielen Dank!

Even though this chapter of my life ends with this book, I will keep all of you in my heart! Thanks for the great time. 



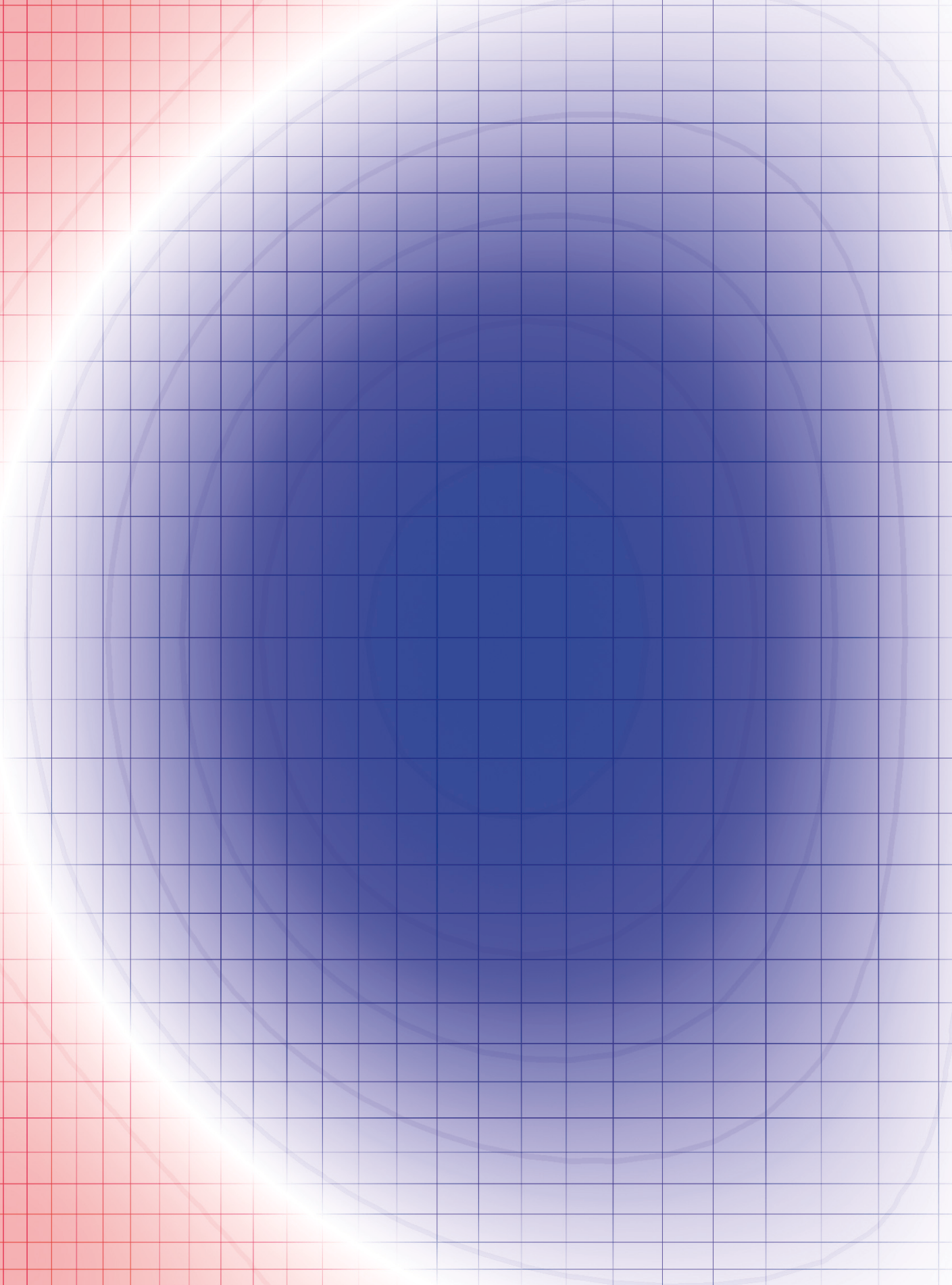


Propositions accompanying the dissertation

\title{
TOWARDS NUMERICAL SIMULATION OF COMPONENTS OF THERMOACOUSTIC DEVICES WITH COMMERCIAL CFD SOFTWARE
}

\author{
Implementation of impedance boundary conditions \\ and application to four different studies
}

1. Developing an accurate turbulence model for CFD simulation of purely oscillating acoustic flow is one of the most important steps for accurate modelling of practical thermoacoustic devices.

2. Designing thermoacoustic devices only with full time domain CFD is out of reach in the near future.

3. CFD is all about the right boundary conditions.

4. The quicker the feedback loops from experiments, the steeper the learning curve can be.

5. Having a second $\mathrm{PhD}$ on the same topic increases the scientific output considerably over proportionally.

6. Too much importance is attributed to the number of publications in the evaluation of the quality and progress of scientific work.

7. Paper writing is an outdated way of scientific reporting.

8. At the beginning of a new $\mathrm{PhD}$ project it can be more successful to take action right away, than trying to carefully weight all possibilities.

9. De uitspraak: "Karakter zonder kennis leidt vaker tot succes dan kennis zonder karakter" van Frank de Boer is ook van toepassing op het uitvoeren van wetenschappelijk onderzoek.

These propositions are considered opposable and defendable and as such have been approved by the promotor Prof. dr. ir. T.H. van der Meer. 